\title{
Platelet Toll-like Receptors in Healthy and Acute Myocardial Infarction Subjects.
}

\author{
By
}

Kathryn Elizabeth Hally

A thesis submitted to the Victoria University of Wellington in fulfilment of the requirements for the degree of Doctor of Philosophy

Victoria University of Wellington 


\section{Abstract.}

Platelet activation is pathological in acute myocardial infarction (AMI). Despite treatment with dual anti-platelet therapy (DAPT), platelet activation can continue to occur post-AMI and has been linked to an increased risk of recurrent cardiovascular events. Toll-like receptors (TLRs) are important innate immune receptors, and platelets are known to express a subset of TLRs. The functional significance of these platelet-TLR pathways in AMI has not been fully examined but may contribute to persistent post-AMI platelet activation. Platelet-TLR expression, TLR-mediated platelet activation and the platelet effect on leukocyte responses to TLR stimulation were examined in this thesis.

Platelet-TLR expression and TLR-mediated platelet activation was examined for a subset of these receptors (TLR1, 2, 4, 6 and 9) in healthy subjects and in AMI subjects on DAPT. We observed an increase in platelet expression of TLR1, 4 and 9 in AMI platelets compared to healthy subjects. Further investigation into platelet-TLR9 expression showed an increase in expression upon platelet activation in healthy, but not AMI, subjects. We observed direct, dose-dependent platelet activation in response to Pam3CSK4 (TLR2/1 agonist) and ODN2009 (TLR9 agonist) in healthy subjects and in AMI on DAPT. For both cohorts, platelets were also directly activated by a high dose of LPS (TLR4 agonist) but were not directly activated by FSL-1 (TLR6 agonist). These results demonstrate that some (TLR1, 2, 4 and 9), but not other (TLR6), platelet-TLR pathways can cause platelet activation in AMI despite treatment with potent anti-platelet therapy.

For the results described above, we were unable to assess TLR-mediated platelet activation in the absence of anti-platelet therapy in AMI subjects as these drugs are administered before or immediately upon presentation to hospital. It was therefore not possible to exclude the possibility that DAPT was providing a degree of inhibition of platelet activation in AMI patients. To address this, we determined the extent to which aspirin monotherapy or DAPT could inhibit platelet 
activation in response to TLR2/1, TLR4 and TLR9 stimulation in a cross-over study in healthy subjects. We demonstrated that DAPT only modestly inhibited, and aspirin monotherapy did not inhibit, platelet activation in response to all TLR agonists tested and platelets still became potently activated despite treatment with anti-platelet agents. These platelet-TLRs represent intact ontreatment platelet activation pathways.

Lastly, we determined the extent to which platelets modulate leukocyte responses to TLR2/1, TLR2/6 and TLR4 stimulation. Platelets were able to reduce neutrophil responses to TLR stimulation, and modulated PBMC cytokine and chemokine production in a complex manner following stimulation with LPS and FSL-1. The presence of platelets did not change cytokine/chemokine production in response to Pam3CSK4, demonstrating a TLR agonist-specific manner of platelet modulation. We further investigated the effect of platelets on neutrophil responses to TLR stimulation. With platelets, neutrophil activation was attenuated, and phagocytic activity was increased in unstimulated cultures and in response to various doses of Pam3CSK4 and FSL-1. Neutrophil elastase secretion was attenuated in unstimulated cultures and in response to low-dose stimulation with all three TLR agonists. We show that platelets can both augment and attenuate various markers of neutrophil function.

Together, this work indicates that platelets express functional TLR pathways that can differentially regulate a number of thrombotic and inflammatory responses in healthy subjects and in subjects with AMI. 


\section{Acknowledgements.}

First and foremost, to my supervisors A. Prof. Peter Larsen, Dr. Scott Harding and Prof. Anne La Flamme. Thank you for giving me your invaluable support, knowledge and encouragement throughout this process. For all that you have taught me, I am extremely grateful.

To all of the staff at the Department of Cardiology, Wellington Hospital, for being welcoming and patient. Thank you for helping this scientist navigate the world of translational research. To all of the volunteers that have participated in these studies, thank you for your co-operation and your interest, and know that this work was not possible without your contribution.

To all of the kind and brilliant people that I have had the good fortune to meet along the way, thank you for your constant guidance and friendship. I feel very privileged to have worked with each and every one of you. A special thank you to Aimee and Ana, who have been here from the start, you two are such stars.

To my wonderful family, for the support and love you have shown me, always. To my Mum and Dad, for your endless faith in me and for always reminding me how much I'm loved. To my Grandad, for giving me your strength and resilience. To my brother, for your unwavering pride in me and my work. And finally, to Charlie, for always encouraging me to reach further and achieve more than I ever thought I could. You are my rock. 


\section{Table of contents.}

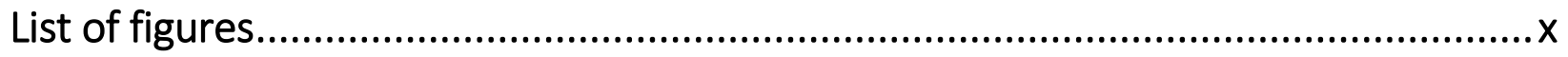

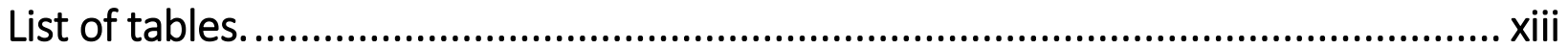

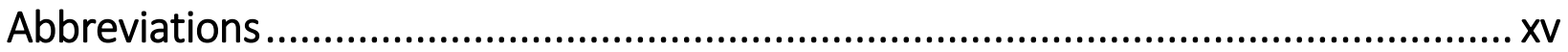

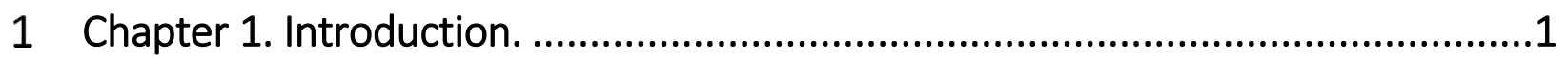

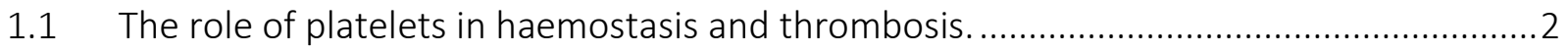

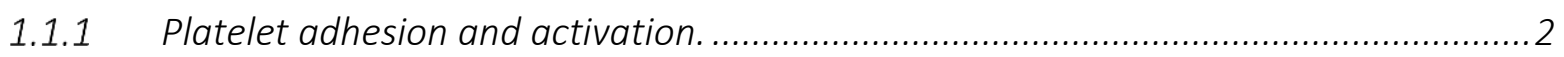

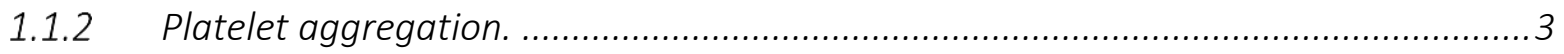

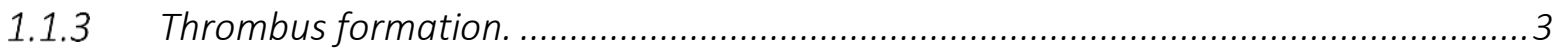

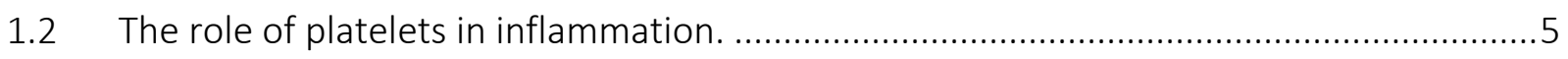

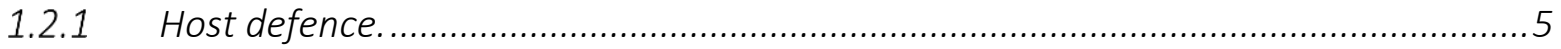

1.2.2 Platelets can modulate leukocyte inflammatory responses....................................6

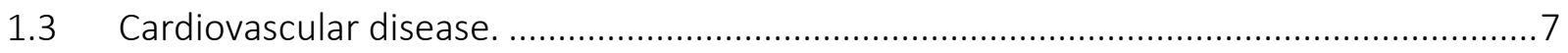

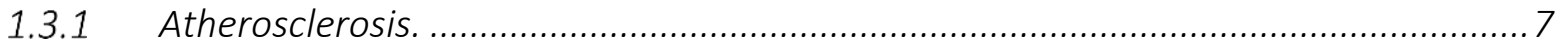

1.3.2 Acute coronary syndromes (ACS): definitions...................................................11

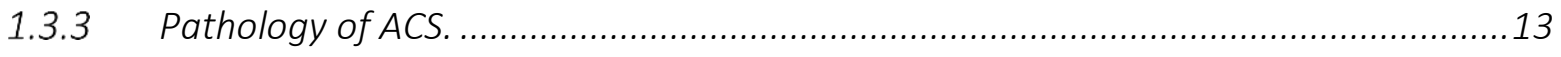

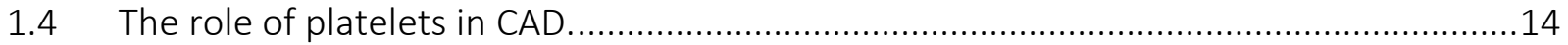

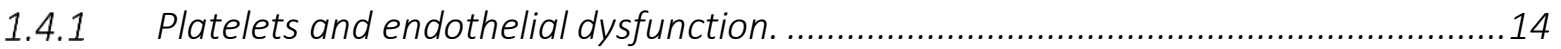

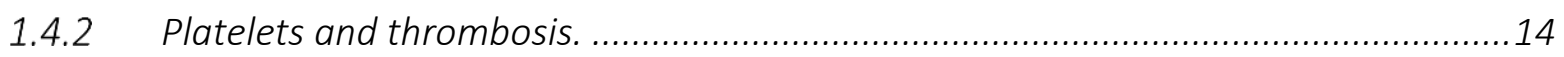

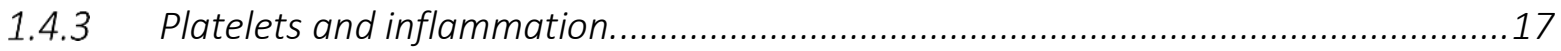

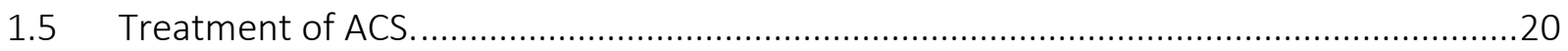

1.5 .1 Aspirin

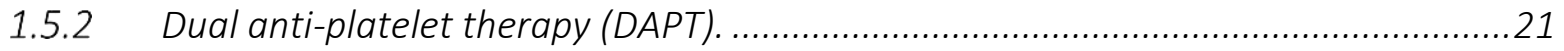

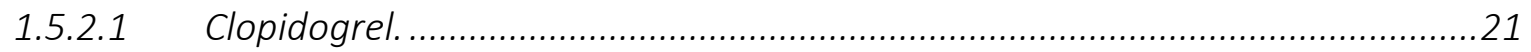

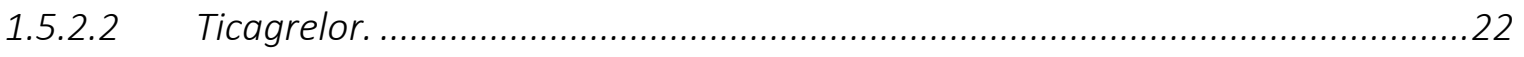

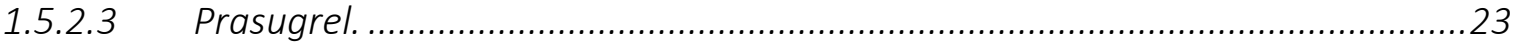

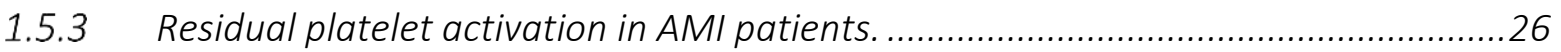

1.6 Alternative pathways of platelet activation: platelets and infection...............................27

1.7 Toll-like receptors (TLRs): the first effectors of the response to infection.......................28 


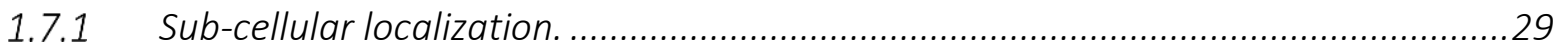

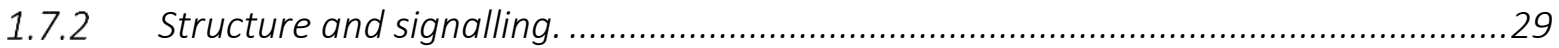

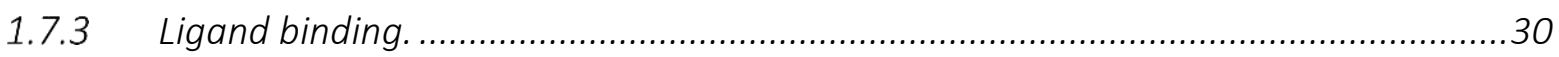

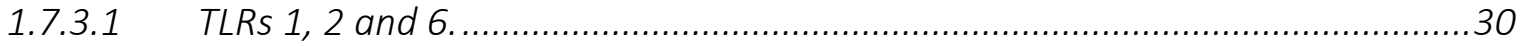

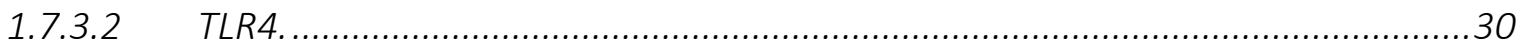

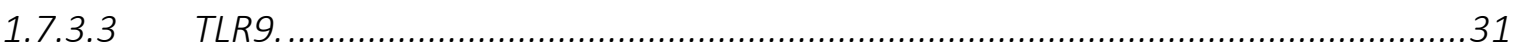

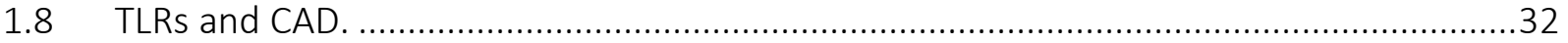

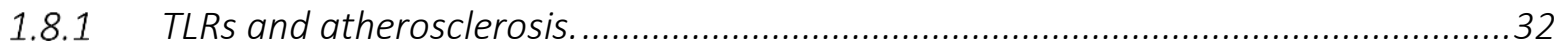

1.8.2 TLRs and ACS: the role of TLRs in myocardial ischaemia/reperfusion injury.............37

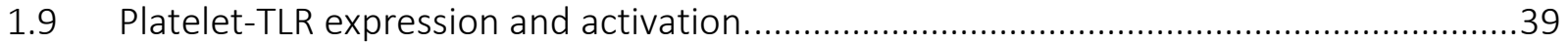

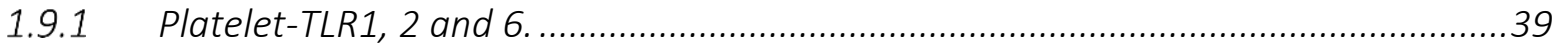

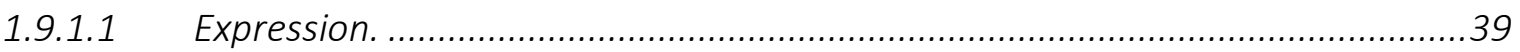

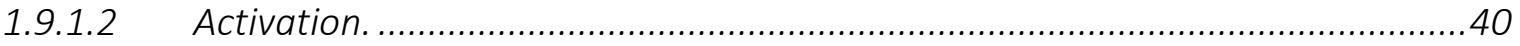

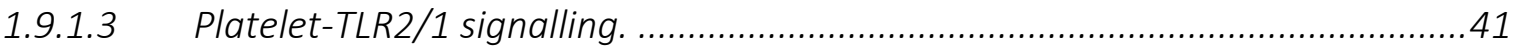

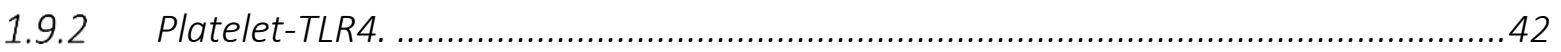

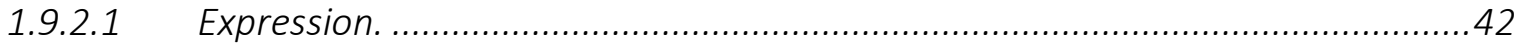

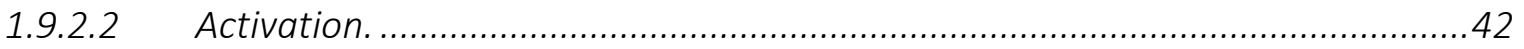

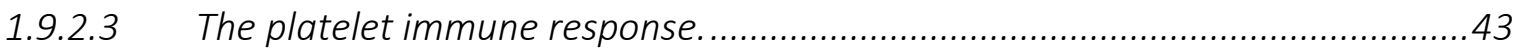

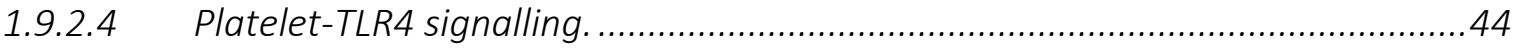

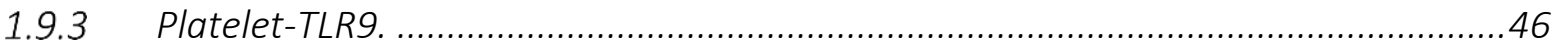

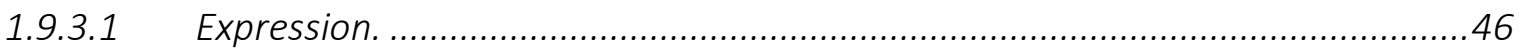

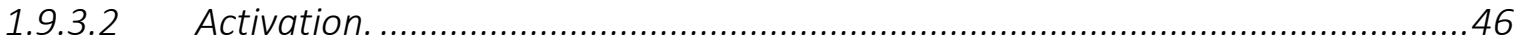

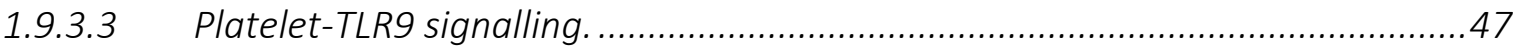

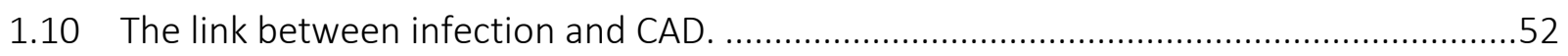

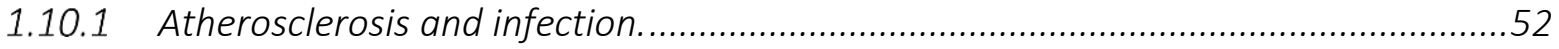

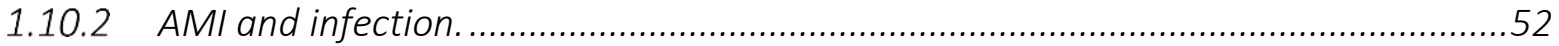

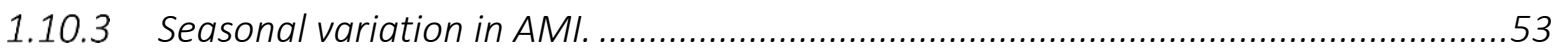

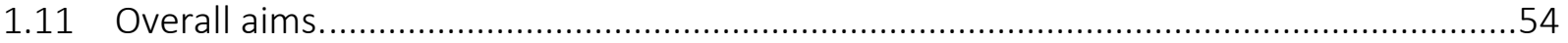

\section{Chapter 2. TLR9 expression and ODN2006-mediated platelet activation in AMI}

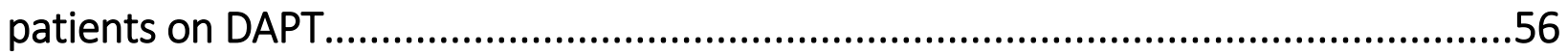

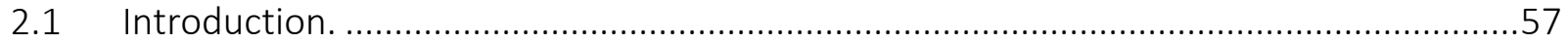




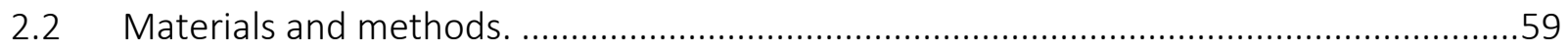

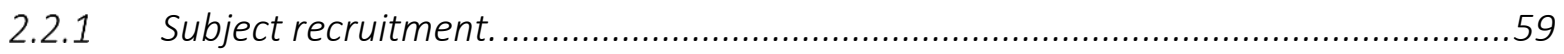

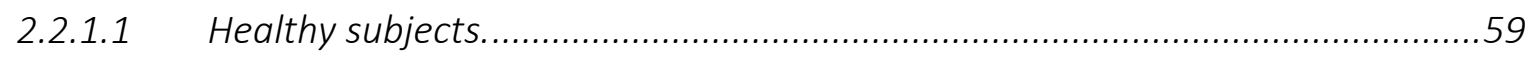

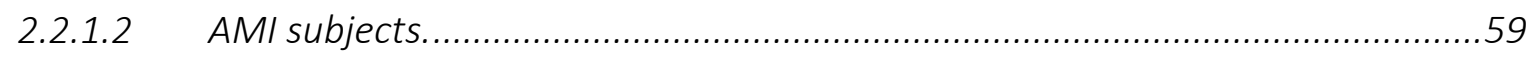

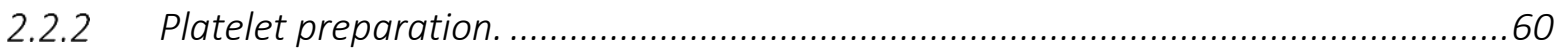

2.2.3 Flow cytometric analysis of TLR9 expression.........................................................60

2.2.4 Western blot analysis of TLR9 expression.......................................................65

2.2.5 Flow cytometric detection of ODN2006-mediated platelet activation.....................66

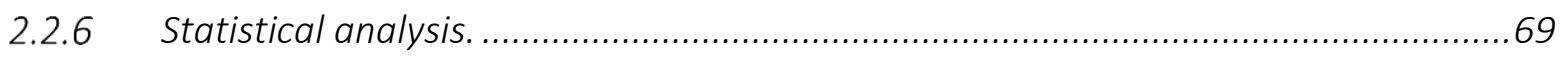

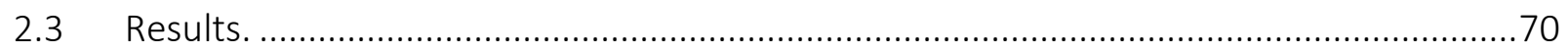

2.3.1 TLR9 expression in healthy and AMI subjects. .................................................. 70

2.3.2 Dose response curves for ODN2006-mediated platelet activation........................... 72

2.3.3 Demographics for ODN-mediated platelet activation........................................73

2.3.4 Platelet activation in healthy subjects following ODN2006 agonism......................75

2.3.5 Platelet activation in AMI subjects following ODN2006 agonism. ..........................77

2.3.6 Comparison of PRP and WB responses to ODN2006 ...........................................79

2.3.7 Comparison of ODN2006-mediated platelet activation across cohorts...................80

2.3.8 A differential platelet response to 2.5 $\mu \mathrm{M}$ TRAP was seen between cohorts............81

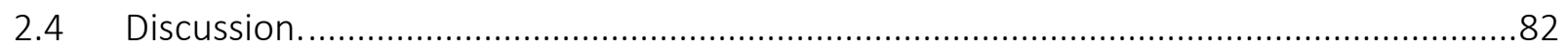

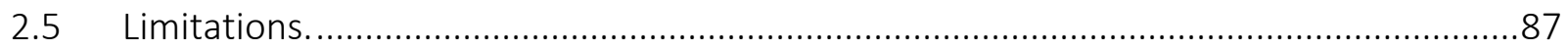

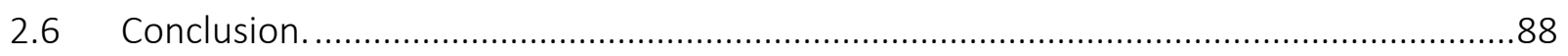

\section{Chapter 3. Platelet Toll-like receptor 1, 2, 4 and 6 expression and TLR-mediated}

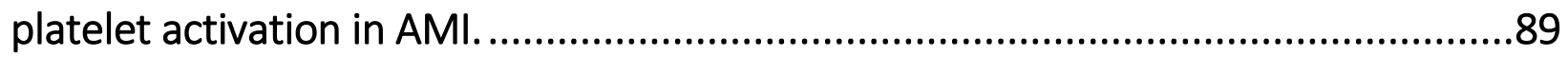

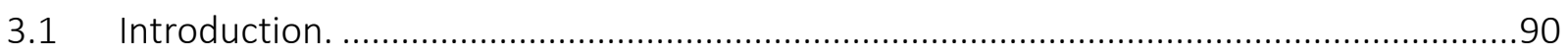

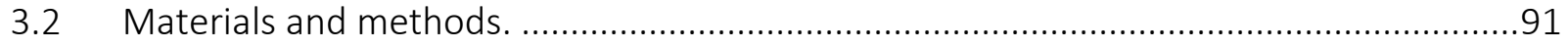

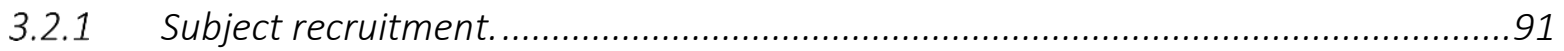

3.2.2 Whole platelet lysate preparation for western blotting ........................................91

3.2.3 Platelet-TLR expression by western blotting..................................................92

3.2.4 TLR-mediated platelet activation by flow cytometry............................................94

3.2.5 TLR-mediated platelet-monocyte aggregation (PMAgg) by flow cytometry. ..........98

3.2.6 TLR-mediated monocyte activation by flow cytometry.....................................100 


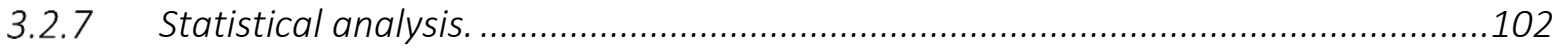

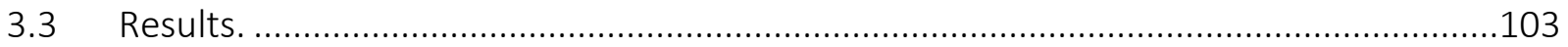

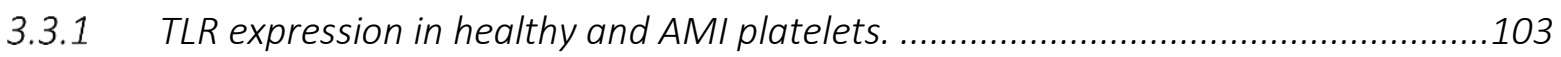

3.3.2 TLR-mediated platelet activation in healthy and AMI platelets.............................106

3.3.2.1 Platelet activation in response to LPS stimulation........................................106

3.3.2.2 Platelet activation in response to Pam3CSK4 ...........................................113

3.3.2.3 Platelet activation in response to FSL-1 stimulation...................................115

3.3.3 Platelet-monocyte aggregation (PMAgg) in response to TLR agonists..................115

3.3.4 Dose-dependent monocyte activation in response to TLR agonism.....................118

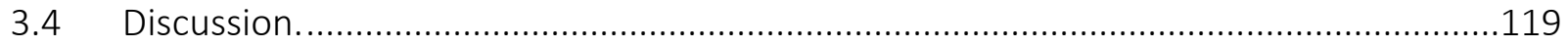

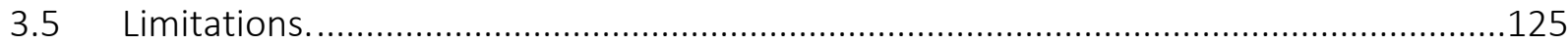

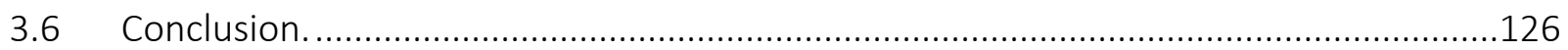

\section{Chapter 4. The effects of aspirin and ticagrelor on TLR-mediated platelet} activation: results of a randomised, cross-over trial...........................................127

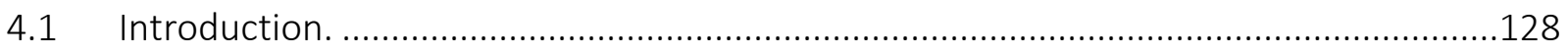

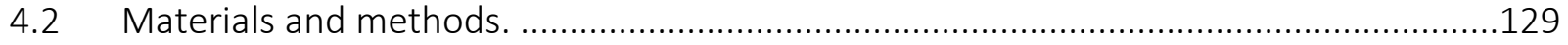

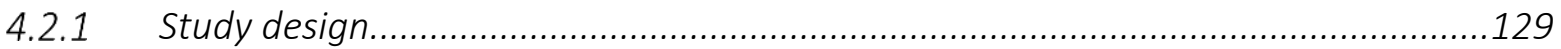

4.2.2 Blood drawing and platelet aggregation assay.............................................131

4.2.3 Flow cytometric detection of TLR-mediated platelet activation..........................131

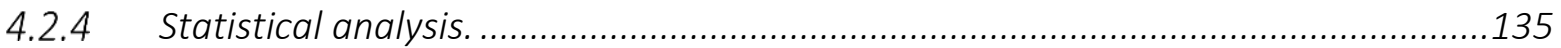

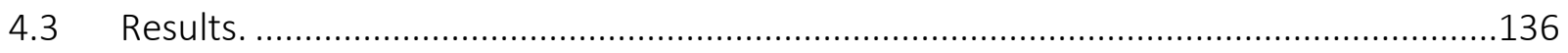

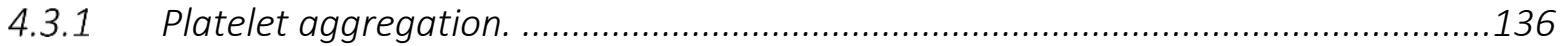

4.3.2 Period effect and treatment-by-period interaction analysis. ................................137

4.3.3 TLR-mediated platelet activation at baseline. .....................................................139

4.3.4 The effect of anti-platelet therapy on TLR-mediated platelet activation...............142

4.3.4.1 TLR-mediated platelet activation following aspirin therapy. .........................142

4.3.4.2 TLR-mediated platelet activation following DAPT ........................................144

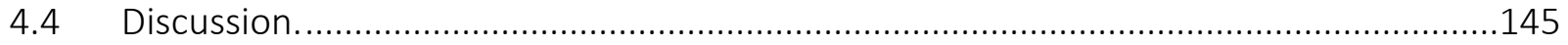

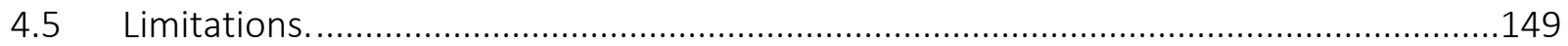

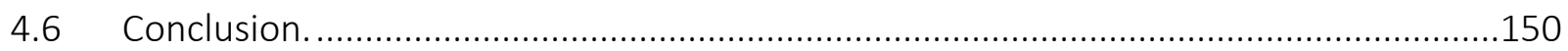




\section{Chapter 5. Platelets modulate leukocyte function in response to TLR stimulation. 151}

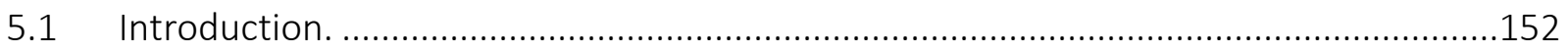

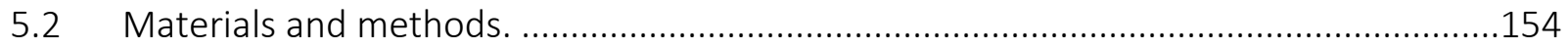

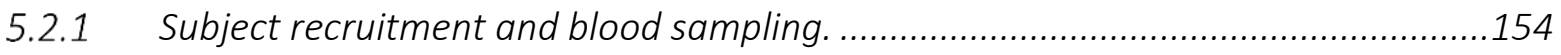

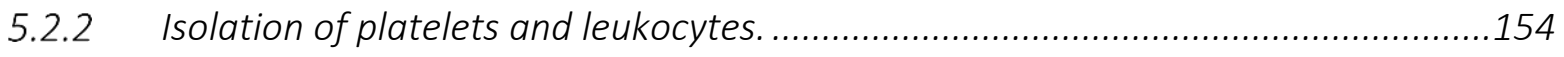

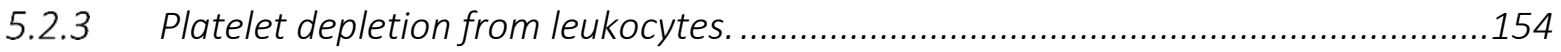

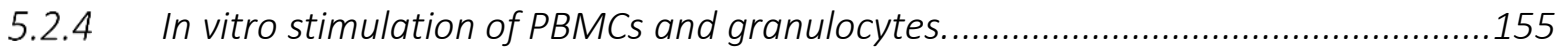

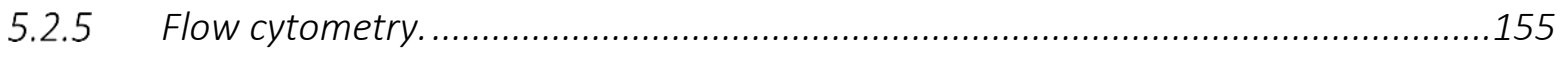

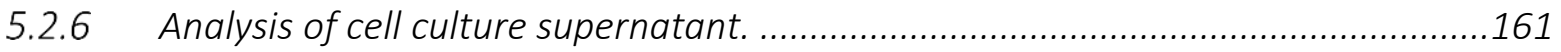

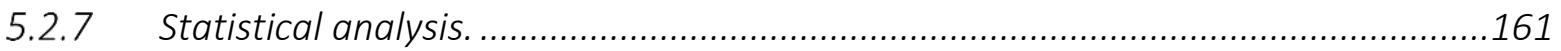

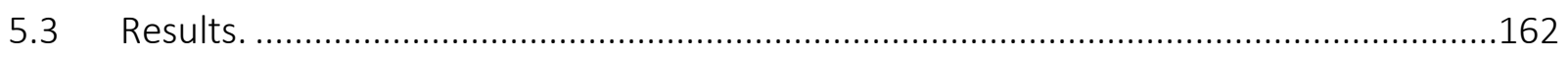

5.3.1 Platelets reduce neutrophil CD66b expression in response to TLR stimulation.......162

5.3.2 Platelets modestly reduce monocyte HLA-DR expression following low-dose LPS. 166

5.3.3 The addition of platelets modulates CD8+T cell, but not CD4+T cell, CD38 expression in response to TLR stimulation. .....................................................................168

5.3.4 Platelet co-culture reduces elastase secretion following low-dose TLR agonism...170

5.3.5 Platelets differentially modulate IL-6 production in response to LPS and FSL-1....173

5.3.6 Platelets reduce TNF- $\alpha$ production in response to LPS and FSL-1.........................176

5.3.7 Platelets increase IL-10 production by PBMCs in response to LPS and FSL-1.........178

5.3.8 Platelets differentially modulate MIP-16 production in response to some TLR agonists. 180

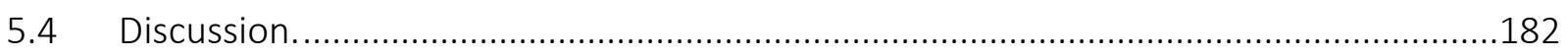

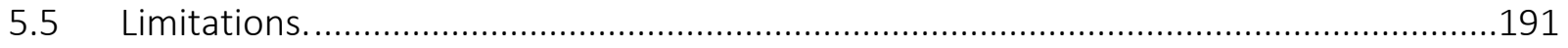

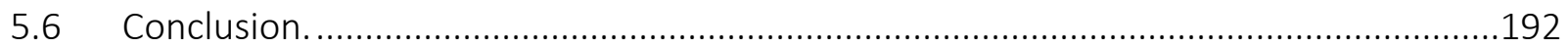

6 Chapter 6. Platelets modulate multiple markers of neutrophil function in

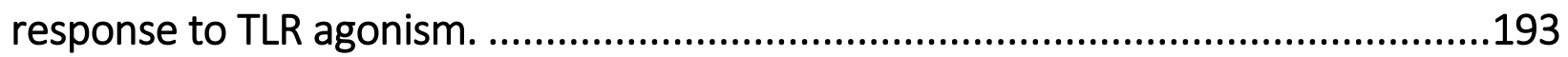

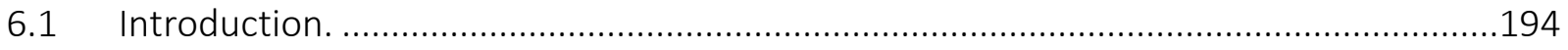

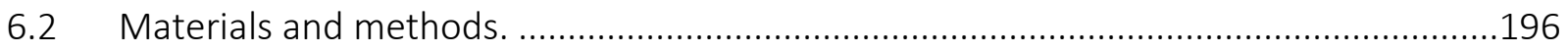

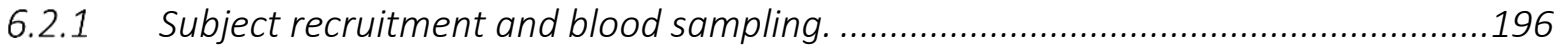

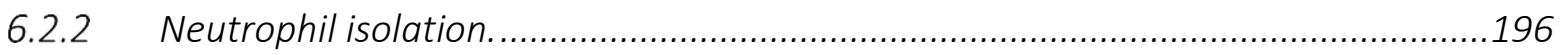




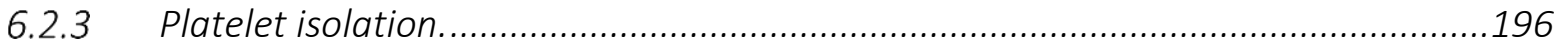

6.2.4 In vitro TLR stimulation and assessment of neutrophil activation markers. ..........197

6.2.5 In vitro TLR stimulation and assessment of neutrophil phagocytosis...................201

6.2.6 Analysis of cell culture supernatant. ............................................................203

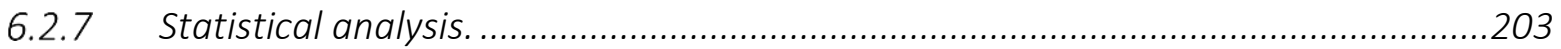

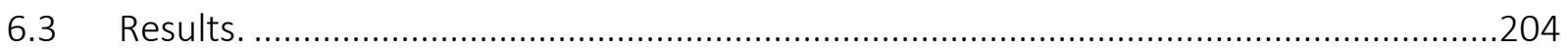

6.3.1 Platelets reduce CD66b expression in response to Pam3CSK4 and FSL-1............204

6.3.2 Platelets modulate neutrophil CD11b expression following stimulation with

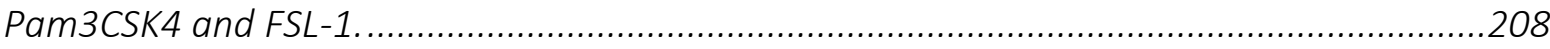

6.3.3 Platelets modulate neutrophil CD62L expression in unstimulated cultures and in response to some TLR stimulation conditions.

6.3.4 With platelets, neutrophil elastase production was attenuated in unstimulated cultures and in response to low-dose TLR stimulation.

6.3.5 Neutrophil IL-8 production is decreased in the presence of platelets following lowdose LPS stimulation.

6.3.6 The addition of platelets modulates neutrophil phagocytosis in unstimulated cultures and in response to some TLR stimulation conditions.

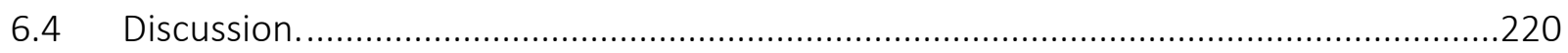

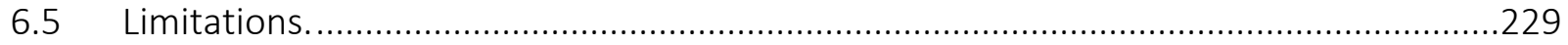

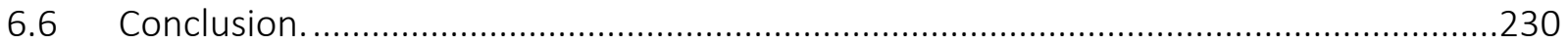

7 Chapter 7. Summary and future directions................................................231

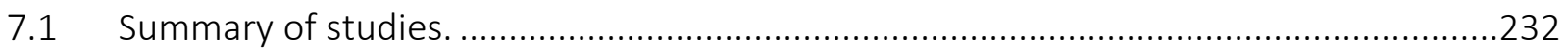

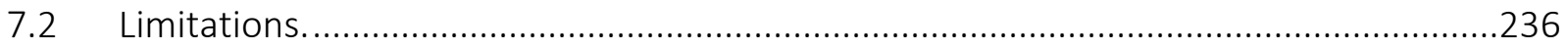

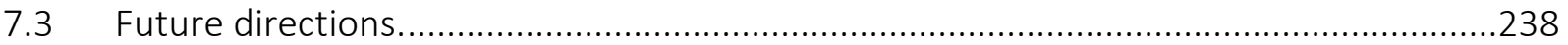

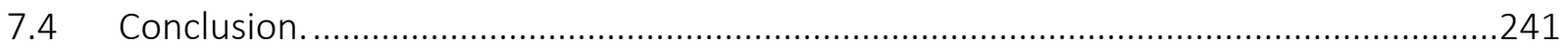

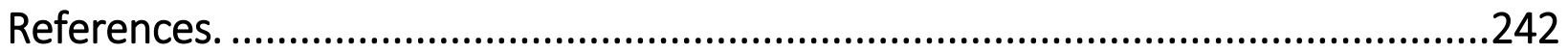

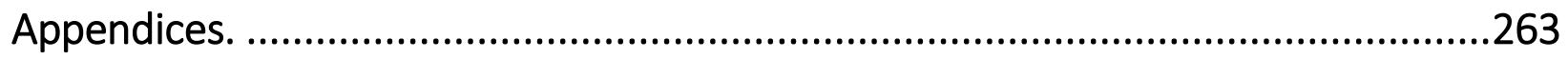

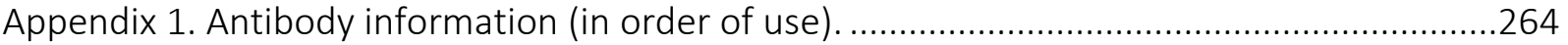

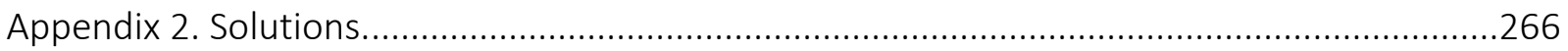




\section{List of figures}

Figure 1.1 The development of the atherosclerotic plaque. 10

Figure 1.2 Activated platelets contribute to the formation of coronary thrombi during atherosclerotic plaque rupture and ACS. 16

Figure 1.3 Activated platelets contribute to atherosclerosis and ACS. .19

Figure 2.1 The platelet and monocyte populations were identified by flow cytometry. 63

Figure 2.2 TLR9 expression in healthy and AMI platelets was assessed by flow cytometry. 64 Figure 2.3 Identification of the platelet population and of the platelet activation markers, CD62p and CD63. 68

Figure 2.4 TLR9 expression increases in AMI platelets and upon platelet activation, compared to resting healthy platelets. 71

Figure 2.5 Dose-response curves for TRAP and ODN2006. 72

Figure 2.6 Incubation with ODN2006 in WB and PRP induced significant platelet activation in healthy subjects. .76

Figure 2.7 Incubation with ODN2006 in WB and PRP induced significant platelet activation in AMI subjects. 78

Figure 2.8 Platelet activation in response to ODN2006 is greater in WB than in PRP.... 79

Figure 2.9 Platelet activation in response to ODN2006 is similar across cohorts. 80 Figure 2.10 Platelet activation in response to $2.5 \mu \mathrm{M}$ TRAP in WB is greater in the healthy cohort.

Figure 3.1 Representative examples of TLR1, 2, 4 and 6 expression in resting platelet lysates are shown for healthy $(n=2)$ and AMI ( $n=2)$ subjects. .93

Figure 3.2 Platelets were identified by flow cytometry.........................................................96

Figure 3.3 Representative plots of platelet activation markers. .................................................97

Figure 3.4 Platelet monocyte aggregation was measured by flow cytometry. ...........................99

Figure 3.5 Representative plots of monocyte CD63 expression 101 Figure 3.6 AMI platelets express higher levels of TLR1 and TLR4, but not TLR2 or TLR6, compared to healthy platelets. .105 
Figure 3.7 TLR1, 4 and 6 expression shows a bimodal distribution in the AMI cohort. 105

Figure 3.8 Platelets upregulate CD62p in response to TLR agonism in PRP. .107

Figure 3.9 Platelets express CD62p in response to TLR agonism in WB. 108

Figure 3.10 PAC1 is upregulated on platelets following incubation with TLR agonists in PRP....110

Figure 3.11 Variable PAC1 expression is seen in response to TLR agonism in WB..... .111

Figure 3.12 Platelets are dose-dependently activated in response to Pam3CSK4. .114

Figure 3.13 Increased PMAgg occurs following TLR agonism in the healthy and AMI cohort.....116 Figure 3.14 A dose-dependent increase in monocyte activation was observed in response to TLR agonism. .118

Figure 4.1 Study cross-over protocol. .130

Figure 4.2 Platelets were identified by flow cytometry. 133

Figure 4.3 Representative plots of activation markers. .134

Figure 4.4 Platelet aggregation testing confirmed a significant inhibitory effect of the antiplatelet drug regimens on each of the targeted platelet activation pathways. .136

Figure 4.5 Platelet PAC1 expression in response to TLR stimulation is modestly attenuated following DAPT, but not aspirin, administration. .140

Figure 4.6 CD62p expression is modestly modulated in response to TLR stimulation following aspirin and DAPT administration. .141

Figure 5.1 Live monocytes and lymphocytes were identified from PBMCs. .157

Figure 5.2 CD64+ monocytes and CD4+ and CD8+ T cells were identified from PBMCs. .158

Figure 5.3 Live CD16+ neutrophils were identified from granulocytes. .159

Figure 5.4 Representative plots of leukocyte activation markers. 160

Figure 5.5 In response to TLR stimulation, platelets modulate expression of neutrophil CD66b expression. .164

Figure 5.6 Platelets modestly modulate monocyte HLA-DR expression following low-dose LPS stimulation. .167

Figure 5.7 Platelets modulate expression of CD8+, but not CD4+, T cell expression of CD38 following TLR stimulation. 169 
Figure 5.8 Platelets attenuated granulocyte elastase secretion in response to low-dose TLR agonism.

Figure 5.9 Distinct patterns of IL-6 production were seen following TLR stimulation and platelet co-culture.

Figure 5.10 TNF- $\alpha$ levels were attenuated with platelet co-culture following stimulation with LPS and FSL-1.

Figure 5.11 IL-10 production was increased following stimulation with high-dose LPS and with FSL-1 and platelet co-culture.

Figure 5.12 Platelets modulate MIP-1 $\beta$ production were seen following stimulation with low-

dose LPS and with FSL-1. 181

Figure 6.1 CD16+ neutrophils were identified. .199

Figure 6.2 Representative plots of neutrophil activation markers. 200

Figure 6.3 Representative plot of neutrophil phagocytosis. 202

Figure 6.4 Platelets modulate expression of neutrophil CD66b expression in response to Pam3CSK4 and FSL-1. 207

Figure 6.5 CD11b expression is altered by platelets in response to Pam3CSK4 and FSL-1.........209 Figure 6.6 CD62L expression is altered by platelets in unstimulated cultures and in response to some TLR stimuli. .211

Figure 6.7 Platelets attenuate elastase secretion in unstimulated cultures and in response to low-

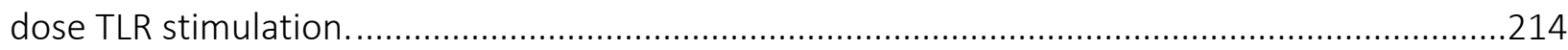
Figure 6.8 Platelets modulate IL-8 secretion only in response to low-dose LPS. .216 Figure 6.9 Neutrophil phagocytosis is altered by platelets in unstimulated cultures and following low-dose Pam3CSK4. 219 


\section{List of tables.}

Table 1.1 The spectrum of acute coronary syndromes. ........................................................12

Table 1.2 Guidelines for the treatment of ACS with oral anti-platelet agents. ...........................24

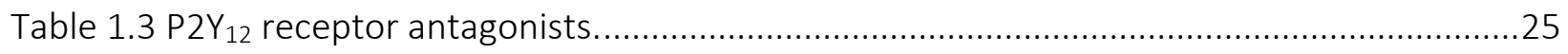

Table 1.4 Evidence for involvement of TLRs in atherosclerosis................................................34

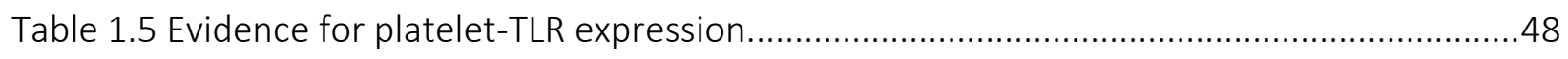

Table 1.6 Evidence for TLR-mediated platelet activation. ..........................................................50

Table 2.1 Panel design for TLR9 expression in platelets and monocytes. .....................................62

Table 2.2 Panel design for ODN2006-mediated platelet activation............................................67

Table 2.3 Demographics and clinical characteristics of the AMI and healthy cohort. ..................74

Table 3.1 Panel design for assessing platelet activation in response to TLR agonism..................95

Table 3.2 Panel design for assessing platelet-monocyte aggregation following TLR agonism.......98

Table 3.3 Panel design for assessing monocyte activation in response to TLR agonism.............100

Table 3.4 Demographics and clinical characteristics of each cohort for investigating TLR

expression and TLR-mediated platelet activation. .................................................................. 104

Table 3.5 Incubation with a range of TLR agonists induces variable CD62p-positivity in the healthy and $\mathrm{AMI}$ cohorts.

Table 3.6 Incubation with a range of TLR agonists induces platelet PAC1-positivity in the healthy and $\mathrm{AMI}$ cohorts.

Table 3.7 Increased PMAgg occurs following incubation with a range of TLR agonists in the healthy and AMI cohorts.

Table 4.1 Panel design for assessing platelet activation in response to TLR agonism.

Table 4.2 Group A received aspirin monotherapy (period 1) followed by DAPT (aspirin and ticagrelor) (period 2), $\mathrm{n}=5$......

Table 4.3 Group B received aspirin and ticagrelor (period 1) followed by aspirin monotherapy (period 2), $\mathrm{n}=5$.

Table 4.4 Absolute changes in the percentage of the platelet population that was PAC1+ and CD62p+ following aspirin monotherapy and DAPT. 
Table 5.1 Panel design for assessing leukocyte activation in response to TLR agonism. .156

Table 5.2 Baseline (- platelets) geometric MFI of leukocyte activation markers in response to TLR agonism, compared to unstimulated cultures. .163

Table 5.3 Relative change in leukocyte activation markers in leukocyte-platelet co-culture (+ platelets) and following TLR stimulation. .165 Table 5.4 Relative change in elastase secretion $(\mathrm{ng} / \mathrm{mL})$ in granulocyte-platelet co-culture (+ platelets) and following TLR stimulation.

Table 5.5 Relative change in PBMC cytokine and chemokine production in PBMC-platelet coculture (+ platelets) and following TLR stimulation.

Table 5.6 Summary of the effect of platelets on the markers of leukocyte function assessed in this study. 183

Table 6.1 Panel design for assessing leukocyte activation in response to TLR agonism. 198 Table 6.2 Baseline (- platelets) geometric MFI of neutrophil activation markers in response to TLR agonism, compared to unstimulated cultures. .205

Table 6.3 Relative change in neutrophil activation markers in neutrophil-platelet co-culture (+ platelets) and following TLR stimulation. 206

Table 6.4 Baseline (- platelets) levels of neutrophil secretory products in response to TLR agonism, compared to unstimulated cultures.

Table 6.5 Relative change in neutrophil secretory products in neutrophil-platelet co-culture (+ platelets) and following TLR stimulation. 213 Table 6.6 Baseline (- platelets) neutrophil phagocytosis, as measured by the percentage of FITCpositive neutrophils (\%FITC+), in response to TLR agonism compared to unstimulated cultures.

Table 6.7 Relative change in phagocytosis (\%FITC+ neutrophils) in neutrophil-platelet co-culture (+ platelets) and following TLR stimulation. .218 Table 6.8 Summary of the effect of platelets on the markers of neutrophil function assessed in this study. 


\section{Abbreviations}

\begin{tabular}{|c|c|}
\hline ACS & Acute coronary syndrome \\
\hline ADP & Adenosine diphosphate \\
\hline $\mathrm{AHA}$ & American Heart Association \\
\hline AMI & Acute myocardial infarction \\
\hline CABG & Coronary artery bypass graft \\
\hline CAD & Coronary artery disease \\
\hline CAPs & 2-( $\omega$-Carboxyalkyl) pyrrole protein adducts \\
\hline CD40L & CD40 ligand \\
\hline CK-MB & Creatine kinase-myocardial band \\
\hline CLARITY & The Clopidogrel as Adjunctive Reperfusion Therapy trial \\
\hline $\operatorname{cox} 1$ & Cyclooxygenase-1 \\
\hline CpG & Cytidine-guanosine \\
\hline CREDO & The Clopidogrel for the Reduction of Events During Observation trial \\
\hline CURE & The Clopidogrel in Unstable Angina to Prevent Recurrent Events trial \\
\hline CVD & Cardiovascular disease \\
\hline CYP & Cytochrome P \\
\hline DAMPs & Danger associated molecular patterns \\
\hline DAPT & Dual anti-platelet therapy \\
\hline ECG & Electrocardiogram \\
\hline ESC & European Society of Cardiology \\
\hline
\end{tabular}


FSL-1 Fibroblast-stimulating lipopeptide-1

GP Glycoprotein

GTN Nitroglycerin

HB Hepatitis B

HMGB1 High mobility group box 1

HOTPR High on-treatment platelet reactivity

HSP Heat shock protein

I/R Ischaemia/reperfusion

ICAM-1 Intracellular adhesion molecule -1

IFN Interferon

IL Interleukin

IL-1R Interleukin 1 receptor

LBP Lipid binding protein

LDLS Low-density lipoproteins

LPS Lipopolysaccharide

LRR Leucine rich repeat

MACE Major adverse cardiovascular events

MCP-1 Monocyte chemoattractant protein -1

MFI Mean fluorescence intensity

MIP-1 $\quad$ Macrophage inflammatory protein-1 $\beta$

MMPs Matrix metalloproteinases

MyD88 Myeloid differentiation primary response protein 88 


\begin{tabular}{|c|c|}
\hline NET & Neutrophil extracellular trap \\
\hline NF-KB & Nuclear factor $\mathrm{kB}$ \\
\hline NSTEMI & Non-ST segment myocardial infarction \\
\hline ODN & Oligodeoxynucleotides \\
\hline PAMPs & Pathogen-associated molecular patterns \\
\hline PARs & Protease activated receptors \\
\hline PBMCs & Peripheral blood mononuclear cells \\
\hline $\mathrm{PCl}$ & Percutaneous coronary intervention \\
\hline PF4 & Platelet factor 4 \\
\hline PGSL-1 & P-selectin glycoprotein ligand 1 \\
\hline PLATO & PLATelet inhibition and patient Outcomes \\
\hline PMAgg & Platelet-monocyte aggregation \\
\hline PPP & Platelet poor plasma \\
\hline PRP & Platelet-rich plasma \\
\hline PRRs & Pattern recognition receptors \\
\hline RANTES & Regulated on Activation, Normal T cell Expressed and Secreted \\
\hline ROS & Reactive oxygen species \\
\hline SCD40L & Soluble CD40 ligand \\
\hline SELECT-ACS & $\begin{array}{l}\text { The Effects of the P-Selectin Antagonist Inclacumab on Myocardial Damage After } \\
\text { Percutaneous Coronary Intervention for Non-ST-Segment Elevation Myocardial } \\
\text { Infarction trial }\end{array}$ \\
\hline STEMI & gment myocardial infarction \\
\hline
\end{tabular}




$\begin{array}{ll}\text { TCR } & \text { T cell receptor } \\ \text { TIR } & \text { Toll/IL-1R } \\ \text { TLRs } & \text { Toll-like receptors } \\ \text { TNF- } \alpha & \text { Tumour necrosis factor } \alpha \\ \text { TRAP6 } & \text { Thrombin receptor activator peptide } 6 \\ \text { TRIF } & \text { Toll/IL-1R (TIR)-domain containing adaptor-inducing interferon } \beta \\ \text { TRITON-TIMI } & \text { The Trial to Assess Improvement in Therapeutic Outcomes by Optimizing Platelet } \\ & \text { Inhibition with Prasugrel-Thrombolysis in Myocardial Infarction trial } \\ \text { TXA2 } & \text { Thromboxane A2 } \\ \text { UA } & \text { Unstable angina } \\ \text { US } & \text { Unstimulated } \\ \text { WB } & \text { Vascular smooth muscles cells } \\ & \end{array}$


1 Chapter 1. Introduction. 


\subsection{The role of platelets in haemostasis and thrombosis.}

Platelets are anucleate, blood-bourne cells that are produced by bone marrow-residing megakaryocytes and remain in circulation for their 8- to 10-day lifespan. Under normal haemostatic conditions, circulating platelets remain quiescent. However, in the presence of vascular injury, platelets play a critical role in thrombosis and function to localize, amplify and sustain the coagulation cascade at the site of injury. As the first line of defence against such injury, platelets adhere to the vessel wall and, from here, platelets become activated, aggregate, and encourage thrombus formation to plug the circulatory leak.

\subsubsection{Platelet adhesion and activation.}

Vascular injury mediates platelet adhesion and activation largely through sub-endothelial exposure of von Willebrand factor (VWF) and collagen. Platelet adhesion is facilitated by expression of a number of adhesion receptors. For example, glycoprotein (GP) Ib-IX-V, which binds to $V W F$, and $\alpha_{2} \beta_{1}$ and $G P V I$, both of which bind to collagen. Once bound, platelets are exposed to, and activate in response to, a multitude of factors that localize to the site of injury. The cumulative action of platelet secretory factors (such as adenosine diphosphate (ADP), thromboxane A2 (TXA2), $\mathrm{Ca}^{2+}$ and serotonin) and of pro-thrombotic factors (such as thrombin and fibrinogen) leads to platelet activation by a number of distinct pathways [1]. These include:

i) Extensive shape change and spreading,

ii) Platelet degranulation, and subsequent expression and secretion of inflammatory mediators such as CD40L and CD62p (P-selectin),

iii) Production of a pro-coagulant phenotype for enhanced thrombin generation, and

iv) Activation of platelet integrin complexes such as GP $\| \mathrm{b} / \mathrm{llla}$, and subsequent aggregation. 
These initial platelet-activating signals are propagated via potent paracrine and autocrine signalling pathways that encourage activation and aggregation of circulating platelets into a growing platelet plug.

\subsubsection{Platelet aggregation.}

Platelet aggregation is initiated by activation and cross-linking of GP IIb/IIla [2]. GP IIb/IIla is one of the most abundant platelet surface receptors [3] and maintains a low affinity for ligand binding when platelets are quiescent. Immediately following platelet adhesion and activation, 'inside-out' signalling converts this receptor to its active state. Fibrinogen, a major circulating coagulation factor, instantly occupies the binding sites of active GP IIb/IIla [4] and acts as a bridging molecule to cross-link adjacent platelets, thus strengthening the growing platelet plug. Following ligand binding, GP IIb/IIla induces 'outside-in' signalling which mediates integrin-cytoskeletal interactions and cytoskeletal rearrangement in order to accelerate platelet aggregation [5]. Platelets, which are spherical when quiescent, extensively reorganize their actin skeleton into spiky spheres with long pseudopod projections. Platelet-platelet interactions are further promoted within the resulting fibrinogen-containing platelet plug by extensive $\mathrm{Ca}^{2+}$-dependent shape change [6]. Shape change converts platelets from an 'adherent' state to an 'aggregation' state, and this further encourages platelet activation and aggregation pathways.

\subsubsection{Thrombus formation.}

The fibrinogen-containing platelet plug, which is formed as a consequence of platelet adhesion, activation and aggregation, serves to propagate local coagulation processes at the site of injury. Platelets encourage thrombin generation by providing an altered, phospholipid-rich surface for cleavage of prothrombin into thrombin, a serine protease. Thrombin generation on the platelet surface also encourages ongoing platelet activation via activation of protease-activated receptors (PARs) 1 and 4 on the platelet surface [7]. Among other functions, the newly generated thrombin 
can cleave soluble fibrinogen into insoluble fibrin to create a fibrin-mesh thrombus. The forming thrombus encapsulates circulating platelets, red blood cells and leukocytes to effectively plug the circulatory leak. Platelets that are newly recruited into this thrombus will signal to platelets deeper within the thrombus to strengthen the adhesive bonds between platelets and the subendothelium, as well as encouraging the ongoing release of platelet mediators. The result is a thrombus that is stabilized against opposing blood flow and that blocks the circulatory leak. 


\subsection{The role of platelets in inflammation.}

Alongside their roles in haemostasis and thrombosis, platelets have emerged as important effectors of the immune system, and their responses have been characterized in both host defence and sterile inflammation. The functions of platelets are often two-pronged: alongside initiating thrombosis, these processes often initiate critical immune responses concurrently. To name a few examples:

i) Platelet activation encourages platelet aggregation and primes the platelet for interaction with leukocytes, and

ii) Platelet degranulation enhances platelet adhesion at the site of vascular injury and releases a multitude of inflammatory mediators.

\subsubsection{Host defence.}

Platelets are increasingly recognized as participants in anti-microbial host defence via direct pathogen detection and via cross-talking with leukocytes [8]. Platelets can directly facilitate pathogenic clearance by various processes including the release of microbicidal proteins [9], pathogen internalization [10] and killing [11]. The important role of platelets in host defence has been demonstrated in clinical and animal models of sepsis. Thrombocytopenia in septic patients [12] and platelet depletion in mouse models of sepsis $[13,14]$ are associated with mortality, and platelet transfusion can ameliorate this effect on survival in mice [13]. A number of studies have shown that the loss of platelets can drive an elevation in plasma TNF $\alpha$ and IL-6 levels in septic mice $[13,15,16]$, and these are cytokines known to be associated with mortality in infection [17]. Interestingly, the loss of a single platelet receptor, GP Ib-IX, is enough to induce elevated levels of plasma TNF- $\alpha$ and IL-6 in septic mice [18]. Furthermore, platelets are known to recruit and interact with various leukocyte subsets to promote leukocyte host defence functions, which include neutrophil extracellular trap (NET) formation [19] and effective antigen presentation [20]. 


\subsubsection{Platelets can modulate leukocyte inflammatory responses.}

It is tempting to suggest that platelets induce predominantly pro-inflammatory responses to infection [21]. However, accumulating evidence suggests that platelets can dampen leukocyte effector functions and this is postulated to be a mechanism to prevent rampant inflammation which, if left unchecked, can cause host damage [22]. Platelets are known to dampen leukocyte cytokine production [23-25] and this dampening effect has also been reported for platelet-derived microparticles [26, 27], indicating both direct and indirect effects of platelets. Some of the dampening effects of platelets seem to be at odds with other pro-inflammatory platelet effects. For example, platelets are able to mediate a reduction of ROS production by neutrophils $[28,29]$, but can also propagate NET formation in response to infectious agents [19]. These findings suggest that platelets can finely balance the induction of both pro-inflammatory and anti-inflammatory responses to infection and inflammation.

The immune functions of platelets are various and diverse; it is likely that the induction of a particular platelet response is a combination of the type of ligand present, the magnitude of the immune response and the type of platelet-leukocyte interaction occurring. In particular, platelets are known to elicit different responses to various agonists [30, 31], which includes the differential release of immunomodulators from their stored granules [32,33]. This suggests an ability to elicit distinct pro-inflammatory or anti-inflammatory responses depending on the type of inflammatory stimulus received by the platelet population. 


\subsection{Cardiovascular disease.}

Cardiovascular disease (CVD) is an umbrella term for any disorder of the heart and blood vessels. CVD remains the leading cause of death in New Zealand and accounts for 30\% of deaths annually, many of which are premature and preventable [34]. Approximately one in 20 adults are diagnosed with heart disease currently, and the flow-on economic and health burden are a substantial health concern. Additionally, Māori and Pacific Island populations feature disproportionately in mortality statistics; Māori are more than twice as likely to die from CVD and 1.5 times more likely to be hospitalized for CVD [34].

Coronary artery disease (CAD) is the clinical manifestation of atherosclerosis that may present clinically as stable angina or as an acute coronary syndrome (ACS), the latter of which is responsible for the majority of deaths from CVD [35]. Following an ACS, these patients have an increased risk of experiencing a repeat event, further confounding health and economic burdens both for the individual and for the New Zealand community. Atherosclerosis and ACS are a product of, among other processes, an intrinsically tight link between pathological thrombotic and inflammatory processes. These processes are discussed in more detail in the below sections.

\subsubsection{Atherosclerosis.}

Atherosclerosis is the underlying cause of CAD, and is characterized by the accumulation of lipid rich plaques within the arterial wall. These atherosclerotic plaques are a complex of lipids, immune cells, endothelial and smooth muscle cells, collagen, and cholesterol crystals, among other components [36]. Atherosclerosis begins to develop in childhood and adolescence [37], and can remain sub-clinical but can also develop into complex and vulnerable plaques. It is the rupture or erosion of these vulnerable atherosclerotic plaques can cause a potentially life-threatening ACS. 
The development of the atherosclerotic plaque is outlined in Figure 1.1. Atherosclerotic plaques preferentially develop at areas of the coronary artery that experience turbulent blood flow [38], which limits endothelial access to nitric oxide (essential for the maintenance of vascular homeostasis), and ultimately results in increased endothelial permeability, leukocyte adhesion and endothelial cell death [39]. Initial atherosclerotic development is characterized by intimal intracellular lipid accumulation and extracellular lipid pooling. Intimal oxidation of low-density lipoproteins (LDLS) results in the release of phospholipids that can pathologically activate endothelial cells [40]. Such induction of chronic endothelial inflammation supports initial atherosclerotic development. Platelet adhesion to the endothelium and platelet-endothelial cell interactions early on in atherosclerotic development allows for leukocyte recruitment and infiltration [41].

Atherosclerosis can be considered an inflammatory disease and is characterized by mass infiltration of monocytes, $T$ cells and dendritic cells and the subsequent production of proinflammatory cytokines and chemokines within the plaque microenvironment $[42,43]$. Leukocyte infiltration is a double-edged sword: for example, as a protective mechanism against excess lipid deposition, monocytes infiltrate into the plaque, differentiate into macrophages and engulf intimal oxidised LDLs. However, excess lipid accumulation within these macrophages induces a switch of these macrophages to pathological foam cells. These lipid-laden foam cells accumulate focally within the intima and initiate the formation of a necrotic core. A growing necrotic core and high burden of infiltrating macrophages are the hallmarks of a vulnerable atherosclerotic plaque [44].

As the plaque continues to grow and push into the lumen, further endothelial disruption results in proliferation of vascular smooth muscle cells (VSMCs) and increased collagen production [45]; a fibrous cap forms. This cap is composed of fibrous collagen and elastin with leukocyte and smooth muscle cell infiltration and is thicker and less cellular than normal intima. This fibrous cap forms a barrier between the thrombogenic plaque contents and the vessel lumen. As these atherosclerotic plaques develop, the artery will initially compensate by undergoing positive remodelling to allow 
normal blood flow to be maintained [46]. Eventually, this arterial remodelling slows down and the atherosclerotic plaque will expand into the lumen and impede blood flow. 


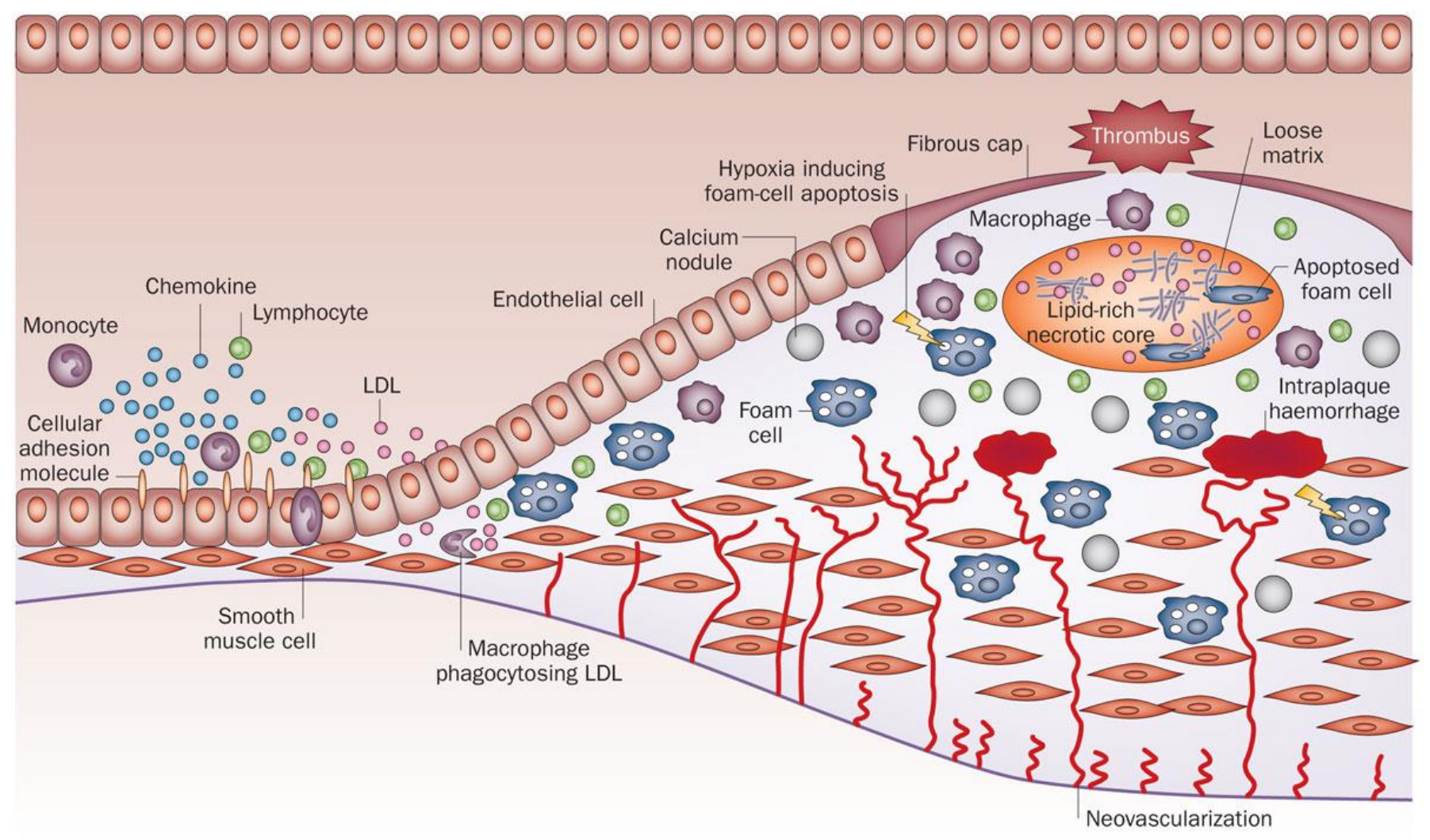

Figure 1.1 The development of the atherosclerotic plaque.

Atherosclerotic plaques form preferentially at areas of turbulent blood flow. The resultant inflammatory microenvironment causes chronic endothelial activation, induces leukocyte recruitment and increased cytokine and chemokine production. Recruited leukocytes and oxidised LDL move through the chronically inflamed endothelium. Recruited monocytes differentiate into macrophages, and macrophages engulf oxidised LDL to become pathological foam cells. Continued foam cell formation and endothelial cell proliferation encourages the formation of a necrotic core underneath a growing plaque. Increased vascular smooth muscle cell proliferation and collagen deposition encourages fibrous cap formation. As atherosclerosis progresses, the coronary arteries stiffen and, eventually, the plaque expands into the lumen. The fibrous cap can become unstable over time, and can erode or rupture. The thrombotic processes that occur at the site of plaque rupture/erosion can manifest clinically as ACS. Image taken with permission from Skeoch et al., 2015 [47]. 


\subsubsection{Acute coronary syndromes (ACS): definitions.}

As atherosclerosis develops, the coronary arteries can become stenotic and obstruct blood flow. This may cause symptoms, most common of which are exertional (anginal) chest pain and dyspnoea (shortness of breath); this is defined clinically as stable angina.

Alternatively, atherosclerotic plaques within the coronary arteries can rupture or erode, causing thrombosis with various degrees of thrombotic occlusion. Plaque rupture/erosion can manifest clinically as an ACS. Sixty to $70 \%$ of ACS cases are characterized by plaque rupture and other cases are characterized by plaque erosion or, less commonly, coronary vasospasm [48]. ACS encompasses the clinical presentation of unstable CAD including: unstable angina (UA), non-STsegment myocardial infarction (NSTEMI) and ST-segment myocardial infarction (STEMI) (Table 1.1). ACS typically presents with prolonged chest pain at rest, and these clinical conditions are characterized by varying degrees of myocardial damage. UA presents clinically as chest pain that occurs with minimal exertion or at rest, on the background of a history of exertional chest pain. UA patients will have electrocardiogram (ECG) changes that are consistent with myocardial ischaemia. UA is differentiated from NSTEMI, as NSTEMI presents as ischaemia that is severe enough to cause detectable leakage of a marker of myocardial injury, such as troponin, into the circulation. The extent of myocardial ischaemia in NSTEMI patients is non-transmural, with associated pathological ECG changes (see Table 1.1). STEMI patients will present with ST-segment elevation on the ECG that is consistent with transmural ischaemia and high circulatory levels of markers of myocardial injury. Collectively, the terms NSTEMI and STEMI can be classed under acute myocardial infarction (AMI). As a result of the improved sensitivity of troponin assays, the incidence of unstable angina is decreasing with a proportionate increase in the incidence of AMI [49]. 
Table 1.1 The spectrum of acute coronary syndromes.

Unstable angina (UA)
$\begin{gathered}\text { Non-ST-segment elevation } \\ \text { myocardial infarction (NSTEMI) }\end{gathered}$

\begin{tabular}{|c|c|c|c|c|}
\hline Exertion & $\begin{array}{l}\text { hal pain that: } \\
\text { Has a more severe onset }\end{array}$ & $\begin{array}{l}\text { Clinical } f \\
\text { angina: }\end{array}$ & eatures of unstable & $\begin{array}{l}\text { Clinical features of myocardial } \\
\text { infarction: }\end{array}$ \\
\hline$\rightarrow$ & $\begin{array}{l}\text { Worsens in } \\
\text { severity/length of } \\
\text { episode }\end{array}$ & & $\begin{array}{l}\text { + elevation of blood } \\
\text { cardiac biomarkers } \\
\text { (e.g. troponin) }\end{array}$ & $\rightarrow+$ ST-segment elevation \\
\hline$\rightarrow$ & $\begin{array}{l}\text { Occurs with minimal } \\
\text { exertion }\end{array}$ & $\rightarrow$ & $\begin{array}{l}\text { +/- ST segment } \\
\text { depression and T wave } \\
\text { flattening/inversion }\end{array}$ & \\
\hline
\end{tabular}

Adapted with permission from Boateng et al., 2013 [50]. 


\subsubsection{Pathology of ACS.}

ACS events are typically characterized by thrombus formation at the site of plaque rupture/erosion [51]. These plaques, known as culprit lesions, are frequently only mildly stenotic prior to plaque rupture/erosion [52]. Consequently, the onset of an ACS is not always preceded by symptoms of angina; rather, the onset of chest pain is often sudden and not dependent on the level of exertion. Atherosclerotic plaques that are most likely to rupture are often referred to as vulnerable plaques. These are characterized by a thin fibrous cap, a large necrotic core, infiltration by inflammatory cells and neovascularization [53]. The fibrous cap thins due to the degradation of collagen (responsible for maintaining mechanical stability) via the action of matrix metalloproteinases (MMPs) [54]. Particularly, overexpression of MMPs has been characterized in plaque-resident monocytes and macrophages, which are key players in atherosclerotic development. This indicates the importance of the presence of these cells for the progression of plaques towards a vulnerable phenotype [55]. Upon rupture/erosion of these plaques, the necrotic core is exposed and various pro-thrombotic components (such as Tissue Factor, collagen fragments and crystalline surfaces) are released into the circulation. Such release initiates and accelerates platelet activation, aggregation and thrombosis at the site of plaque rupture. The platelet-containing thrombus initially begins within the ruptured plaque, distorts the plaque and eventually extends into the lumen. The extent of expansion into the lumen determines the limitation of blood flow to the myocardium and, as a result, the extent of myocardial ischaemia.

It is agreed that plaque rupture/erosion, as an isolated event, may not solely propagate an ACS [56]. Rupture and erosion can occur frequently but does not manifest clinically and, instead, contributes to plaque development [57]. A pro-thrombogenic state, local flow dynamics, and high thrombogenicity of the plaque components is required to propagate plaque rupture/erosion into a coronary event [58]. 


\subsection{The role of platelets in CAD.}

Although critical to maintaining haemostasis during vascular injury, platelet activation and aggregation is pathological in both atherosclerotic development and ACS. Their participation in atherosclerosis and ACS can be explained by 3 processes: i) a pro-thrombotic response to endothelial dysfunction during atherosclerotic development, ii) a pro-thrombotic response to atherosclerotic plaque rupture/erosion, and 3) a pro-inflammatory response to atherosclerosis and ACS.

\subsubsection{Platelets and endothelial dysfunction.}

Endothelial dysfunction is a hallmark of atherosclerotic development and this process can also cause significant alterations in the platelet phenotype, away from their physiological antithrombotic state [59]. During atherosclerotic development, endothelial cells develop a prothrombotic phenotype. A hallmark of this is the accumulation of VWF in their storage granules, which is secreted upon injury and acts as an anchor for circulating platelets [60]. Platelets are also exposed to a multitude of agonists in the dysfunctional endothelial cell microenvironment (such as collagen fibres, ADP and thrombin) [61], and these dysfunctional cells also fail to produce platelet antagonists such as nitric oxide [62] and prostaglandin E1 [63]. Concurrent exposure to various potent agonists combined with the attenuated production of dampening cues can trigger extensive platelet activation at the site of endothelial dysfunction.

\subsubsection{Platelets and thrombosis.}

Atherosclerotic plaque rupture or erosion is a critical event in the occurrence of ACS. The sudden thrombotic response to plaque rupture/erosion is the most severe consequence of atherosclerosis. In a purely pro-thrombotic sense, platelet adherence and activation occurs in reaction to sub-endothelial exposure of a number of pro-thrombotic mediators (as described in Section 1.1). Furthermore, exposition of the lipid-rich and necrotic core into the circulation 
prompts unstable platelet aggregation in circulation which may drive distal embolism or which may be incorporated into a localized fibrin-rich coronary thrombus [64]. Partial or total occlusion of the coronary artery, due to the formation of a platelet-rich thrombus, limits blood flow distally and results in myocardial ischaemia and infarction that manifests clinically as chest pain. A summary of the involvement of platelets in coronary thrombus formation is shown in Figure 1.2. 


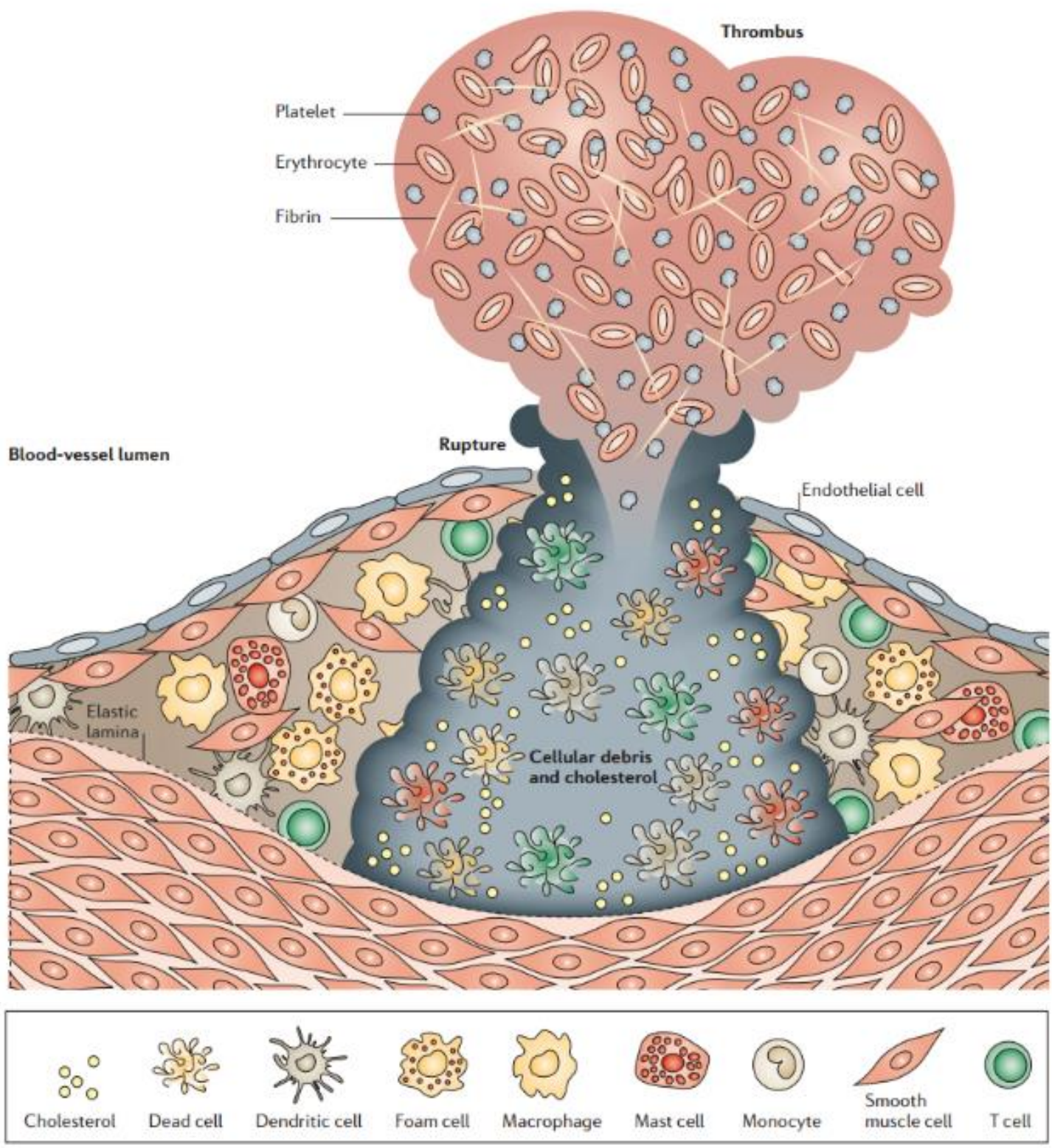

Figure 1.2 Activated platelets contribute to the formation of coronary thrombi during atherosclerotic plaque rupture and ACS.

When the weakened plaque ruptures or erodes, the lipid-rich, necrotic core and sub-endothelium are exposed to procoagulant factors and platelets in circulation. Platelet activation and aggregation occurs rapidly and a fibrin-containing platelet plug initially forms and this is primed for development of a thrombus. This thrombus forms and might occlude the coronary artery, leading to limitation of blood flow distally, and eventual myocardial ischaemia. Image taken with permission from Hansson et al., 2006 [65]. 


\subsubsection{Platelets and inflammation.}

Platelets are increasingly being recognized as critical immune cells in atherosclerosis and ACS. During these processes, platelets upregulate several adhesion molecules that promote plateletendothelial adhesion $[41,66]$ and promote platelet-leukocyte adhesion $[67,68]$. This combination can promote leukocyte and platelet infiltration into the developing atherosclerotic plaque and, during ACS, accumulation into the growing thrombus [69]. The importance of these interactions is evident; knocking out the single platelet adhesion receptor, GP IIb, attenuated atherosclerotic lesion development in the carotid artery of atherosclerotic mice [70]. Importantly, platelet expression of CD62p can advance the inflammatory plaque microenvironment by promoting leukocyte recruitment [71] via binding to leukocyte-expressed P-selectin glycoprotein ligand 1 (PSGL-1). The importance of platelet-CD62p expression for the progression of AMI has also been shown in the clinical setting. The SELECT-ACS trial evaluated the use of inclacumab, a CD62p (Pselectin) antagonist, in NSTEMI patients. The trialists showed a significant reduction in multiple markers of myocardial injury (troponin and CK-MB (creatine kinase-myocardial band)) when inclacumab was administered $<3$ hours before invasive coronary intervention in this patient group $[72,73]$. It is likely that the effect of inclacumab is mediated, in part, by neutralization of CD62pexpressing platelets.

Several studies have reported an increase in circulating platelet-leukocyte aggregates following ACS [74, 75], a process that can be dampened by anti-platelet therapy [76]. Others have also demonstrated the importance of platelet-monocyte aggregation as an early marker of AMI [77]. One important pathological consequence of the formation of these aggregates in AMI may be the initiation and propagation of leukocyte rolling [78]. Leukocytes within these heterotypic aggregates are known to exhibit increased transmigration and endothelial activation when compared to non-conjugated leukocytes [79]. 
CD40 ligand (CD40L) is well characterised as an immune cell activator and has been implicated in the progression of atherosclerosis [80], and by expression and secretion of CD40L, platelets can contribute to the pro-inflammatory milieu surrounding the plaque. Upon platelet activation, surface expression of CD40L can induce endothelial cell and leukocyte secretion of cytokines and chemokines. This secretion, in turn, facilitates leukocyte-endothelial cell interactions [81]. To strengthen these interactions, localized activated platelets also enhance MCP-1 (monocyte chemoattractant protein -1) secretion and ICAM-1 (intracellular adhesion molecule -1) expression by endothelial cells [82]. Furthermore, soluble CD40L release by platelets following activation represents the majority of circulating CD40L [81], and high soluble CD40L levels have been associated with a worse prognosis in STEMI patients [83]. From within the plaque microenvironment or thrombus, platelets can regulate several crucial leukocyte responses such as tissue factor expression [84], fibrin deposition [85] and cytokine and chemokine expression [86, 87].

Aside from CD62p and CD40L, platelet secretion of various other cytokines has also been linked to atherosclerotic progression and ACS. RANTES (Regulated on Activation, Normal T cell Expressed and Secreted) and Platelet Factor 4 (PF4) are released from platelet alpha-granules upon activation and both are considered pro-atherogenic by promoting leukocyte recruitment into the atherosclerotic plaque $[88,89]$. Antagonism of RANTES has also been shown to reduce myocardial injury in a mouse model [90]. Also, IL-1 $\beta$ protein and mRNA is present in resting platelets, upregulated in activated platelets and is known to be critically involved in vascular inflammation and atherosclerosis [91].

A summary of the platelet involvement in atherosclerosis and ACS is shown in Figure 1.3. The evidence presented above suggests that platelets respond to, and contribute to, the inflammatory milieu that is characteristic of atherosclerotic plaque progression to ACS, and act as an interface between inflammation and thrombosis. 
Chapter 1.

Introduction.

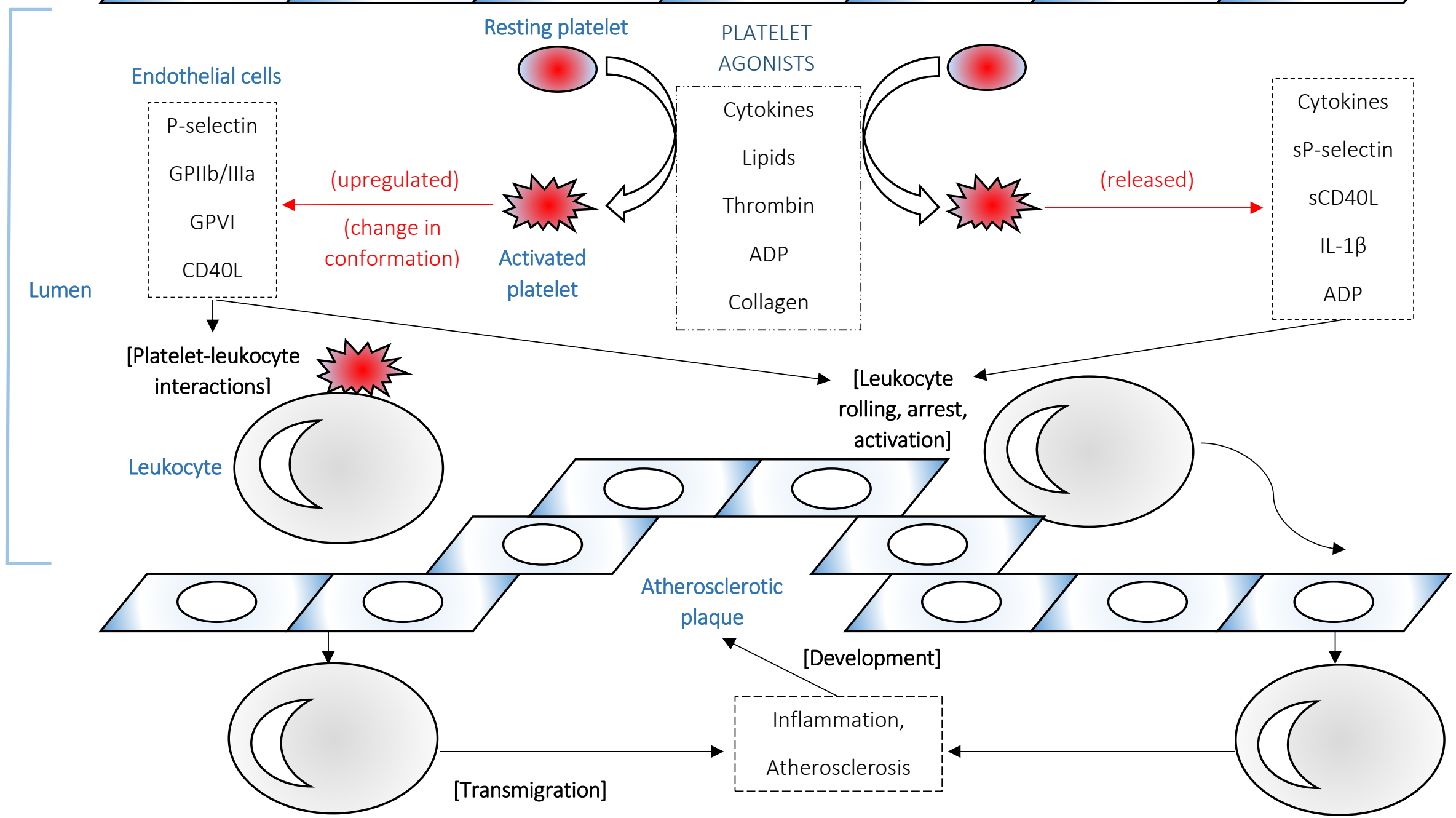

Figure 1.3 Activated platelets contribute to atherosclerosis and ACS.

Activated platelets interact with leukocytes either directly, via upregulation or activation of cell surface receptors, or indirectly, via the release of soluble mediators, to facilitate leukocyte transmigration. Transmigration of leukocytes contributes to the inflammatory milieu that drives atherosclerosis into ACS. Adapted from Lievens et al. [92] with permission. 


\subsection{Treatment of ACS.}

The treatment of patients that present to hospital with AMI is determined by the type of AMI (NSTEMI vs. STEMI, see Table 1.1). Treatment is aimed at reducing myocardial ischaemia and infarction, preserving left ventricular function and optimizing the health outcomes for each patient. Ultimately, treatment reduces the risk of Major Adverse Cardiovascular Events (MACE) such as ventricular fibrillation, heart failure and cardiogenic shock [93].

STEMI is typically characterized by thrombotic occlusion of the artery, causing transmural ischaemia, and prompt reperfusion is indicated in order to return blood flow to the myocardium. Percutaneous coronary intervention $(\mathrm{PCI})$ is an invasive intra-arterial intervention that involves balloon dilation and, usually, the placement of stents in the coronary arteries at sites of severe stenosis or occlusion. In the setting of STEMI, PCI is the treatment of choice to restore and maintain blood flow in order to limit infarct size. Where $\mathrm{PCl}$ is not available or cannot be achieved in a timely manner, thrombolysis with agents such as tenecetaplase is indicated [94]. Timely reperfusion, compared to thrombolytic therapy, increases salvage of the ischaemic myocardium and, therefore, reduces patient morbidity and mortality [95]. NSTEMI is characterized by a smaller, non-transmural ischaemic region and blood flow to the myocardium is present but limited. Within the NSTEMI population, there is a broad spectrum of risk of ischaemia; low-risk patients can be managed conservatively and higher risk patients can be managed invasively. NSTEMI patients are treated with agents such as nitroglycerin and beta-blockers, but if ischaemic symptoms persist, invasive $\mathrm{PCl}$ is indicated [96].

Other adjunctive therapies include analgesia, anti-platelet therapy, hypertensive medication, anticoagulation and statin therapy [93]. The use and effectiveness of anti-platelet therapy (aspirin, clopidogrel, ticagrelor) is discussed in more detail below. 


\subsubsection{Aspirin.}

Aspirin (acetylsalicylic acid) irreversibly inhibits the synthesis of TXA2 by inactivation of the upstream platelet cyclooxygenase-1 (COX1) [97]. Once released by activated platelets, TXA2 plays an important role in encouraging platelet aggregation and is also a potent vasoconstrictor. Aspirin has been shown to reduce the incidence of death and AMI in patients with stable angina [98], unstable angina [99, 100], NSTEMI and STEMI [101]. As such, aspirin is considered a drug-of-choice for secondary prevention of recurrent ischaemic events in ACS patients. The New Zealand guidelines for the management of both NSTEMI [102] and STEMI [103] patients recommend a loading dose of 150-300 mg and a maintenance dose of 75-100 mg daily. This recommendation is consistent with those of the American Heart Association (AHA) $[94,104]$ and European Society of Cardiology (ESC) guidelines [105, 106] (Table 1.2).

\subsubsection{Dual anti-platelet therapy (DAPT).}

For treatment of ACS patients, aspirin is often combined with a $\mathrm{P} 2 \mathrm{Y}_{12}$ receptor antagonist, which blocks platelet activation by ADP (see Table 1.3). The combination of aspirin and a $\mathrm{P}_{2} \mathrm{Y}_{12}$ receptor antagonist, both of which target different mechanisms of platelet activation/aggregation, is known as dual anti-platelet therapy (DAPT). In both patients presenting with ACS and those undergoing $\mathrm{PCl}$, DAPT reduces adverse events when compared to aspirin alone (see, for example, the CURE trial [107]).

\subsubsection{Clopidogrel.}

Clopidogrel is an oral, irreversible, non-competitive $\mathrm{P}_{2} \mathrm{Y}_{12}$ receptor antagonist that is paired with aspirin for DAPT (Table 1.3). Clopidogrel is metabolized by the hepatic cytochrome P (CYP) 450 system of enzymes to generate a highly unstable active metabolite (R-130964). R-130964 exerts its anti-platelet effects by binding to platelets that are trafficked through the hepatic circulation 
and irreversibly inhibits ADP-mediated platelet activation and aggregation. DAPT with clopidogrel has been demonstrated to be a more effective therapy for secondary prevention than aspirin alone. The CURE [107], CREDO [108] and CLARITY [109] trials, all of which compared DAPT with clopidogrel against aspirin monotherapy, reported reductions in MACE (a composite of death, AMI and stroke at varying follow-up times) in the DAPT with clopidogrel arm. Clopidogrel, at a loading dose of 300-600 mg and 75-100 mg maintenance dose is recommended in addition to aspirin for the treatment of ACS by the New Zealand [102, 103] and international guidelines [94, 104-106] groups (Table 1.2).

\subsubsection{Ticagrelor.}

Ticagrelor is an alternative oral $\mathrm{P} 2 \mathrm{Y}_{12}$ receptor antagonist that is paired with aspirin for treatment of ACS. Unlike clopidogrel, ticagrelor is a reversible and direct acting antagonist (see Table 1.3). These properties have translated clinically into more effective anti-platelet treatment when compared to DAPT with clopidogrel. In stable CAD patients, ticagrelor (180 mg loading /90 mg daily) produced greater and more rapid platelet inhibition when compared to clopidogrel (600 mg loading/75 mg daily) [110]. More specifically, mean platelet inhibition of greater than a 50\% fall from baseline was seen in 98\% of patients treated with ticagrelor 2 hours after administration, compared with just 31\% in clopidogrel-treated patients. Most importantly, the PLATelet inhibition and patient Outcomes (PLATO) trial demonstrated more effective protection against MACE following DAPT with ticagrelor compared to DAPT with clopidogrel in ACS patients [111]. In this trial, the rate of $\mathrm{AMI}$ (5.8\% in ticagrelor-treated vs. 6.9\% in clopidogrel-treated patient group, $p=0.005)$, death from vascular causes $(4.0 \%$ vs. $5.1 \%, p=0.001)$ and death from any cause $(4.5 \%$ vs. 5.9\%, p<0.001) were all attenuated following DAPT with ticagrelor, in comparison to DAPT with clopidogrel [111]. There was no significant difference in the rates of major bleeding events between the ticagrelor (11.6\%) and clopidogrel $(11.2 \%, p=0.43)$, but there was a small increase in non-coronary artery bypass graft (CABG) bleeding [111]. Following this landmark trial, the New Zealand [102, 103] and international guidelines [104-106] for the treatment of ACS recommend the use of DAPT with ticagrelor over DAPT with clopidogrel. Under New Zealand and international 
guidelines, ticagrelor is administered as a $180 \mathrm{mg}$ loading dose followed by a $90 \mathrm{mg}$ twice-daily maintenance dose (Table 1.2).

\subsubsection{Prasugrel.}

Prasugrel is a potent irreversible $\mathrm{P}_{2} \mathrm{Y}_{12}$ receptor antagonist that is available for treatment of ACS. The TRITON-TIMI 38 trial established the superiority of DAPT with prasugrel over DAPT with clopidogrel in ACS patients undergoing $\mathrm{PCl}$, where the primary end-point was a composite of death from cardiovascular causes, non-fatal AMI or non-fatal stroke [112]. For patients receiving prasugrel, there was a attenuated rate of recurrent $\mathrm{AMI}$, a reduction in the rate of revascularization of the target vessel, and a reduction in the rate of stent thrombosis when compared with patients receiving clopidogrel. However, treatment with prasugrel was also associated with a higher risk of life-threatening and fatal bleeding.

Whilst prasugrel is approved for use in New Zealand, this $\mathrm{P}_{2} \mathrm{Y}_{12}$ receptor antagonist is not funded by the Pharmaceutical Management Agency (PHARMAC) for routine use in the treatment of ACS. In the New Zealand setting, ACS patients predominantly receive aspirin paired with either clopidogrel or ticagrelor. 
Table 1.2 Guidelines for the treatment of ACS with oral anti-platelet agents.

\begin{tabular}{|c|c|c|c|}
\hline UA/NSTEMI & STEMI & & \\
\hline ESC [105] AHA/ACC [104] & $\begin{array}{l}\text { NZ arm of } \\
\text { CSANZ [102] }\end{array}$ & AHA/ACC [94] & $\begin{array}{l}\text { NZ arm of } \\
\text { CSANZ [103] }\end{array}$ \\
\hline
\end{tabular}

\begin{tabular}{|c|c|c|c|c|c|c|}
\hline $\begin{array}{l}\text { Aspirin } \\
\text { indefinitely }\end{array}$ & $\mathrm{IA}$ & $\mathrm{IA}$ & $1 A^{e}, 1 C^{f}$ & $\mathrm{IA}$ & IA & $\mathrm{IA}$ \\
\hline $\begin{array}{l}\text { DAPT up to } \\
12 \text { months }\end{array}$ & $\mathrm{IA}$ & Recommended & $I A$ & IC & Recommended & $\begin{array}{ll}-- \\
\end{array}$ \\
\hline Clopidogrel & $\mathrm{IB}^{\mathrm{a}}$ & $|B,| B^{c}$ & $\left\|A^{c},\right\| a B^{a}$ & $\left|C^{g},\right| B^{c}$ & I B & $I C^{a}$ \\
\hline Ticagrelor & $\mathrm{I}^{\mathrm{b}}$ & $\mid \mathrm{B}, \mathrm{\| la} \mathrm{B}^{\mathrm{d}}$ & I B & I B & I B & I B \\
\hline
\end{tabular}

UA, unstable angina; NSTEMI, non-ST-segment elevation myocardial infarction; STEMI, ST-segment elevation myocardial infarction; ESC, European Society of Cardiology; AHA/ACC, American Heart Association/American College of Cardiology; CSANZ, Cardiology Society of Australia and New Zealand.

a if other $\mathrm{P}_{2} \mathrm{Y}_{12}$ receptor antagonists are unavailable or contraindicated, ${ }^{\mathrm{b}}$ when a patient is at moderate-to-high risk of an ischaemic event, ${ }^{\mathrm{c}}$ as an alternative to aspirin in patients who are intolerant to aspirin, ${ }^{\mathrm{d}}$ in preference to clopidogrel for patients with an early invasive or ischaemia-guided strategy, ${ }^{e}$ immediate administration, ${ }^{\mathrm{f}}$ indefinite administration, $\mathrm{g}$ preferably when prasugrel or ticagrelor are not available or contraindicated.

\section{Classes of recommendation:}

Class I: Benefits >> Risk. Procedure/treatment should be performed/administered.

Class Ila: Benefits >> Risk. Additional studies with focused objectives needed; it is reasonable to perform procedure/administer treatment.

Class III: Evidence/general agreement that the given treatment or procedure is not useful/effective, and in some cases, may be harmful.

\section{Levels of Evidence:}

Level A: Multiple populations evaluated; data derived from multiple randomized clinical trials or meta-analyses Level B: Limited populations evaluated; data derived from a single randomized trial or nonrandomized studies Level C: Very limited populations evaluated; only consensus opinion of experts, case studies, or standard of care. 
Table 1.3 $\mathrm{P}_{2} \mathrm{Y}_{12}$ receptor antagonists.

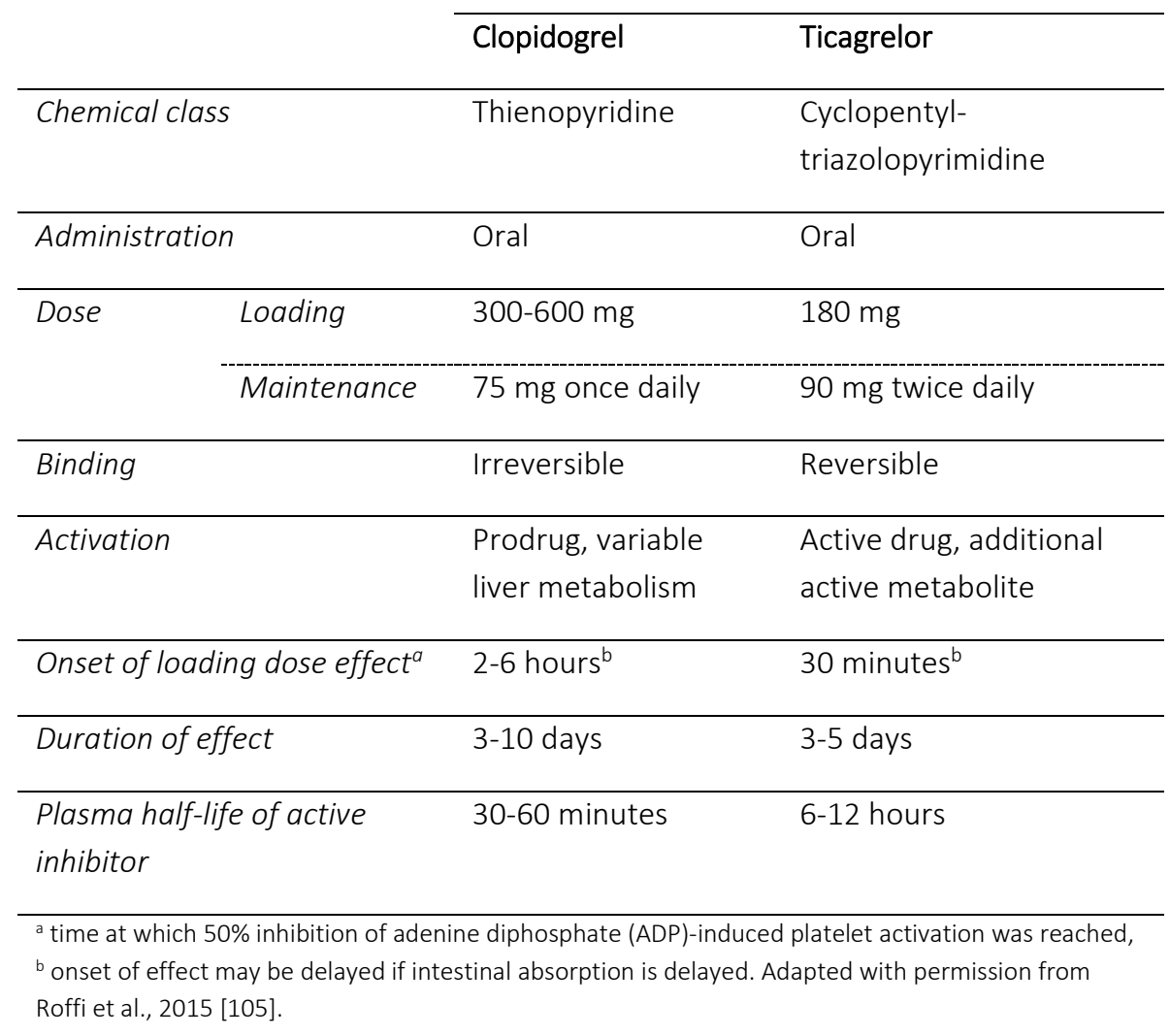




\subsubsection{Residual platelet activation in AMI patients.}

Despite treatment with DAPT, residual platelet activation and aggregation has been observed. High on-treatment platelet reactivity (HOTPR) to ADP has been consistently and robustly associated with the development of ischaemic events $[113,114]$. HOTPR has been noted in ACS populations treated with both clopidogrel and ticagrelor. Extreme variability in platelet inhibition following clopidogrel administration has been reported $[115,116]$. In post- $\mathrm{PCl}$ patients with a attenuated responsiveness to clopidogrel (those patients falling into the first quartile when stratified according to percentage reduction in ADP-mediated platelet aggregation), a $40 \%$ rate of recurrent cardiovascular events was recorded as opposed to a 0\% rate recorded for quartiles 3 and 4 [117]. In another study, HOTPR despite treatment with clopidogrel was significantly associated with cardiovascular death $(2.8 \%$ vs. $0 \%, p=0.04)$ and stent thrombosis $(4.6 \%$ vs. $0 \%$, $p=0.004)$ [118]. Although the rates are lower than reported for clopidogrel, patients can still experience HOTPR despite treatment with ticagrelor. Our laboratory group has previously reported a $16 \%$ rate of HOTPR in AMI patients undergoing invasive management [119] and Verdoia et al. [120] reported a 13\% rate of HOTPR in a population of $\mathrm{PCl}-\mathrm{ACS}$ patients. However, others have reported that only a low percentage (1.5\%) of a pooled patient population experience HOTPR with ticagrelor [121].

Residual platelet reactivity while on DAPT is likely to be due to a combination of factors, such as patient non-compliance, co-morbidities and drug pharmacodynamics [122]. It has also been shown that platelets retain their responsiveness to agonists that are not therapeutically targeted by DAPT $[123,124]$. For example, platelet activation and aggregation was still noted following treatment with various $\mathrm{P}_{2} \mathrm{Y}_{12}$ receptor antagonists and stimulation with collagen, epinephrine and thrombin receptor activator peptide (TRAP)-6 [123, 124]. This is highly clinically relevant, given the multitude of pathways by which platelets can become activated in the setting of ACS. If platelets continue to respond to pathological signals in the aftermath of an ACS and despite treatment, there continues to be a risk of recurrent thrombosis in these patients. 


\subsection{Alternative pathways of platelet activation: platelets and infection.}

The mechanisms underlying ACS events are complex and include increased coronary and systemic inflammation coupled with increased pro-thrombotic processes. We hypothesize that current anti-platelet medication, which targets two well-known pro-thrombotic pathways (COX-1/TXA2, $A D P)$, may not adequately dampen down the many actions of platelets in driving both thrombotic and inflammatory responses during ACS. Infection may be an alternative pathway of platelet activation by which platelets may still become activated post-ACS, despite treatment with DAPT.

Pathogens are known to activate platelets via various indirect and direct mechanisms. For example, platelet aggregation was shown in response to the common oral bacterium Streptococcus sanguis [125] and Staphylococcus aureus [126]. Similarly, platelets that are exposed to Staphylococcal $\alpha$ toxin release PF4 and induced aggregation [127]. Aerococcus urinae, which has the capacity to cause urinary tract infections and infectious endocarditis, has been demonstrated to induce platelet activation and aggregation [128]. Platelet aggregation in response to $1 \mu \mathrm{M}$ ADP was significantly higher in a cohort of patients with suspected viral upper respiratory tract infections (76\% aggregation) when compared to both healthy controls (22\%) and to the 6 -week follow-up results $(26 \%)[129,130]$. The percentage of circulating CD62p-positive platelets increased in Helicobacter pylori infected wild type mice when compared to healthy controls [131]. Similar results were recorded for $H$. pylori patients [132] and direct incubation of H. pylori with platelets causes activation (increased CD62p), increased apoptosis (increased Annexin $\vee$ binding) and high levels of adhesion with the bacteria [133]. Infection characteristically establishes a pro-inflammatory response in the host and, in this manner, is likely to indirectly activate platelets. Systemic inflammation often precedes or accompanies the occurrence of an ACS [134] and elevation of blood-borne inflammatory biomarkers has been demonstrated to be predictive of further ischaemic events [135]. 


\subsection{Toll-like receptors (TLRs): the first effectors of the response to infection.}

Toll-like receptors (TLRs) are a group of pattern recognition receptors (PRRs) that are constitutively expressed on immune cells and act as sentinels against both exogenous and endogenous 'danger' signals. TLRs are the 'first line of defence' against broad, microbe-specific pathogen-associated molecular patterns (PAMPs). TLR activation during infection sets in motion a series of molecular events that leads to initiation of the innate immune system followed by development of acquired immunity.

Mammalian TLRs were discovered following the discovery of the Toll receptor in the Drosophila fly. A mutation in this Drosophila receptor confered severe susceptibility to fungal infection by inhibiting the production of defensive anti-fungal peptides [136]. The human homologue, TLR4, was subsequently identified [137]. The mechanism by which TLR4 induces innate immune responses to infection was shown to involve nuclear factor-kB (NF- KB) activation and subsequent production of NF-KB-controlled pro-inflammatory cytokines [137]. NF-KB is the 'master controller' of inflammation and, in activating this transcription factor, TLRs rapidly induce inflammation in response to infection.

Although classically characterized as responding to PAMPs, TLRs are also increasingly recognized as responding to host-derived danger-associated molecular patterns (DAMPs). Also known as 'alarmins', these molecular patterns are released by stressed and necrotic cells to perpetuate a non-infectious, sterile inflammatory response which ultimately mediates tissue repair. Increased circulating levels of DAMPs have been associated with many inflammatory diseases $[138,139]$ and binding of DAMPs to TLRs can lead to a self-sustaining auto-inflammatory response [140, 141]. This suggests that the TLR response to DAMPs can initiate a long-lasting, heightened proinflammatory state. 


\subsubsection{Sub-cellular localization.}

Within the mammalian TLR family, there are 10 identified receptors which can be divided into 2 subpopulations by their cellular localization and, hence, by their PAMP/DAMP specificity. TLR1, 2, 4, 5, 6 and 10 are expressed on the cell-surface and traditionally respond to extracellular lipid molecular patterns. TLR3, 5, 7, 8 and 9 are expressed in the endosome, lysosome or endoplasmic reticulum of immune cells and traditionally detect microbial nucleic acid components that are a by-product of microbial digestion.

\subsubsection{Structure and signalling.}

TLRs are Type I transmembrane proteins (these proteins contain an extracellular N-terminal) and are characterized by the presence of 19-25 tandem copies of the leucine-rich repeat (LRR; $x \operatorname{xx} \operatorname{Lx} \operatorname{Lx} x)$ in their extracellular N-terminal domain. This $\mathrm{N}$-terminal domain of TLRs is responsible for recognizing their respective ligands. The unique structure of the LRR domain forms a distinctive 3D horseshoe-like structure. Following ligand binding, TLRs undergo dimerization and these horseshoe structures come together to sandwich the ligand. Most TLRs transduce their signal via homodimerization following ligand binding. However, TLR2 is unique in that this receptor will heterodimerize with either TLR1 or TLR6 to recognize different bacterial lipopeptides. Conformational shape change of the receptor complex occurs following dimerization and is required for signal transduction. The TLR intracellular domain is homologous to the Interleukin (IL)-1 receptor (IL-1R), thus named the Toll/IL-1R (TIR) domain, and is essential for downstream signalling. Engaging these receptors initially activates various TIR-domain containing adaptor proteins and, secondarily, the signal is transduced to various kinases and ubiquitin ligases. Ultimately, signal transduction results in nuclear translocation of NF-kB, among other transcription factors and the production of pro-inflammatory cytokines and type I interferons (IFNs). 


\subsubsection{Ligand binding.}

The ligand binding properties of a subset of TLRs (TLR1, 2, 4, 6 and 9) which were examined in this thesis are discussed below. There is consensus in the literature that this TLR subset is expressed on and/or within healthy human platelets (also discussed in Section 1.10), and this was the basis for choosing to study these receptors in this thesis. The expression patterns of other TLRs on and within platelets are less well-characterized and were not studied here. Further discussion of the TLR family is restricted to this subset.

\subsubsection{TLRs 1, 2 and 6.}

TLR2 is essential for the recognition of bacterial lipopeptides through the association of TLR1 and 6, which are both highly structurally homologous to TLR2 [142]. TLR1 and 6 allow for the subtle discrimination between diacylated (TLR2/6 agonist) and triacylated (TLR2/1 agonist) bacterial lipopeptides. The TLR2-mediated inflammatory responses to bacterial lipopeptides is enhanced by association with co-factor molecules such as CD14 [143] and CD36 [144] as well as coupling with other PRRs such as dectin [145]. Aside from reacting to exogenous PAMPs, TLR2 is known to respond to various DAMPs. For example, high mobility group box 1 (HMGB1) is a late cytokine mediator released from immune cells which utilizes the TLR2 signalling pathway [146]. Similarly, heat-shock protein (HSP) 70, which is upregulated following exposure to a variety of stressful stimuli, can elicit a pro-inflammatory response through TLR2 [147].

\subsubsection{TLR4.}

TLR4 is perhaps the most well-studied TLR and induces potent innate immune responses to lipopolysaccharide (LPS), which is a PAMP that constitutes approximately $75 \%$ of the outer membrane of Gram-negative bacteria [148]. TLR4 interacts with LPS with the participation of various other molecules. Lipid binding protein (LBP) is an acute phase protein that is rapidly produced following an inflammatory challenge [149] and shuttles LPS to CD14, which is either 
soluble or cell surface-bound, and this complex facilitates the transfer of LPS to TLR4, which is complexed to MD-2. MD-2, in complex with TLR4, confers responsiveness of TLR4 to LPS [150]. Thus, a large complex of proteins is required for effective TLR4 signalling in response to LPS. TLR4 expression increases on monocytes during LPS challenge [151] and during sepsis [152], and these results indicate that cells can dynamically and rapidly respond to infection via the TLR4 pathway. TLR4 has also been shown to respond to HMGB1 [146] and HSP70 [147], alongside TLR2, as well as to other DAMPs including hyaluronic acid [153] and fibrinogen [154].

\subsubsection{TLR9.}

Bacterial DNA is known to induce pro-inflammatory effects on mammalian immune cells [155] and these effects are dependent on the presence of unmethylated cytidine-guanosine (CpG) dinucleotides in prokaryotic DNA. CPG dinucleotides are under-represented and methylated in vertebrate DNA, such that the immune-stimulatory activity is specific to prokaryotic DNA [156]. TLR9 was shown to mediate the response to prokaryotic CpG; TLR9-deficient mice showed no elevation of serum pro-inflammatory cytokines and are resistant to the lethal effects of CpG DNA [156]. TLR9 localizes in the endoplasmic reticulum of immune cells and is translocated to compartments containing CpG DNA following immune cell exposure to infection [157]. TLR9 has also been shown to respond to tissue necrosis and exacerbate the innate immune response to ischaemia [158]. For example, endogenous extracellular histones can function as immunogenic DAMPs by activating the TLR9 pathway [159], and have previously been shown to act as mediators of death in sepsis [160]. 


\subsection{TLRs and CAD.}

Activation of TLRs in CAD is two-pronged: CAD is likely to be influenced by both systemic and localized infection (PAMPs) and influenced by the release of 'danger' self-ligands by both the ruptured atherosclerotic plaque and infarcted myocardium (DAMPs).

\subsubsection{TLRs and atherosclerosis.}

Atherosclerotic progression is primarily an inflammatory disease and is heavily dependent on sustained inflammation at the plaque site. As a host defence mechanism, TLR-mediated inflammation is generally beneficial in limiting infection although the response of TLRs to both PAMPs and DAMPs can contribute to the atherosclerotic pathogenesis. See Table 1.4 for a summary of the involvement of TLRs in atherosclerosis.

TLRs were first shown to be involved in atherosclerosis by Bjorbacka et al. [161]. Total body deficiency of MyD88 (Myeloid differentiation primary response 88), a downstream signalling molecule of the TLR signalling pathway, attenuated the development of atherosclerosis via a reduction in macrophage accumulation, and a subsequent reduction in cytokine and chemokine release, at the plaque site in atherosclerotic $\left(\mathrm{ApoE}^{-/}\right)$mice [161]. These findings have been confirmed by others [162].

Most of the current literature has focussed on the contribution of TLR2 and 4 to atherosclerosis. Total-body deficiency of TLR2 [163-165] and 4 [162] produce the same protective phenotype described for MyD88-deficient mice. Interestingly, decreased plasma cholesterol was noted in atherosclerotic-prone, TLR2-deficient mice [165] suggesting a role of TLR2 for regulating cholesterol. Increased endothelial TLR2 expression has been noted in areas of disturbed, compared to laminar, flow and this region was associated with an increase in leukocyte infiltration 
and lipid accumulation [166]. Atherosclerotic plaques occur at regions of disturbed blood flow [167] and, via this mechanism, TLR2 may regulate plaque development at these sites. Increased TLR4 expression was noted in lipid-rich plaques of human coronary arteries, and a majority of these TLR4-positive cells were identified as macrophages [168, 169]. Furthermore, there was a significant and dose-dependent increase in macrophage TLR4 mRNA in response to oxidised LDL, a chief component of atherosclerotic plaques [168]. The latter results suggest the importance of macrophage TLR4 for atherosclerotic development.

Less is known about how TLR1 and 6 influence atherosclerosis, although one study has reported on their effects. Agonizing wildtype mice with the prototypical TLR2/1 agonist, Pam3CSK4, significantly increased the size of aortic lesions when compared to TLR1-deficient mice [170], which suggests that agonizing TLRs can encourage atherosclerosis. The same authors also reported increased heart valve lesions and interleukin release following MALP2 agonism (TLR2/6 agonist) in wildtype mice when compared to TLR6-deficient mice [170]. The effect of TLR9 on the progression of atherosclerosis remains understudied. 
Table 1.4 Evidence for involvement of TLRs in atherosclerosis.

\begin{tabular}{|c|c|c|c|c|}
\hline TLR & $\begin{array}{l}\text { Author } \\
\text { [reference] }\end{array}$ & Methods (test vs. control) & Results & $\begin{array}{l}\text { TLR pro-atherogenic or } \\
\text { athero-protective? }\end{array}$ \\
\hline \multirow[t]{2}{*}{1} & $\begin{array}{l}\text { Edfeldt et } \\
\text { al., } 2002 \\
{[171]}\end{array}$ & $\begin{array}{l}\text { Human atherosclerotic } \\
\text { plaques (obtained by carotid } \\
\text { endarterectomy) vs. internal } \\
\text { mammary artery (CABG). }\end{array}$ & $>3$-fold $\uparrow$ TLR1 mRNA transcript level in atherosclerotic plaques. & Pro-atherogenic. \\
\hline & $\begin{array}{l}\text { Curtiss et } \\
\text { al., } 2012 \\
{[170]}\end{array}$ & 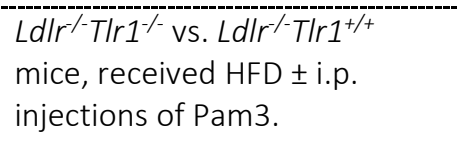 & 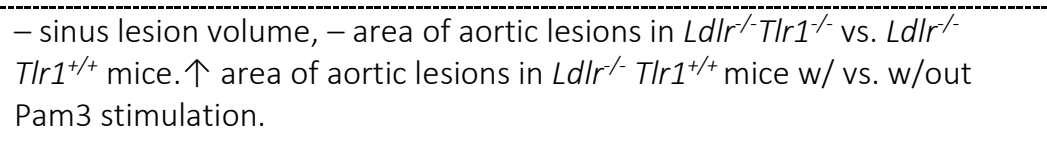 & Pro-atherogenic. \\
\hline \multirow[t]{5}{*}{2} & $\begin{array}{l}\text { Mullick et } \\
\text { al., } 2005 \\
{[172]}\end{array}$ & 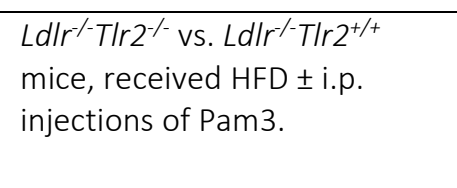 & $\begin{array}{l}\downarrow \text { total plasma cholesterol, } \downarrow \text { aortic atherosclerosis, } \downarrow \text { aortic valve } \\
\text { lesion size in } L d l r^{--} T / r 2^{-/} \text {vs. } L d l r^{-/-} T / r 2^{+/+} \text {mice. } \uparrow \text { area of aortic lesions, } \uparrow \\
\text { aortic sinus lesion volume in } L d / r^{--} T / r 2^{+/+} \text {mice } w / \text { Pam3 stimulation vs. } \\
L d l r^{--} T / r 2^{-/-} \text {mice. }\end{array}$ & Pro-atherogenic. \\
\hline & $\begin{array}{l}\text { Mullick et } \\
\text { al., } 2008 \\
{[166]}\end{array}$ & $\begin{array}{l}\text { Ldlr } r^{-/} T / r 2^{-/-} \text {vs. } L d l r^{-/-T / r 2^{+/+}} \\
\text {mice, received HFD. }\end{array}$ & $\begin{array}{l}\uparrow \text { endothelial TLR2 expression in lesser curvature of aorta (area of } \\
\text { disturbed flow) in } L d l r^{--} T / r 2^{+/+} \text {mice. } \downarrow \text { leukocyte infiltration, } \downarrow \text { lipid } \\
\text { accumulation, } \downarrow \text { pathological change in endothelial cell morphology in } \\
\text { the lesser curvature of the aorta in } L d / r^{-/-} T / r 2^{-/-} \text {vs. } L d / r^{-/} T / r 2^{+/+} \text {mice. }\end{array}$ & $\begin{array}{l}\text { TLR2 expression is pro- } \\
\text { atherogenic in areas of } \\
\text { disturbed flow. }\end{array}$ \\
\hline & $\begin{array}{l}\text { Dunzendor } \\
\text { fer et al., } \\
2004 \text { [173] }\end{array}$ & $\begin{array}{l}\text { HCAECs exposed to laminar vs. } \\
\text { disturbed flow. }\end{array}$ & $\begin{array}{l}\downarrow \text { TLR2 protein + mRNA expression, } \downarrow \text { responsiveness to MALP-2 in } \\
\text { HCAECs under laminar vs. disturbed flow. }\end{array}$ & $\begin{array}{l}\text { TLR2 suppression is } \\
\text { athero-protective in } \\
\text { areas of laminar flow. }\end{array}$ \\
\hline & $\begin{array}{l}\text { Edfeldt et } \\
\text { al., } 2002 \\
{[171]}\end{array}$ & $\begin{array}{l}\text { Human atherosclerotic } \\
\text { plaques (obtained by carotid } \\
\text { endarterectomy) vs. internal } \\
\text { mammary artery (CABG). }\end{array}$ & $\begin{array}{l}>3 \text {-fold } \uparrow \text { TLR2 mRNA transcript level in atherosclerotic plaques. } \\
\text { TLR2 expressed in macrophages and endothelial cells. }\end{array}$ & Pro-atherogenic. \\
\hline & $\begin{array}{l}\text { Madan et } \\
\text { al., } 2008 \\
{[164]}\end{array}$ & $\begin{array}{l}\text { Apoe }{ }^{+/-} \text {TIr } 2^{-/-} \text {vs. Apoe } e^{+/-} \text {Tlr } 2^{+/+} \\
\text {mice, received a HFD }+ \text { i.v. } \\
\text { injections of } P \text {. gingivalis or } \\
\text { FSL-1. }\end{array}$ & 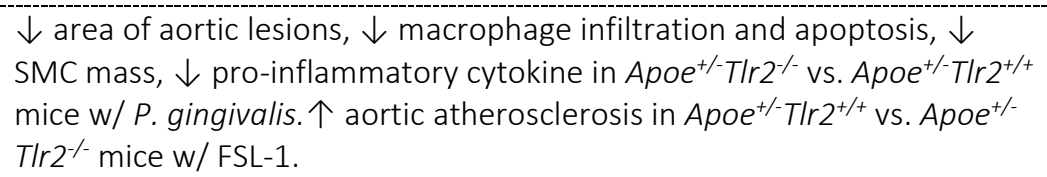 & Pro-atherogenic. \\
\hline
\end{tabular}


Table 1.4 Evidence for involvement of TLRs in atherosclerosis (continued).

\begin{tabular}{|c|c|c|c|c|}
\hline 2 & $\begin{array}{l}\text { Monaco et } \\
\text { al., } 2009 \\
{[174]}\end{array}$ & $\begin{array}{l}\text { Atheroma cells (obtained from } \\
\text { carotid endarterectomy) } \\
\text { cultured } \pm \text { TLR2 neutralizing } \\
\text { antibody. }\end{array}$ & $\begin{array}{l}\downarrow \text { NF-kB activation, } \downarrow \text { MCP-1, } \downarrow \text { IL-6 and }-8, \downarrow \text { MMP-1, 2, 3, } 9 \\
\text { production in TLR2-neutralized vs. non-TLR2-neutralized cells. }\end{array}$ & Pro-atherogenic. \\
\hline \multirow[t]{5}{*}{4} & $\begin{array}{l}\text { Michelsen } \\
\text { et al., } 2004 \\
\text { [162] }\end{array}$ & $\begin{array}{l}\text { Apoe }^{-/-} \text {T/r4 }{ }^{-/-} \text {vs. Apoe } \\
\text { mice, received HFD. }\end{array}$ & $\begin{array}{l}\downarrow \text { total atherosclerotic lesion area, } \downarrow \text { lipid and macrophage } \\
\text { accumulation in aortic sinus plaques, } \downarrow \text { circulating levels of pro- } \\
\text { inflammatory cytokines in Apoe } e^{-/-} T / r 4^{-/-} \text {vs. Apoe } e^{-/-T / r 4^{+/+}} \text {mice. }\end{array}$ & Pro-atherogenic. \\
\hline & $\begin{array}{l}\text { Vink et al., } \\
2002 \text { [169] }\end{array}$ & $\begin{array}{l}\text { Human coronary artery } \\
\text { segments (post-mortem). }\end{array}$ & $\begin{array}{l}\text { TLR4 expression in the adventitial cells of atherosclerotic arteries, TLR4- } \\
\text { positive cells identified as macrophages and fibroblasts. }\end{array}$ & Pro-atherogenic. \\
\hline & $\begin{array}{l}\text { Xu et al., } \\
2001[168]\end{array}$ & $\begin{array}{l}\text { Human coronary artery } \\
\text { segments (post-mortem). }\end{array}$ & $\begin{array}{l}\uparrow \text { TLR4 expression in lipid-rich plaques, - expression in fibrous plaques. } \\
\text { TLR4-positive cells identified as macrophages. } \\
\uparrow \text { macrophage TLR4 mRNA dose-dependently in response to oxLDL. }\end{array}$ & Pro-atherogenic. \\
\hline & $\begin{array}{l}\text { Edfeldt et } \\
\text { al., } 2002 \\
{[171]}\end{array}$ & $\begin{array}{l}\text { Human atherosclerotic } \\
\text { plaques (obtained by carotid } \\
\text { endarterectomy) vs. internal } \\
\text { mammary artery (CABG). }\end{array}$ & $\begin{array}{l}>3 \text {-fold } \uparrow \text { TLR4 mRNA transcript in atherosclerotic plaques. } \\
\text { TLR4 primarily expressed by macrophages and endothelial cells. }\end{array}$ & Pro-atherogeinc. \\
\hline & $\begin{array}{l}\text { Kiechlet } \\
\text { al., } 2002 \\
{[175]}\end{array}$ & $\begin{array}{l}\text { Screening for the Asp299Gly } \\
\text { LOF polymorphism of TLR4 + } \\
\text { progression of carotid } \\
\text { atherosclerosis assessed in } \\
810 \text { people. }\end{array}$ & $\begin{array}{l}\downarrow \text { pro-inflammatory cytokine levels, } \downarrow \text { acute-phase reactants, } \downarrow \text { soluble } \\
\text { adhesion molecules, } \uparrow \text { susceptibility to severe bacterial infections, } \downarrow \text { risk } \\
\text { of carotid atherosclerosis in subjects with } 1 \text { or more of the Asp299Gly } \\
\text { TLR4 allele. }\end{array}$ & Pro-atherogenic. \\
\hline 6 & $\begin{array}{l}\text { Curtiss et } \\
\text { al., } 2012 \\
{[170]}\end{array}$ & 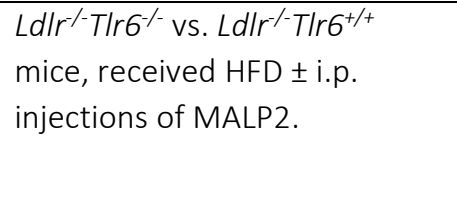 & 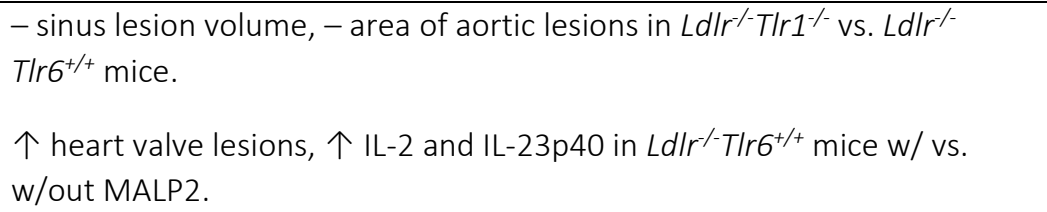 & Pro-atherogenic. \\
\hline
\end{tabular}


Table 1.4 Evidence for involvement of TLRs in atherosclerosis (continued).

\begin{tabular}{|c|c|c|c|c|}
\hline \multirow[t]{2}{*}{ MyD88 } & $\begin{array}{l}\text { Bjorkbacka } \\
\text { et al., } 2004 \\
{[161]}\end{array}$ & $\begin{array}{l}\text { Apoe }^{-/-} M y D 88^{-/-} \text {vs. Apoe }{ }^{-/-} \\
M y D 88^{+/+} \text {mice, received HFD. }\end{array}$ & 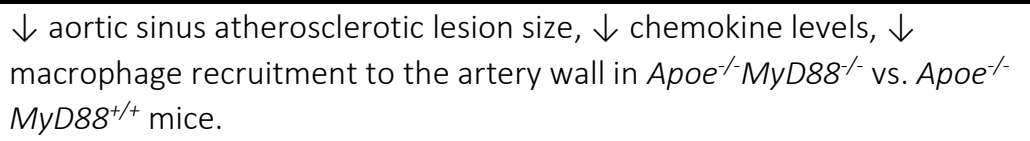 & Pro-atherogenic. \\
\hline & $\begin{array}{l}\text { Michelsen } \\
\text { et al., } 2004 \\
\text { [162] }\end{array}$ & $\begin{array}{l}\text { Apoe } e^{-1} \text { MyD } 88^{\%} \text { vs. Apoe }{ }^{-/} \\
\text {mice, received HFD. }\end{array}$ & $\begin{array}{l}\downarrow \text { total atherosclerotic lesion area, } \downarrow \text { lipid and macrophage } \\
\text { accumulation in aortic sinus plaques, } \downarrow \text { COX- } 2 \text { in aortic sinus plaques, } \downarrow \\
\text { circulating IL-12 and MCP-1 levels, } \downarrow \text { leukocyte-EC adhesion in Apoe }{ }^{-/} \\
\text {MyD88\% mice. }\end{array}$ & Pro-atherogenic. \\
\hline
\end{tabular}

个, increase; $\downarrow$, decrease; -, no change. Abbreviations: CABG, coronary artery bypass graft surgery; Ldlr, low-density lipoprotein receptor; HFD, high fat diet; i.p., intraperitoneal; Pam3, Pam3CSK4 (TLR2/1 agonist); BM, bone marrow; HCAECs, human coronary artery endothelial cells; MALP-2, macrophage activating lipoprotein-2; P. gingivalis, Porphyromonas gingivalis, SMCs, smooth muscle cells; NF-kB, nuclear factor kB; MCP-1, macrophage chemoattractant protein 1; IL, interleukin; MMP, matrix metalloproteinases; AthSMCs, athesrosclerotic tissue

SMCs; AoSMCs, aorta SMCs; Poly I:C, polyinosinic:polycytidylic acid; LOF, loss-of-function; oxLDL, oxidised low-density lipoprotein; COX-2, cyclooxygenase-2; EC, endothelial cell. 


\subsubsection{TLRs and ACS: the role of TLRs in myocardial ischaemia/reperfusion injury.}

Aside from their role in atherosclerotic development, TLRs are known to be important during an ACS. Patients with ACS experience two waves of inflammation during their event: the initial insult as a result of plaque rupture/erosion and thrombotic occlusion, and the secondary insult as a result of reperfusion of the culprit coronary arteries. The damage caused by the initial myocardial ischaemia is exacerbated upon restoration of blood flow in these arteries and reperfusion of the myocardium. The sudden restoration of blood flow during reperfusion causes a second wave of inflammation, the hallmark of which is the production of ROS $[176,177]$. This can cause further myocardial ischaemia, and this damage is termed myocardial ischaemia/reperfusion (I/R) injury. As such, although reperfusion by thrombolytic therapy or $\mathrm{PCl}$ is necessary to recover maximal myocardial function, these therapies themselves can result in an increased ischaemic region.

It is likely that the wave of inflammation upon reperfusion is mediated, in part, by the release of DAMPs by the damaged myocardium and the subsequent activation of TLRs. As such, there has been emerging evidence to suggest that TLR2 and 4, in particular, have roles in enhancing myocardial I/R injury. Shishido et al. have shown the importance of TLR2 in mediating ventricular remodelling following infarction by demonstrating a reduction in myocardial fibrosis and left ventricular dimensions 4 weeks after AMI in TLR2 knockout mice, compared to wildtype controls [178]. Furthermore, contractile dysfunction was more evident in reperfused hearts from wildtype mice, compared to hearts from TLR2-knockout mice [179]. After reperfusion, endothelial dysfunction was improved in TLR2-deficient mice, compared to wild-type mice [180] indicating TLR2-induced endothelial dysfunction following myocardial I/R injury. Favre et al. also reported a reduction in infarct size and leukocyte infiltration into the myocardium in the absence of TLR2 [180].

These findings are also echoed by Oyama et al. [181] and Chong et al. [182] in TLR4-deficient mice. In these studies, attenuated neutrophil infiltration [181] and attenuated cytokine and chemokine 
production, such as MCP-1 and IL-6 [182], in the reperfused ischaemic myocardium was noted in TLR4-deficient, compared to wildtype, mice. Treatment of mice with eritoran, a TLR4 antagonist, before ischaemia and reperfusion was shown to reduce infarct size and cytokine expression compared to untreated mice [183]. These results reflect the phenotype that is achieved in TLR4 knockout mice.

Taken together, these studies demonstrate the importance of global TLR2 and 4 expression in myocardial I/R injury, and hint at the importance of TLR expression in modulating inflammation during reperfusion. 


\subsection{Platelet-TLR expression and activation.}

TLRs have established roles in exacerbating CAD in response to both infectious (PAMPs) and injurious (DAMPs) mediators. This section discusses the repertoire of platelet-TLRs and the current understanding of platelet responses to TLR agonism. Based on this evidence, we suggest that platelets are able to respond to both infectious and injurious mediators that signal through particular TLRs.

Of the TLRs known to be expressed on and within immune cells, a subset have been well-studied in platelets. The expression and activation of TLR1, 2, 4, and to a lesser extent TLRs 6 and 9, have been characterized in healthy human and murine platelets to varying degrees. As described earlier, this subset of platelet-TLRs were examined in this thesis. This section provides an overview of what is currently known about these platelet-TLRs, of which the expression data (Table 1.5) and activation data (Table 1.6) are summarized in tables below this section. Although platelet-TLRs are variably studied in healthy humans, there remains a lack of understanding of the significance of platelet-TLR expression and activation in disease states such as AMI, where platelets are known to play a large and detrimental role.

\subsubsection{Platelet-TLR1, 2 and 6.}

\subsubsection{Expression.}

Consistently low, but significant, expression of TLR1 has been reported on the surface of platelets previously [184, 185]. Furthermore, platelet-TLR1 mRNA was detected in a large cohort of patients from the Framingham Heart Study [186] and this has been confirmed by others [185]. In this study, platelet-TLR1 mRNA was present in about $50 \%$ of the Framingham participants. However, others recorded negligible TLR1 expression both on the platelet surface and intracellularly by flow cytometry [187]. 
In previous flow cytometry [187, 188] and immunoprecipitation [184] experiments, low plateletsurface TLR2 expression was detected, with approximately $5 \%$ to $15 \%$ of the platelet population expressing platelet-surface TLR2. TLR2 expression has been shown to increase following platelet activation [187], although this response was not seen by others [188]. Intracellular TLR2 expression is moderate, with approximately $15 \%$ of the platelet population expressing intracellular TLR2, and expression increases upon activation [187].

There is little evidence regarding platelet-TLR6 expression; low cell-surface expression of TLR6 has been reported by some [184, 185] but not by others [187].

\subsubsection{Activation.}

In healthy subjects, dose-dependent increases in platelet activation [189] and aggregation [189, 190], VWF release [189] and increased adhesion to a collagen surface [190] have been reported following stimulation with the prototypical TLR2/1 agonist, Pam3CSK4. These results demonstrate the ability of platelets to form a platelet plug in response to TLR2/1 engagement. Furthermore, PF4 [190], thrombospondin and Factor XIIIA [190] are released from platelets upon stimulation with Pam3CSK4. The release of these pro-thrombotic molecules demonstrates an increase in the platelet thrombotic response to TLR2/1 stimulation.

Aside from these pro-thrombotic responses, engaging the TLR2/1 complex induces a range of platelet-immune responses. Pam3CSK4 stimulation significantly increases platelet-monocyte [184] and platelet-neutrophil [184, 189] interactions over very short periods of incubation (10 minutes). Interestingly, attachment of platelets to leukocytes was more abundant following Pam3CSK4 stimulation, when compared to thrombin stimulation [190] and the authors theorized that TLR2/1 engagement induces a stable clot formation by primarily utilizing platelet-leukocyte aggregates.

Page / 40 
The platelet-mediated immune response to periodontiopathogens has been well studied and demonstrates that platelets can directly respond to infection via the TLR2 pathway. An in vivo challenge with Porphyromonas gingivalis, a periodontopathogen that signals via TLR2, induces significant platelet-neutrophil aggregation [184] and platelet release of the chemokine, RANTES [191]. Platelet activation in response to this type of infection, and the resulting direct interaction with neutrophils, has been demonstrated to enhance neutrophil-mediated periodontopathogen clearance in a platelet-TLR2-specific manner [192].

There remains a lack of understanding as to whether platelets can become activated in response to TLR2/6 agonism.

\subsubsection{Platelet-TLR2/1 signalling.}

The effects of agonizing platelet-TLR2 has been attributed to activation of several distinct signalling molecules. TLR2/1 engagement directly activates the phosphoinositide-3-kinase (PI3K)/Akt pathway, as evidenced by a dose-dependent increase in phosphorylation of the serine-473 residue of Akt $[184,190]$ and a dose-dependent increase in p85 co-localization with TLR2 [184], where p85 is the regulatory subunit of PI3K. Serine phosphorylation of Akt was significantly induced within only 15 minutes of Pam3CSK4 stimulation and is similar to the pattern seen in thrombin-activated platelets [190]. Additionally, TLR2/1 signalling induces src- and Syk-family tyrosine phosphorylation of Linker for Activation of T cells (LAT), an adaptor protein, and of phospholipase C (PLC)- $\gamma 2$ [193, 194]. Platelet-TLR2/1-mediated activation of the src/Syk/LAT/PLC 2 pathway mimics the signalling of the classical platelet receptor, FCYRIIA [195]. A role for both PI3K and PLC were confirmed, as inhibition of either protein resulted in complete inhibition of bacteria-induced platelet activation [196]. Additionally, signalling through both the $\mathrm{P} 2 \mathrm{X}_{1} \mathrm{ATP}$ receptor and the $\mathrm{P} 2 \mathrm{Y}_{12}$ ADP receptor are required to amplify classical platelet responses to TLR2/1 engagement [194]. A non-genomic role for NF-kB in response to TLR2/1 engagement was also proposed [189]; Pam3CSK4 stimulation induces the degradation of $\mathrm{IkB} \alpha$ and phosphorylation of the NF-kB p65 
subunit. Phosphorylation of extracellular signal-regulated kinases (ERK) 1 and 2 was rapidly induced following Pam3CSK4 stimulation but abated over longer stimulation times [190]. Similarly Pam3CSK4-mediated phosphorylation of p38 MAPK reached $60-70 \%$ of the levels achieved with thrombin stimulation [190].

\subsubsection{Platelet-TLR4.}

\subsubsection{Expression.}

TLR4 is perhaps the most well characterised platelet-TLR. Several studies report high plateletsurface TLR4 expression [187, 188, 197-199] with between 40 to $60 \%$ of the platelet population expressing platelet-surface TLR4. Some report a significant increase in cell-surface TLR4 expression following thrombin stimulation [197], while others report a decrease in expression following activation with thrombin [187] and with LPS [188].

\subsubsection{Activation.}

LPS is the classical TLR4 agonist that elicits strong inflammatory responses in circulating leukocytes but there are conflicting reports concerning LPS-mediated platelet activation. Some [197, 198] describe increased CD62p expression following incubation with $\leq 10 \mu \mathrm{g} / \mathrm{mL}$ LPS but platelet activation under similar conditions was not described by others $[19,189,200]$. In one study, platelet aggregation was not noted in response to LPS stimulation, up to very high concentrations, but LPS was able to enhance platelet aggregation in response to sub-threshold concentrations of thrombin and collagen [198]. Similarly, the absence of aggregation in response to LPS has been reported by others $[19,201]$. Low ATP and CD62p release has been noted in response to LPS stimulation in some studies $[197,198]$, and it is possible that low-level platelet degranulation plays a role in potentiating the aggregation effect in response to other platelet agonists, but LPS alone is not sufficient to induce aggregation. In other studies, platelets exhibit less sensitivity to LPS than 
other leukocytes [19] and have suggested that LPS may also negatively regulate platelet aggregation [202].

LPS was demonstrated to bind to healthy human platelets, a process which is enhanced by thrombin generation [197], and which demonstrates a possible requirement for thrombin to potentiate the LPS effects on platelets. These synergistic platelet effects of thrombin and LPS stimulation may be relevant in a clinical setting where infection coincides with a hypercoagulable state. Histone release from dying cells was shown to increase cell-surface CD62p and phosphatidylserine expression and this was demonstrated to be a TLR4-specific effect, as all parameters decreased following incubation with a TLR4-neutralizing antibody [203]. This provides evidence for an endogenous agonist inducing an activated platelet phenotype.

In conclusion, the platelet response to LPS in healthy subjects has been widely studied but, despite this, there remains a lack of consensus as to whether platelet-TLR4 activation via LPS can cause robust platelet activation.

\subsubsection{The platelet immune response.}

Reports of platelet-mediated immune response to LPS are similarly mixed. Increased cell-surface CD40L expression [197] and soluble CD40L (sCD40L) release [204] and was seen upon LPS stimulation, although this was not noted by others [189]. LPS engagement of platelet-TLR4 may contribute to the elevated systemic SCD40L level that is seen in septic patients [205], as a large portion of circulating $\mathrm{SCD} 40 \mathrm{~L}$ is released from activated platelets. LPS can induce splicing of unprocessed IL-1ß mRNA as well as translation and accumulation of the protein [201], although large variation in IL-1 $\beta$ protein levels were seen between healthy donors. Others have shown that microparticles which are shed from LPS-activated platelets contain mature IL-1 $\beta$ protein [206]. 
LPS-mediated platelet-leukocyte aggregation is also reported inconsistently [19, 189]. Flow cytometric data has demonstrated an increase in platelet-leukocyte aggregation [201, 207] in response to LPS activation. Similarly, LPS-treated platelets actively attached to adherent neutrophils under flow conditions, a response which only occurs at high LPS concentrations [19]. Similarly, a mouse model of endotoxaemia, which is characterized by neutropenia and thrombocytopenia, shows significant co-aggregation of platelets and neutrophils within the liver sinusoids in response to LPS [19]. However, in other studies, LPS stimulation was unable to stimulate platelet-leukocyte aggregation [189]. As with platelet activation, we cannot yet clearly define the immune-mediated responses of platelets in response to LPS stimulation.

\subsubsection{Platelet-TLR4 signalling.}

TLR4 requires a complex to be formed at the receptor to transduce a signal; receptors CD14 and TLR4 interact with MD-2 at the plasma membrane in nucleated immune cells [208] and is engaged in response to LPS bound to LPS-binding protein (LBP). Platelets are known to express TLR4 (see Figure: Evidence for platelet-TLR expression) and MD-2 [198, 209] both of which increase upon thrombin stimulation. However, platelets do not express CD14 [206, 210, 211] and rely on soluble CD14 for potentiating the LPS signal [201, 209]. Furthermore, sCD14-mediated TLR4 signalling has been described for endothelial and epithelial cells [212]. The disparity in the reports of platelet responses to LPS may be due to a lack of SCD14 in washed platelet preparations. Consistent with this, Rivadeneyra and colleagues demonstrated that the presence of serum was required to observe LPS potentiation of thrombin-induced platelet aggregation [189]. Similarly, Brown and colleagues report increased microparticle shedding when washed platelets are supplemented with recombinant CD14 as well as LBP [206]. Zhang and colleagues demonstrated LPS-induced potentiation of platelet aggregation, where the potentiation effect was seen at much lower LPS concentration with the addition of plasma [198]. 
Platelet-TLR4 utilizes both MyD88 and TRIF as immediate TIR-domain containing proteins. Xiong and colleagues demonstrated a severe reduction in platelet-leukocyte aggregates following preincubation of platelets with either MyD88- or TRIF-inhibitory peptides and LPS stimulation [207]. MyD88 was shown to be essential for platelet-TLR4 signalling [213], as LPS-mediated potentiation of platelet aggregation in response to native agonists was not seen in MyD88-deficient mice [198]. Similarly, incubation with an MyD88 inhibitory peptide significantly attenuated the quantity of mature IL-1 $\beta$ secreted by platelets [206]. Further, IKB $\alpha$ and NF-kB p65 proteins were identified in platelets $[213,214]$. Similar to the non-genomic role proposed for NF-KB upon TLR2/1 engagement, LPS stimulation resulted in the degradation of $1 \kappa B \alpha$ and phosphorylation of p65 subunit of NF-KB [189]. Additionally, thrombin-triggered NF-KB activation was significantly enhanced by the addition of LPS, and completely abolished when platelets were pre-incubated with an NF-KB inhibitor. Activation of kinases associated with TLR signalling in nucleated cells was also noted in platelets following LPS stimulation. Phosphorylation of JNK T183 and Y185 residues increased over 2-fold within 5 minutes of LPS activation of washed platelets and remained elevated over a longer period. Similarly, phosphorylation of Akt T308 and S473 residues is rapid and transient following LPS stimulation [206].

Zhang et al. demonstrated activation of the nitric oxide (NO)/cyclic guanosine monophosphate (cGMP)/protein kinase G (PKG) in platelet-TLR4 signalling [198]. LPS stimulation dose-dependently increased platelet cGMP levels, a response that was significantly attenuated in TLR4-deficient mice. To support this, inhibition of NO synthase (NOS) or of PKG abolishes LPS-induced potentiation of platelet aggregation induced by low concentrations of thrombin. 


\subsubsection{Platelet-TLR9.}

\subsubsection{Expression.}

Variable cell-surface TLR9 expression has been reported on the surface of platelets; both low [187, 215] and high [188] expression has been recorded. Furthermore, TLR9 surface expression increases upon thrombin and collagen stimulation $[188,215]$. However, the physiological significance of cell-surface expression is not yet understood as TLR9 acts intracellularly to identify prokaryotic CpG DNA. High intracellular TLR9 expression has been reported [187] and has been demonstrated to localize to electron-dense tubular system-related granular compartments situated around the platelet periphery [215].

\subsubsection{Activation.}

Incubation with CPG oligodeoxynucleotides (ODNs), prototypical TLR9 agonists, resulted in increased cell-surface CD62p expression [215]. CpG ODN sequestration was seen following a short 20-minute stimulation time and this sequestration was encouraged by collagen pre-incubation [215] and CpG stimulation. Platelet activation has also been demonstrated following incubation with 2-( $\omega$-Carboxyalkyl) pyrrole protein adducts (CAPs) [216]. These are free-radical self-altered ligands that are a product of oxidation, a process that is heavily involved in atherosclerotic progression. Immunostaining experiments demonstrated CAP accumulation in atherosclerotic plaques under hyperlipidemic conditions [216]. Incubation with CAPs results in potent integrin activation and CD62p expression in platelets, indicating an increased ability to bind to fibrinogen and other immune cells, respectively. Furthermore, whole blood pre-treated with CpG and perfused across a type I collagen-coated surface produced thrombus formation [215], which demonstrates the potential for this co-stimulation to cause thrombosis. Similarly, using the $\mathrm{FeCl}_{3-}$ carotid artery model, time to complete thrombotic occlusion of the tail significantly shortened with CAP treatment [216]. 


\subsubsection{Platelet-TLR9 signalling.}

Both Thon et al. and Panigrahi et al. demonstrated the necessity of IRAK1 and 4 activation for transducing the CpG-mediated or CAP-mediated TLR9 signal. Abolition of the co-stimulatory effects of $\mathrm{CpG}$ on collagen-primed platelets was noted following pre-incubation with an IRAK-1/4 inhibitor [215]. Stimulation with CAPs alone or CAPs following ADP-priming induced IRAK1 phosphorylation [216]. Panigrahi and colleagues also demonstrated phosphorylation of the Ser473 residue of Akt following CAP stimulation, a response that was blocked following incubation with inhibitors of PI3K/Akt, Src kinase and Syc kinase. Furthermore, Akt1 and 2, but not Akt3, isoforms were shown to be involved in transducing the CAP-induced signal [216]. 
Table 1.5 Evidence for platelet-TLR expression.

\begin{tabular}{|c|c|c|c|}
\hline$\overline{T L R}$ & Authors [reference] & Evidence for expression & Medium used \\
\hline \multirow[t]{3}{*}{1} & $\begin{array}{l}\text { Blair et al., } 2009 \\
\text { [184] }\end{array}$ & Low cell-surface TLR1 expression on resting human platelets. & WPs \\
\hline & $\begin{array}{l}\text { Cognasse et al., } \\
2005 \text { [187] }\end{array}$ & Negligible cell-surface or intracellular TLR1 expression on resting human platelets. & MPCs \\
\hline & $\begin{array}{l}\text { Shiraki et al., } 2004 \\
\text { [185] }\end{array}$ & $\begin{array}{l}\text { Cell-surface TLR1 expression on platelets within the coronary thrombus of AMI patients, as analysed } \\
\text { by immunohistochemistry. } \\
\text { Results confirmed with PCR and WB. }\end{array}$ & $\begin{array}{l}\text { Coronary thrombus } \\
\text { of AMI patients }\end{array}$ \\
\hline \multirow[t]{3}{*}{2} & $\begin{array}{l}\text { Aslam et al., } 2006 \\
\text { [188] }\end{array}$ & $\begin{array}{l}\text { Low-moderate cell-surface TLR2 expression on resting human platelets ( } 15 \% \text { of platelets). } \\
\text { Expression remains unchanged following thrombin }(1 \mathrm{U} / \mathrm{mL}) \text { or LPS }(50 \mu \mathrm{g} / \mathrm{mL}) \text { stimulation. }\end{array}$ & WPS \\
\hline & $\begin{array}{l}\text { Blair et al., } 2009 \\
\text { [184] }\end{array}$ & $\begin{array}{l}\text { Low cell-surface TLR2 expression on resting human platelets. } \\
\text { Expression confirmed by immunoprecipitation. }\end{array}$ & WPs \\
\hline & $\begin{array}{l}\text { Cognasse et al., } \\
2005 \text { [187] }\end{array}$ & $\begin{array}{l}\text { Low-moderate cell-surface (15\% of platelets) and high intracellular (48\%) TLR2 expression on resting } \\
\text { human platelets. } \\
\text { Significant increase in expression following activation. }\end{array}$ & MPCS \\
\hline \multirow[t]{3}{*}{4} & $\begin{array}{l}\text { Aslam et al., } 2006 \\
\text { [188] }\end{array}$ & $\begin{array}{l}\text { Significant cell-surface TLR4 expression on resting human platelets ( } 60 \% \text { of platelets). } \\
\text { Expression remains unchanged after thrombin }(1 \mathrm{U} / \mathrm{mL}) \text { stimulation. } \\
\text { Expression significantly attenuated after LPS }(50 \mu \mathrm{g} / \mathrm{mL}) \text { stimulation (attenuated to } \sim 33 \%) \text {. }\end{array}$ & WPs \\
\hline & $\begin{array}{l}\text { Cognasse et al., } \\
2005 \text { [187] }\end{array}$ & $\begin{array}{l}\text { Significant cell-surface ( } 59 \% \text { of platelets) and intracellular (79\%) TLR4 expression on resting human } \\
\text { platelets. } \\
\text { Significant increase in intracellular expression following activation, but significant decrease in cell- } \\
\text { surface expression following activation. }\end{array}$ & MPCs \\
\hline & $\begin{array}{l}\text { Zhang et al., } 2009 \\
\text { [198] }\end{array}$ & $\begin{array}{l}\text { Cell-surface TLR4 expression on resting human platelets (amount not quantified). } \\
\text { Results confirmed with WB. }\end{array}$ & WPS \\
\hline
\end{tabular}


Table 1.5. Evidence for platelet-TLR expression (continued).

\begin{tabular}{|c|c|c|c|}
\hline$\overline{T L R}$ & Authors [reference] & Evidence for expression & Medium used \\
\hline \multirow[t]{2}{*}{4} & $\begin{array}{l}\text { Stahl et al., } 2006 \\
\text { [197] }\end{array}$ & $\begin{array}{l}\text { Significant cell-surface TLR4 expression on resting human platelets (median, 43\%; range 24-60\%). } \\
\text { Significant increase in expression after thrombin ( } 1 \mathrm{U} / \mathrm{mL} \text { ) stimulation (median, 79\%; range 64-85\%). }\end{array}$ & PRP \\
\hline & $\begin{array}{l}\text { Andonegui et al., } \\
2005 \text { [199] }\end{array}$ & Significant cell-surface expression of TLR4 on resting human platelets ( $37 \%$ of platelets). & WPS \\
\hline \multirow[t]{3}{*}{6} & $\begin{array}{l}\text { Blair et al., } 2009 \\
\text { [184] }\end{array}$ & Low cell-surface TLR6 expression on resting human platelets. & WPs \\
\hline & $\begin{array}{l}\text { Cognasse et al., } \\
2005[187]\end{array}$ & Negligible cell-surface or intracellular TLR6 expression on resting human platelets. & MPCs \\
\hline & $\begin{array}{l}\text { Shiraki et al., } 2004 \\
\text { [185] }\end{array}$ & $\begin{array}{l}\text { Cell-surface TLR6 expression on platelets within the coronary thrombus of AMI patients, as analysed } \\
\text { by immunohistochemistry. Results confirmed with PCR and WB. }\end{array}$ & $\begin{array}{l}\text { Coronary thrombi } \\
\text { from AMI patients }\end{array}$ \\
\hline \multirow[t]{3}{*}{9} & $\begin{array}{l}\text { Aslam et al., } 2006 \\
\text { [188] }\end{array}$ & $\begin{array}{l}\text { Significant cell-surface TLR9 expression on resting human platelets ( } \sim 37 \% \text { of platelets). Expression } \\
\text { remains unchanged after LPS }(50 \mu \mathrm{g} / \mathrm{mL}) \text { stimulation }(\sim 33 \%) \text {. Expression significantly increased after } \\
\text { thrombin }(1 \mathrm{U} / \mathrm{mL}) \text { stimulation. }\end{array}$ & WPS \\
\hline & $\begin{array}{l}\text { Cognasse et al., } \\
2005 \text { [187] }\end{array}$ & $\begin{array}{l}\text { Low cell-surface ( } 10 \% \text { of platelets) and moderate intracellular (34\%) TLR9 expression on resting } \\
\text { human platelets. }\end{array}$ & MPCs \\
\hline & $\begin{array}{l}\text { Thon et al., } 2012 \\
\text { [215] }\end{array}$ & $\begin{array}{l}\text { Low cell-surface expression on resting ( } \sim 3 \%) \text { and moderate expression on thrombin-activated ( } 25 \%) \\
\text { platelets. TLR9 agonism significantly increases TLR9 cell-surface expression. Intracellular expression of } \\
\text { TLR9 in resting human platelets determined with immunofluorescence microscopy (amount not } \\
\text { quantified). }\end{array}$ & WPs \\
\hline
\end{tabular}

Highlighted in bold: type of expression analysed. MPCs, mixes of platelet concentrates; PRP, platelet-rich plasma; PCR, polymerase chain reaction; WB, western blotting; AMI, acute myocardial infarction; $U$, unit; LPS, lipopolysaccharide. 
Table 1.6 Evidence for TLR-mediated platelet activation.

\begin{tabular}{|c|c|c|c|c|}
\hline TLR & $\begin{array}{l}\text { Authors } \\
\text { [reference] }\end{array}$ & Agonist used & $\begin{array}{l}\text { Medium } \\
\text { used }\end{array}$ & Platelet activation results \\
\hline \multirow[t]{3}{*}{$2 / 1$} & $\begin{array}{l}\text { Blair et al., } \\
2009[184]\end{array}$ & $10 \mu \mathrm{g} / \mathrm{mL}$ Pam3CSK4 & hWB & $\begin{array}{l}\text { Increased number of platelet-monocyte, platelet-neutrophil aggregates following } 10 \text { minutes of } \\
\text { stimulation. }\end{array}$ \\
\hline & $\begin{array}{l}\text { Rivadeneyra } \\
\text { et al., } 2014 \\
\text { [189] }\end{array}$ & $\begin{array}{l}\text { Various } \\
\text { concentrations } \\
\text { Pam3CSK4 }\end{array}$ & hWPs & $\begin{array}{l}\text { Increased platelet aggregation dose-dependently }\left(\mathrm{EC}_{50}=5 \pm 0.2 \mu \mathrm{g} / \mathrm{mL}\right) \text { and significant release of } \\
\mathrm{ADP}(10 \mu \mathrm{g} / \mathrm{mL}) \text { after } 10 \text { minutes of stimulation. Significant release of } \mathrm{VWF}(1 \mu \mathrm{g} / \mathrm{mL}) \text {, significant } \\
\text { increase in surface } \mathrm{CD} 40 \mathrm{~L}(10 \mu \mathrm{g} / \mathrm{mL}) \text { and P-selectin }(1 \mu \mathrm{g} / \mathrm{mL}) \text { expression, increased number of } \\
\text { platelet-neutrophil aggregates }(1 \mu \mathrm{g} / \mathrm{mL}) \text { after } 15 \text { minutes of stimulation. }\end{array}$ \\
\hline & $\begin{array}{l}\text { Assinger et } \\
\text { al., } 2012 \\
{[196]}\end{array}$ & $\begin{array}{l}1 \mu \mathrm{g} / \mathrm{mL} \text { and } 10 \\
\mu \mathrm{g} / \mathrm{mL} \text { Pam3CSK4 }\end{array}$ & hPRP & $\begin{array}{l}\text { Increased surface CD } 40 \mathrm{~L} \text { expression after } 20 \text { minutes of stimulation. Increased SCD } 40 \mathrm{~L} \text { release } \\
\text { after } 1 \text { and } 3 \text { hours of stimulation. }\end{array}$ \\
\hline \multirow[t]{4}{*}{4} & $\begin{array}{l}\text { Zhang et al., } \\
2009 \text { [198] }\end{array}$ & $\begin{array}{l}\text { 1-10 } \mu \mathrm{g} / \mathrm{mL} \mathrm{LPS} \\
\text { (serotype 0111:B4) }\end{array}$ & hWPs & $\begin{array}{l}\text { Potentiated aggregation in response to low concentrations of thrombin }(0.0185 \mathrm{U} / \mathrm{mL}) \text { and } \\
\text { collagen }(0.3 \mathrm{\mu g} / \mathrm{mL}) \text { after } 10 \text { minutes of stimulation. Low ATP release with LPS alone after } 10 \\
\text { minutes of stimulation. Increased cell-surface P-selectin expression with LPS alone after } 30 \\
\text { minutes of stimulation. }\end{array}$ \\
\hline & $\begin{array}{l}\text { Xiong et al., } \\
2011 \text { [207] }\end{array}$ & $\begin{array}{l}500 \mathrm{ng} / \mathrm{mL} \mathrm{LPS} \\
(0111: \mathrm{B} 4)\end{array}$ & $\mathrm{mWB}$ & $\begin{array}{l}\text { Shape change, extended pseudopodia, aggregates formed at } 30 \text { and } 60 \text { minutes after stimulation. } \\
\text { Platelet-leukocyte aggregates increased time-dependently from } 5 \text { to } 60 \text { minutes of stimulation. } \\
\text { Number of aggregates attenuated in whole blood incubated with MyD88 or TRIF-inhibitory } \\
\text { peptides prior to } 60 \text { minutes stimulation. }\end{array}$ \\
\hline & $\begin{array}{l}\text { Stahl et al., } \\
2006[197]\end{array}$ & $\begin{array}{l}1 \mu \mathrm{g} / \mathrm{mL} \mathrm{LPS} \text { (various } \\
\text { serotypes) }\end{array}$ & hPRP & $\begin{array}{l}\text { Significant LPS-binding to platelets, significant increase in PAC-1 and P-selectin expression, } \\
\text { significant increase in fibrinogen binding after } 45 \text { minutes of stimulation. }\end{array}$ \\
\hline & $\begin{array}{l}\text { Rivadeneyra } \\
\text { et al., } 2014 \\
\text { [189] }\end{array}$ & $\begin{array}{l}\text { Various } \\
\text { concentrations LPS } \\
\text { (0111:B4) }\end{array}$ & hWPs & $\begin{array}{l}\text { Co-stimulation }(10 \mu \mathrm{g} / \mathrm{mL}) \text { with thrombin }(0.01 \mathrm{U} / \mathrm{mL}) \text { induces release of ATP after } 10 \text { minutes of } \\
\text { stimulation. Increased release of } \mathrm{VWF} \text { with } 1 \mu \mathrm{g} / \mathrm{mL} \text { LPS alone after } 15 \text { minutes of stimulation. No } \\
\text { increase in CD } 40 \mathrm{~L} \text { or P-selectin expression, no increase in platelet-neutrophil aggregates with } 1 \\
\mu \mathrm{g} / \mathrm{mL} \text { LPS alone after } 15 \text { minutes of stimulation. Could not modify thrombin-induced expression } \\
\text { of these parameters. }\end{array}$ \\
\hline
\end{tabular}


Table 1.6. Evidence for TLR mediated platelet activation (continued).

\begin{tabular}{|c|c|c|c|c|}
\hline TLR & $\begin{array}{l}\text { Authors } \\
\text { [reference] }\end{array}$ & Agonist used & $\begin{array}{l}\text { Medium } \\
\text { used }\end{array}$ & Platelet activation results \\
\hline \multirow[t]{4}{*}{4} & $\begin{array}{l}\text { Clark et al., } \\
2007 \text { [19] }\end{array}$ & $\begin{array}{l}5 \mu \mathrm{g} / \mathrm{mL} \text { LPS } \\
(0111: \mathrm{B} 4)\end{array}$ & hWPs & $\begin{array}{l}\text { No increase in cell-surface P-selectin after } 10 \text { minutes of stimulation. No increase in aggregation. } \\
\text { Induced platelet-neutrophil interactions and neutrophil degranulation. }\end{array}$ \\
\hline & $\begin{array}{l}\text { Brown et al., } \\
2011[206]\end{array}$ & $\begin{array}{l}100 \mathrm{ng} / \mathrm{mL} \text { LPS with } \\
100 \mathrm{ng} / \mathrm{mL} \\
\text { recombinant CD14 } \\
\text { and LBP }\end{array}$ & hWPs & 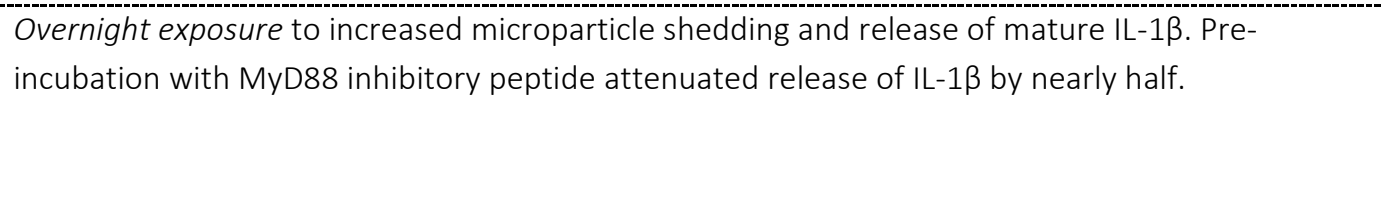 \\
\hline & $\begin{array}{l}\text { Assinger et } \\
\text { al., } 2012 \\
{[196]}\end{array}$ & 1 and $10 \mu \mathrm{g} / \mathrm{mL}$ LPS & hPRP & $\begin{array}{l}\text { Increased cell-surface CD40L expression after } 20 \text { minutes of stimulation. Increased SCD4OL } \\
\text { release after } 1 \text { and } 3 \text { hours of stimulation. }\end{array}$ \\
\hline & $\begin{array}{l}\text { Andonegui et } \\
\text { al., } 2005 \\
\text { [199] }\end{array}$ & $5 \mu \mathrm{g} / \mathrm{mL}$ LPS & mWPs & $\begin{array}{l}\text { Increased adhesion to fibrinogen at low shear rates, } 50 \% \text { as effective as thrombin after } 30 \\
\text { minutes of stimulation. }\end{array}$ \\
\hline \multirow[t]{2}{*}{9} & $\begin{array}{l}\text { Panigrahi et } \\
\text { al., } 2013 \\
{[216]}\end{array}$ & $\begin{array}{l}\text { CAPs synthesized in- } \\
\text { house }(1.5 \mu \mathrm{M} \\
\text { albumin, } 12.5 \mu \mathrm{M} \\
\text { pyrrole) }\end{array}$ & hWPs & $\begin{array}{l}\text { Increased P-selectin and PAC-1 expression after } 20 \text { minutes of stimulation. Increased modification } \\
\text { of CAPs increases platelet aggregation, low modification CAPs can induce aggregation in the } \\
\text { presence of TRAP or ADP. }\end{array}$ \\
\hline & $\begin{array}{l}\text { Thon et al., } \\
2012[215]\end{array}$ & $5 \mu \mathrm{M}$ ODN2006 & hWPs & $\begin{array}{l}\text { Increased cell-surface P-selectin expression and increased CpG sequestration after } 20 \text { minutes of } \\
\text { stimulation. }\end{array}$ \\
\hline
\end{tabular}

Highlighted in bold: methods of measuring platelet activation. Highlighted in italics: time of stimulation. Abbreviations: h, human; m, murine; WB, whole blood; WPs, washed platelets; ADP, adenosine triphosphate; CD40L, CD40 ligand; VWF, von Willebrand factor; IL-1 $\beta$, interleukin-1B; PF4, platelet factor 4; MyD88, myeloid differentiation primary response protein 88; TRIF, Toll-IL-1 receptor (TIR)-domain containing adapter-inducing interferon $\beta$; LPS, lipopolysaccharide; LBP, LPS-binding protein; TRAP, thrombin receptor activating peptide; CAPs, 2 ( $\omega$ Carboxyalkyl)pyrrole protein adducts; $\mathrm{CpG}$, cytosine-phosphate-guanine. 


\subsection{The link between infection and CAD.}

The association between systemic inflammation and atherosclerotic development suggests that an inducer of inflammation such as an infection, even if sub-clinical, may play a role in triggering AMI [217].

\subsubsection{Atherosclerosis and infection.}

As an example, severe intimal infiltration of inflammatory and smooth muscle cells were observed in atherosclerotic plaques in $\mathrm{ApoE}^{-/-}$mice following infection with influenza $\mathrm{A}$ virus, a response that was not observed in infected wild-type mice [218]. At autopsy, pathological examination of coronary atherosclerotic sections found significant increases in leukocyte infiltration into plaques in patients with infection compared to those without infection [219]. C. pneumoniae infection of atherogenic mice reduces the fibrous cap of plaques and increases plaque instability [220], and infection promotes plaque-localized TNF- $\alpha$ and IFN- $\gamma$ production in the aorta of ApoE ${ }^{-/-}$mice [221]. There are several other examples where infection has been shown to trigger inflammation and atherosclerotic development [222-225].

\subsubsection{AMl and infection.}

Well-designed retrospective studies have demonstrated a consistent two- to three-fold increase in the risk of AMI within 1-2 weeks following infection, with the peak incidence of AMI occurring within the first few days of infection onset. Additionally, a number of predominantly case-control studies, ranging from small cohort sizes (40 cases, 71 controls [226]) to large cohort sizes (11 155 cases, 11155 controls [227]), have demonstrated an association of acute infection and the incidence of AMI, with an odds ratio of between 2.2 and 4.2 [226-231]. 
During influenza epidemics, increased morbidity and mortality related to AMI was noted [232], and the authors urged public health organizations to incorporate cardiovascular treatment into preparations for influenza pandemics. A study that examined early influenza pandemics (19181929) in the United States concluded that cardiovascular events was the leading cause of excess mortality behind deaths caused by influenza and pneumonia [233]. Upon examination of a large number of autopsies, AMI causing death was 30\% more likely to happen during the influenza season when compared to the off-season [234]. In a retrospective study of patients admitted for pneumococcal pneumonia and followed up for 5 years, approximately $20 \%$ of patients had at least one major cardiovascular event [235], a finding that was confirmed by others [236, 237]. Patients with a respiratory tract infection [230] or influenza-like symptoms [226] are 3 times as likely to experience an $\mathrm{AMI}$ as patients without these symptoms. Failure to respond to pneumonia treatment in severe cases of community acquired pneumonia was associated with an increased risk of experiencing an AMI [237]. These studies indicate an association between infection and the onset of AMI.

\subsubsection{Seasonal variation in AMI.}

It has been consistently demonstrated that there is a seasonal pattern of AMI incidence and death, with a peak in winter. In examining STEMI patients admitted to Waikato hospital, New Zealand, between 1998 and 2007, 35 STEMI cases per month were admitted to hospital during the winter months, and this was higher than the rate reported for summer (27 STEMI cases per month) [238]. Elsewhere, an $11 \%$ increase in the risk of AMI has been reported in winter versus non-winter months [239]. A relative risk of 1.052 for the incidence of AMI deaths was recorded during winter and this is higher than the relative risk of 0.954 recorded during summer [240]. Similarly, a rate of 4.8 deaths due to AMI per 10,000 inhabitants was reported for winter and was statistically higher than the rate of 3.5 deaths due to AMI per 10,000 [241]. 


\subsection{Overall aims.}

We suggest that the TLR pathways, which are not currently therapeutically targeted, may represent an alternative pathway of platelet activation in AMI despite treatment with DAPT. This thesis explores platelet-TLR expression and TLR-mediated platelet activation in healthy subjects and subjects with AMI, the effect of DAPT on this type of activation, and the effect of platelets on leukocyte function in response to TLR stimulation. The overall hypotheses and aims of this thesis are as follows:

Firstly, we hypothesized that there will be an increase in platelet-TLR expression in AMI subjects, compared to healthy subjects. We have tested this hypothesis in Chapter 2 and Chapter 3, with the following aims:

1. To examine baseline TLR9 expression in healthy subjects and in AMI subjects treated with DAPT, and to determine how TLR9 expression changed in response to platelet activation in both cohorts (Chapter 2).

2. To examine whether total protein expression of platelet-TLR 1, 2, 4 and 6 changed between healthy subjects and AMI subjects treated with DAPT (Chapter 3).

Secondly, we hypothesized that the TLR pathways are a mechanism by which platelets can become activated. We have tested this in Chapter 2 and Chapter 3:

3. To examine platelet activation in response to ODN2006, a TLR9 agonist, in healthy subjects and in AMI subjects treated with DAPT (Chapter 2).

4. To compare the level of platelet activation in response to TLR4, TLR2/1 and TLR2/6 agonism in healthy subjects and in AMI subjects treated with DAPT (Chapter 3). 
We also hypothesized that anti-platelet therapy does not inhibit, or only slightly inhibits platelet activation in response to direct TLR stimulation. We have tested this in Chapter 4:

5. To determine the extent to which aspirin alone or in combination with ticagrelor, a potent $P 2 Y_{12}$ receptor antagonist, can inhibit platelet activation in response to TLR2/1, TLR4 and TLR9 stimulation.

Fourthly, we hypothesized that platelets will modify the responses of leukocyte subsets in a TLR agonist-specific manner. We have tested this in Chapter 5:

6. To determine the extent to which platelets modulate PBMC (monocyte, $T$ cell) and granulocyte (neutrophil) responses in vitro to TLR4, TLR2/1, and TLR2/6 agonism in healthy subjects.

Lastly, on the basis of our previous results from Chapter 5, we hypothesized that platelets will attenuate various neutrophil functions in a TLR agonist-specific manner. This was examined in Chapter 6:

7. To determine the extent to which platelets can modulate multiple markers of neutrophil function in response to in vitro stimulation with $T L R 4, T L R 2 / 1$, and TLR2/6 agonists in healthy subjects. 


\section{Chapter 2. TLR9 expression and ODN2006-mediated platelet activation in AMI patients on DAPT.}

Publication arising from this chapter:

Hally, K. E., La Flamme, A. C., Larsen, P. D., \& Harding, S. A. (2016). Toll-like receptor 9 expression and activation in acute coronary syndrome patients on dual anti-platelet therapy. Thrombosis Research, 148, 89-95. doi:http://dx.doi.org/10.1016/j.thromres.2016.10.026. 


\subsection{Introduction.}

As we have established in Chapter 1, platelet activation can occur via multiple thrombotic and inflammatory signalling pathways. Although AMI patients are treated with DAPT, a proportion of patients will continue to experience recurrent ischaemic events despite treatment with DAPT [242, 243]. Alternative activation pathways that are not currently therapeutically targeted may contribute to this ongoing ischaemic risk in some patients. Platelet-TLRs may represent such alternative pathways. A subset of TLRs are known to be expressed on and within platelets [185, 187, 188] and various immune-driven, anti-microbial effects have been attributed to platelet-TLR agonism [9, 244, 245].

The subset of platelet-TLRs (TLR1, 2, 4, 6 and 9) that was examined in this thesis can neatly be divided into two groups based on their sub-cellular localization. Platelet-TLR9 is predominantly expressed intracellularly and, therefore, recognizes microbial nucleic acids. Platelet-TLR1, 2, 4 and 6 are predominantly expressed on the platelet surface and respond to different extracellular lipid molecular patterns. Characterization of platelet-TLR expression and TLR-mediated platelet activation has been examined in Chapters 2 and 3 according to their sub-cellular localization; platelet-TLR9 is addressed in Chapter 2 and platelet-TLR1, 2, 4 and 6 are addressed in Chapter 3.

TLR9 traditionally recognizes unmenthylated CpG ODNs present in bacterial and viral DNA [246]. More recently, platelet-TLR9 has been shown to respond to free radical-altered self-ligands, thereby acting as a sensor of endogenous 'danger' [216]. Stimulation with these free radicalaltered ligands or with the prototypical TLR9 agonist, ODN2006, has been shown to activate platelets from healthy subjects previously $[215,216]$. However, TLR9 expression and platelet activation in response to a TLR9 agonist remains incompletely characterized in healthy subjects, and there is no data examining its role in pathological conditions such as AMI. 
We hypothesize that platelet-TLR9 expression will increase with platelet activation and will increase during AMI. We further hypothesize that platelets will become activated in response to a TLR9 agonist in both a cohort of healthy subjects and a cohort of AMI subjects treated with DAPT. Therefore, we addressed the following aims in this study:

1. To examine baseline TLR9 expression in healthy subjects and in AMI subjects treated with DAPT, and to determine how TLR9 expression changed in response to platelet activation in both cohorts.

2. To examine platelet activation in response to ODN2006, a TLR9 agonist, in both cohorts. 


\subsection{Materials and methods.}

\subsubsection{Subject recruitment.}

\subsubsection{Healthy subjects.}

Two distinct healthy cohorts were recruited for this study: a cohort of 5 healthy subjects was recruited to examine TLR9 expression in platelets and a separate cohort of 16 healthy subjects was recruited to examine platelet activation in response to ODN2006. Healthy subjects who had no known cardiovascular disease, diabetes mellitus or any inflammatory or immunological disease were recruited. Exclusion criteria included an acute illness within 6 weeks prior to recruitment, pregnancy, a known platelet function disorder, a platelet count of less than $100 \times 10^{9} / \mathrm{L}$ or if they had taken anti-platelet agents, non-steroidal anti-inflammatory drugs or any cardiovascular or immune-modulating medication within 7 days prior to recruitment. The healthy subject component of the study was reviewed and approved by the University of Otago Human Ethics Committee, New Zealand (HE16/004), and all subjects provided written informed consent.

\subsubsection{AMI subjects.}

As defined for healthy subjects, a cohort of 5 AMI subjects was recruited to examine platelet-TLR9 expression and a cohort of 16 AMI subjects was recruited to examine ODN2006-mediated platelet activation. AMI subjects who were adequately treated with DAPT were recruited prior to coronary angiography. AMI was defined according to the Third Universal Definition of Myocardial Infarction [247]. AMI subjects were recruited if they had a dynamic elevation in cardiac troponin levels with either symptoms suggestive of myocardial ischemia and/or $\geq 1 \mathrm{~mm}$ of new ST segment deviation or T wave inversion on an ECG in at least 2 contiguous leads. All AMI subjects were categorized as presenting with either NSTEMI or STEMI. Adequate DAPT was defined as chronic therapy of $\geq 5$ days of aspirin ( $\geq 75 \mathrm{mg}$ once daily) with either clopidogrel ( $\geq 75 \mathrm{mg}$ once daily) or ticagrelor ( $\geq 90$ mg twice daily) and/or loading with $\geq 300 \mathrm{mg}$ aspirin at least 2 hours with either $\geq 300 \mathrm{mg}$ 
clopidogrel at least 6 hours or $\geq 180 \mathrm{mg}$ ticagrelor at least 2 hours prior to recruitment. Patients were excluded if they were administered with a fibrinolytic agent within 24 hours or a glycoprotein IIb/IIla receptor antagonist within a week prior to recruitment, had a concurrent illness, a known platelet function disorder and/or a platelet count of less than $100 \times 10^{9} / \mathrm{L}$. All enrolled AMI subjects underwent coronary angiography and, following this, either underwent $\mathrm{PCl}$, were referred for CABG or were medically managed. The AMI subject component of the study was reviewed and approved by the Health and Disability Ethics Committee, New Zealand (16/CEN/68) and all subjects provided written informed consent.

For all subjects, demographics, relevant clinical characteristics and current medications were obtained following consent.

\subsubsection{Platelet preparation.}

Blood was drawn via venepuncture from healthy subjects into hirudin anticoagulated tubes (Dynabyte, Munich, Germany). For AMI subjects, blood was sampled via intra-arterial sheath immediately after insertion and prior to heparin administration in the cardiac catheterization laboratory. Platelet rich plasma (PRP) was prepared by centrifuging whole blood (WB) at $200 \times \mathrm{xg}$ for 12 minutes. PRP was centrifuged at $100 \mathrm{xg}$ for 20 minutes to pellet contaminating leukocytes. Leukocyte-depleted PRP was adjusted to 100,000/ $\mu \mathrm{L}$ with phosphate-buffered saline (PBS; 145 $\mathrm{mM} \mathrm{NaCl}$, 8.7 mM Na $2 \mathrm{HPO}_{4}, 1.3 \mathrm{mM} \mathrm{NaH}_{2} \mathrm{PO}_{4}$; also see Appendix 2).

\subsubsection{Flow cytometric analysis of TLR9 expression.}

The flow cytometry panel design for TLR9 detection is describe in Table 2.1. PRP was incubated with $1 \mu \mathrm{M}$ or $10 \mu \mathrm{M}$ TRAP6 (Tocris Bioscience, Bristol, UK) for 15 minutes, or left unstimulated for 15 minutes, at room temperature. $1 \mu \mathrm{M}$ and $10 \mu \mathrm{M}$ TRAP stimulation produces low and maximal platelet activation, respectively. Following this incubation, PRP was incubated with anti-CD42a- 
PerCP (clone Beb-1; Becton Dickinson, San Jose, CA, USA) for 20 minutes at room temperature. Separately, an aliquot of PRP was incubated with anti-CD62p-PE (clone AK4; BioLegend, San Diego, CA, USA), a platelet activation marker, to ensure sufficient platelet activation was being achieved under these stimulation conditions. In parallel, unstimulated and stimulated PRP was incubated with PerCP-IgG1,k (Becton Dickinson) or PE-IgG1,k (BioLegend) isotype controls for 20 minutes at room temperature. All samples were fixed with $1 \mathrm{~mL}$ 1\% paraformaldehyde, centrifuged at 1000 xg for 20 minutes at room temperature, permeabilized and pelleted twice in $1 \mathrm{~mL}$ permeabilization wash buffer (BioLegend). Resuspended in residual permeabilization wash buffer, platelets were incubated with anti-TLR9-FITC (clone 26C593.2; Thermo Fisher Scientific, Waltham, MA, USA) in staining buffer ( $2 \%$ fetal calf serum, $0.1 \%$ sodium azide in phosphate-buffered saline) for 20 minutes in the dark at room temperature. Following staining, platelets were diluted in $1 \mathrm{~mL}$ permeabilization wash buffer, pelleted and finally resuspended in $250 \mu \mathrm{L}$ staining buffer for analysis. For TLR9 expression, the geometric mean fluorescence intensity (MFI) was determined. Flow cytometric data was analysed using FlowJo software (Tree star, Ashland, OR, USA).

Flow cytometric detection of monocyte-TLR9 was used as a positive control. WB was incubated with either anti-CD64-APC/Cy7 (clone 10.1; BioLegend) or APC/Cy7-IgG1,k isotype control (BioLegend) for 20 minutes at room temperature before fixation and incubation in red blood cell Iysis buffer (Qiagen, Venlo, Netherlands) for 15 minutes at room temperature. Lysed WB was centrifuged at $100 \times \mathrm{g}$ for 20 minutes at room temperature prior to permeabilization and staining with anti-TLR9-FITC under the same conditions as described for PRP. All samples were analysed on a Millipore Guava easyCyte 8HT flow cytometer (Merck Millipore, Darmstadt, Germany).

The platelet and monocyte population were identified as described in Figure 2.1. For TLR9 expression, the MFI was determined. Representative examples of TLR9 expression in the monocyte and platelet population are given in Figure 2.2. All flow cytometric data was analysed using FlowJo software (Tree star, Ashland, OR, USA). 
Table 2.1 Panel design for TLR9 expression in platelets and monocytes.

\begin{tabular}{|c|l|l|l|}
\hline & $\begin{array}{l}\text { Laser/channel } \\
\text { (fluorophore) }\end{array}$ & $\begin{array}{l}\text { Antibody target } \\
\text { (clone) }\end{array}$ & $\begin{array}{l}\text { Concentration in final staining } \\
\text { volume (final dilution) }\end{array}$ \\
\hline $\begin{array}{c}\text { Platelet-TLR } \\
\text { expression }\end{array}$ & Blue/Red (PerCP) & CD42a (Beb1) & $1.25 \mu \mathrm{g} / \mathrm{mL}(1 \mathrm{in} \mathrm{10)}$ \\
\hline $\begin{array}{c}\text { Monocyte-TLR } \\
\text { expression }\end{array}$ & Red/NIR2 (APC/Cy7) & CD64 (10.1) & $1.00 \mu \mathrm{g} / \mathrm{mL}(1 \mathrm{in} \mathrm{200)}$ \\
\hline Blue/Green (FITC) & TLR9 (26C593.2) & $40.00 \mu \mathrm{gg} / \mathrm{mL}(1 \mathrm{in} 12.5)$ \\
\hline
\end{tabular}

A list of all antibody formats, clones, isotypes and manufacturers can be found in Appendix 1. 


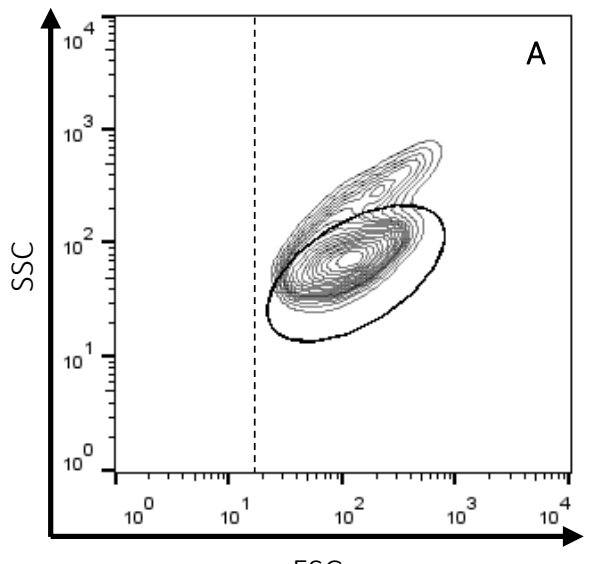

FSC
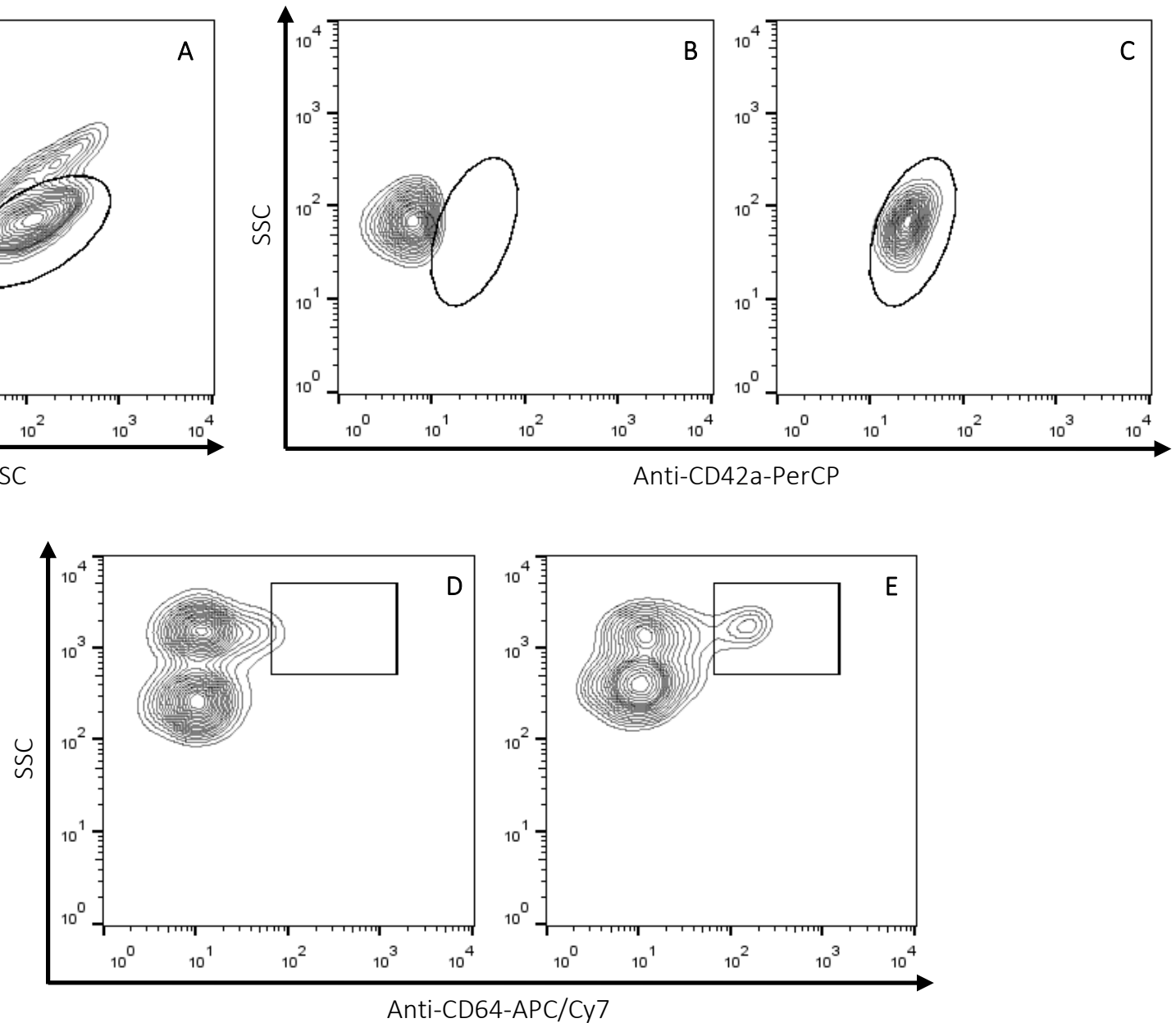

Figure 2.1 The platelet and monocyte populations were identified by flow cytometry.

The platelet population was identified from PRP by their forward and side scatter properties (A) and, further, by their CD42a-positivity (C) against the isotype control (B). The gates in (B) and (C) stem from the gate in (A). The monocyte population was identified from lysed WB by their CD64 positivity (E) against the isotype control (D). 

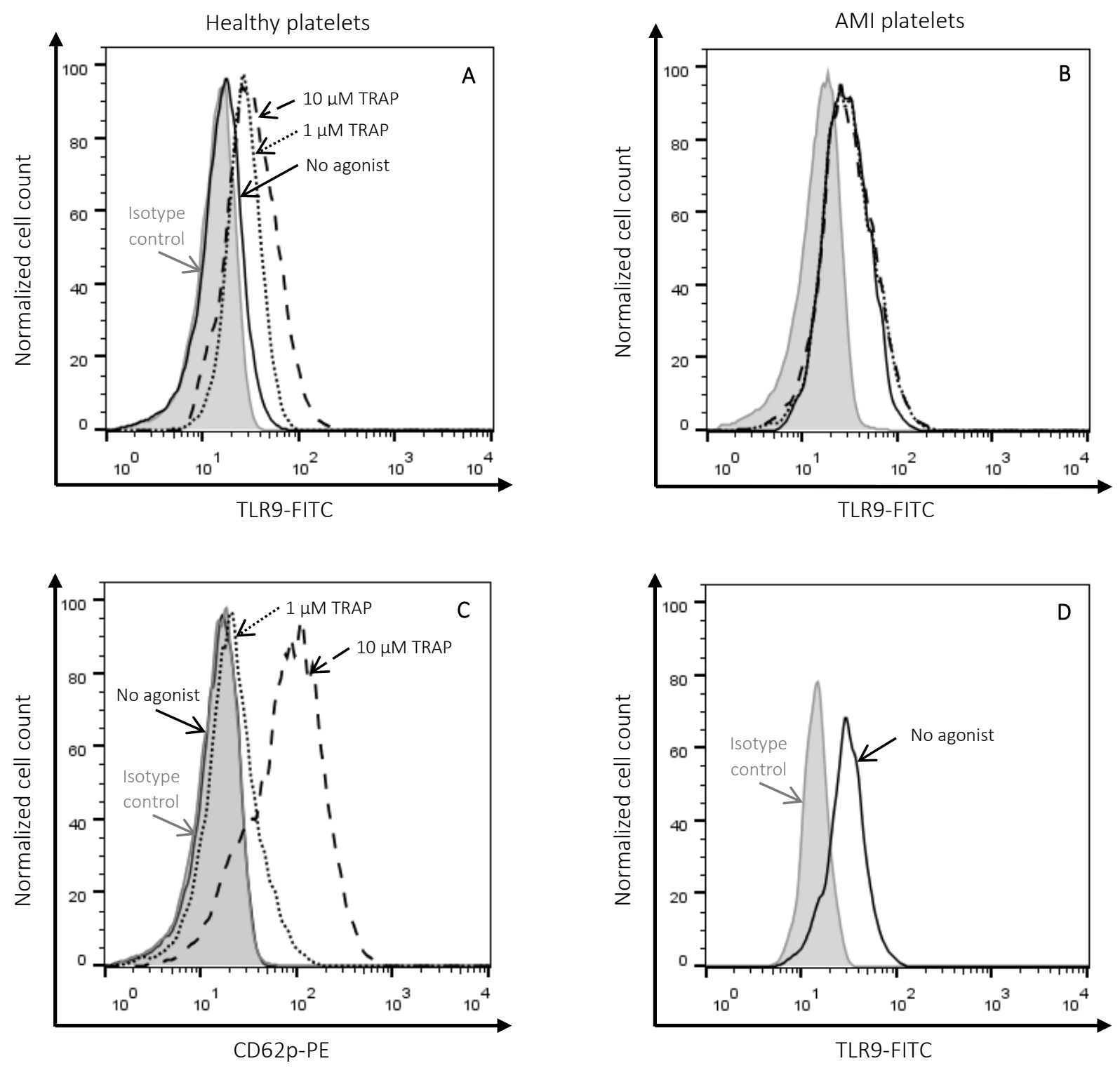

Figure 2.2 TLR9 expression in healthy and AMI platelets was assessed by flow cytometry.

Representative plots of TLR9 expression in healthy (A) and in AMI (B) platelets under resting and activated conditions are shown. Adequate platelet activation was achieved under each of the TRAP stimulation conditions, as measured by cell-surface CD62p expression, which indicates an activated platelet (C). TLR9 expression in resting monocytes was used as a positive control (D). Filled grey line, isotype control; solid black line, no agonist; dotted black line, $1 \mu \mathrm{M}$ TRAP; dashed line, $10 \mu \mathrm{M}$ TRAP. 


\subsubsection{Western blot analysis of TLR9 expression.}

For whole platelet lysate preparation, PRP was either stimulated with $1 \mu \mathrm{M}$ or $10 \mu \mathrm{M}$ TRAP6 or left unstimulated for 50 minutes at RT. Prostaglandin E1 was added to a final concentration $1 \mu \mathrm{M}$ prior to centrifugation at $1,000 \times \mathrm{g}$ for 20 minutes at RT. The platelet pellet was resuspended in radio-immunoprecipitation buffer (10 mM Tris- $\mathrm{HCl}, 150$ mM NaCl, 1 mM EDTA, 1\% Triton-X-100, $0.1 \%$ SDS, $1 \%$ sodium deoxycholate, $\mathrm{pH} 7.5$; also see Appendix 2) containing $10 \mu \mathrm{L} / \mathrm{mL}$ protease inhibitor cocktail (Sigma Aldrich, St. Louis, MO, USA) and incubated for 60 minutes at $4^{\circ} \mathrm{C}$. Cell debris was removed by centrifugation at 14,000 x g for 5 minutes. The protein concentration of whole cell lysates was quantified using the bicinchoninic acid assay (BCA; Thermo Fisher). Aliquots were stored at $-20^{\circ} \mathrm{C}$ until analysis. For western blotting analysis, each cell lysate was incubated in Laemmli buffer (30 mM Tris-HCl, 2\% SDS, 20\% glycerol, 1\% bromophenol blue) containing 10\% final concentration of $\beta$-mercaptoethanol and boiled at $95^{\circ} \mathrm{C}$ for 5 minutes. $50 \mu$ g platelet lysate was loaded onto an 8\% SDS-PAGE and run at $120 \mathrm{~V}$ for 120 minutes at room temperature in SDSPAGE running buffer (25 mM Tris, $192 \mathrm{mM}$ glycine, 1\% SDS). The proteins were blotted onto a PVDF membrane at $20 \mathrm{~V}$ for 17 hours at $4^{\circ} \mathrm{C}$ in transfer buffer (25 mM Tris, $192 \mathrm{mM}$ glycine, 10\% methanol) in a wet transfer system. The membrane was blocked with 5\% BSA in Tris-buffered saline (TBS; $50 \mathrm{mM}$ Tris, $150 \mathrm{mM} \mathrm{NaCl}$, pH 7.4) with 0.1\% Tween 20 (TBS-T) for 1 hour at room temperature and probed with primary mouse anti-human TLR9 (clone 26C593.2; Thermo Fisher Scientific) overnight at $4^{\circ} \mathrm{C}$. After washing with TBS-T, the membrane was incubated with a secondary goat anti-mouse antibody conjugated to horse radish peroxidase (HRP; Bio Rad, Hercules, CA, USA) for 1 hour at room temperature. Antibody-antigen complexes were then detected using an enhanced-chemiluminescent detection system (Bio Rad). The blots were then

stripped as per manufacturer's instructions (Restore Stripping Buffer, Thermo Fisher Scientific) and probed with primary mouse anti-human $\beta$-actin (clone AC-74; Sigma Aldrich) for 3 hours at room temperature before incubation with HRP conjugated secondary goat anti-mouse antibody and detection using enhanced-chemiluminescence detection. 


\subsubsection{Flow cytometric detection of ODN2006-mediated platelet activation.}

The flow cytometry panel design for ODN2006-mediated platelet activation is described in Table 2.2. Platelet activation in response to ODN2006 was assessed in both WB and PRP. Platelet activation in PRP determines the component of activation that is mediated by direct TLR agonism. Incubation in WB determines whether any component of platelet activation is mediated by indirect TLR agonism through 'off-platelet' TLR pathways. $2 \mu \mathrm{L}$ PRP or WB was incubated with 5 $\mu \mathrm{M}$ of the TLR9 agonist, ODN2006 (InvivoGen, San Diego, CA, USA). As a positive control, PRP and WB were incubated with TRAP6 to a final concentration of $2.5 \mu \mathrm{M}$ (sub-maximal platelet activation) and $10 \mu \mathrm{M}$ (maximal platelet activation). As a negative control, PRP and WB were incubated with PBS. All samples were incubated for 15 minutes at room temperature. Following stimulation, each aliquot of PRP and WB was incubated with anti-CD42a-PerCP (clone Beb-1; Becton Dickinson), anti-CD62p-PE (clone AK4; BioLegend), and anti-CD63-AF647 (clone H5C6; BioLegend) in a $50 \mu \mathrm{L}$ total staining volume for 20 minutes in the dark at room temperature. Cellsurface CD62p and CD63 expression indicates an activated platelet. These are markers of platelet degranulation; CD62p is released from the alpha granules and CD63 is released from the dense granules, and both markers are expressed on the surface of platelets upon platelet activation. In parallel, aliquots of PRP and WB were incubated with PerCP-mouse IgG1,K (Becton Dickinson), PEmouse IgG1,k (BioLegend) or AF647-mouse IgG1,k (BioLegend) isotype controls. All samples were fixed with $1 \mathrm{~mL}$ 1\% paraformaldehyde following stimulation and staining and analysed by flow cytometry. Platelets were identified in PRP and WB as shown in Figure 2.3. For CD62p expression, where a distinct positive and negative platelet population was observed, the percentage of CD42apositive platelets that were positive for this activation marker was determined. For CD63 expression, where a fluorescence shift of the whole platelet population was observed, the MFI was determined. Representative plots of CD62p and CD63 expression in platelets are shown in Figure 2.3. 
Table 2.2 Panel design for ODN2006-mediated platelet activation.

\begin{tabular}{|c|l|l|l|}
\hline & $\begin{array}{l}\text { Channel } \\
\text { (fluorophore) }\end{array}$ & $\begin{array}{l}\text { Antibody target } \\
\text { (clone) }\end{array}$ & $\begin{array}{l}\text { Concentration in final staining } \\
\text { volume (final dilution) }\end{array}$ \\
\hline $\begin{array}{c}\text { ODN2006-mediated } \\
\text { activation }\end{array}$ & Blue/Red (PerCP) & CD42a (Beb1) & $1.25 \mu \mathrm{g} / \mathrm{mL}(1: 10)$ \\
& Blue/Yellow (PE) & CD62p (AK-4) & $1 \mu \mathrm{gg} / \mathrm{mL}(1: 100)$ \\
& Red/Red2 (AF647) & CD63 (H5C6) & $2 \mu \mathrm{mL}(1: 100)$ \\
\hline
\end{tabular}

A list of all antibody formats, clones, isotypes and manufacturers can be found in Appendix 1. 

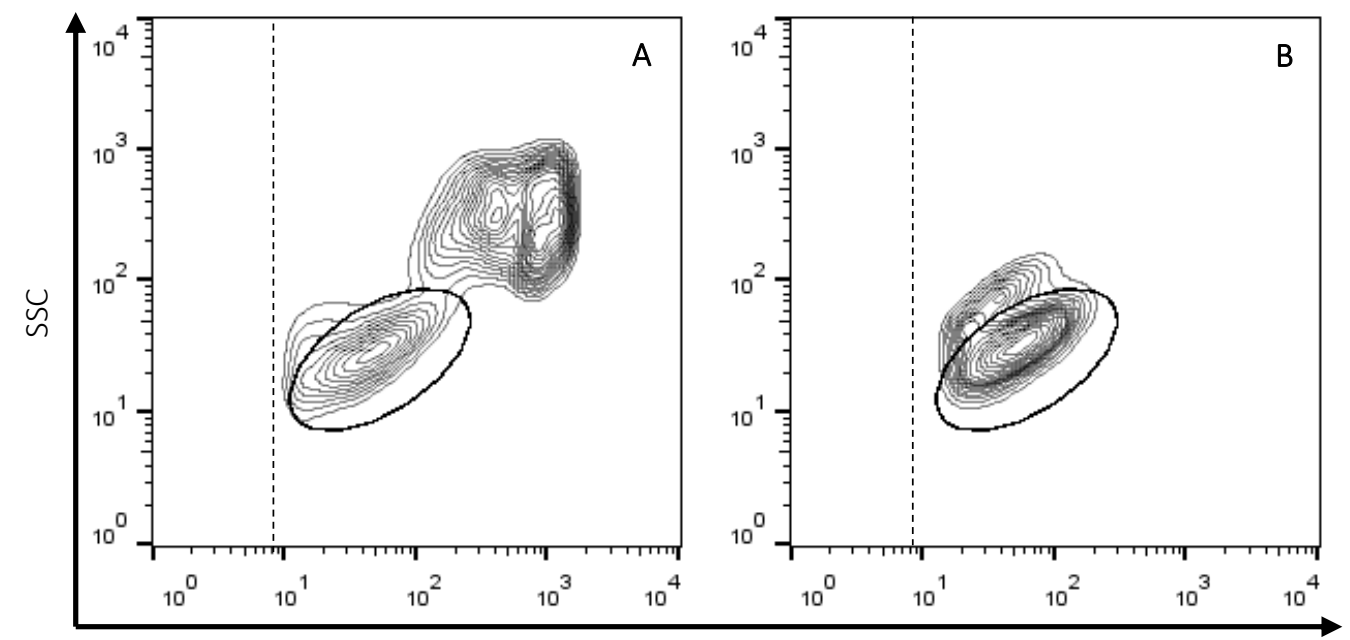

FSC
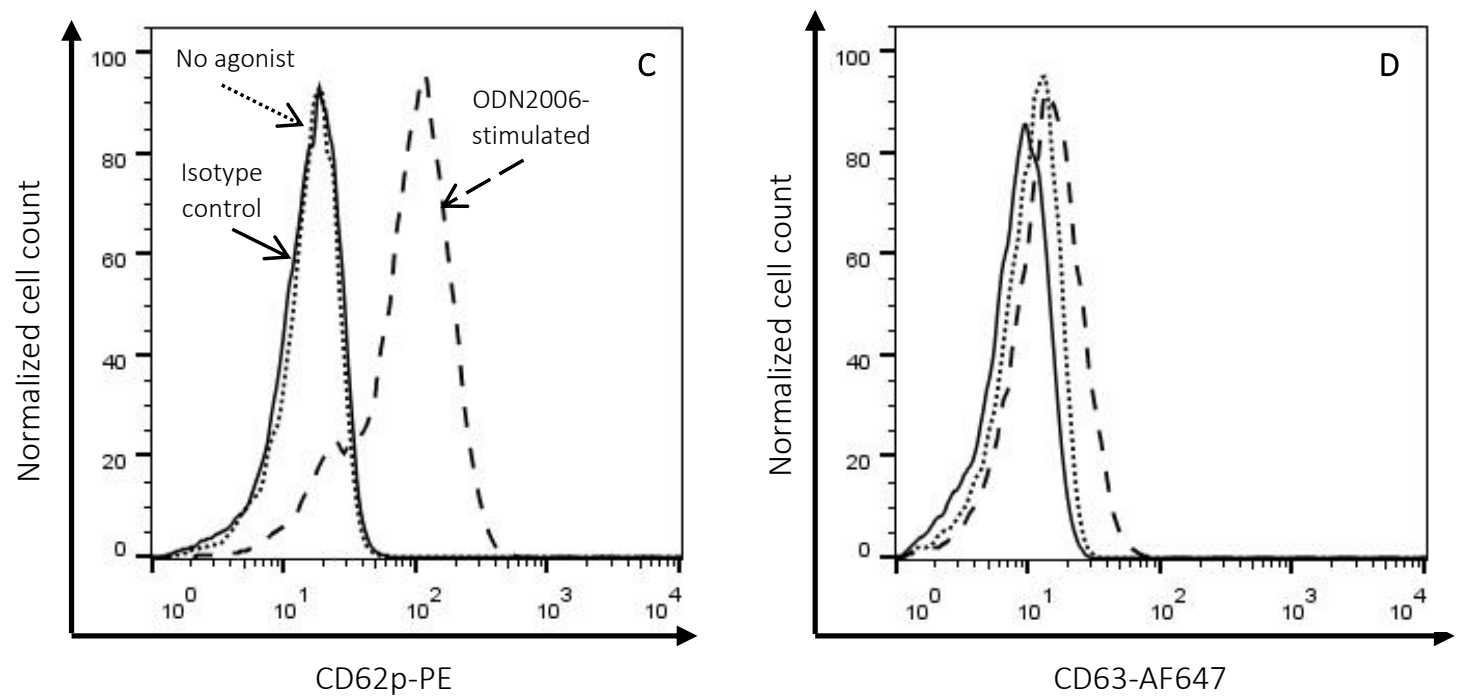

Figure 2.3 Identification of the platelet population and of the platelet activation markers, CD62p and CD63.

Platelets were identified by their side scatter and forward scatter properties for both WB (A) and PRP (B) and, further, by their CD42a-positivity (as described in Figure 2.1). Representative plots of CD62p (C) and CD63 (D) expression are shown for unstimulated and ODN2006-stimulated platelets. 


\subsubsection{Statistical analysis.}

Because the difference in platelet-TLR9 expression or the difference in TLR9-mediated platelet activation between a healthy and diseased cohort has not yet been studied, we did not have data on the variance of these measurements to properly estimate an appropriate sample size. Therefore, for each cohort, we empirically chose to recruit 5 subjects to examine TLR9 expression and 16 subjects to examine TLR9-mediated platelet activation, respectively.

Differences in expression of platelet activation markers or TLR9 expression within each cohort were examined using one-way ANOVA. Where significant differences were found to exist, posthoc paired t-tests were performed. All values are reported as mean \pm standard deviation (SD). Differences between cohorts were examined using unpaired t-tests. For demographics data, continuous variables were analysed using an unpaired t-test and reported as mean \pm SD and categorical variables were analysed using a Chi-squared test and reported as frequency (percentage). Statistical analysis was performed using GraphPad Prism 6 (GraphPad Software Inc., La Jolla, CA, USA). 


\subsection{Results.}

\subsubsection{TLR9 expression in healthy and AMI subjects.}

During the study period, 5 healthy and 5 AMI subjects were enrolled to examine TLR9 expression. The healthy cohort had a mean age of 26 years and 2 were male. The mean age of AMI patients was 66, 4 were male. 3 AMI subjects presented with an NSTEMI and 2 presented with a STEMI.

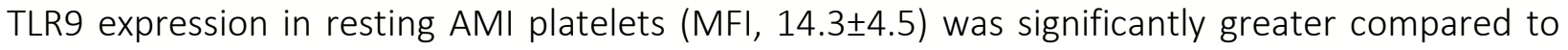
healthy subjects (MFI 7.2 $\pm 2.5, \mathrm{p}<0.01$; Figure 2.4A). In the healthy cohort, a dose-dependent increase in TLR9 expression was reported for $1 \mu \mathrm{M}$ TRAP-activated platelets (MFI 9.8 \pm 2.7 , ns) and $10 \mu \mathrm{M}$ TRAP-activated platelets (MFI 12.8 \pm 3.6 , $\mathrm{p}<0.05$ ) in comparison to unstimulated platelets.

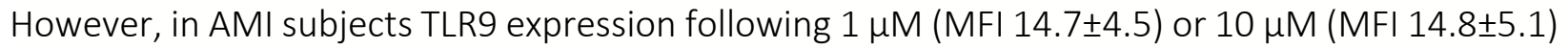
TRAP activation was similar to TLR9 expression seen in unstimulated platelets. Cell-surface CD62p expression, a platelet activation marker, was examined to ensure sufficient TRAP-mediated platelet activation was achieved across both cohorts (Figure 2.4B). The percentage of the platelet population expressing cell-surface CD62p expression was low in unstimulated platelets and no different across the cohorts (healthy cohort, $2.3 \%$ of the platelet population was CD62p-positive; ASC cohort, 1.8\%). CD62p expression was mildly elevated in both healthy (23.6\%) and AMI (15.5\%) subjects in response to $1 \mu \mathrm{M}$ TRAP and markedly elevated in response to $10 \mu \mathrm{M}$ TRAP (healthy, 74.3\%; AMI, 66.8\%). The trends in TLR9 expression described above were qualitatively verified by western blot (Figure 2.4C); elevated TLR9 protein levels were seen in AMI platelets, across all treatments, in comparison to resting healthy platelets. 

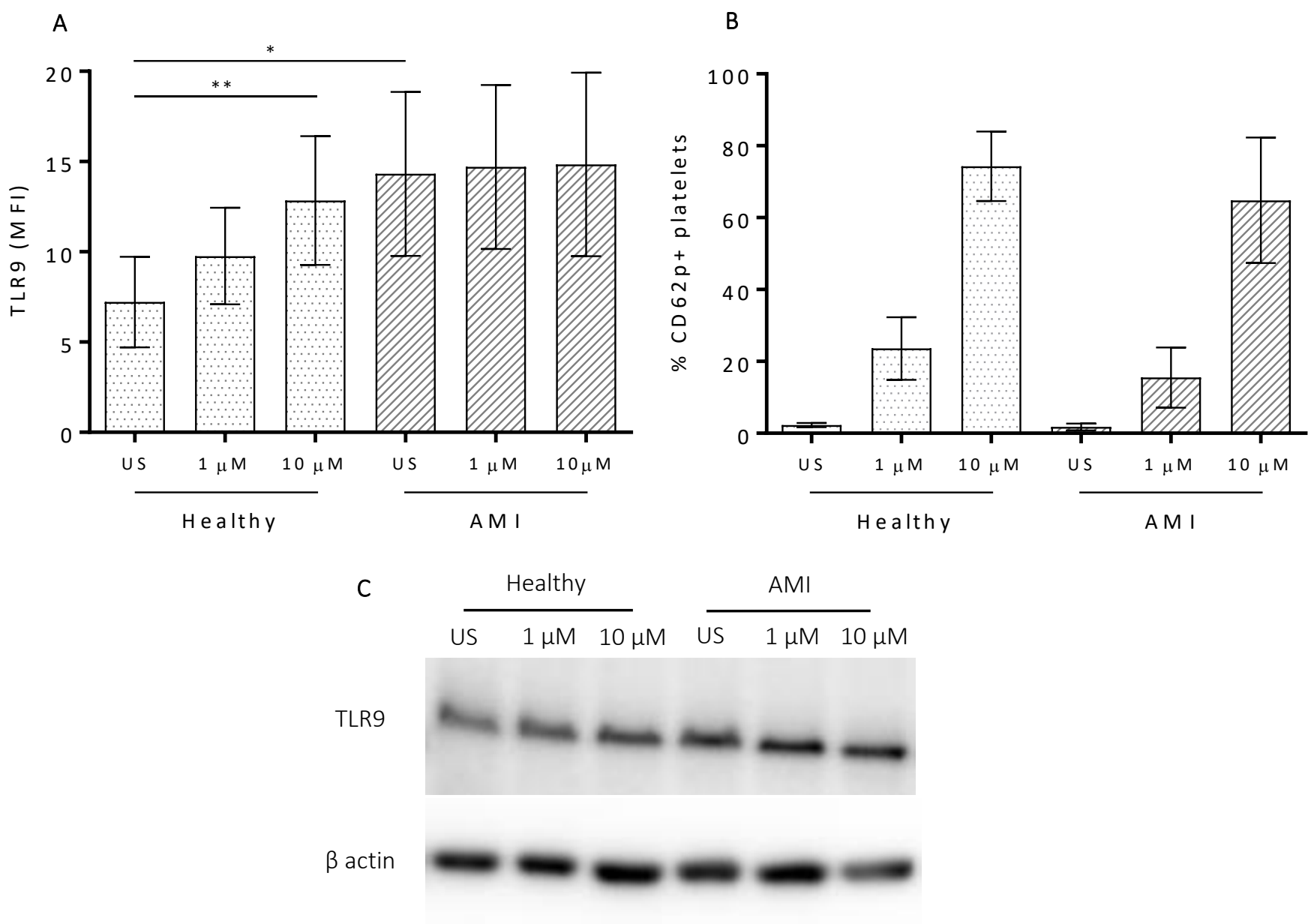

Figure 2.4 TLR9 expression increases in AMI platelets and upon platelet activation, compared to resting healthy platelets.

Platelet-TLR9 expression, as measured by MFI, was analysed in response to $1 \mu \mathrm{M}$ and $10 \mu \mathrm{M}$ TRAP stimulation (A). Mean \pm SD for 5 subjects in each cohort are shown. For TLR9 expression, one way ANOVA and paired t-tests were performed to detect differences within each cohort and unpaired t-tests were performed to detect differences between cohorts. * $p<0.05, * * p<0.01$. Cell-surface CD62p expression was assessed to ensure sufficient platelet activation under the TRAP stimulation conditions (B). Western blotting confirms the trends seen by flow cytometry (C). 


\subsubsection{Dose response curves for ODN2006-mediated platelet activation.}

In order to establish a dose-response curve for platelet activation in response to ODN2006 stimulation, PRP from 5 healthy individuals was incubated with $0.156 \mu \mathrm{M}$ to $10 \mu \mathrm{M}$ ODN2006, a TLR9 agonist, or TRAP (Figure 2.5). Maximal platelet activation, as measured by CD62p expression, occurred following incubation with 5 MM ODN2006. This concentration was, therefore, used for the subsequent platelet activation studies. $2.5 \mu \mathrm{M}$ and $10 \mu \mathrm{M}$ TRAP produced sub-maximal and maximal platelet activation, respectively, and were used as positive controls for subsequent platelet activation studies.

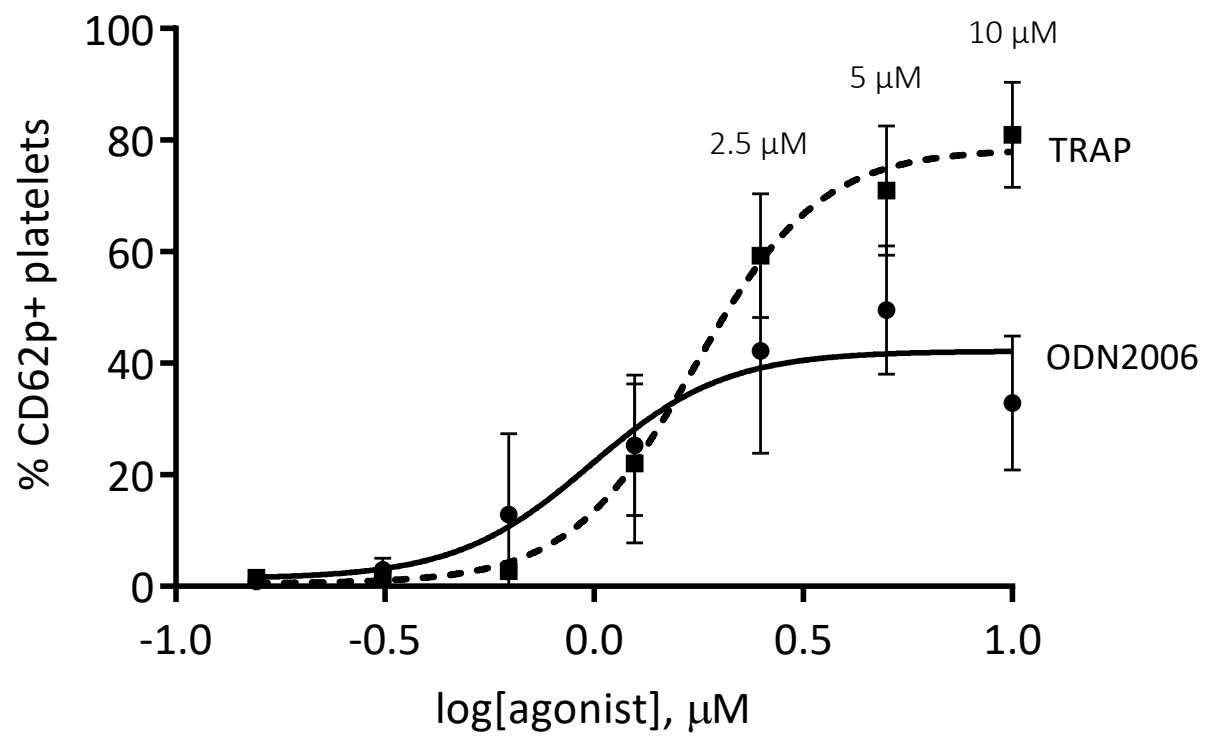

Figure 2.5 Dose-response curves for TRAP and ODN2006.

The percentage of the platelet population that was CD62p-positive, which indicates an activated platelet, was measured following incubation with $0.156 \mu \mathrm{M}-10 \mu \mathrm{M}$ TRAP, a universal platelet agonist, and ODN2006, a TLR9 agonist. Agonist concentrations were log transformed and a sigmoidal curve was fitted. Mean \pm SD for 5 healthy subjects is shown. 


\subsubsection{Demographics for ODN-mediated platelet activation.}

16 healthy and 16 AMI subjects were enrolled to examine ODN2006-mediated platelet activation, separate from the subjects that were enrolled to examine TLR9 expression. The baseline demographics and clinical characteristics of both cohorts enrolled for this part of the study are given in Table 2.3. The healthy cohort had a mean age of 52 years and 9 (56\%) were male. The AMI cohort were older than the healthy group ( $64 \pm 11$ years vs. $52 \pm 8$ years, $p<0.001$ ) but had a similar gender distribution (56\%) and body mass index ( $29 \pm 6$ vs. $26 \pm 4, p=0.12$ ). In the AMI cohort, $94 \%$ presented with a NSTEMI, 19\% were diabetic, and the prevalence of hypertension (63\%) and dyslipidaemia (69\%) was high. All AMI subjects were pre-treated with DAPT; 9 subjects received clopidogrel with aspirin and 7 received ticagrelor with aspirin. 
Table 2.3 Demographics and clinical characteristics of the AMI and healthy cohort.

\begin{tabular}{|c|c|c|c|}
\hline & $\begin{array}{l}\text { AMl cohort } \\
(n=16)\end{array}$ & $\begin{array}{l}\text { Healthy cohort } \\
(n=16)\end{array}$ & $\mathrm{p}$-value \\
\hline \multicolumn{4}{|l|}{ Demographics } \\
\hline Age, years & $64 \pm 11$ & $52 \pm 8$ & $p<0.001$ \\
\hline Male & $9(56)$ & $9(56)$ & -- \\
\hline $\mathrm{BMI}, \mathrm{kg} / \mathrm{m}^{2}$ & $29 \pm 6$ & $26 \pm 4$ & $p=0.12$ \\
\hline \multicolumn{4}{|l|}{ Cardiovascular risk factors } \\
\hline Hypertension & $10(63)$ & $0(0)$ & -- \\
\hline Dyslipidaemia & $11(69)$ & $0(0)$ & -- \\
\hline Diabetes & $3(19)$ & $0(0)$ & -- \\
\hline Current smoker & $1(6)$ & $0(0)$ & -- \\
\hline Renal dysfunction & $1(6)$ & $0(0)$ & -- \\
\hline \multicolumn{4}{|l|}{ Clinical presentation } \\
\hline STEMI & $1(6)$ & $0(0)$ & -- \\
\hline NSTEMI & $15(94)$ & $0(0)$ & -- \\
\hline \multicolumn{4}{|l|}{ Anti-platelet medication } \\
\hline Aspirin & $16(100)$ & $0(0)$ & -- \\
\hline Clopidogrel & $9(56)$ & $0(0)$ & -- \\
\hline Ticagrelor & $7(44)$ & $0(0)$ & -- \\
\hline
\end{tabular}

All continuous variables were reported as mean \pm standard deviation and all categorical variables were reported as frequency (percentage). BMI, body mass index; STEMI, ST-elevation myocardial infarction; NSTEMI, non-STelevation myocardial infarction. 


\subsubsection{Platelet activation in healthy subjects following ODN2006 agonism.}

At a group level, ODN2006 resulted in a significant increase in the percentage of CD62p-positive platelets $(55 \%$ increase from no agonist, $\mathrm{p}<0.0001$ ) in healthy WB (Figure 2.6A). Similarly, a significant increase in CD63 expression was observed across the whole platelet population (MFI, 0.61 in no agonist vs. 3.81 in ODN2006 stimulated sample, $p<0.0001$, Figure 2.6C). Similar to the response in WB, ODN2006 stimulation significantly increased CD62p (46\% increase, $p<0.0001$, Figure 2.6B) and CD63 expression (MFI 1.63 vs. 3.17, $p<0.001$, Figure 2.6D) in healthy PRP in comparison to unstimulated platelets. Stimulation of platelets with both doses of TRAP produced significant increases in both activation markers across WB and PRP. 

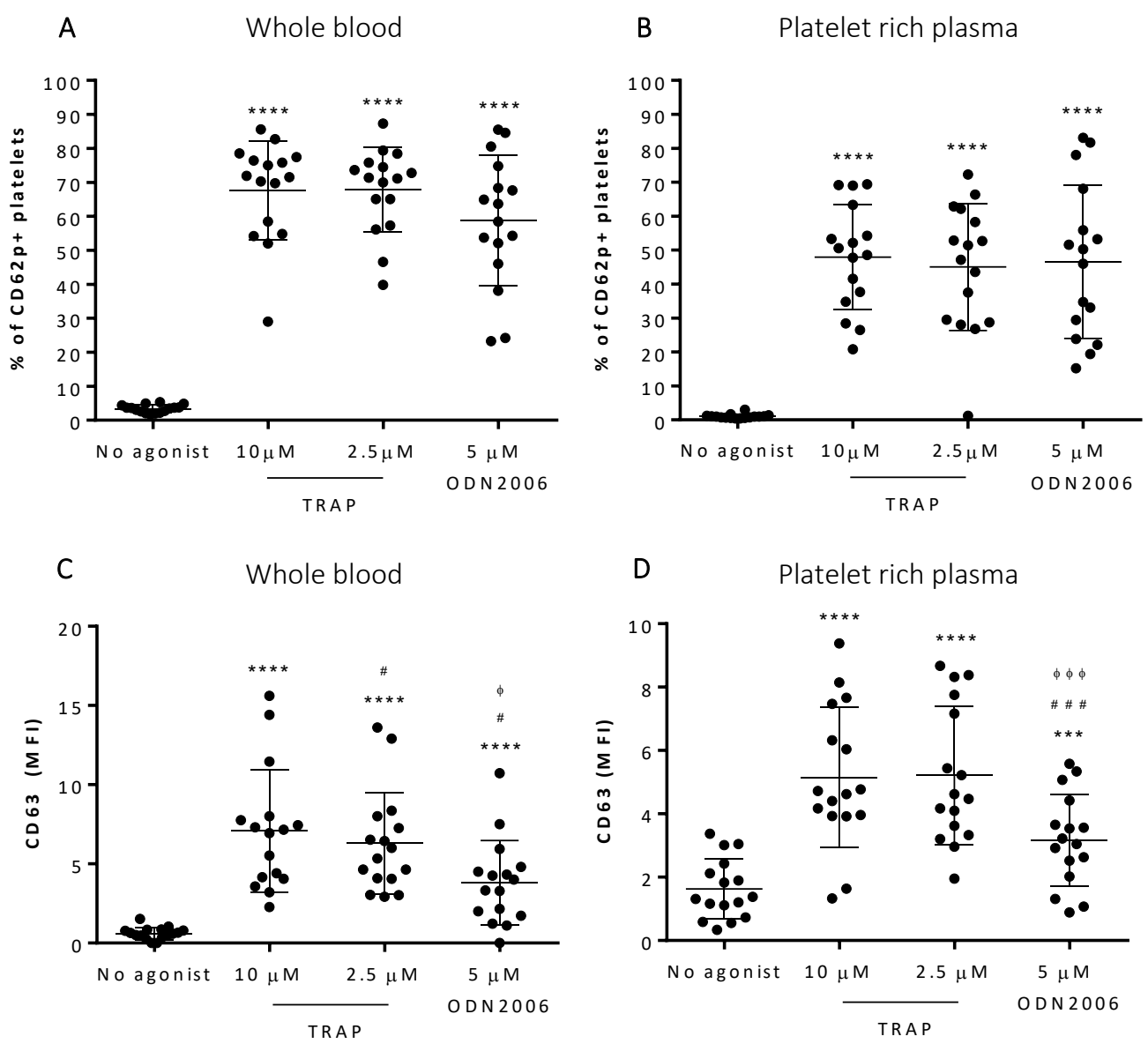

Figure 2.6 Incubation with ODN2006 in WB and PRP induced significant platelet activation in healthy subjects.

CD62p (A, B) and CD63 (C, D) expression in response to TRAP and ODN2006 was analysed in WB (A, C) and PRP (B, D). Mean \pm SD for 16 subjects in each cohort are shown. Within each cohort, one way ANOVA was found to be significant $(p<0.05)$ for each parameter and allowed for paired t-tests to be performed. * denotes significance in relation to the no agonist sample, ${ }^{\phi}$ in relation to $2.5 \mu \mathrm{M}$ TRAP, ${ }^{*}$ in relation to $10 \mu \mathrm{M}$ TRAP. $\#, \phi p<0.05, * * *, \# \#, \phi \phi \phi p<0.001, * * * *$ $p<0.0001$. 


\subsubsection{Platelet activation in AMI subjects following ODN2006 agonism.}

In response to ODN2006, there was a significant increase in both CD62p (61\% increase from no agonist, $p<0.0001$, Figure 2.7A) and CD63 expression (MFI 0.75 in no agonist vs. 4.84 in ODN2006 stimulated, $p<0.0001$, Figure 2.7C) in AMI WB when compared to unstimulated platelets. Similarly, ODN2006-mediated platelet activation in AMI PRP was significantly larger than that observed in the no agonist sample for both CD62p (47\% increase, $p<0.0001$, Figure 2.7B) and CD63 (MFI 0.67 vs. 2.41, $p<0.0001$, Figure 2.7D). As noted in the healthy cohort, there was a significant increase in TRAP-mediated platelet activation in WB and PRP. 

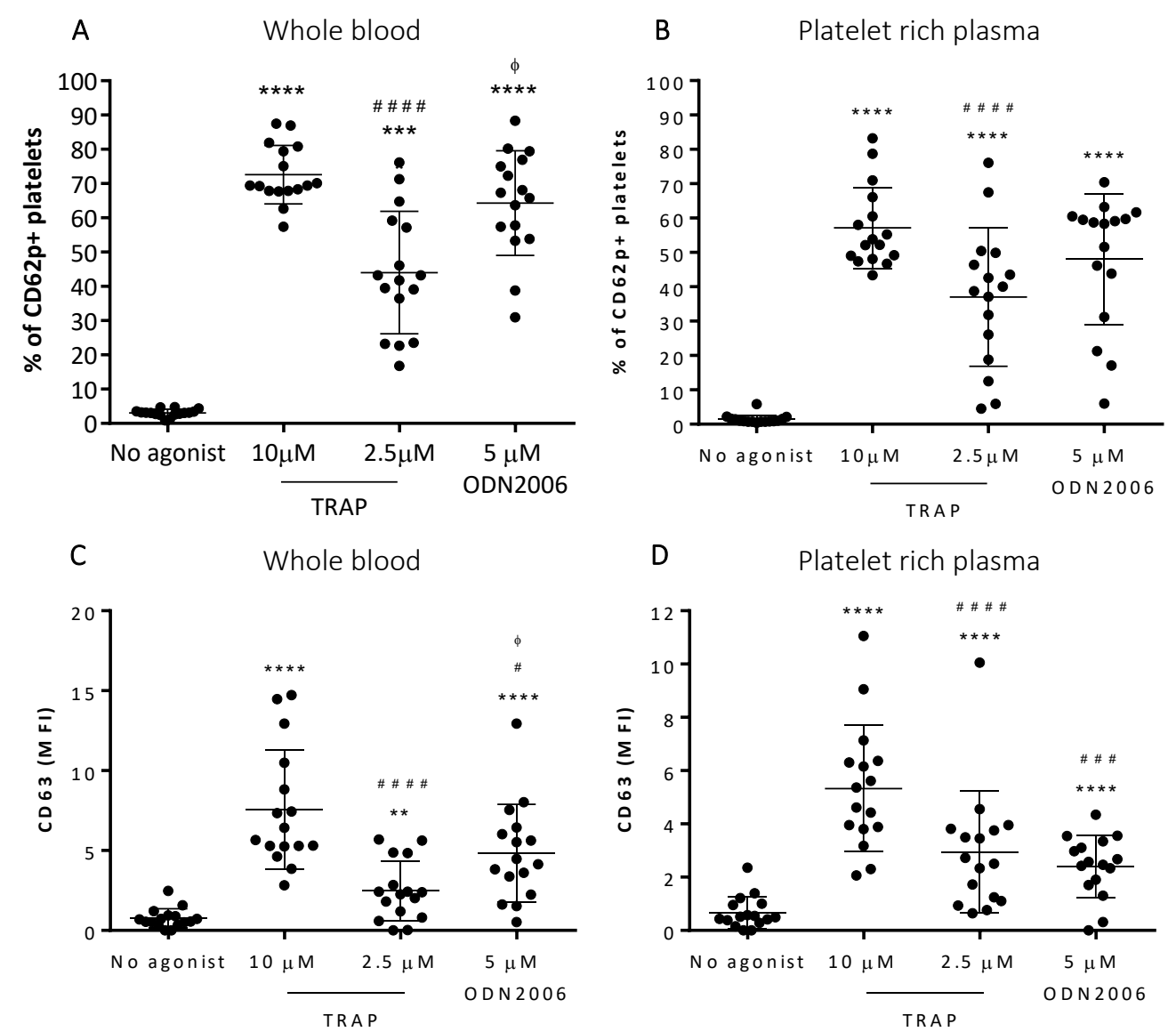

Figure 2.7 Incubation with ODN2006 in WB and PRP induced significant platelet activation in AMI subjects.

CD62p (A, B) and CD63 (C, D) expression are shown in response to TRAP and ODN2006 was analysed in WB (A, C) and $\operatorname{PRP}(B, D)$. Mean $\pm S D$ for 16 subjects in each cohort is shown. Within each cohort, one way ANOVA was found to be significant $(p<0.05)$ for each parameter and allowed for paired t-tests to be performed. ${ }^{*}$ denotes significance in relation to the no agonist sample, ${ }^{\phi}$ in relation to $2.5 \mu \mathrm{M}$ TRAP, ${ }^{*}$ in relation to $10 \mu \mathrm{M}$ TRAP. ${ }^{*}, \phi p<0.05,{ }^{* *} p<0.01$, $\# \#$ \# $<0.001, * * * *$,\#\#\#\# $\mathrm{p}<0.0001$. 


\subsubsection{Comparison of PRP and WB responses to ODN2006.}

There was a significantly greater increase in the percent of platelets expressing CD62p following ODN2006 stimulation in WB than in PRP in both the healthy (12\% increase, $p<0.01)$ and AMI (16\% increase, $\mathrm{p}<0.01$ ) cohort (Figure 2.8A). Similarly, there was a greater increase in CD63 expression in WB when compared to PRP for the AMI cohort (MFI 4.84 in WB vs. 2.41 in PRP, p<0.01, Figure 2.8B). However, the increase in CD63 expression in the healthy cohort following ODN stimulation was not statistically different in WB compared with PRP (MFI 3.81 vs. 3.17, $p=0.23$ ).
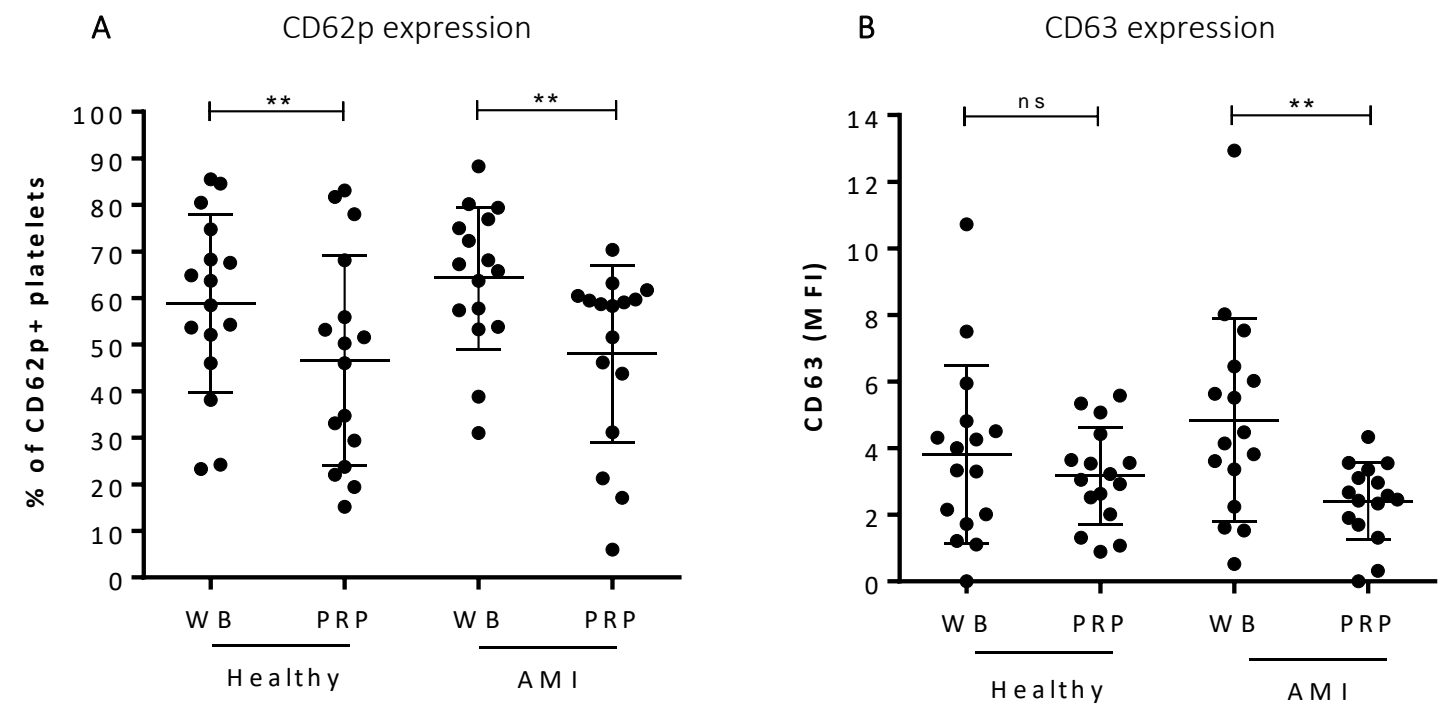

Figure 2.8 Platelet activation in response to ODN2006 is greater in WB than in PRP.

Platelet activation between WB and PRP was analysed by CD62p (A) and CD63 (B) expression in both cohorts. Within each cohort, paired t-tests were performed to detect differences. ${ }^{* *} p<0.01$. 


\subsubsection{Comparison of ODN2006-mediated platelet activation across cohorts.}

A similar response to ODN2006 was seen across both the AMI and healthy cohorts, shown in Figure 2.9. CD62p expression in healthy WB (59\% of platelets were CD62p-positive) and PRP (47\%) was not different to that of AMI WB (64\%) and PRP (48\%). There was also no difference in CD63 expression recorded for WB (MFI 3.81 in healthy vs 4.84 in AMI cohort) and PRP (MFI 3.17 vs 2.41).
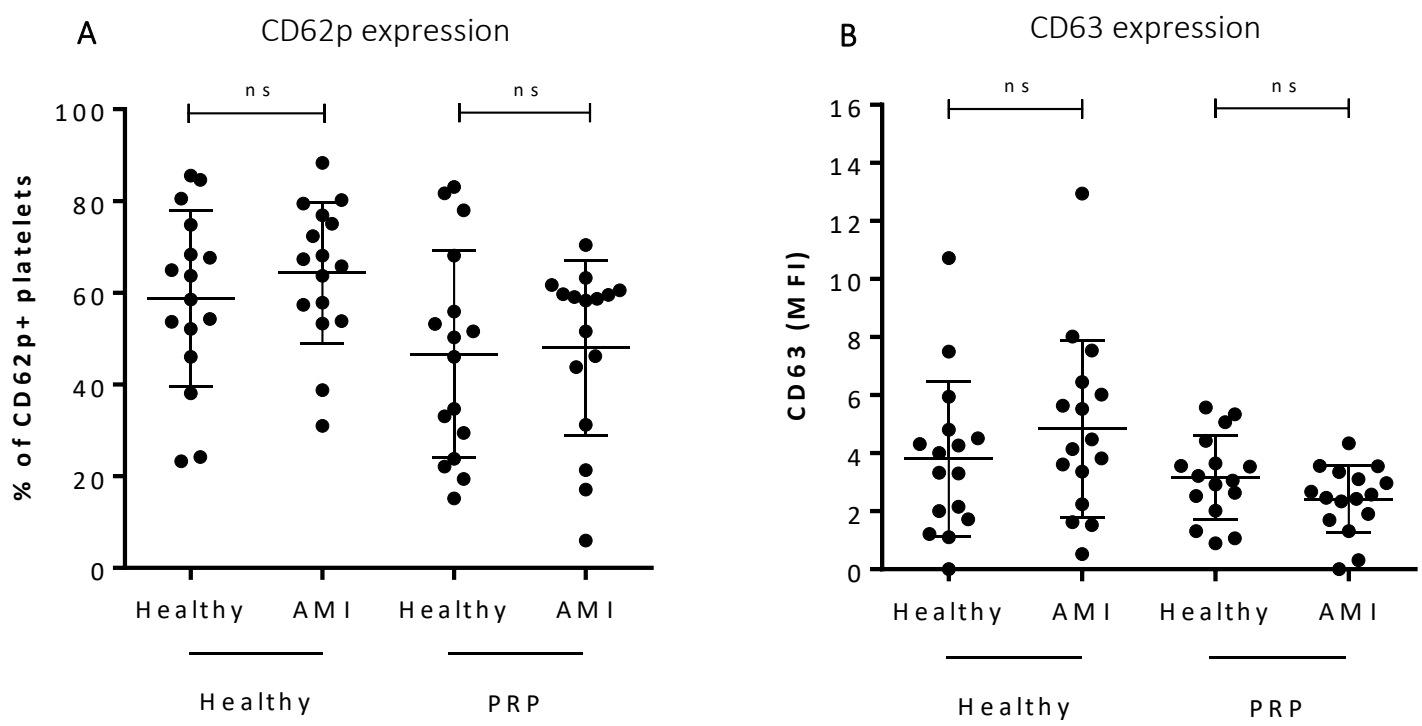

Figure 2.9 Platelet activation in response to ODN2006 is similar across cohorts.

Platelet activation between the healthy and AMI cohort was analysed by CD62p (A) and CD63 (B) expression in both WB and PRP. Unpaired t-tests were performed to detect differences between cohorts. 


\subsubsection{A differential platelet response to 2.5 $\mu M$ TRAP was seen between cohorts.}

Compared to healthy WB, platelet CD63- and CD62p-positivity were attenuated in AMI WB in response to $2.5 \mu \mathrm{M}$ TRAP (CD62p, 24\% absolute decrease; CD63, 3.8\% absolute decrease; both $p<0.001 ;$ Figure 2.10). The same trend can be described for the results in PRP; CD62 $p$ expression ( $8 \%$ absolute decrease, $p=0.25$ ) and CD63 expression ( $2.3 \%$ absolute decrease, $p<0.01$ ) is higher in the healthy cohort.

A

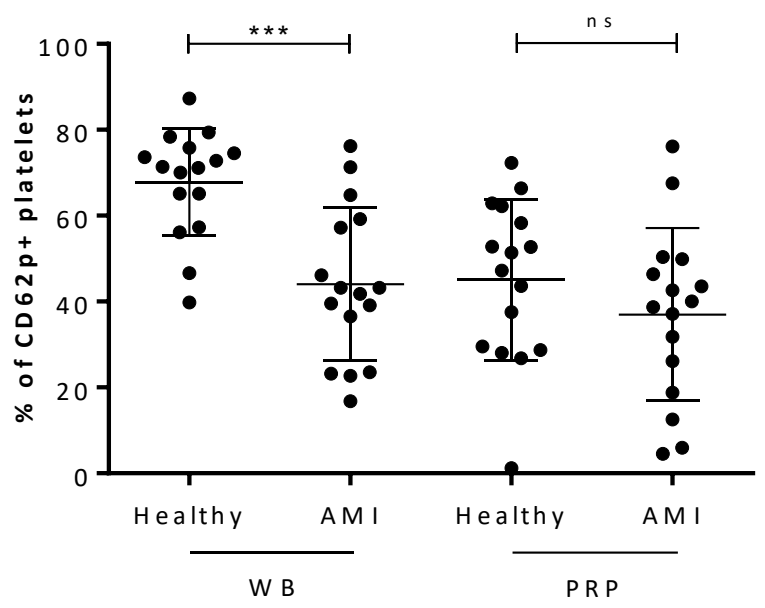

B

CD63 expression

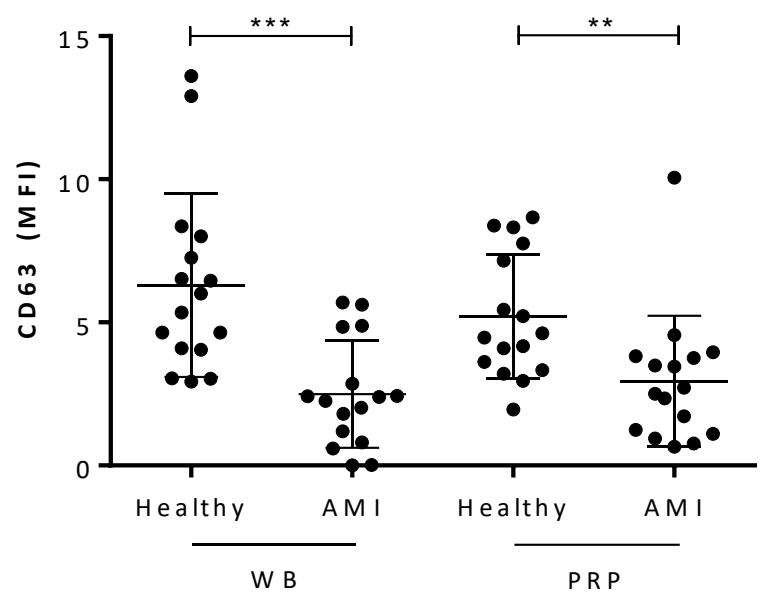

Figure 2.10 Platelet activation in response to $2.5 \mu \mathrm{M}$ TRAP in WB is greater in the healthy cohort.

The AMI platelet population becomes less activated in response to $2.5 \mu \mathrm{M}$ TRAP, in comparison to healthy platelets for both CD62p (A) and CD63 (B) expression. Unpaired Student's t-tests were performed, $* * p<0.01, * * * p<0.001$. 


\subsection{Discussion.}

In this study, we demonstrated that platelet-TLR9 expression is significantly higher in AMI subjects, compared to healthy subjects. Furthermore, TLR9 dose-dependently increased in response to TRAP activation in the healthy cohort, although this was not observed in the AMI cohort. Stimulation with ODN2006, a TLR9 agonist, produced significant platelet activation in PRP and WB across both cohorts. Interestingly, the level of ODN2006-mediated platelet activation was similar across cohorts, despite treatment with DAPT in the AMI cohort.

Consistently, TLRs have been implicated in the development and progression of atherosclerosis. Immune cell-TLR activation has been reported to be pro-atherogenic, resulting in increased plaque infiltration and cytokine release and, ultimately, exacerbating the pro-inflammatory environment at the atherosclerotic plaque site $[171,174]$. Platelets are increasingly implicated in immune responses at the plaque site and are known to be pathogenic in AMI. Platelet-TLRs may act as a link between thrombosis and inflammation, but platelet-TLR expression and activation is not well characterised in atherosclerosis or AMI.

Elucidating TLR function in healthy platelets remains the focus throughout the current literature. However, the significance of TLR expression and function in disease states where platelets are known to play a large and detrimental effect has yet to be fully addressed. Inappropriate platelet activation in AMI patients increases the risk of experiencing recurrent MACE [248], representing a real and significant concern for clinicians. Stimulating alternative platelet activation pathways may contribute to this risk, but these remain relatively unexplored. We are the first to demonstrate that platelet-TLR9 expression is increased in AMI and that ODN2006 stimulation causes platelet activation in AMI patients, despite treatment with DAPT. We suggest that the platelet-TLR9 pathway is an alternative platelet activation pathway that may be of therapeutic importance in AMI. 
TLR9 expression has been variably reported in healthy platelets previously $[187,188]$, residing in tubular system-related compartments situated towards the periphery of the platelet [215]. We demonstrate elevated TLR9 expression in AMI platelets when compared to healthy platelets. The difference in TLR9 expression between a healthy state and a diseased state, such as AMI, indicates dynamicity of platelet function and reactivity during disease. It is possible that AMI platelets have an entirely different phenotype, which reflects the pro-inflammatory and pro-thrombotic environment that arises during an AMI, compared to healthy individuals. Increased platelet-TLR9 expression may represent a pro-inflammatory state which predisposes AMI platelets to elevated sensitivity towards TLR9 agonists. Ultimately, such sensitivity could contribute to high platelet reactivity in $\mathrm{AMI}$ and increased involvement in both the thrombotic and immune responses directed against the ruptured atherosclerotic plaque environment.

We have demonstrated that TLR9 expression increases upon activation in healthy platelets. Further to this, maximally stimulating healthy platelets with $10 \mu \mathrm{M}$ TRAP resulted in TLR9 expression levels similar to that seen in unstimulated AMI platelets. These findings suggest that platelet activation is a likely mechanism to explain increased TLR9 expression in AMI platelets. AMI patients experience extensive platelet activation during their event [249] and it is possible that platelet-TLR9 upregulation occurs as a by-product of this activation. Stimulating AMI platelets further does not result in any additional increase in expression. We suggest TLR9 expression is upregulated to a maximum level at some point during the AMI event.

AMI patients were recruited into this study an average of 4 days following symptom onset, which suggests platelet-TLR9 is upregulated during AMI and remains elevated post-AMI. Following AMI, the atherosclerotic plaque environment remains highly pro-thrombotic and pro-inflammatory and, during this time, patients are at risk of experiencing recurrent ischaemic events. Increased platelet sensitivity to TLR9 antigens post-AMI may contribute to this increased risk. 
With this in mind, we aimed to determine the extent to which ODN2006, a TLR9 agonist [250, 251], activated platelets from AMI platelets treated with DAPT. ODN2006 agonsim resulted in significant platelet activation in both healthy subjects and in AMI subjects treated with DAPT. ODN2006 resulted in a significant increase in expression of both CD62p and CD63 in PRP, suggesting direct activation of platelet-TLR9. Previous studies have reported TLR9-mediated platelet activation in similar platelet-only suspensions $[215,216]$, demonstrating TLR9 to be a functional platelet receptor. In WB, the expression of both activation markers in response to ODN2006 was greater than the response in PRP, which suggests an indirect component of platelet activation. A wide range of leukocytes express TLR9 to varying degrees [252-254] and TLR9 agonism induces various cell-specific responses [255-258]. These responses include leukocyte activation and release of immunomodulators, both of which can result in platelet activation [259, 260]. The various indirect effects of ODN2006 in WB provide a mechanism for the greater level of activation seen in this medium, when compared to PRP.

TLRs recognize a number of PAMPs and DAMPs, and engagement of these receptors rapidly triggers a series of innate immune responses. The platelet-TLR9 response to DNA-derived PAMPs may provide a link between infection, platelet activation and increased risk of AMI. Many groups have demonstrated the seasonal variation in admission to hospital [261, 262] and death [263] due to AMI. All report a peak incidence in winter, concurrent with a peak in infection rates. Alongside these reports, there is mounting evidence to link acute infection with the development of AMI $[264,265]$. The persistent and high inflammatory state which exists in response to infection increases plaque infiltration of inflammatory cells and local secretion of pro-inflammatory cytokines [266]. This environment may transiently increase platelet-TLR activation, thus increasing the risk of thrombosis and susceptibility to an $\mathrm{AMI}$ as a result.

Although TLR activation has traditionally been described in response to external infection, responses to internal 'dangerous' self-ligands have also been recently characterized [267-269]. Stimulating platelet-TLR9 by such DAMPs is also a mechanism by which TLRs cause platelet 
activation and increased risk of thrombosis. Platelet-TLR9 has recently been shown to respond to CAPs, formed as a by-product of phospholipid oxidation during oxidative stress [216]. Responses included granule secretion and aggregation in vitro and thrombosis in vivo, demonstrating plateletTLR9 to be a novel connection between oxidative stress, innate immunity and thrombosis.

All AMI patients were sufficiently loaded with DAPT (aspirin with either clopidogrel or ticagrelor) at the time of blood collection. Anti-platelet therapy did not significantly alter the platelet response to high-dose (10 $\mu \mathrm{M})$ TRAP, when compared to healthy subjects, but did inhibit the response to low-dose $(2.5 \mu \mathrm{M})$ TRAP. These results indicate that DAPT is sufficient to inhibit TRAPmediated platelet activation at a dose that produces sub-maximal platelet activation. However, a similar response to ODN2006 stimulation was seen across both cohorts, indicating a lack of inhibition of ODN2006-mediated platelet activation (at the dose tested in this study) by DAPT. Based on these results, we suggest that platelet activation via the TLR9 pathway may not be adequately inhibited by guideline-recommended anti-platelet therapy and may contribute to persistent on-treatment platelet activation post-AMI.

CPG ODNs, which are recognized by TLR9, have potent immunostimulatory effects and have been used as adjuvants to a number of monoclonal antibody and vaccine therapies previously (for a review, see [270]). In mouse models, CpG ODNs can enhance the efficacy of the immune response to hepatitis B (HB) surface antigen [271] and tetanus toxoid [272], to name a few. As an example of the use of CpG ODNs in a phase I clinical trial, the efficacy of the commercial HB vaccine (EnergixB) was improved with the use of CpG ODN7909 as an adjuvant, with the HB-specific antibody response appearing significantly sooner in subjects receiving this adjuvant, compared to control subjects [273]. In our study, we demonstrate potent in vitro platelet activation in response to ODN2006. These results suggest that a possible side-effect of the clinical use of CpG ODNs may be an increase in in vivo platelet activation. We suggest that platelet activation is a parameter that needs to be monitored, particularly in participants with a higher risk of thrombosis, in clinical situations that use ODNs. 
We have described a platelet activation pathway for which the cellular mechanism is, as yet, unknown. TLR9 activation in anucleate platelets must be fundamentally different to that in nucleated immune cells, where the 'end-point' of TLR signalling is the nuclear translocation of transcription factors. Our results suggest that the TLR9 signalling pathway feeds into traditional platelet activation pathways. Several groups have identified key TLR signalling components in platelets including MyD88, TRIF and NF-KB $[213,274]$ suggesting a relatively intact, although perhaps non-classical, TLR9 signalling pathway. The point at which these two distinct pathways interact serves as a potential therapeutic target to reduce platelet activation. Elucidating the signalling pathway that is initiated by TLR9 engagement and ends in platelet activation may provide viable targets for this purpose. 


\subsection{Limitations.}

TLR9 expression and ODN2006-mediated platelet activation were investigated in healthy subjects that were younger than the AMI cohort, and we cannot exclude a possible effect of age on these results. We have examined the relationship between age and platelet-TLR9 expression ( $n=5)$ as well as age and platelet activation in response to ODN2006 $(n=16)$ in the AMI group and in the healthy group separately. We found no evidence that age is a significant determinant of expression or activation, but as we have not controlled for this, we cannot be certain that it is not a contributor. For ODN2006-mediated platelet activation, a larger AMI cohort would have allowed for greater statistical power for analysis of platelet activation under different DAPT regimens (aspirin with clopidogrel vs. ticagrelor). However, this study achieved the aim of describing the platelet response to ODN2006 at the cohort level, and was not designed to answer sub-group analysis questions. It is not clear what concentration of TLR9 agonists can be achieved in the blood of infectious patients or patients with a sterile inflammatory condition, and therefore what would constitute a clinically or physiological relevant concentration for use in this study. However, the dose of ODN2006 used in this study was consistent with previous literature [215]. We cannot exclude off-target effects of ODN2006, but it is a widely-used TLR9 agonist $[250,251]$ and has been used to assess platelet activation previously $[215,216]$. We attempted to assess the specificity of ODN2006 for platelet-TLR9 by blocking TLR9 signalling with an inhibitory peptide against the downstream signalling molecule, MyD88. However, across varying concentrations and incubation times, the MyD88 inhibitory peptide induced significant platelet activation. We investigated other methods for inhibiting this pathway, such as the use of commercially available neutralizing antibodies, but these antibodies are only available for cell-surface expressed TLRs. As a result, we were unable to determine the extent of platelet activation that could be achieved by ODN2006 in the absence or presence of TLR9 inhibition. 


\subsection{Conclusion.}

We have demonstrated an alternative platelet activation pathway that causes potent platelet activation in both healthy and AMI subjects. Platelet-TLR9 expression was significantly elevated in those subjects with AMI compared to healthy subjects which could result in increased sensitivity to TLR9 agonists. Platelet activation caused increased TLR9 expression in healthy platelets. We suggest that platelet activation, which occurs as a part of an AMI, is a potential mechanism to explain the increased platelet-TLR9 expression seen in AMI patients. ODN2006-mediated platelet activation in PRP demonstrates a direct effect of ODN2006 acting on the platelet population. The larger response seen in WB suggests additional indirect activation by stimulation of other 'offplatelet' TLR9 pathways. We are the first to demonstrate ODN2006-mediated platelet activation in AMI patients on DAPT, indicating that the TLR9 pathway is inadequately inhibited by current anti-platelet drugs. As such, the platelet-TLR9 pathway may contribute to platelet activation postAMI and despite treatment with DAPT. If the platelet-TLR9 pathway is activated in the setting of $\mathrm{AMI}$, this may increase the risk of thrombosis and the risk of adverse cardiovascular events in these patients. The platelet-TLR9 pathway may serve as a potential target for therapeutic intervention in AMI. 
3 Chapter 3. Platelet Toll-like receptor 1, 2, 4 and 6 expression and TLR-mediated platelet activation in AMI.

Publication arising from this chapter:

Hally, K. E., La Flamme, A. C., Larsen, P. D., \& Harding, S. A. (2017). Platelet Toll-like receptor (TLR) expression and TLR-mediated platelet activation in acute myocardial infarction. Thrombosis Research, 158, 8-15. doi: https://doi.org/10.1016/j.thromres.2017.07.031 


\subsection{Introduction.}

In this chapter, we wanted to extend our assessment of platelet-TLR expression and TLR-mediated platelet activation to include TLR1, 2, 4 and 6, which are predominantly expressed on the platelet surface. From the previous chapter, we suggest that increased TLR9 expression and TLR9mediated activation of platelets in AMI patients may provide a pathway for ongoing platelet activation post-AMI and despite treatment with DAPT. It is important, therefore, to widen our assessment to consider all platelet-TLR pathways that may contribute to ongoing platelet activation in this group of patients.

Alongside TLR9, expression and activation of TLR1, 2, 4 and 6 has been most well-characterized in platelets from healthy subjects $[184,187-189,198]$. These studies have shown that platelets express TLR1, 2 and 6 at low levels and express a high level of TLR4. However, it is not known how total levels of platelet-TLR1, 2, 4 and 6 expression change during AMI. Healthy platelets have been shown to become activated in response to TLR4 and TLR2/1 stimulation, but not TLR2/6 stimulation, previously. We do not yet know whether platelet activation in response to stimulation of these TLRs changes in AMI, or what functional significance these platelet-TLR pathways have in individuals treated with DAPT. On the basis of our previous study (Chapter 2 ) examining plateletTLR9, we hypothesized that some or all of these platelet-TLRs (TLR1, 2, 4 and 6) will increase in AMI and that platelets will become activated in response to TLR stimulation (TLR4, TLR2/1, TLR2/6) in AMI subjects treated with DAPT. This brings us to the aims of this study, which are:

1. To examine whether total protein expression of platelet-TLR1, 2, 4 and 6 is different between healthy subjects and AMI subjects treated with DAPT.

2. To compare the level of platelet activation in response to TLR4, TLR2/1 and TLR2/6 agonism in both cohorts. 


\subsection{Materials and methods.}

\subsubsection{Subject recruitment.}

We recruited 12 healthy subjects and 12 AMI subjects to investigate platelet-TLR1, 2, 4 and 6 expression by western blotting. Additionally, 5 healthy and $5 \mathrm{AMI}$ subjects were recruited to examine platelet activation in response to TLR2/1, TLR4 and TLR2/6 agonism by flow cytometry. Recruitment of each of these cohorts was conducted as explained in Chapter 2 (Section 2.2.1). AMI was defined according to the Third Universal Definition of Myocardial Infarction [247]. DAPT consisted of either a combination of aspirin and clopidogrel or aspirin and ticagrelor, as described in Chapter 2. All healthy and AMI subjects provided written informed consent. Demographic data, clinical characteristics and medications were collected for all subjects at the time of enrolment. Blood was collected from a peripheral vein for all healthy subjects. For AMI subjects, blood was collected in the cardiac catheterization laboratory from the arterial sheath immediately after insertion and before administration of heparin. Blood was drawn into tubes anticoagulated with hirudin (Dynabyte).

\subsubsection{Whole platelet lysate preparation for western blotting.}

Hirudin-anticoagulated WB was centrifuged at $200 \times \mathrm{g}$ for 12 minutes to produce PRP. Prostaglandin E1 (final concentration, $1 \mu \mathrm{M}$ ) was added to PRP, which was mixed and centrifuged at $100 \times$ g for 20 minutes to deplete leukocytes. Leukocyte-depleted PRP was centrifuged at 1,000 x g for 20 minutes to pellet platelets. The platelet pellet was resuspended in radioimmunoprecipitation buffer with $10 \mu \mathrm{L} / \mathrm{mL}$ protease inhibitor cocktail (Sigma Aldrich) for 60 minutes at $4^{\circ} \mathrm{C}$. Cell debris was removed from the whole platelet lysate by centrifugation at 14,000 $\mathrm{x} g$ for 5 minutes. The protein concentration of each lysate was assessed with the bicinchoninic acid assay (Thermo Fisher Scientific) and all lysates were stored at $-80^{\circ} \mathrm{C}$ prior to western blot analysis. 


\subsubsection{Platelet-TLR expression by western blotting.}

Western blotting was used to assess expression of TLR1, 2, 4 and 6 in whole platelet lysates from healthy $(n=12)$ and AMI subjects $(n=12)$. TLR1, 2, 4 and 6 were chosen for this study as these are known to be expressed on and within healthy platelets [185, 187, 188], although there is little information on how expression of these TLRs changes during AMI. Western blotting was carried out as described in Chapter 2 (Section 2.2.4). Briefly, each whole platelet lysate was incubated with Laemmli buffer with $10 \% \beta$-mercaptoethanol and boiled at $95^{\circ} \mathrm{C}$ for 5 minutes. $50 \mu$ g of lysate was loaded onto an $8 \%$ SDS-PAGE and run at $120 \mathrm{~V}$ for 120 minutes at room temperature in running buffer. Platelet proteins were blotted onto a PVDF membrane at $20 \mathrm{~V}$ for 17 hours at $4^{\circ} \mathrm{C}$ in transfer buffer in a wet transfer system. Following this, the membrane was blocked with 5\% BSA in Trisbuffered saline supplemented with $0.1 \%$ Tween 20 for 1 hour at room temperature. The membrane was probed with either primary mouse anti-human TLR1 (clone GD2.F4; BioLegend), TLR2 (clone TL2.1; BioLegend), TLR4 (clone HTA125; BioLegend) or TLR6 (clone TLR6.127; BioLegend) overnight at $4^{\circ} \mathrm{C}$. The membrane was washed and incubated with a secondary goat anti-mouse antibody conjugated to HRP (Santa Cruz Biotechnology) and detected using an enhanced-chemiluminescent detection system (Bio Rad). Each membrane was then stripped as per manufacturer's instructions (Restore Stripping Buffer, Thermo Fisher Scientific) and probed with primary mouse anti-human $\beta$-actin (clone AC-74; Sigma Aldrich) for 2 hours at room temperature. $\beta$-actin was used as a loading control. Membranes were imaged using an Amersham Imager 600 (GE Healthcare, Little Chalfont, United Kingdom), the density of the platelet-TLR bands were analysed using ImageJ and expressed relative to the density of the corresponding $\beta$-actin band. Representative western blots for each TLR examined are presented in Figure 3.1 alongside the $\beta$-actin loading control. 


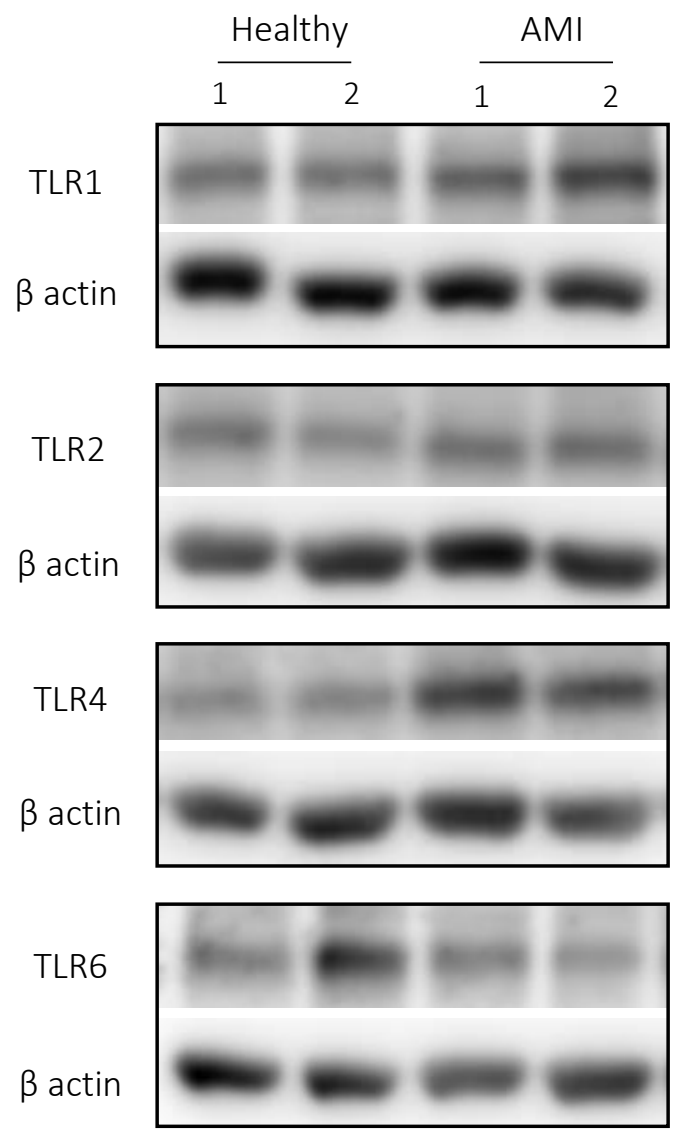

Figure 3.1 Representative examples of TLR1, 2, 4 and 6 expression in resting platelet lysates are shown for healthy ( $n=2)$ and AMI $(n=2)$ subjects. 


\subsubsection{TLR-mediated platelet activation by flow cytometry.}

Flow cytometry was used to assess platelet activation in response to TLR4, TLR2/1 and TLR2/6 agonism in WB and PRP in both healthy $(n=5)$ and AMI subjects $(n=5)$. Incubation of these TLR agonists in PRP determines the component of platelet activation that is mediated by direct TLR agonism. Incubation in WB determines whether any component of platelet activation is mediated by indirect TLR agonism through 'off-platelet' TLR pathways. Following blood drawing, leukocytedepleted PRP was prepared. Both WB and PRP were adjusted to $1 \times 10^{8}$ platelets $/ \mathrm{mL}$ with PBS. WB and PRP were incubated for 15 minutes at room temperature with $0.1-100 \mu \mathrm{g} / \mathrm{mL}$ of the following TLR agonists: lipopolysaccharide (LPS) from Escherichia coli serotype R515 (TLR4 agonist; Enzo Life Sciences, Farmingdale, NY, USA), Pam3CSK4 (TLR2/1 agonist; Tocris Bioscience, Bristol, UK), and fibroblast-stimulating lipopeptide (FSL)-1 (TLR2/6 agonist; Santa Cruz Biotechnology). As a positive control, PRP and WB were incubated with $2.5 \mu \mathrm{M}$ and $10 \mu \mathrm{M}$ TRAP6 (Tocris Bioscience) for 15 minutes at room temperature. As a negative control, PRP and WB were incubated with PBS.

Following incubation with these TLR agonists, PRP and WB were incubated with anti-CD42a-PerCP (clone Beb-1; Becton Dickinson), anti-CD62p-PE (clone AK4; BioLegend) and PAC1-FITC (clone PAC1; Becton Dickinson) in staining buffer for 20 minutes in the dark at room temperature (see Table 3.1 for panel design). In parallel, aliquots of PRP and WB were incubated with PerCP-mouse IgG1,k (Becton Dickinson), PE-mouse IgG1,k (BioLegend) or FITC-mouse IgM (Becton Dickinson) isotype controls. Antibody-stained PRP and WB were fixed with $1 \%$ paraformaldehyde and analysed on a Millipore Guava easyCyte 8HT flow cytometer (Merck Millipore). Flow cytometric data was analysed using FlowJo software (v10.0.7, Tree star). Platelets were identified, firstly, by their

forward and side scatter properties and, secondly, by their CD42a-positivity (Figure 3.2). The percentage of CD42a-positive platelets that were positive for CD62p and PAC1 was determined, as a distinct positive and negative platelet population for each marker was observed. 
Representative examples of CD62p and PAC1 expression in the platelet population are given in Figure 3.3.

Table 3.1 Panel design for assessing platelet activation in response to TLR agonism.

\begin{tabular}{|l|l|l|l|}
\hline & $\begin{array}{l}\text { Laser/channel } \\
\text { (fluorophore) }\end{array}$ & $\begin{array}{l}\text { Antibody target } \\
\text { (clone) }\end{array}$ & $\begin{array}{l}\text { Concentration in final staining } \\
\text { volume (final dilution) }\end{array}$ \\
\hline TLR-mediated platelet & Blue/Red (PerCP) & CD42a (Beb1) & $1.25 \mu \mathrm{g} / \mathrm{mL}(1$ in 10) \\
activation & Blue/Yellow (PE) & CD62p (AK-4) & $1.00 \mu \mathrm{g} / \mathrm{mL}(1$ in 100) \\
& Blue/Green (FITC) & PAC1 (PAC1) & $1.00 \mu \mathrm{g} / \mathrm{mL}(1 \mathrm{in} \mathrm{20)}$ \\
\hline
\end{tabular}

A list of all antibody formats, clones, isotypes and manufacturers can be found in Appendix 1. 

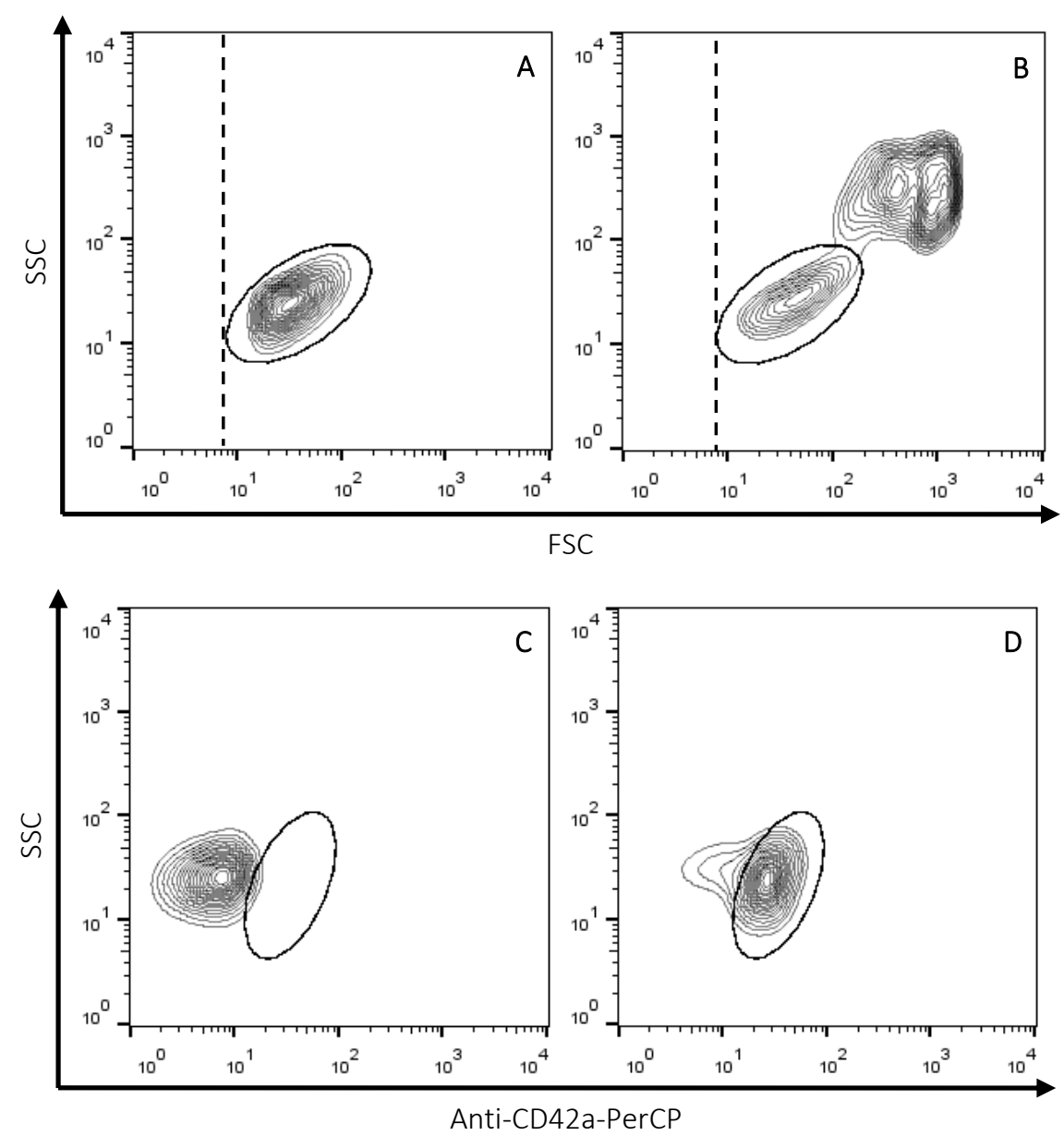

Figure 3.2 Platelets were identified by flow cytometry.

The platelet population was identified from PRP (A) and WB (B) by their forward and side scatter properties. Platelets were further identified by their CD42a-positivity (D) against the isotype control (C). 

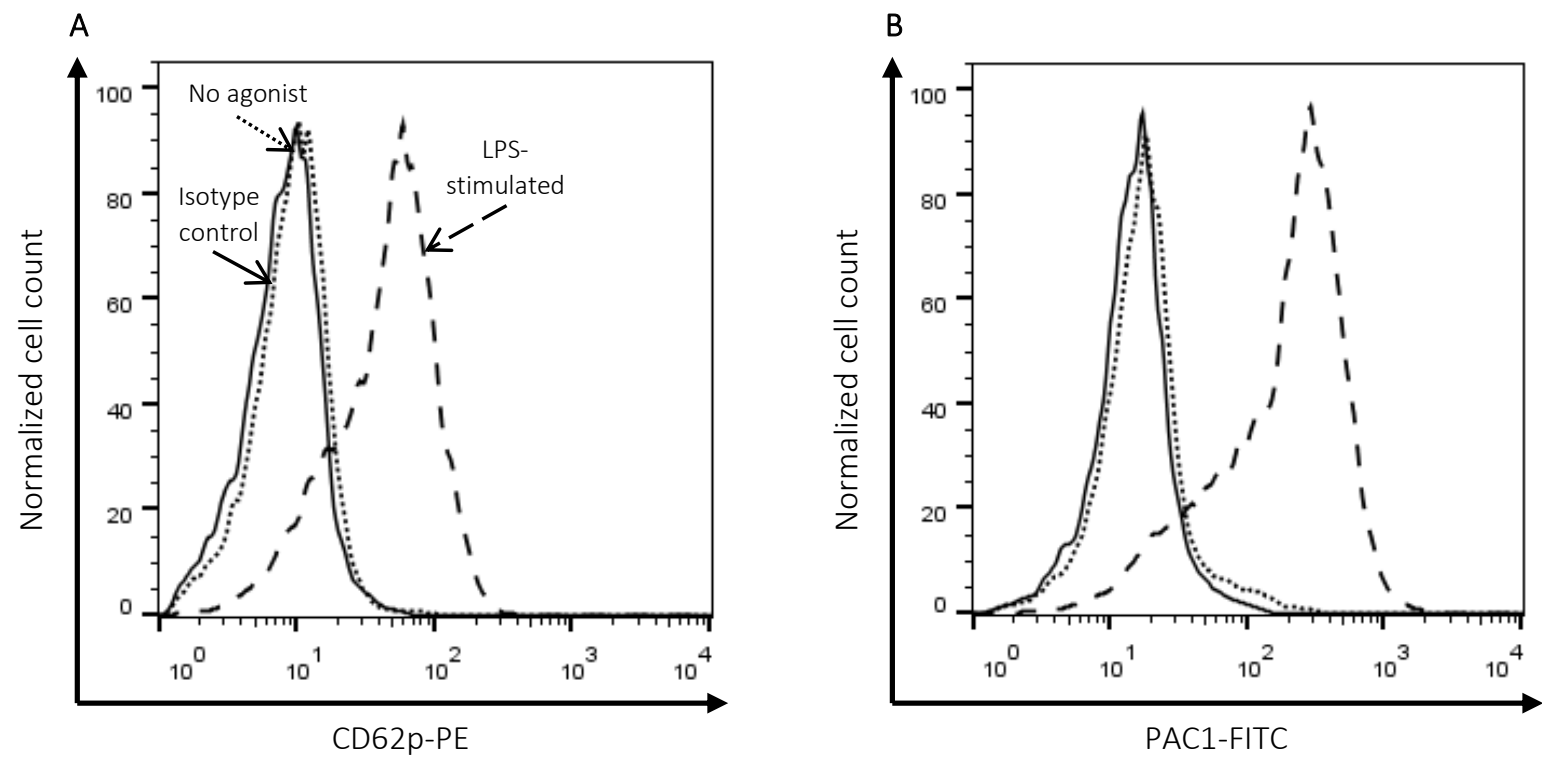

Figure 3.3 Representative plots of platelet activation markers.

A representative plot of CD62p expression (A) and PAC1 expression (B) is shown for unstimulated and LPS-stimulated $(100 \mu \mathrm{g} / \mathrm{mL}) \mathrm{CD} 42 \mathrm{a}+$ platelets. Solid line, isotype control; dotted line, no agonist; dashed line, LPS stimulated. 


\subsubsection{TLR-mediated platelet-monocyte aggregation (PMAgg) by flow cytometry.}

WB was incubated for 15 minutes at room temperature with the following: PBS, $2.5 \mu \mathrm{M}$ TRAP, 10 $\mu \mathrm{M}$ TRAP, $0.1-100 \mu \mathrm{g} / \mathrm{mL}$ of Pam3CSK4, LPS and FSL-1. Following incubation, WB was incubated with anti-CD42a-PerCP (Becton Dickinson) and anti-CD64-APC/Cy7 (clone 10.1; BioLegend) in staining buffer for 20 minutes in the dark at room temperature (Table 3.2). PerCP-mouse IgG1,k (Becton Dickinson) and APC/Cy7-mouse IgG1,k (BioLegend) isotype controls were run in parallel. Following antibody staining, WB was diluted with red blood cell lysis buffer (Qiagen, Hilden, Germany), incubated for 15 minutes and centrifuged at $200 \times$ g for 5 minutes. The supernatant was removed and the cell pellet was washed in lysis buffer again before resuspension in $1 \%$ paraformaldehyde and flow cytometric analysis. Flow cytometric data was analysed using FlowJo software (Treestar). Leukocytes were identified by their forward and side scatter properties and monocytes were identified by CD64 expression (Figure 3.4). Platelet-monocyte aggregation (PMAgg) was analysed by changes in MFI of the platelet-specific marker, CD42a, following gating on CD64+ monocytes (Figure 3.4).

Table 3.2 Panel design for assessing platelet-monocyte aggregation following TLR agonism.

\begin{tabular}{|l|l|l|l|}
\hline & $\begin{array}{l}\text { Laser/channel } \\
\text { (fluorophore) }\end{array}$ & $\begin{array}{l}\text { Antibody target } \\
\text { (clone) }\end{array}$ & $\begin{array}{l}\text { Concentration in final staining } \\
\text { volume (final dilution) }\end{array}$ \\
\hline Platelet-monocyte & Blue/Red (PerCP) & CD42a (Beb1) & $1.25 \mu \mathrm{g} / \mathrm{mL}(1 \mathrm{in} \mathrm{10)}$ \\
aggregates & Red/NIR2 (APC/Cy7) & CD64 (10.1) & $1.00 \mu \mathrm{g} / \mathrm{mL}(1 \mathrm{in} \mathrm{200)}$ \\
\hline
\end{tabular}

A list of all antibody formats, clones, isotypes and manufacturers can be found in Appendix 1. 


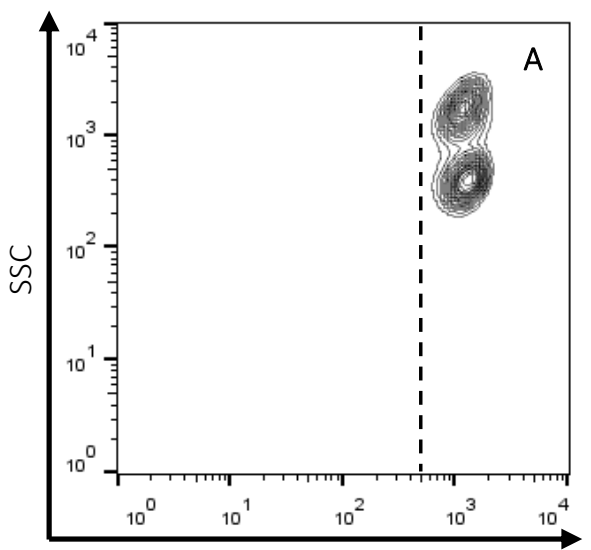

FSC

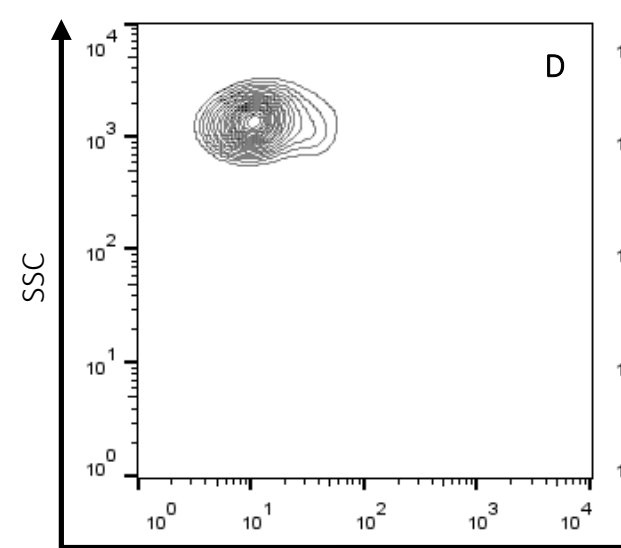

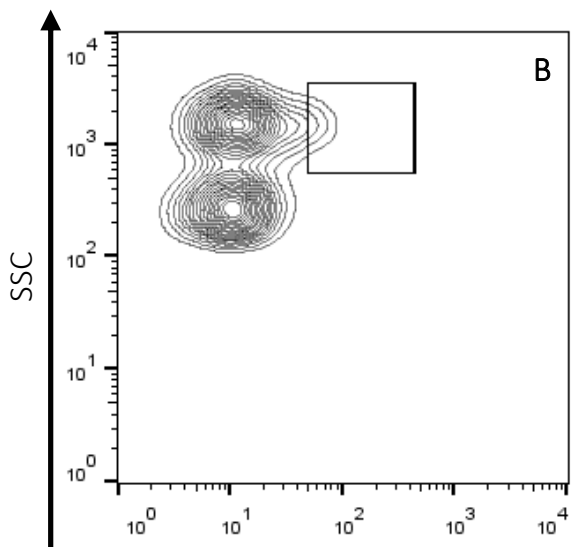

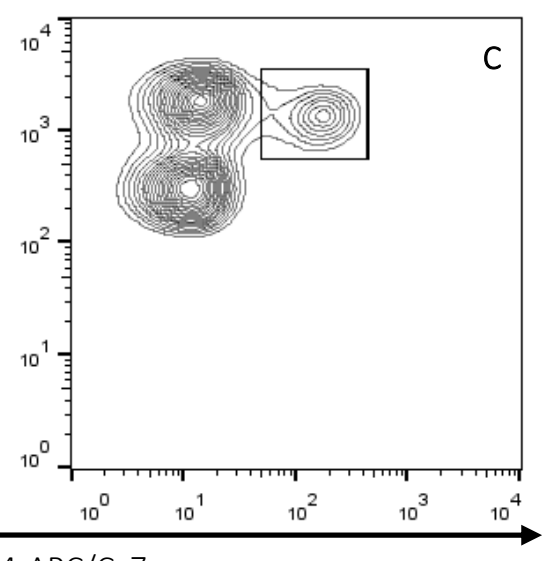

Anti-CD64-APC/Cy7
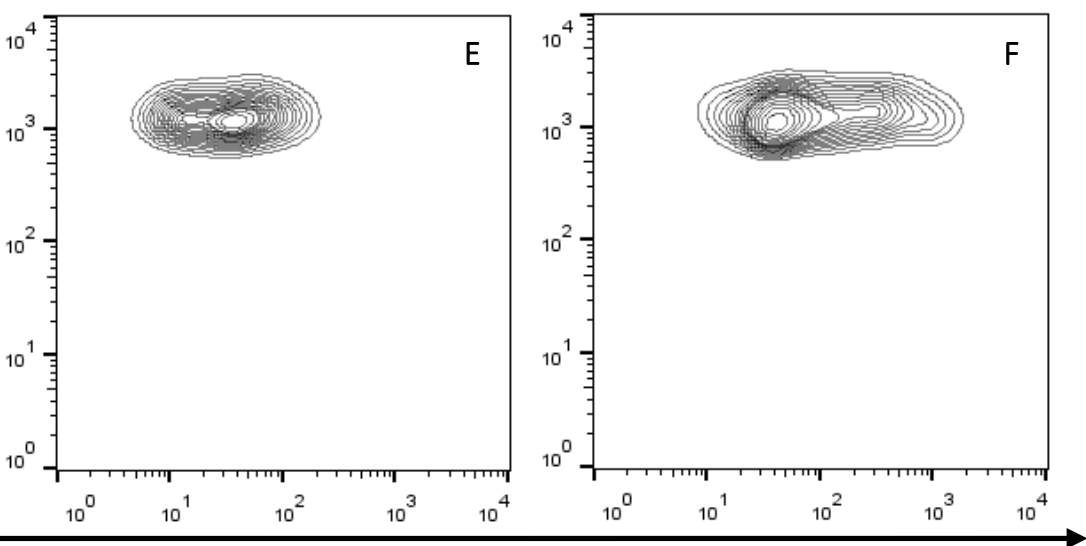

Anti-CD42a-PerCP

Figure 3.4 Platelet monocyte aggregation was measured by flow cytometry.

Leukocytes were identified by their forward and side scatter properties (A). Monocytes were identified by CD64 expression (C) against the isotype control (B). Platelet-monocyte aggregation (PMAgg) was analysed as the change in MFI of CD42a, the platelet-specific marker, of gated CD64+ monocytes. A representative plot of PMAgg is shown for

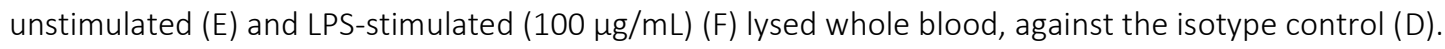




\subsubsection{TLR-mediated monocyte activation by flow cytometry.}

Activated (CD63-positive) monocytes were identified in response to overnight LPS and FSL-1 stimulation to verify that these agonists are able to activate leukocytes. EDTA-anticoagulated WB drawn from 5 healthy subjects was diluted 1:1 with PBS, layered over Histopaque-1077 and centrifuged at $400 \times \mathrm{g} / 30$ minutes. The peripheral blood mononuclear cell (PBMC) layer was isolated, diluted in PBS and centrifuged at $100 \mathrm{xg} / 20$ minutes. The platelet-containing supernatant was discarded, the PBMC pellet was washed in PBS and resuspended in cell culture media (10\% FCS, 2 mM L-glutamate, $100 \mathrm{U} / \mathrm{mL}$ penicillin, $100 \mu \mathrm{g} / \mathrm{mL}$ streptomycin, 0.01M HEPES buffer, 0.1\% $\beta$-mercaptoethanol, $0.01 \mathrm{nM}$ non-essential amino acids). PBMCs were plated at a concentration of $1 \times 10^{6}$ cells $/ \mathrm{mL}$ and primed for 3 hours at $37^{\circ} \mathrm{C} / 5 \% \mathrm{CO}_{2}$ with $10 \mathrm{ng} / \mathrm{mL}$ human IFN- $\gamma$. Following priming, LPS or FSL-1 was added to a final concentration of 0.01 to $100 \mathrm{ng} / \mathrm{mL}$, and PBMCs were incubated for a further 24 hours at $37^{\circ} \mathrm{C} / 5 \% \mathrm{CO}_{2}$. PBMCs were then centrifuged at $400 \times \mathrm{g}$ for 10 minutes, washed with PBS and resuspended in staining buffer. PBMCs were incubated with antiCD64-APC/Cy7 (clone 10.1; BioLegend) and anti-CD63-AF647 (clone H5C6; BioLegend) for 20 minutes at RT (see Table 3.3 for panel design). In parallel, PBMCs were incubated with APC/Cy7IgG1,k or AF647-IgG1,k (BioLegend). All samples were fixed with 1\% paraformaldehyde before analysis on a Guava easyCyte 8HT flow cytometer (Merck Millipore). The monocyte population was identified by forward and side scatter followed by CD64 expression as seen in Figure 3.4. For CD63 expression, the MFI was determined, and representative plots of CD63 expression is shown in Figure 3.5. Flow cytometric data was analysed using FlowJo software (Tree star).

Table 3.3 Panel design for assessing monocyte activation in response to TLR agonism.

\begin{tabular}{|l|l|l|l|}
\hline & $\begin{array}{l}\text { Laser/channel } \\
\text { (fluorophore) }\end{array}$ & $\begin{array}{l}\text { Antibody target } \\
\text { (clone) }\end{array}$ & $\begin{array}{l}\text { Concentration in final staining } \\
\text { volume (final dilution) }\end{array}$ \\
\hline Monocyte activation & Red/Red2 (AF647) & CD63 (H5C6) & $1.00 \mu \mathrm{g} / \mathrm{mL}(1: 200)$ \\
& Red/NIR2 (APC/Cy7) & CD64 (10.1) & $1.00 \mu \mathrm{g} / \mathrm{mL}(1: 200)$ \\
\hline
\end{tabular}

A list of all antibody formats, clones, isotypes and manufacturers can be found in Appendix 1. 


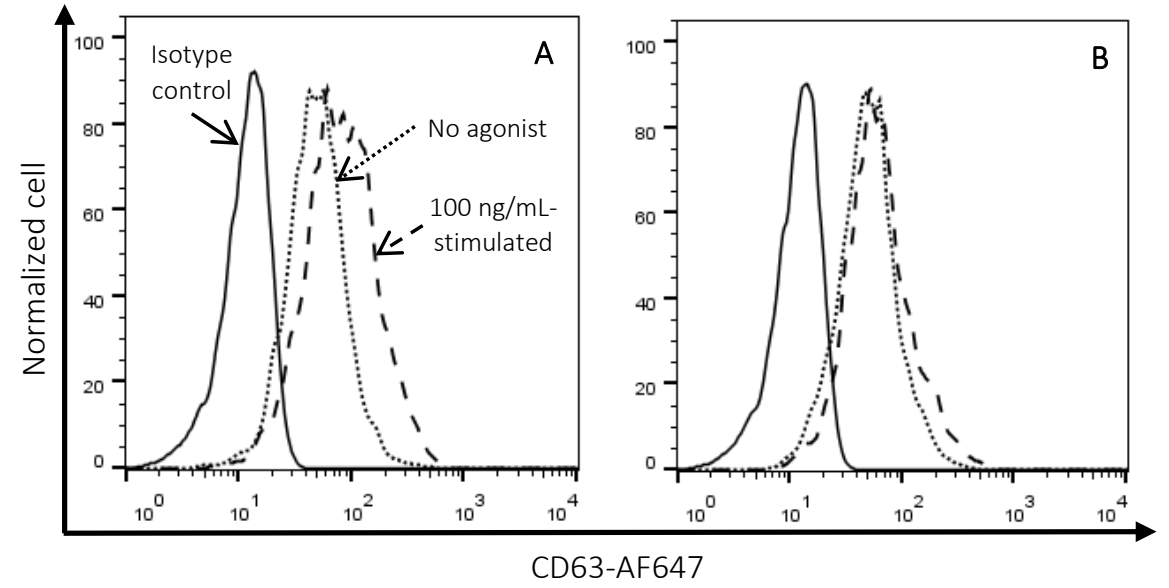

\section{Figure 3.5 Representative plots of monocyte CD63 expression.}

Representative plots of CD63 expression is shown for unstimulated, LPS-stimulated (A) and FSL-1-stimulated (B) CD64+ monocytes. Solid line, isotype control; dotted line, no agonist; dashed line, $100 \mathrm{ng} / \mathrm{mL}$-stimulated. 


\subsubsection{Statistical analysis.}

With a sample size of 12 , we have the power to detect a $30 \%$ difference in platelet-TLR expression between healthy and AMI subjects with $80 \%$ power and $5 \%$ significance. With a sample size of 5 , we have the power to detect a $20 \%$ difference in platelet activation between the unstimulated platelet sample and the TLR-stimulated platelet sample with $80 \%$ power and 5\% significance. These calculations were based on the mean and standard deviation of platelet-TLR expression and TLR-mediated platelet activation seen in healthy and AMI subjects in Chapter 2.

For demographic data, categorical variables are expressed as frequencies and percentages. Continuous variables are expressed as mean \pm SD. Continuous variables were analysed with unpaired t-tests and categorical variables were analysed using Chi-square test. Differences between cohorts were examined using unpaired t-tests. Differences in responses to each TLR agonist within cohorts were examined using one-way ANOVA with post-hoc Tukey's multiple comparisons tests. All statistical analyses were performed using GraphPad Prism 6 (GraphPad Software Inc., La Jolla, CA, USA). 


\subsection{Results.}

\subsubsection{TLR expression in healthy and AMI platelets.}

The demographic details of the healthy $(n=12)$ and AMI $(n=12)$ subjects recruited to examine platelet expression of TLR1, 2, 4 and 6 are given in Table 3.4. TLR expression was examined by western blotting and expressed as a ratio of the corresponding $\beta$-actin expression. Expression of each TLR in the healthy cohort was normalized to 1 , and TLR expression in the AMI cohort was compared to this normalized response. For TLR1 and TLR4, protein expression in AMI platelets was significantly greater than in healthy platelets (Figure 3.6). TLR1 expression was 53\% greater (1.00 \pm 0.39 vs. $1.53 \pm 0.48, p<0.01)$ and TLR4 expression was $58 \%$ greater $(1.00 \pm 0.41$ vs. $1.58 \pm 0.59$, $\mathrm{p}=0.011)$ in AMI platelets. TLR2 expression (1.00 \pm 0.35 vs. $1.23 \pm 0.46, p=0.18)$ and TLR6 expression ( $1.00 \pm 0.60$ vs. $0.95 \pm 0.65, p=0.85)$ were not statistically significantly different between cohorts.

Interestingly, we describe a bimodal pattern of expression in the AMI cohort for TLR1, 4 and 6 (Figure 3.7). A group of AMI subjects cluster above (TLR1, $n=6,1.93 \pm 0.32$; TLR4, $n=5,2.16 \pm 0.45$; TLR6, $n=4,1.79 \pm 0.28$ ) and below (TLR1, $n=6,1.13 \pm 0.13 ;$ TLR4, $n=7,1.16 \pm 0.17 ; T L R 6, n=8$, $0.53 \pm 0.19)$ the mean of the AMI cohort. 
Table 3.4 Demographics and clinical characteristics of each cohort for investigating TLR expression and TLR-mediated platelet activation.

\begin{tabular}{|c|c|c|c|c|c|c|}
\hline & \multicolumn{3}{|c|}{ Platelet-TLR expression } & \multicolumn{3}{|c|}{ TLR-mediated platelet activation } \\
\hline & AMI cohort $(n=12)$ & Healthy cohort $(n=12)$ & $p$-value & AMI cohort $(n=5)$ & Healthy cohort $(n=5)$ & $p$-value \\
\hline \multicolumn{7}{|l|}{ Demographics } \\
\hline Age, years & $57 \pm 8$ & $55 \pm 8$ & $p=0.65$ & $56 \pm 17$ & $48 \pm 4$ & $p=0.37$ \\
\hline Male & $8(67)$ & $8(67)$ & -- & $8(67)$ & $8(67)$ & -- \\
\hline $\mathrm{BMI}, \mathrm{kg} / \mathrm{m}^{2}$ & $29 \pm 5$ & $23 \pm 2$ & $p<0.01$ & $27 \pm 3$ & $26 \pm 4$ & $p=0.48$ \\
\hline \multicolumn{7}{|c|}{ Cardiovascular risk factors } \\
\hline Hypertension & $6(50)$ & $0(0)$ & -- & $2(40)$ & $0(0)$ & -- \\
\hline Dyslipidaemia & $9(75)$ & $0(0)$ & -- & $3(60)$ & $0(0)$ & -- \\
\hline Diabetes & $0(0)$ & $0(0)$ & -- & $1(20)$ & $0(0)$ & -- \\
\hline Current smoker & $1(8)$ & $0(0)$ & -- & $2(40)$ & $0(0)$ & -- \\
\hline Renal dysfunction & $0(0)$ & $0(0)$ & -- & $0(0)$ & $0(0)$ & -- \\
\hline \multicolumn{7}{|c|}{ Clinical presentation } \\
\hline STEMI & $6(50)$ & $0(0)$ & -- & $1(20)$ & $0(0)$ & -- \\
\hline NSTEMI & $6(50)$ & $0(0)$ & -- & $4(80)$ & $0(0)$ & -- \\
\hline \multicolumn{7}{|c|}{ Anti-platelet medication } \\
\hline Aspirin & $12(100)$ & $0(0)$ & -- & $5(100)$ & $0(0)$ & -- \\
\hline Clopidogrel & $10(83)$ & $0(0)$ & -- & $1(20)$ & $0(0)$ & -- \\
\hline Ticagrelor & $2(17)$ & $0(0)$ & -- & $4(80)$ & $0(0)$ & -- \\
\hline
\end{tabular}

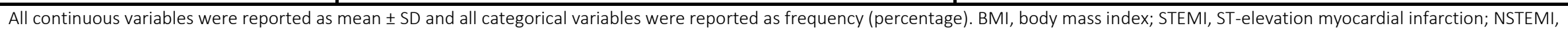
non-ST-elevation myocardial infarction. 


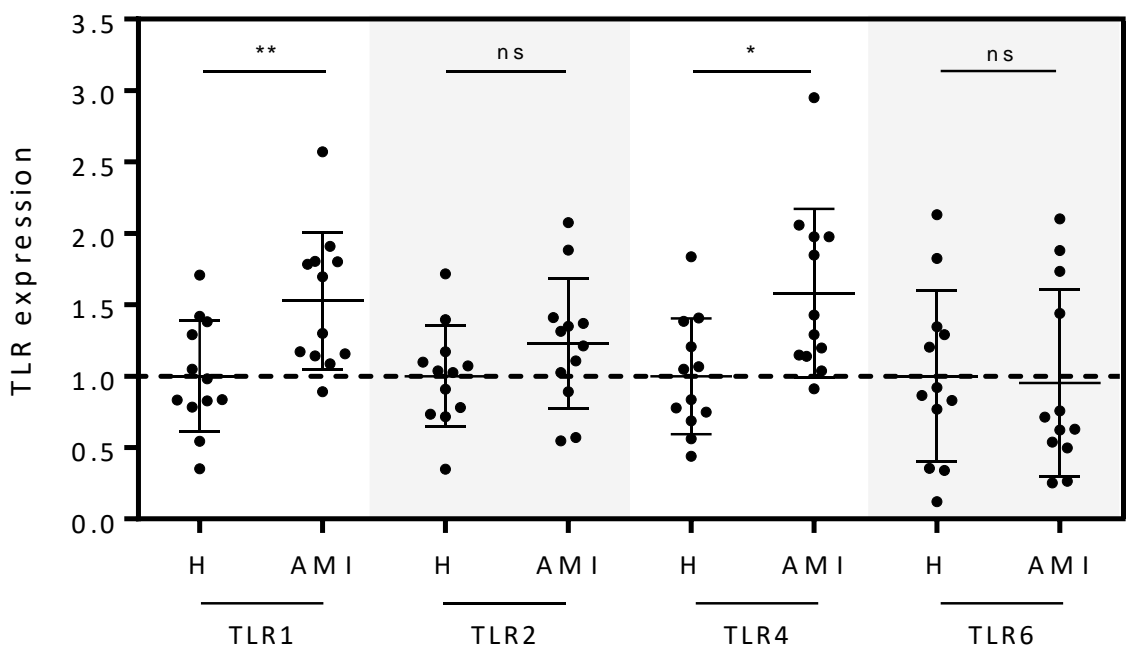

Figure 3.6 AMI platelets express higher levels of TLR1 and TLR4, but not TLR2 or TLR6, compared to healthy platelets.

TLR expression in resting platelet lysates was analysed in the healthy $(\mathrm{H})$ and $\mathrm{AMI}$ cohort. Platelet-TLR expression was measured as a ratio of the corresponding $\beta$-actin expression signal. The ratio was normalized to a response of 1 in the healthy cohort (dotted line) and the expression of TLRs for each AMI subject was expressed against this normalized response of 1 . Mean \pm SD for each cohort are shown, unpaired t-tests were performed to detect differences, $* p<0.05$, $* * \mathrm{p}<0.01$.

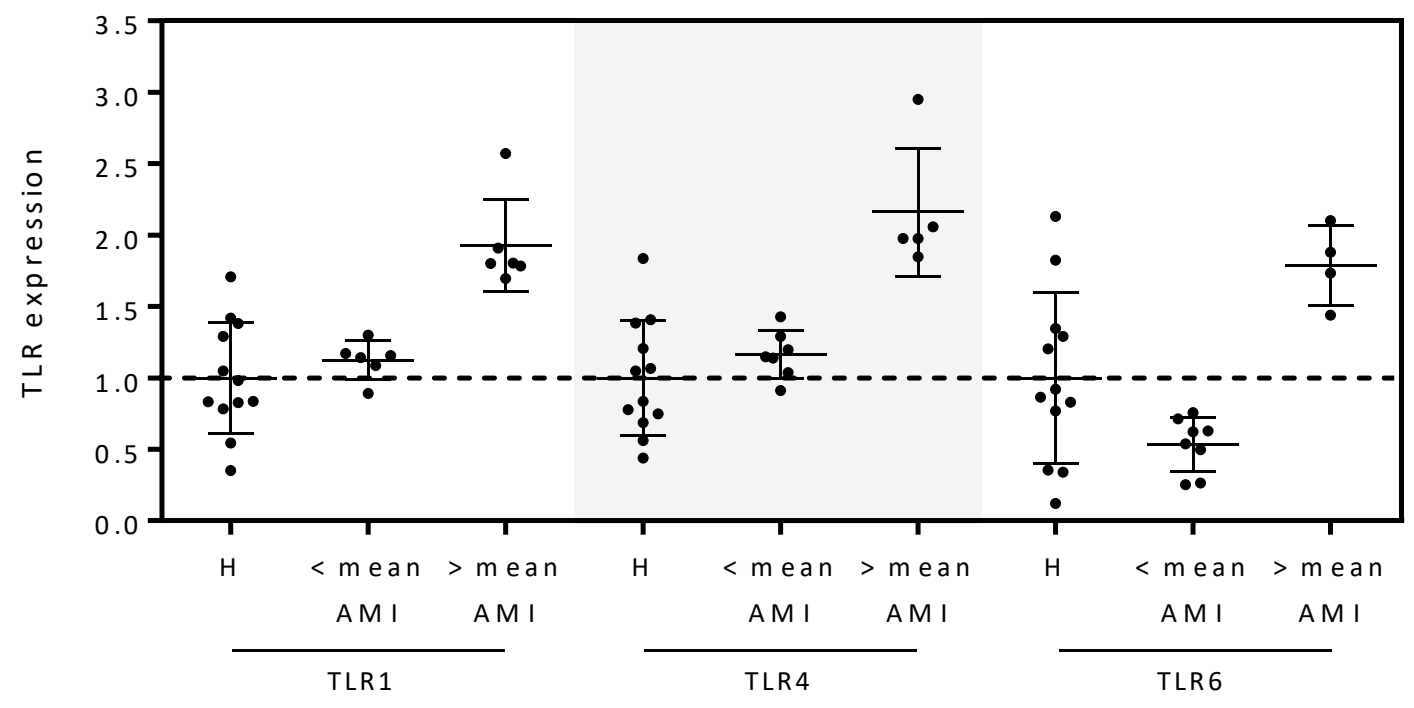

Figure 3.7 TLR1, 4 and 6 expression shows a bimodal distribution in the AMI cohort.

Platelet-TLR expression was examined in the healthy $(H)$ and AMI cohort. Some AMI subjects have higher TLR1, 4 and 6 expression (>mean) than other AMI subjects (<mean). Mean \pm SD for 12 subjects in each cohort are shown. 


\subsubsection{TLR-mediated platelet activation in healthy and AMI platelets.}

Platelet activation in response to TLR agonism was examined in 5 healthy and $5 \mathrm{AMI}$ subjects (for demographic data, see Table 3.4). PRP and WB from each subject were incubated with $0.1-100$ $\mu \mathrm{g} / \mathrm{mL}$ of LPS (TLR4 agonist), Pam3CSK4 (TLR2/1 agonist) and FSL-1 (TLR2/6 agonist) and platelet activation was assessed by surface expression of CD62p and PAC1. TLR agonism in PRP assesses direct platelet activation and, in WB, TLR agonism assesses any additional indirect platelet activation.

\subsubsection{Platelet activation in response to LPS stimulation.}

In response to LPS, increased expression of both CD62p and PAC1 was only seen following incubation with the highest dose $(100 \mu \mathrm{g} / \mathrm{mL})$ in both the healthy and AMI cohorts. In PRP, $67.6 \pm$ $16.7 \%$ of the platelet population was CD62p-positive in response to high-dose LPS in healthy subjects (Figure 3.8A) and $57.6 \pm 19.2 \%$ of platelets were CD62p-positive in AMI subjects (Figure 3.8B). For both cohorts, these expression levels were significantly higher than in unstimulated platelets (Healthy, $2.9 \pm 3.4 \%$; AMI, $0.5 \pm 2.1 \%$; both p<0.05). For both cohorts, CD62p expression in response to $100 \mu \mathrm{g} / \mathrm{mL}$ LPS in WB did not differ significantly from PRP. These results in WB are presented in Figure 3.9. All results for CD62p positivity are given in Table 3.5. Across cohorts, similar patterns of platelet activation in response to high-dose LPS were observed for PAC1 expression in PRP (Figure 3.10) and WB (Figure 3.11). All results for PAC1 positivity are given in Table 3.6. Healthy and AMI platelets were non-responsive to any other dose of LPS across both WB and PRP. 
Healthy platelet-rich plasma
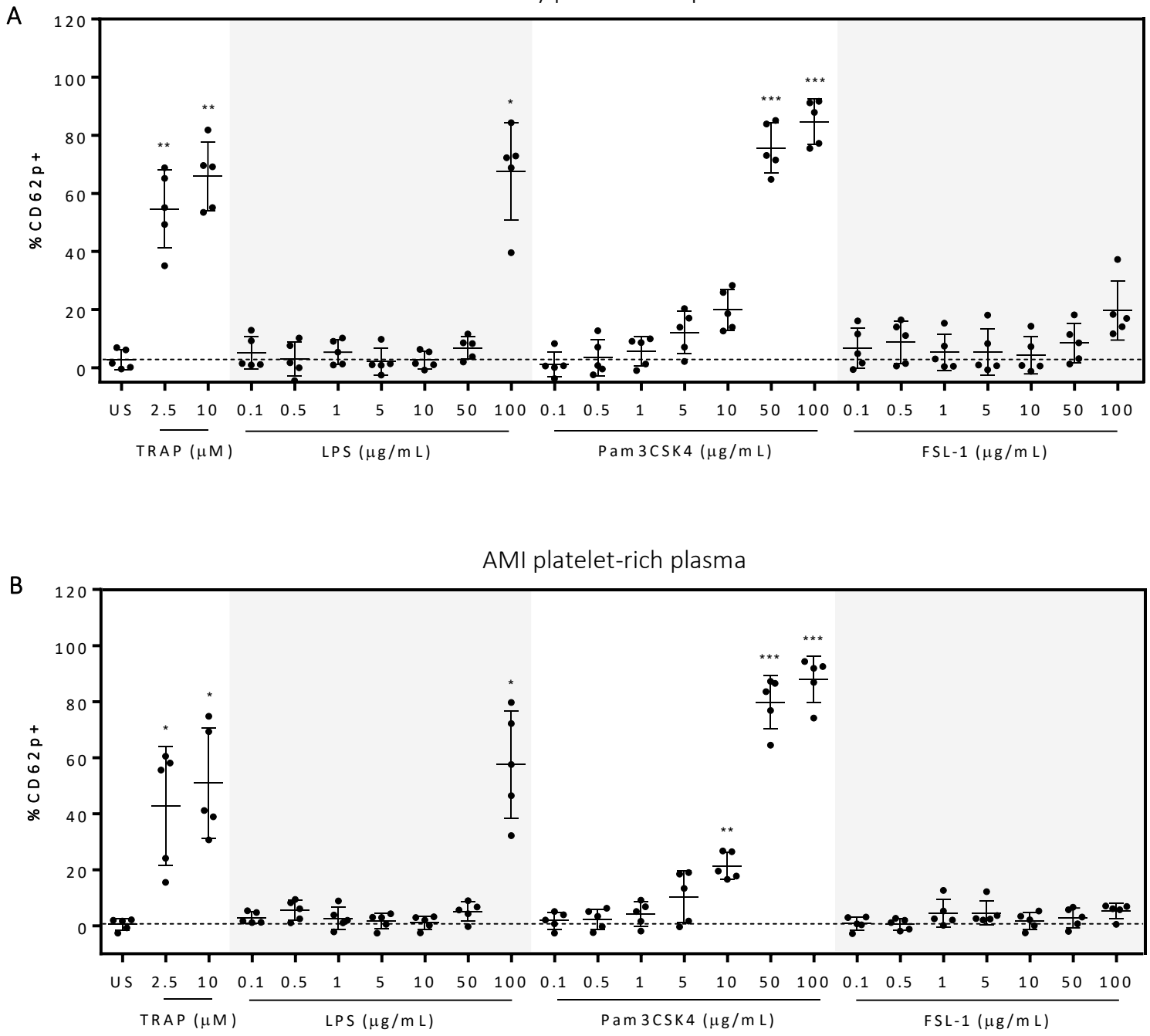

Figure 3.8 Platelets upregulate CD62p in response to TLR agonism in PRP.

Platelet activation, as measured by CD62p positivity, was assessed in PRP in 5 healthy (A) and 5 AMI subjects (B). Within each cohort, one-way ANOVA was found to be significant $(p<0.05)$ for each parameter and Tukey's multiple comparisons test was performed to detect differences. * indicates that the value for the treatment is significantly different than the unstimulated sample. Mean \pm SD for 5 subjects for each cohort is shown. ${ }^{*} p<0.05, * * p<0.01, * * *$ $p<0.001$. The dotted line marks the mean level of platelet activation in unstimulated platelets. 
Healthy whole blood

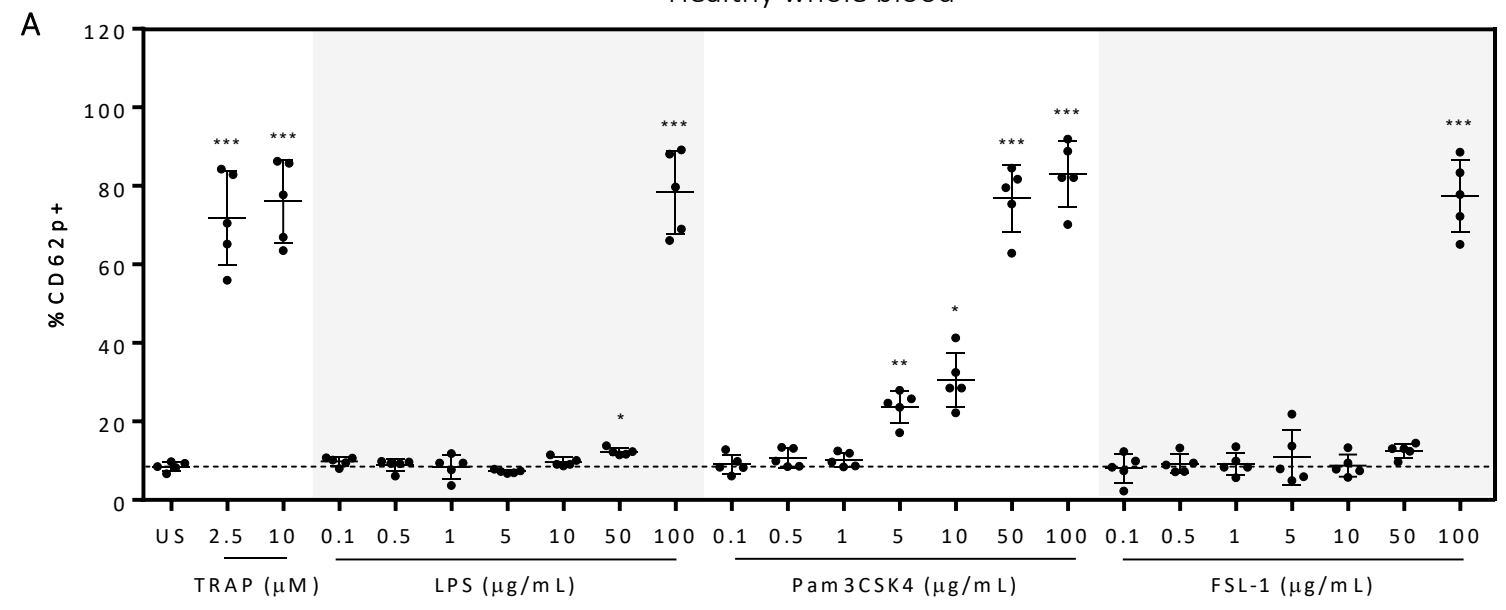

AMI whole blood

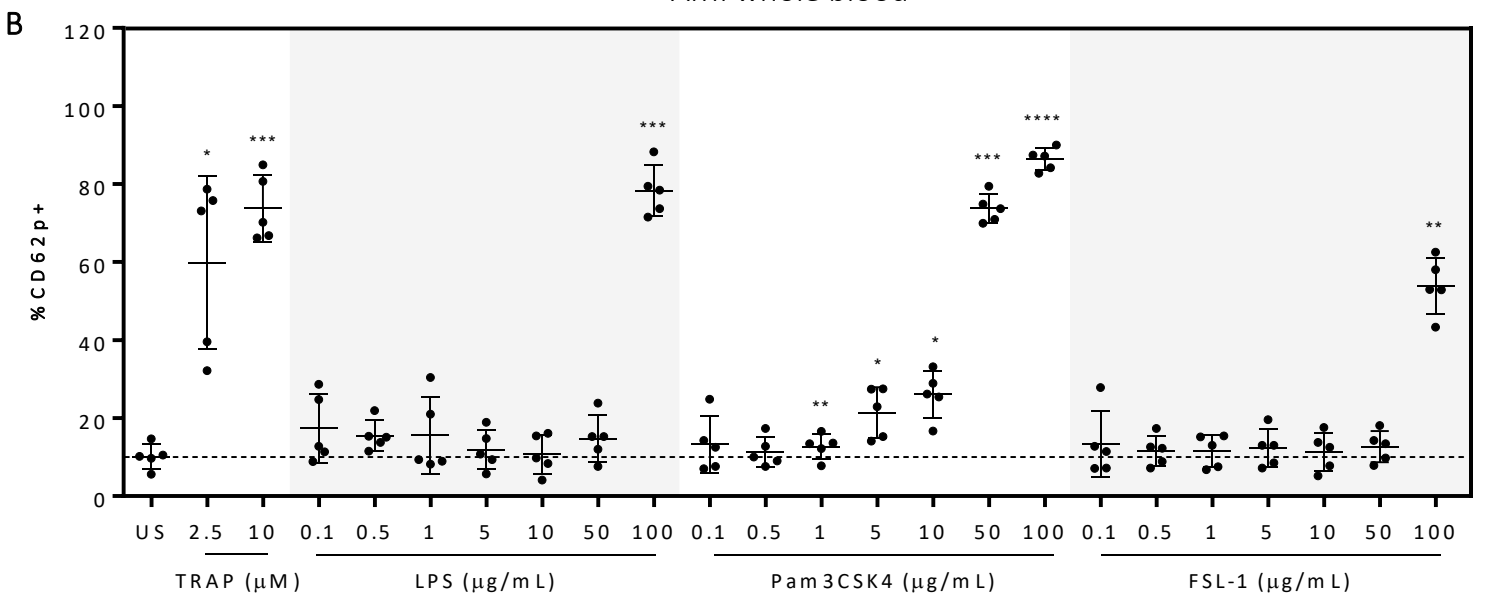

Figure 3.9 Platelets express CD62p in response to TLR agonism in WB.

CD62p-positivity was assessed in 5 healthy (A) and 5 AMI subjects (B). Within each cohort, one-way ANOVA was found to be significant $(p<0.05)$ for each parameter and Tukey's multiple comparisons test was performed to detect differences. * indicates that the value for the treatment is significantly different than the unstimulated sample. Mean \pm SD for 5 subjects in each cohort is shown. ${ }^{*} p<0.05, * * p<0.01, * * * p<0.001, * * * * p<0.0001$. The dotted line marks the mean level of platelet activation in unstimulated platelets. 
Table 3.5 Incubation with a range of TLR agonists induces variable CD62p-positivity in the healthy and AMI cohorts.

\begin{tabular}{|c|c|c|c|c|c|}
\hline & & \multicolumn{2}{|c|}{ Healthy } & \multicolumn{2}{|c|}{ AMI } \\
\hline & & WB & PRP & WB & PRP \\
\hline US & -- & $8.5 \pm 1.2$ & $2.9 \pm 3.4$ & $10.1 \pm 3.2$ & $0.5 \pm 2.1$ \\
\hline \multirow{2}{*}{ TRAP $(\mu \mathrm{M})$} & 2.5 & $71.8 \pm 12.0^{* * *}$ & $54.7 \pm 13.4^{* *}$ & $59.9 \pm 22.1^{*}$ & $42.8 \pm 21.3^{*}$ \\
\hline & 10 & $76.0 \pm 10.5^{* * *}$ & $65.9 \pm 11.7^{* *}$ & $73.8 \pm 8.5^{* * *}$ & $51.0 \pm 19.8^{*}$ \\
\hline \multirow{7}{*}{ LPS ( $\mu \mathrm{g} / \mathrm{mL})$} & 0.1 & $9.8 \pm 1.1$ & $5.1 \pm 5.6$ & $17.3 \pm 8.8$ & $2.8 \pm 2.1$ \\
\hline & 0.5 & $8.8 \pm 1.5$ & $3.0 \pm 5.9$ & $15.5 \pm 3.9$ & $5.5 \pm 3.6$ \\
\hline & 1 & $8.4 \pm 3.0$ & $5.4 \pm 4.3$ & $15.6 \pm 9.8$ & $2.7 \pm 4.1$ \\
\hline & 5 & $7.2 \pm 0.4$ & $2.1 \pm 4.6$ & $11.9 \pm 5.1$ & $1.6 \pm 2.7$ \\
\hline & 10 & $9.7 \pm 1.1$ & $2.7 \pm 3.1$ & $10.8 \pm 5.0$ & $1.2 \pm 2.7$ \\
\hline & 50 & $12.3 \pm 0.9 *$ & $6.9 \pm 3.9$ & $14.8 \pm 5.9$ & $5.1 \pm 3.4$ \\
\hline & 100 & $78.4 \pm 10.6 * * *$ & $67.6 \pm 16.7^{*}$ & $78.3 \pm 6.5^{* * *}$ & $57.6 \pm 19.2^{*}$ \\
\hline \multirow{7}{*}{$\begin{array}{l}\text { Pam3CSK4 } \\
(\mu \mathrm{g} / \mathrm{mL})\end{array}$} & 0.1 & $9.1 \pm 2.5$ & $1.2 \pm 4.4$ & $13.2 \pm 7.2$ & $13.2 \pm 7.2$ \\
\hline & 0.5 & $10.7 \pm 2.4$ & $3.5 \pm 6.2$ & $11.3 \pm 3.8$ & $11.3 \pm 3.8$ \\
\hline & 1 & $10.2 \pm 1.8$ & $5.6 \pm 5.1$ & $12.7 \pm 3.2 * *$ & $4.2 \pm 4.4$ \\
\hline & 5 & $23.8 \pm 4.1^{* *}$ & $12.1 \pm 7.4$ & $21.4 \pm 6.4^{*}$ & $10.4 \pm 9.2$ \\
\hline & 10 & $30.6 \pm 7.0 *$ & $19.9 \pm 7.0$ & $26.1 \pm 6.1^{*}$ & $21.4 \pm 4.8^{* *}$ \\
\hline & 50 & $76.8 \pm 8.5^{* * *}$ & $75.7 \pm 8.7 * * *$ & $73.8 \pm 3.7^{* * *}$ & $79.7 \pm 9.5^{* * *}$ \\
\hline & 100 & $83.0 \pm 8.4^{* * *}$ & $84.7 \pm 7.8^{* * *}$ & $86.3 \pm 2.8^{* * * *}$ & $88.0 \pm 8.2^{* * *}$ \\
\hline \multirow{7}{*}{$\begin{array}{c}\text { FSL-1 } \\
(\mu \mathrm{g} / \mathrm{mL})\end{array}$} & 0.1 & $8.1 \pm 3.7$ & $6.7 \pm 7.0$ & $13.3 \pm 8.5$ & $0.9 \pm 2.4$ \\
\hline & 0.5 & $9.2 \pm 2.4$ & $8.7 \pm 7.3$ & $11.7 \pm 3.9$ & $0.6 \pm 2.0$ \\
\hline & 1 & $9.2 \pm 2.9$ & $5.3 \pm 6.3$ & $11.6 \pm 4.2$ & $4.5 \pm 4.9$ \\
\hline & 5 & $10.8 \pm 7.0$ & $5.5 \pm 7.9$ & $12.3 \pm 4.9$ & $4.6 \pm 4.3$ \\
\hline & 10 & $8.8 \pm 1.8$ & $4.4 \pm 6.4$ & $11.4 \pm 4.9$ & $1.7 \pm 3.0$ \\
\hline & 50 & $12.5 \pm 1.8$ & $8.5 \pm 6.8$ & $12.7 \pm 4.0$ & $2.8 \pm 3.5$ \\
\hline & 100 & $77.4 \pm 9.2^{* * *}$ & $19.7 \pm 10.2$ & $53.9 \pm 7.2^{* *}$ & $5.3 \pm 2.7$ \\
\hline
\end{tabular}

Mean \pm SD for 5 subjects in each cohort is shown. Within each cohort, one-way ANOVA was found to be significant $(p<0.05)$ for each parameter and Tukey's multiple comparisons tests were performed. * indicates the value for the treatment is significantly different than the unstimulated sample. ${ }^{*} p<0.05,{ }^{* *} p<0.01,{ }^{* * *} p<0.001,{ }^{* * * *} p<0.0001$. 
Healthy platelet-rich plasma

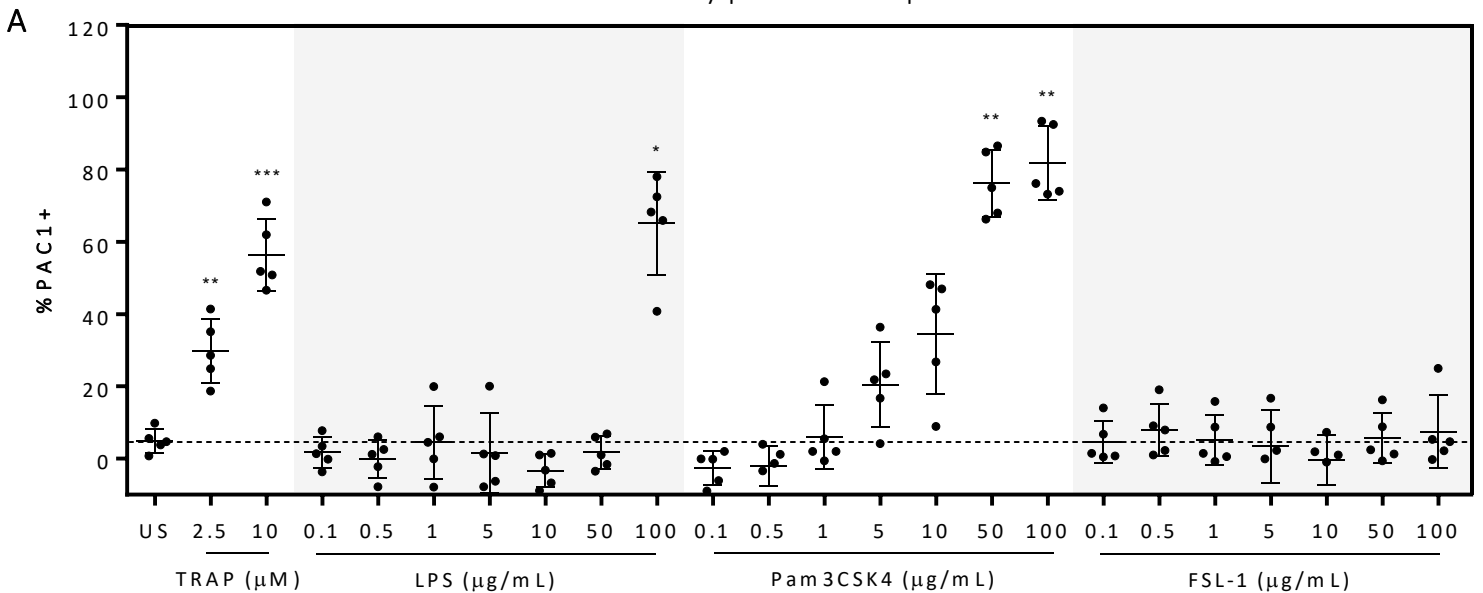

AMI platelet-rich plasma

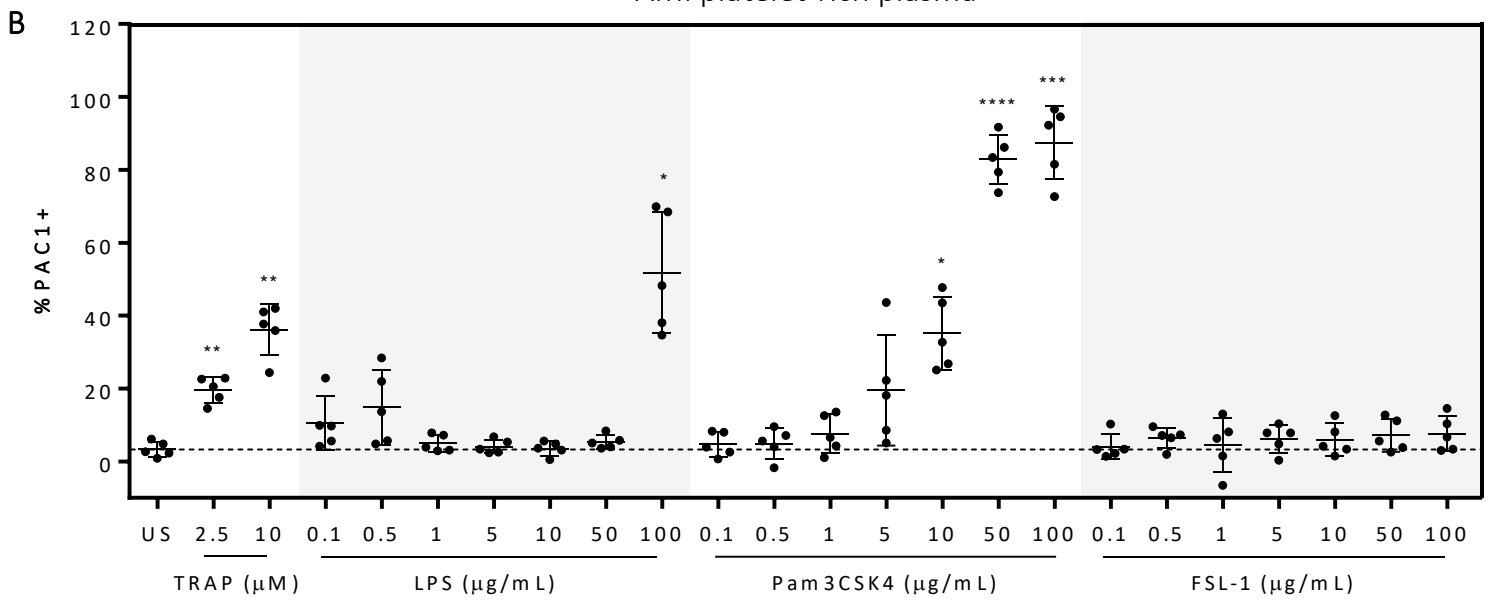

Figure 3.10 PAC1 is upregulated on platelets following incubation with TLR agonists in PRP.

PAC1 positivity was assessed in 5 healthy (A) and 5 AMI subjects (B) in PRP. Within each cohort, one-way ANOVA was found to be significant $(p<0.05)$ and Tukey's multiple comparisons test was performed to detect differences. $*$ indicates that the value for the treatment is significantly different than the unstimulated sample. Mean \pm SD for 5 subjects from each cohort is shown. ${ }^{*} \mathrm{p}<0.05, * * \mathrm{p}<0.01, * * * \mathrm{p}<0.001, * * * * \mathrm{p}<0.0001$. The dotted line marks the mean level of platelet activation in unstimulated platelets. 
Healthy whole blood

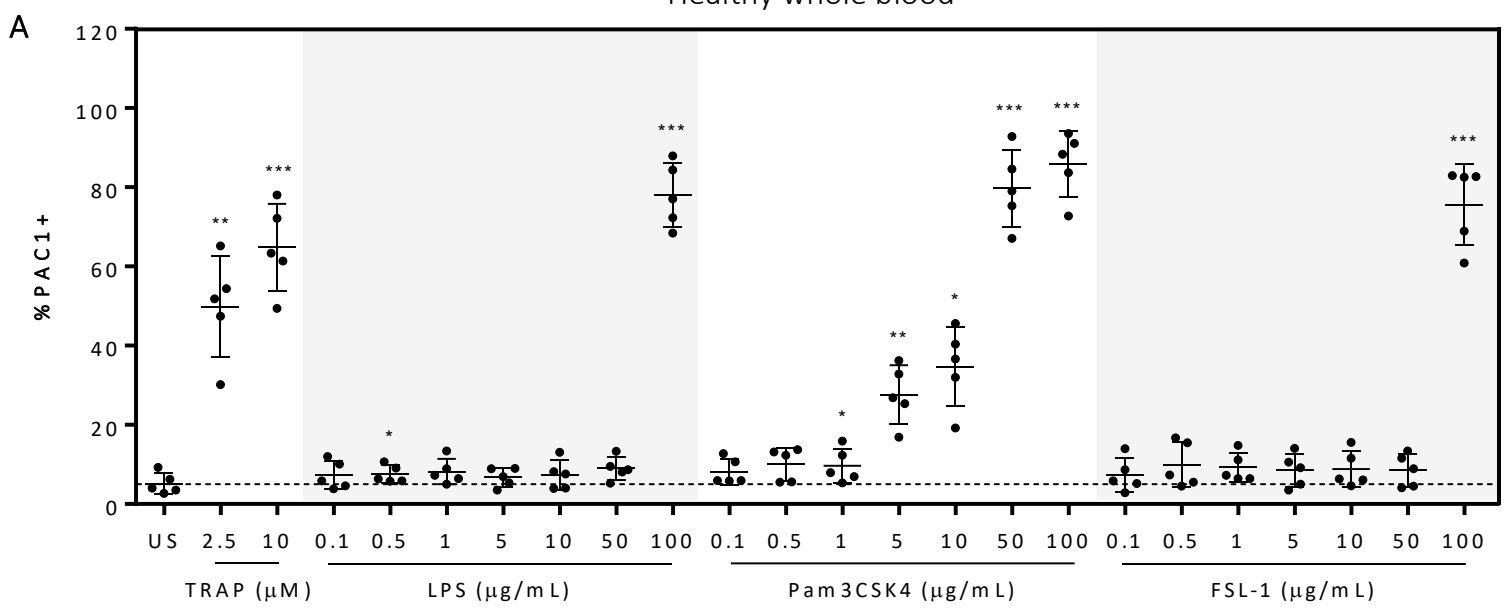

AMI whole blood

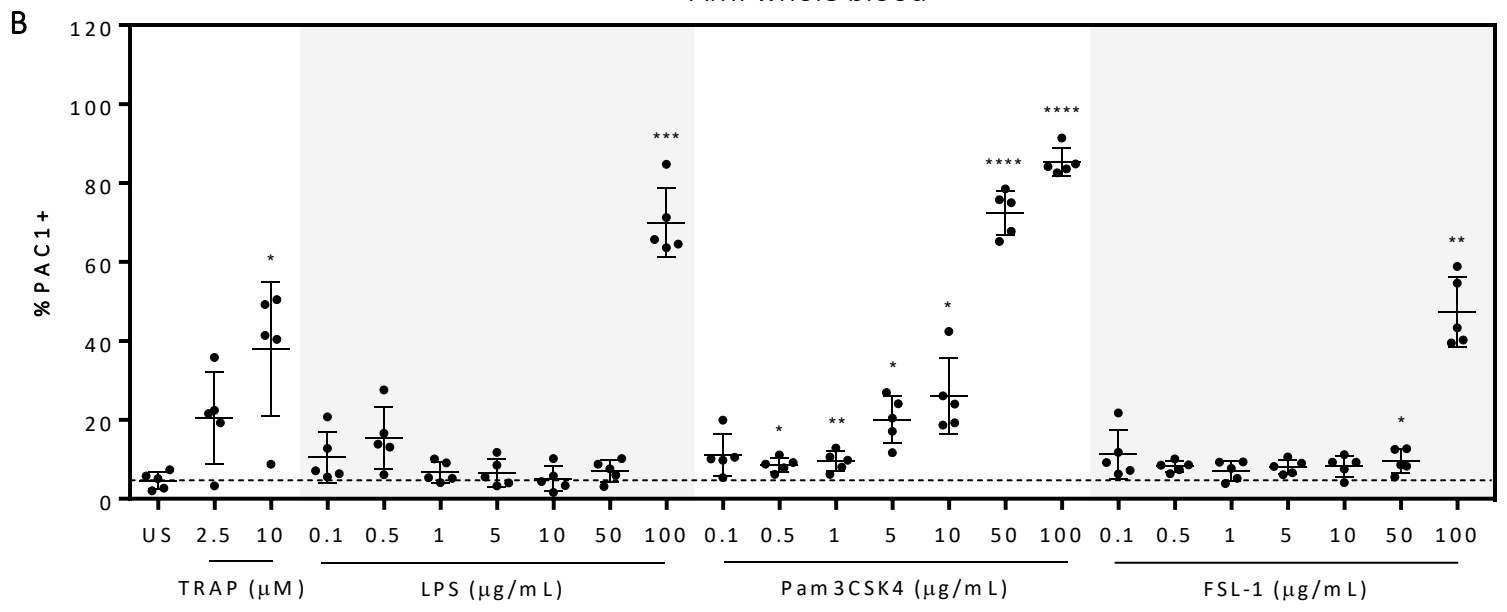

Figure 3.11 Variable PAC1 expression is seen in response to TLR agonism in WB.

PAC1 positivity was assessed in 5 healthy $(A)$ and 5 AMI subjects (B) in whole blood. Within each cohort, one-way ANOVA was found to be significant $(p<0.05)$ and Tukey's multiple comparisons test was performed to detect differences. * indicates that the value for the treatment is significantly different than the unstimulated sample. Mean \pm SD for 5 subjects from each cohort is shown. ${ }^{*} p<0.05, * * p<0.01, * * * p<0.001, * * * * p<0.0001$. The dotted line marks the mean level of platelet activation in unstimulated platelets. 
Table 3.6 Incubation with a range of TLR agonists induces platelet PAC1-positivity in the healthy and AMI cohorts.

\begin{tabular}{|c|c|c|c|c|c|}
\hline & & \multicolumn{2}{|c|}{ Healthy } & \multicolumn{2}{|c|}{ AMI } \\
\hline & & WB & PRP & WB & PRP \\
\hline US & - & $5.2 \pm 2.7$ & $4.9 \pm 3.3$ & $4.6 \pm 2.2$ & $3.3 \pm 2.1$ \\
\hline \multirow{2}{*}{ TRAP $(\mu \mathrm{M})$} & 2.5 & $49.8 \pm 12.8 * *$ & $29.7 \pm 8.8^{* *}$ & $20.5 \pm 11.6$ & $19.6 \pm 3.5^{* *}$ \\
\hline & 10 & $64.9 \pm 11.0 * * *$ & $56.5 \pm 9.9 * * *$ & $38.1 \pm 17.0 *$ & $36.2 \pm 7.1^{* *}$ \\
\hline \multirow{7}{*}{ LPS $(\mu \mathrm{g} / \mathrm{mL})$} & 0.1 & $7.3 \pm 3.6$ & $1.7 \pm 4.2$ & $10.5 \pm 6.4$ & $10.5 \pm 7.4$ \\
\hline & 0.5 & $7.5 \pm 2.2$ & $-0.1 \pm 5.2$ & $15.5 \pm 7.8$ & $14.9 \pm 10.3$ \\
\hline & 1 & $8.2 \pm 3.2$ & $4.5 \pm 10.2$ & $6.8 \pm 2.6$ & $5.0 \pm 2.4$ \\
\hline & 5 & $6.7 \pm 2.3$ & $1.6 \pm 11.1$ & $6.6 \pm 3.5$ & $4.1 \pm 1.9$ \\
\hline & 10 & $7.4 \pm 3.8$ & $-3.2 \pm 4.6$ & $5.1 \pm 3.2$ & $3.5 \pm 2.0$ \\
\hline & 50 & $9.0 \pm 2.9$ & $1.7 \pm 4.5$ & $7.1 \pm 2.7$ & $5.4 \pm 1.9$ \\
\hline & 100 & $78.0 \pm 8.1^{* * *}$ & $65.1 \pm 14.3^{*}$ & $70.0 \pm 8.8^{* * *}$ & $51.9 \pm 16.6^{*}$ \\
\hline \multirow{7}{*}{$\begin{array}{c}\text { Pam3CSK4 } \\
(\mu \mathrm{g} / \mathrm{mL})\end{array}$} & 0.1 & $8.2 \pm 3.3$ & $-2.7 \pm 4.7$ & $11.1 \pm 5.3$ & $4.7 \pm 3.4$ \\
\hline & 0.5 & $10.1 \pm 4.1$ & $-2.1 \pm 5.7$ & $8.6 \pm 1.8^{*}$ & $4.9 \pm 4.2$ \\
\hline & 1 & $9.7 \pm 4.3^{*}$ & $6.0 \pm 8.8$ & $9.5 \pm 2.5^{* *}$ & $7.6 \pm 5.4$ \\
\hline & 5 & $27.6 \pm 7.4^{* *}$ & $20.5 \pm 11.6$ & $20.0 \pm 6.0^{*}$ & $19.5 \pm 16.2$ \\
\hline & 10 & $34.8 \pm 10.0 *$ & $34.4 \pm 16.6$ & $26.1 \pm 9.6^{*}$ & $35.2 \pm 10.1^{*}$ \\
\hline & 50 & $79.8 \pm 9.7 * * *$ & $76.1 \pm 9.3^{* *}$ & $72.5 \pm 5.7 * * * *$ & $82.9 \pm 6.8 * * * *$ \\
\hline & 100 & $85.9 \pm 8.2^{* * *}$ & $81.8 \pm 10.2 * *$ & $85.3 \pm 3.5^{* * * *}$ & $87.5 \pm 10.1^{* * *}$ \\
\hline \multirow{7}{*}{$\begin{array}{c}\text { FSL-1 } \\
(\mu \mathrm{g} / \mathrm{mL})\end{array}$} & 0.1 & $7.3 \pm 4.3$ & $4.6 \pm 5.8$ & $11.3 \pm 6.3$ & $4.1 \pm 3.5$ \\
\hline & 0.5 & $9.9 \pm 5.8$ & $7.9 \pm 7.1$ & $8.2 \pm 1.4$ & $6.5 \pm 2.8$ \\
\hline & 1 & $9.2 \pm 3.7$ & $5.1 \pm 7.0$ & $7.1 \pm 2.5$ & $4.5 \pm 7.4$ \\
\hline & 5 & $8.5 \pm 4.3$ & $3.4 \pm 10.2$ & $8.1 \pm 1.8$ & $6.2 \pm 3.8$ \\
\hline & 10 & $8.8 \pm 4.6$ & $-0.5 \pm 6.9$ & $8.3 \pm 2.7$ & $6.0 \pm 4.4$ \\
\hline & 50 & $8.5 \pm 4.1$ & $5.6 \pm 6.9$ & $9.5 \pm 3.1$ & $7.2 \pm 4.5$ \\
\hline & 100 & $75.6 \pm 10.2^{* * *}$ & $7.4 \pm 10.1$ & $47.3 \pm 8.9 * *$ & $7.6 \pm 4.9$ \\
\hline
\end{tabular}

Mean \pm SD for 5 subjects for each cohort is shown. Within each cohort, one-way ANOVA was found to be significant $(p<0.05)$ for each parameter and Tukey's multiple comparisons tests were performed. * indicates the value for the treatment is significantly different than the unstimulated sample. ${ }^{*} p<0.05, * * p<0.01, * * * p<0.001, * * * * p<0.0001$. 


\subsubsection{Platelet activation in response to Pam3CSK4.}

Across cohorts, incubation with Pam3CSK4 produced a dose-dependent increase in expression of both platelet activation markers. In PRP, CD62p expression was high following incubation with 100 $\mu \mathrm{g} / \mathrm{mL}$ Pam3CSK4 (Healthy, 84.7.0 \pm 7.8\%; AMI, $88.0 \pm 8.2 \%$; both $\mathrm{p}<0.0001$ ) and $50 \mu \mathrm{g} / \mathrm{mL}$ Pam3CSK4 (Healthy, 75.7 $\pm 8.7 \%$; AMI, $79.7 \pm 9.5 \%$; both $p<0.0001$ ) compared to the unstimulated sample (Figure 3.8). In WB, the CD62p expression level for each dose of Pam3CSK4 was comparable to that seen in PRP and were greater than expression in unstimulated platelets (all p<0.001, Figure 3.9). In both healthy and AMI platelets, CD62p expression in PRP following both 5 $\mu \mathrm{g} / \mathrm{mL}$ and $10 \mu \mathrm{g} / \mathrm{mL}$ Pam3CSK4 was numerically higher than expression in unstimulated platelets. For stimulation with $10 \mu \mathrm{g} / \mathrm{mL}$ Pam3CSK4 in the AMI cohort, this increase was statistically significant $(21.4 \pm 4.8 \%$ CD62p+, $p<0.01)$. In both healthy and AMI WB, CD62p expression for these doses was significantly greater than in the unstimulated sample (all $p<0.05$ ). At both of these concentrations, the response in WB was larger than in PRP for the healthy cohort (both $\mathrm{p}<0.05)$. This difference was also observed for incubation with $5 \mu \mathrm{g} / \mathrm{mL}$ Pam3CSK4 in the AMI cohort $(p<0.01)$. In assessing dose-response curves for CD62p expression in response to Pam3CSK4, 50\% of maximal platelet-CD62p expression was observed at a concentration of $21 \mu \mathrm{g} / \mathrm{mL}$ and $20 \mu \mathrm{g} / \mathrm{mL}$ in healthy and AMI PRP, respectively (Figure 3.12). Similar concentrations of Pam3CSK4 achieved $50 \%$ of maximal platelet-CD62p expression in WB (Figure 3.12). Similar quantitative increases in PAC1 expression were observed for Pam3CSK4 stimulation (Figure 3.10 and Figure 3.12). 

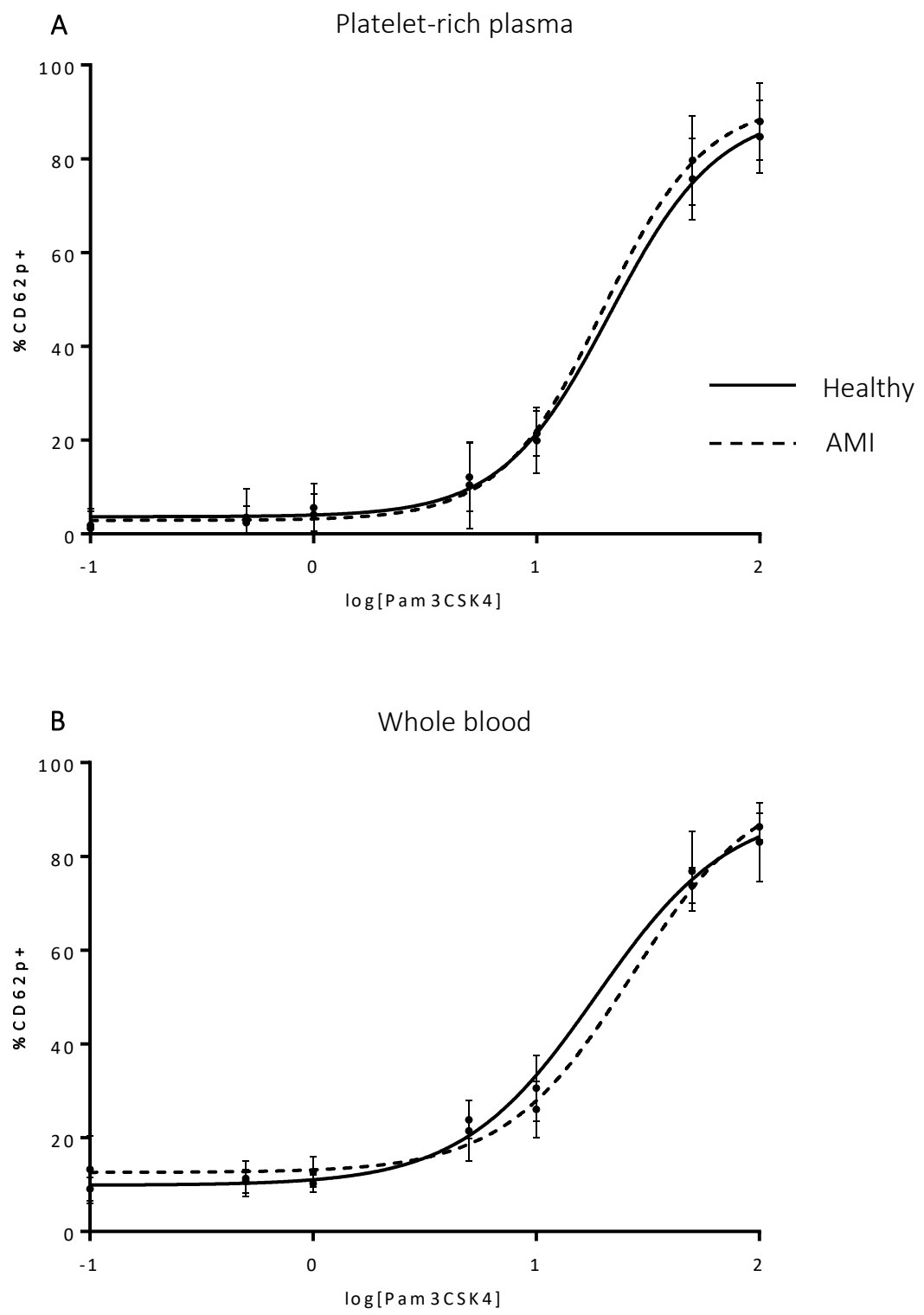

Figure 3.12 Platelets are dose-dependently activated in response to Pam3CSK4.

The percentage of platelets that were CD62p-positive was measured following incubation with $0.1-100 \mu \mathrm{g} / \mathrm{mL}$ Pam3CSK4 in PRP (A) and WB (B). The solid black curve represents the healthy cohort and the dotted black curve represents the AMI cohort. Agonist concentrations were log transformed and a sigmoidal curve was fitted. Mean \pm SD for 5 subjects in each cohort is shown. 


\subsubsection{Platelet activation in response to FSL-1 stimulation.}

In PRP, platelets did not express CD62p following incubation with FSL-1, a TLR2/6 agonist, at any dose tested in this study (Figure 3.8). However, in comparison to unstimulated platelets in WB, a significant increase in CD62p was observed following stimulation with $100 \mu \mathrm{g} / \mathrm{mL}$ FSL-1 (Healthy, $77.4 \pm 9.2 \%$ CD62p+, $p<0.001 ; A M I, 53.9 \pm 7.2 \%, p<0.01 ;$ Figure 3.9). Platelet-CD62p expression in response to this stimulation was greater in healthy WB than in AMI WB ( $p<0.0001)$. High-dose FSL1 incubation produced significant platelet-PAC1 expression in WB across cohorts (both $p<0.01$; Figure 3.11) but no increase in PAC1-positivity was seen in PRP at any dose of FSL-1 tested (Figure 3.10).

\subsubsection{Platelet-monocyte aggregation (PMAgg) in response to TLR agonists.}

PMAgg was assessed by determining changes in MFI of the platelet-specific marker, CD42a, gated on CD64+ monocytes. PMAgg determines the involvement of platelets in initiating or potentiating immune responses to these TLR agonists and is also a marker of platelet activation. PMAgg was not statistically significantly different following incubation with any dose of LPS for either the healthy (Figure 3.13A) or AMI (Figure 3.13B) cohort. In contrast, PMAgg in response to Pam3CSK4 increased in a dose-dependent manner in both healthy and AMI subjects. Significant increases in PMAgg were recorded for 100 mg/mL (Healthy, MFI $201.6 \pm$ 42.8; AMI, $537 \pm 127.2$; both p<0.01) and $50 \mu \mathrm{g} / \mathrm{mL}$ Pam3CSK4 (healthy, $131.4 \pm 22.2$; AMI, $420.2 \pm 36.9$; both $\mathrm{p}<0.05$ ) compared to unstimulated platelets. At both of these concentrations, PMAgg was significantly greater in the AMI group (both $\mathrm{p}<0.0001$ ). For the AMI group, incubation with $10 \mu \mathrm{g} / \mathrm{mL}$ Pam3CSK4 also produced significant PMAgg (MFI $121.0 \pm 36.9$, $\mathrm{p}<0.01$ ). At the highest dose of FSL-1 used (100 $\mu \mathrm{g} / \mathrm{mL})$, PMAgg was significantly increased in the healthy cohort $(159.5 \pm 44.5, \mathrm{p}<0.05)$ and was slightly, but not significantly, elevated in the AMI cohort $(61.4 \pm 12.9)$ in comparison to unstimulated platelets. This response to high-dose FSL-1 was significantly different across cohorts $(p<0.01)$. All results for PMAgg are presented in Table 3.7. 
Healthy platelet-monocyte aggregation

A

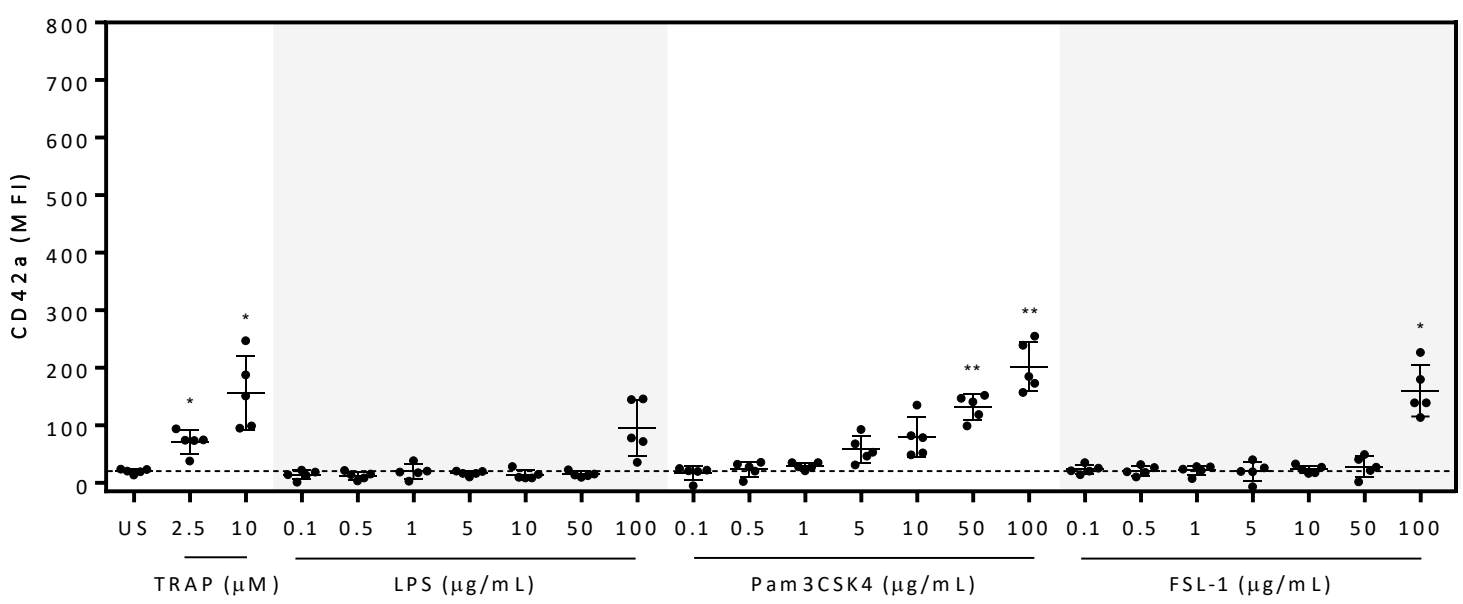

AMI platelet-monocyte aggregation

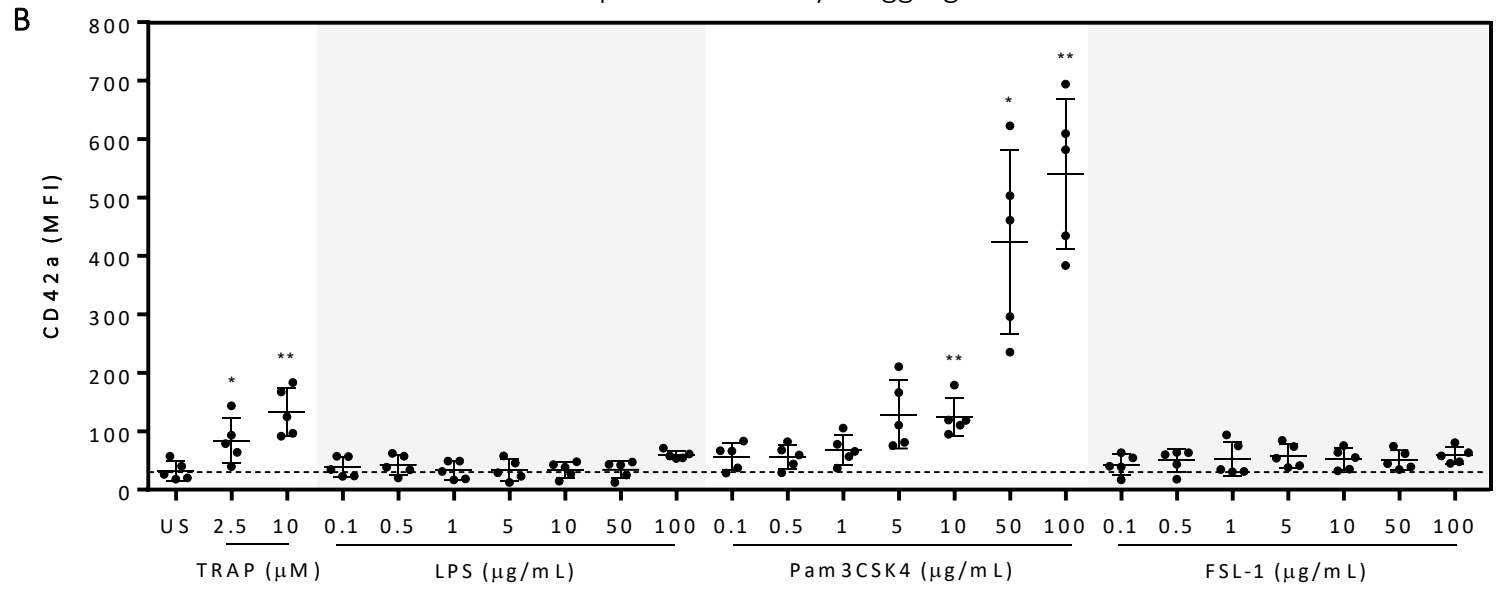

Figure 3.13 Increased PMAgg occurs following TLR agonism in the healthy and AMI cohort.

PMAgg was assessed in healthy (A) and 5 AMI (B) subjects. Within each cohort, one-way ANOVA was found to be significant $(p<0.05)$ for each parameter and Tukey's multiple comparisons test was performed to detect differences. * indicates that the value for the treatment is significantly different than the unstimulated sample. Mean \pm SD for 5 subjects in each cohort is shown. ${ }^{*} p<0.05,{ }^{* *} p<0.01$. The dotted line marks the mean level of PMAgg in unstimulated platelets. 
Table 3.7 Increased PMAgg occurs following incubation with a range of TLR agonists in the healthy and AMI cohorts.

\begin{tabular}{|c|c|c|c|}
\hline & & Healthy & AMI \\
\hline US & -- & $20.1 \pm 4.0$ & $32.3 \pm 16.2$ \\
\hline \multirow{2}{*}{ TRAP $(\mu \mathrm{M})$} & 2.5 & $70.7 \pm 20.4^{*}$ & $83.7 \pm 38.9$ \\
\hline & 10 & $155.8 \pm 63.9 *$ & $132.8 \pm 41.3^{* *}$ \\
\hline \multirow{7}{*}{ LPS $(\mu \mathrm{g} / \mathrm{mL})$} & 0.1 & $13.8 \pm 7.9$ & $38.9 \pm 17.1$ \\
\hline & 0.5 & $12.5 \pm 6.8$ & $42.4 \pm 17.3$ \\
\hline & 1 & $19.5 \pm 12.8$ & $33.0 \pm 15.6$ \\
\hline & 5 & $16.6 \pm 4.0$ & $33.6 \pm 18.1$ \\
\hline & 10 & $13.9 \pm 8.3$ & $34.2 \pm 13.3$ \\
\hline & 50 & $14.7 \pm 5.0$ & $34.2 \pm 14.7$ \\
\hline & 100 & $95.2 \pm 48.7$ & $59.6 \pm 7.1$ \\
\hline \multirow{7}{*}{$\begin{array}{c}\text { Pam3CSK4 } \\
(\mu \mathrm{g} / \mathrm{mL})\end{array}$} & 0.1 & $16.6 \pm 12.2$ & $52.8 \pm 27.4$ \\
\hline & 0.5 & $23.6 \pm 13.2$ & $52.8 \pm 24.6$ \\
\hline & 1 & $29.1 \pm 6.2$ & $64.8 \pm 31.6$ \\
\hline & 5 & $58.2 \pm 23.4$ & $125.2 \pm 62.3$ \\
\hline & 10 & $79.2 \pm 34.6$ & $121.0 \pm 36.9 * *$ \\
\hline & 50 & $131.4 \pm 22.2^{* *}$ & $420.2 \pm 156.4^{*}$ \\
\hline & 100 & $201.6 \pm 42.8^{* *}$ & $537.2 \pm 127.2 * *$ \\
\hline \multirow{7}{*}{$\begin{array}{c}\text { FSL-1 } \\
(\mu \mathrm{g} / \mathrm{mL})\end{array}$} & 0.1 & $23.1 \pm 7.7$ & $39.1 \pm 20.2$ \\
\hline & 0.5 & $21.0 \pm 8.4$ & $46.2 \pm 27.1$ \\
\hline & 1 & $21.6 \pm 8.4$ & $49.6 \pm 33.5$ \\
\hline & 5 & $19.8 \pm 17.0$ & $54.6 \pm 25.8$ \\
\hline & 10 & $23.4 \pm 6.7$ & $48.7 \pm 24.3$ \\
\hline & 50 & $28.0 \pm 18.0$ & $47.3 \pm 22.1$ \\
\hline & 100 & $159.5 \pm 44.5^{*}$ & $61.4 \pm 12.9$ \\
\hline
\end{tabular}

Mean \pm SD for 5 subjects in each cohort is shown. Within each cohort, one-way ANOVA was found to be significant $(p<0.05)$ for each parameter and Tukey's multiple comparisons tests were performed. ${ }^{*} p<0.05, * * p<0.01$. 


\subsubsection{Dose-dependent monocyte activation in response to TLR agonism.}

Monocyte activation, as assessed by cell-surface CD63 MFI, was measured in response to 0.01 to $100 \mathrm{ng} / \mathrm{mL}$ LPS and FSL-1 stimulation to ensure the efficacy of these agonists in healthy subjects. Incubation with $0.1-100 \mathrm{ng} / \mathrm{mL}$ LPS resulted in significantly increased expression of CD63 on monocytes (all p<0.05; Figure 3.14Error! Reference source not found.). In response to $100 \mathrm{ng} / \mathrm{mL}$

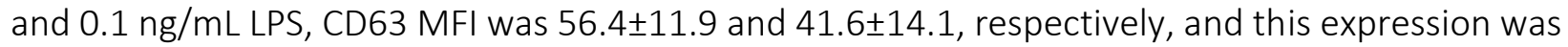

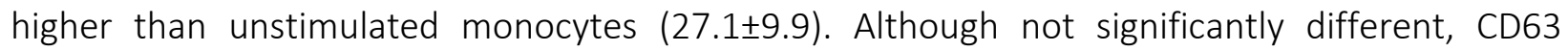

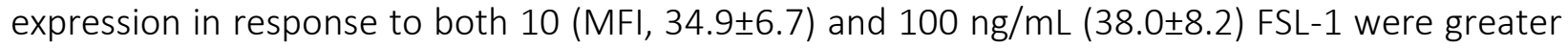
than expression recorded in the unstimulated monocyte population.

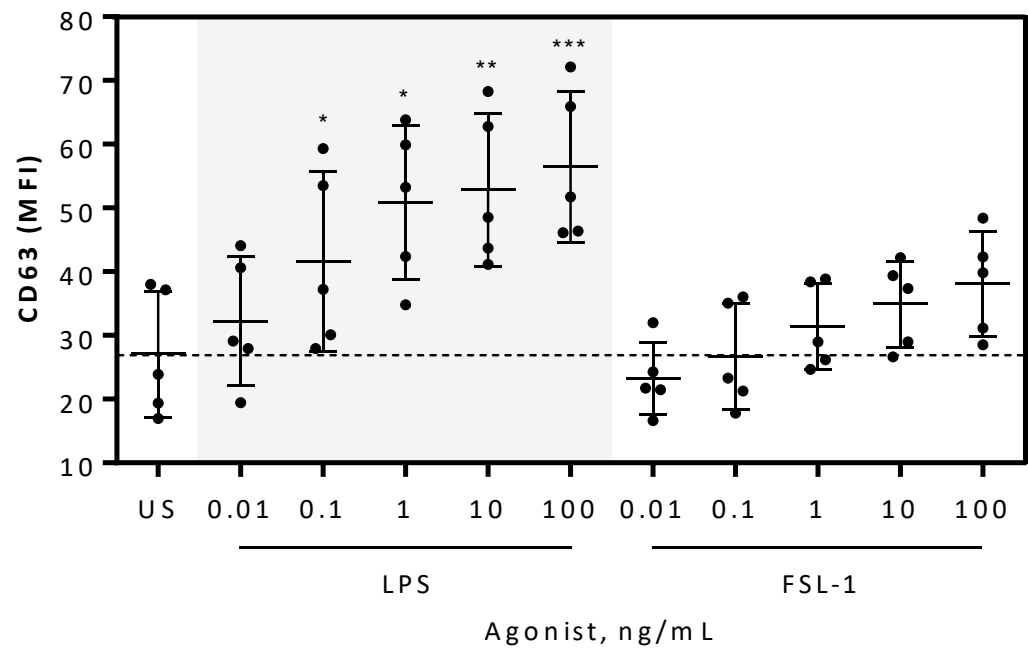

Figure 3.14 A dose-dependent increase in monocyte activation was observed in response to TLR agonism.

CD63 MFI in the unstimulated sample and each stimulated sample was measured following incubation with 0.01 $100 \mathrm{ng} / \mathrm{mL}$ of each TLR agonist. One-way ANOVA was found to be significant $(\mathrm{p}<0.05)$ for each parameter and Tukey's multiple comparisons test was performed to detect differences. ${ }^{*}$ indicates that the value for the treatment is significantly different than the unstimulated sample. Mean \pm SD for 5 healthy subjects is shown. ${ }^{*} p<0.05, * * p<0.01$, *** $p<0.001$. The dotted line marks the mean level of CD63 expression in the unstimulated sample. 


\subsection{Discussion.}

We have demonstrated that platelets differentially upregulate TLRs following AMI and exhibit distinct activation patterns in response to various TLR agonists in both healthy and AMI subjects. TLR1 and TLR4 were significantly upregulated in AMI platelets and platelet-TLR2 expression may be increased, but to a lesser extent, following AMI. In contrast, platelet-TLR6 expression was unchanged post-AMI. Platelets were directly activated in a dose-dependent manner in response to the TLR2/1 agonist, Pam3CSK4, and this response was similar in both healthy and AMI subjects. Platelets were directly activated in response to a high dose $(100 \mu \mathrm{g} / \mathrm{mL})$ of the TLR4 agonist, LPS, and this response was also similar across cohorts. There was no evidence of direct platelet activation in response to FSL-1, a TLR 2/6 agonist, although at a high dose we observed evidence of potent indirect activation in WB and this response was larger in healthy subjects. A dosedependent increase in PMAgg was seen in response to Pam3CSK4 in both cohorts and, for stimulation with high-dose Pam3CSK4, PMAgg was greater in AMI subjects. PMAgg was also significantly elevated following high-dose FSL-1 stimulation in healthy, but not AMI, subjects.

Expression of TLR1, 2, 4 and 6 on and within platelets has been reported previously [185, 187, 188], but how TLR expression changes in AMI remains relatively unexplored. We are the first to report that total protein expression of TLR1 and TLR4 is upregulated in platelets from AMI patients. We suggest that platelets are capable of selectively inducing TLR protein synthesis in response to AMI. TLR1 and TLR4 were upregulated by $50 \%$ in AMI platelets and platelet-TLR2 was upregulated by $20 \%$ in AMI patients, although this study was underpowered to detect the change in TLR2 expression as significant. We suggest that platelets are able to increase protein synthesis of a distinct sub-set of TLRs in response to AMI.

We (see Chapter 2) and others [187] have previously shown that platelet-TLR expression is elevated following platelet activation in response to pro-thrombotic agonists in healthy subjects. Extensive platelet activation occurs during $\mathrm{AMI}$ and this activation is a likely cause for acute 
platelet-TLR upregulation. Gurses et al. have recently reported a step-wise increase in plateletsurface expression of TLR2 and TLR4 in patients with stable angina and patients with AMI in comparison to healthy controls [275]. These results indicate that, alongside increased protein synthesis of some TLRs, platelets are also able to upregulate cell-surface expression of these receptors during AMI. Furthermore, platelets may also upregulate TLR expression in a chronic fashion during atherosclerotic development, although to a lesser extent than the upregulation seen in AMI. Similar modulation of monocyte-TLR expression has been reported during atherosclerosis $[276,277]$, and suggests that global immune cell-TLR expression may be linked with developing an increasingly inflammatory plaque environment.

We also described a bimodal expression of platelet-TLR1, 4 and 6 in AMI subjects, with some AMI subjects exhibiting high platelet-TLR-expression. It is possible that AMI subjects can be differentially classified according to whether their platelet-TLR1, 4, and 6 expression is 'responsive' or 'non-responsive' to AMI. If this is the case, we may be able to identify a group of subjects whose platelets are hypersensitive to the DAMP-enriched environment during AMI and who may be, as a result, at an increased risk of persistent platelet activation and thrombosis post-AMI. This is an interesting finding of this study, and warrants further investigation in a larger cohort of healthy and AMI subjects.

Elevated expression of particular TLRs may heighten platelet sensitivity towards specific TLR agonists and this may predispose platelets to a pro-thrombotic phenotype during and following an AMI. Agonizing TLRs in healthy platelet-only suspensions induces a number of pro-inflammatory $[216,278,279]$ and pro-thrombotic $[198,280]$ platelet responses, although there is little information on how TLR-mediated platelet activation changes in AMI and following anti-platelet therapy. For this study, each TLR agonist was incubated in both PRP and WB. Incubation in PRP assessed platelet activation that was as a result of direct agonist interaction with the platelet-TLR. WB further assessed whether there was any amplification of platelet activation due to activation of indirect 'off-platelet' TLR pathways. CD62p and PAC1 are commonly used to assess platelet 
activation by two distinct pathways and are surrogate markers for degranulation and aggregation, respectively. CD62p is released from intracellular granules and expressed on the platelet surface following activation. Alternatively, PAC1 recognizes GP IIb/IIla, an activation-dependent integrin complex which binds to fibrinogen and, in this manner, facilitates platelet aggregation by bridging GPIIb/IIla complexes on adjacent platelets.

We found that platelets from both healthy and AMI subjects became dose-dependently activated in response to the prototypical TLR2/1 agonist, Pam3CSK4. High-dose Pam3CSK4 potently activated platelets similarly across WB and PRP, and incubation with lower concentrations produced moderate platelet activation which was greater in WB than in PRP. The platelet response to strong Pam3CSK4 stimulation is driven almost entirely by direct platelet activation, as seen by the results in PRP. The greater platelet response in WB at moderate doses is likely due to indirect platelet activation by stimulation of 'off-platelet' TLR2/1 pathways. Leukocyte activation can cause platelet activation [259, 260] and may play a role in potentiating TLR2/1-mediated platelet activation in WB at a particular dose range. We are the first to demonstrate that Pam3CSK4 is able to dose-dependently activate platelets from AMI patients despite treatment with DAPT. Pam3CSK4-mediated platelet-leukocyte aggregation in healthy subjects has been demonstrated previously [281], and we are the first to demonstrate that PMAgg in response to Pam3CSK4 is significantly higher in AMI subjects than in healthy subjects, indicating that Pam3CSK4-mediated PMAgg is heightened in AMI and not inhibited by DAPT. Combined with our observation that expression of TLR1 and possibly TLR2 was increased in the AMI cohort, these results suggest that the platelet-TLR2/1 complex may represent an alternative platelet activation pathway in AMI that is not adequately inhibited by current anti-platelet therapy. AMI platelets remain responsive to Pam3CSK4, and can elicit both potent pro-thrombotic responses (activation) and inflammatory responses (PMAgg) as a result.

In contrast to Pam3CSK4 stimulation, platelets exhibited very different activation patterns in response to LPS, a prototypical TLR4 agonist, and FSL-1, a prototypical TLR2/6 agonist. Across both 
PRP and WB, high-dose LPS potently activated platelets from both cohorts but did not induce platelet activation at any other dose tested. LPS is able to potently activate other immune cells, but there are conflicting reports regarding LPS-mediated platelet activation. Some [197, 198] describe increased CD62p expression following incubation with $\leq 10 \mu \mathrm{g} / \mathrm{mL}$ LPS but platelet activation under similar conditions was not described by others $[200,278,280]$. Similarly, LPSmediated platelet-leukocyte aggregation is reported inconsistently $[278,280]$, and LPS may also negatively regulate platelet aggregation to some extent [202]. In this study, platelets were unresponsive to LPS within a stimulation time frame that is sufficient to achieve potent platelet activation using Pam3CSK4. Therefore, it is unclear how increased platelet-TLR4 expression may be physiologically relevant in AMI. It may be that platelets elicit other, perhaps more immunedriven, responses to LPS that were not examined in this study. We have shown that the LPS used in this study was functional as LPS was able to dose-dependently activate monocytes. Taken together, these results demonstrates that LPS selectively acts on most, but not all, cell types in the blood.

Similarly, platelets did not become directly activated in response to FSL-1 at any dose tested in this study. Significant platelet activation was observed in WB only in response to high-dose FSL-1. This platelet response is largely attributed to activation of other TLR2/6 pathways in blood that are able to indirectly stimulate platelets. This was not due to the use of a non-functional agonist, as we showed that FSL-1 dose-dependently activated monocytes. The level of platelet activation in response to FSL-1 in WB was higher in healthy subjects. This may reflect a level of platelet inhibition by DAPT; platelets are only indirectly activated by FSL-1 and DAPT-treated platelets may be less responsive to activation signals that are initiated by other indirect TLR2/6 pathways in the blood.

Platelet-monocyte aggregation (PMAgg) was also used to assess the platelet response to TLR agonism. PMAgg is an important pro-inflammatory platelet response [282], can be predictive of thrombosis [283], and synergizes the individual cellular responses. These heterotypic aggregates 
are elevated following $\mathrm{AMI}[74,284]$ and we see a slight elevation in baseline PMAgg in AMI subjects in this study. We also show an increase in PMAgg only in response to high-dose LPS (only statistically significant in healthy subjects) and FSL-1 incubation and there is a dose-dependent increase in PMAgg in response to Pam3CSK4.

The PMAgg response to FSL-1 is similar to the platelet activation response to FSL-1 in WB. We suggest that, for FSL-1, the significant platelet activation observed in WB may be as a result of the formation of these heterotypic aggregates. As with FSL-1-mediated platelet activation, FSL-1mediated PMAgg was significantly higher in healthy subjects, and may be due to the absence of treatment with DAPT in this cohort compared to the AMI cohort. Of all the stimulation conditions, PMAgg was most strongly induced in the AMI cohort in response to high-dose Pam3CSK4. These results suggest that DAPT does not dampen PMAgg, rather AMI platelets are more readily able to associate with monocytes when compared to healthy platelets, and this occurs despite potent anti-platelet therapy in AMI subjects.

We demonstrated that the PMAgg response to TLR2/1 stimulation was larger in AMI subjects. The level of PMAgg has been shown to be a useful early marker of AMI [77], and the difference in aggregation between the healthy and AMI cohorts may indicate an in vivo mechanism for PMAgg in AMI. Further to the results in this study, it is interesting to determine whether PMAgg is driven mainly by platelets or by monocytes and to determine the time-course of PMAgg in response to TLR2/1 stimulation during AMI.

Platelet activation and aggregation are important drivers of coronary thrombosis during AMI, and persistent platelet activation post-AMI can promote recurrent thrombosis despite DAPT. Alongside this, TLRs play an important role in initiating and propagating sterile inflammation in response to $\mathrm{AMI}$, and TLR activation has been shown to be detrimentally involved in myocardial I/R injury post-AMI $[183,285]$. This involvement in I/R injury is likely to be mediated by the release 
of endogenous TLR ligands, often extracellular matrix or intracellular-resident components and which include components such as high mobility group box 1 (HMGB1) [286] and hyaluronan [287]. We suggest that platelets may be activated via the TLR2/1 pathway during and following $\mathrm{AMI}$ and this pathway is not sufficiently therapeutically targeted by currently available anti-platelet agents. Platelet-TLR1 and, to a lesser extent, platelet-TLR2, is upregulated in AMI patients and the platelet-TLR2/1 complex is functional and able to dose-dependently induce direct platelet activation. Furthermore, TLR2/1-mediated platelet activation and PMAgg occurred in AMI subjects despite treatment with DAPT, indicating that this alternative platelet activation pathway is inadequately inhibited by current therapy. We also suggest that the platelet-TLR4 pathway is less likely, and the platelet-TLR2/6 pathway unlikely, to contribute to platelet activation during and post-AMI. 


\subsection{Limitations.}

We were not able to compare platelet-TLR expression and TLR-mediated platelet activation in AMI subjects before and after DAPT treatment, as patients with suspected AMI are given anti-platelet therapy either prior to or immediately upon arrival to hospital. Additionally, it is not clear what would constitute a physiologically relevant concentration of these TLR agonists that would occur during infection or sterile inflammation, but it is possible that high localized doses of these agonists exist post-AMI. We cannot exclude off-target effects of the TLR agonists used in this study, although these agonists have been used at similar doses in previous platelet activation studies $[198,281]$. Expansion of the size of the cohorts used for platelet-TLR expression $(n=12)$ and TLRmediated platelet activation $(n=5)$ may have allowed us to detect some changes in expression and activation as significant. We initially attempted to examine platelet-TLR expression by flow cytometry, but we were unable to produce a reproducible and consistent methodology for examining expression using this technique. We, therefore, used western blotting to examine platelet-TLR expression. However, we believe the size of these cohorts is appropriate, as this study was designed to capture large, and therefore potentially clinically significant, changes in expression or activation. Although all AMI patients were treated with DAPT prior to blood collection, it is possible that the level of platelet inhibition in response to DAPT varied across the AMI cohort. However, we are interested in determining platelet-TLR expression and activation in a real-world AMI cohort and each blood sample was collected at a sufficient time point after administration of DAPT to ensure that these drugs were biologically active. TLR agonism is likely to trigger a host of platelet functions that were not assessed in this study. In particular, platelets are known to induce downstream cellular immunomodulatory functions via interactions with leukocytes and endothelial cells as well as mediate responses to infection $[288,289]$, and these responses may exacerbate vascular inflammation post-AMI. However, platelet activation in response to TLR agonism was assessed in this study, as the activated platelet phenotype plays an important pro-thrombotic role in AMI. 


\subsection{Conclusion.}

In conclusion, we have demonstrated that TLR1 and TLR4 were significantly upregulated in AMI platelets and that TLR2 expression may also be increased, but to a lesser extent, following AMI. In addition, we have demonstrated dose-dependent platelet activation in response to the TLR2/1 agonist, Pam3CSK4, in healthy subjects and AMI subjects who have been treated with DAPT. Direct platelet activation also occurred in response to a high dose of the TLR4 agonist, LPS, but not in response to the TLR 2/6 agonist, FSL-1. It is possible that the platelet-TLR2/1 pathway is an alternative platelet activation pathway that may contribute to persistent platelet activation postAMI and despite treatment with DAPT. 
4 Chapter 4. The effects of aspirin and ticagrelor on TLR-mediated platelet activation: results of a randomised, cross-over trial. 


\subsection{Introduction.}

In our previous studies (Chapters 2 and 3), we demonstrated that stimulation of a range of platelet-TLRs in AMI subjects could lead to platelet activation despite pre-treatment with DAPT (a combination of aspirin with a $\mathrm{P}_{2} \mathrm{Y}_{12}$ receptor antagonist). In particular, we showed that platelets become directly and dose-dependently activated in response to a TLR2/1 and TLR9 agonist and become directly activated in response to high-dose TLR4 stimulation, but do not become directly activated in response to TLR2/6 agonism.

Interestingly, these patterns of platelet activation in AMI subjects were similarly observed in healthy subjects despite treatment of DAPT in the AMI cohort. We see two possibilities for the similarity in platelet activation patterns across these two cohorts: a) DAPT does not inhibit or only very modestly inhibits TLR-mediated platelet activation, or b) TLR-mediated platelet activation is greater in the absence of DAPT in AMI subjects, and DAPT inhibits this activation to a level seen in untreated healthy subjects. In our previous studies, we were unable to assess TLR-mediated platelet activation in the absence of anti-platelet therapy as patients with AMI receive this therapy prior to or immediately following presentation to hospital. Consequently, we could not establish the extent to which DAPT provides protection against TLR-mediated platelet activation. This problem was addressed in the current study.

We hypothesize that anti-platelet therapy does not inhibit, or only slightly inhibits platelet activation in response to direct TLR stimulation. Therefore, we aimed:

1. To determine the extent to which aspirin alone or in combination with ticagrelor, a potent $\mathrm{P}_{2} \mathrm{Y}_{12}$ receptor antagonist, can inhibit platelet activation in response to TLR2/1, TLR4 and TLR9 stimulation. 


\subsection{Materials and methods.}

\subsubsection{Study design.}

Ten healthy subjects (5 male, $49 \pm 6$ years) were recruited into a prospective single-blinded randomized cross-over trial. Exclusion criteria were: known cardiovascular or inflammatory disease, a platelet function or bleeding disorder, a platelet count of less than $100 \times 10^{9} / \mathrm{L}$, acute illness within 6 weeks prior to recruitment, pregnancy, diabetes mellitus and/or treatment with cardiovascular or immune-modulating drugs or treatment with anti-platelet agents within 7 days prior to recruitment This study was approved by the Health and Disability Ethics Committee, New Zealand (16/STH/240) and was registered with the Australian New Zealand Clinical Trials Registry (ACTRN12616001508460). All subjects provided written informed consent and the study was conducted in accordance with the Declaration of Helsinki. Subjects were randomized to receive either aspirin monotherapy (Cartia, Aspen Global Incorporated; 300 mg loading dose, 100 mg once daily maintenance dose) or DAPT, a combination of aspirin and ticagrelor (Brilinta, AstraZeneca; $180 \mathrm{mg}$ loading dose, $90 \mathrm{mg}$ bi-daily maintenance dose), for a total of 1 week. Following completion of the first anti-platelet drug regimen, subjects underwent a washout period of 3 weeks and were crossed over to the other anti-platelet drug regimen. Investigators, but not subjects, were blinded to the anti-platelet drug regimen that each subject was randomized to. The study cross-over protocol is presented in Figure 4.1.

Page / 129 


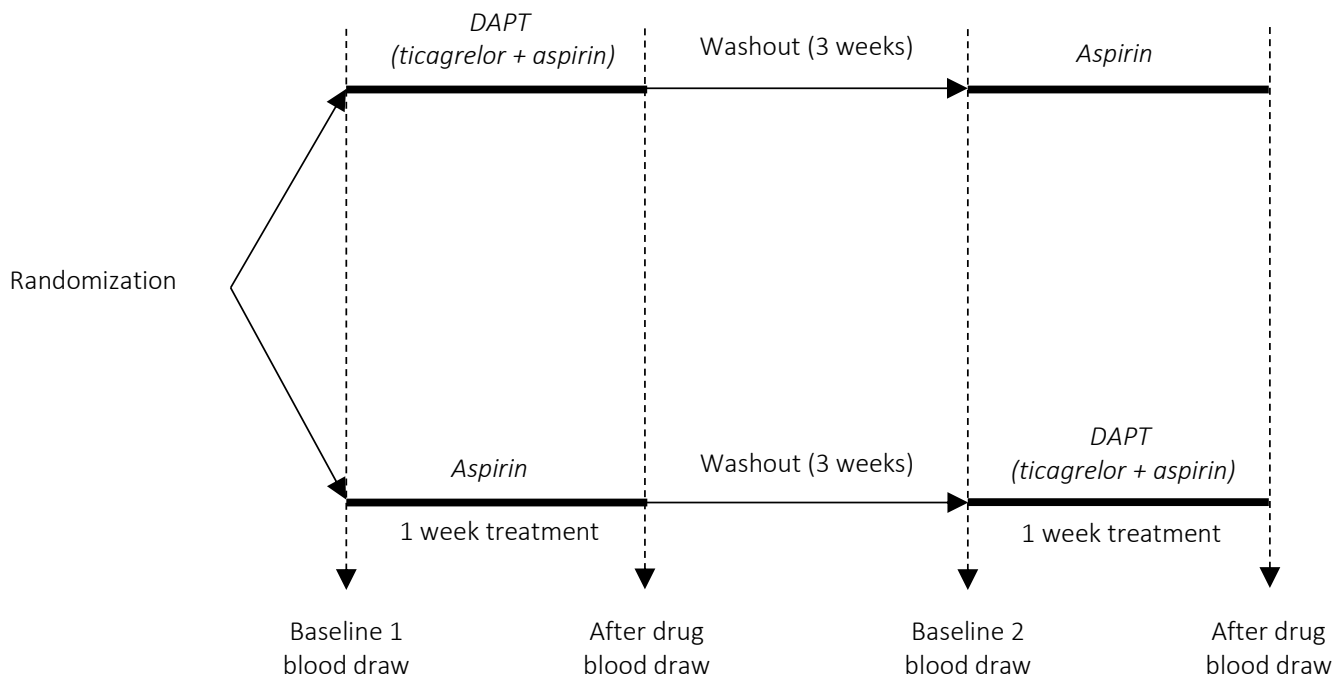

Figure 4.1 Study cross-over protocol.

10 healthy subjects were randomized to receive either aspirin monotherapy or DAPT, a combination of aspirin and ticagrelor, for 1 week. Following the first anti-platelet drug regimen, subjects had a washout period before commencing the second drug regimen for 1 week. Blood was drawn both before and after each drug regimen, for a total of 4 blood draws over the study period. 


\subsubsection{Blood drawing and platelet aggregation assay.}

Before and after each anti-platelet drug regimen, blood was collected from a peripheral vein into hirudin-anticoagulated tubes (Dynabyte). Platelet aggregation was assessed using a modification of light transmittance aggregometry using a Multiskan GO microplate spectrophotometer (Thermo Fisher Scientific) to confirm that each anti-platelet drug regimen was having the expected inhibitory effect on the platelet pathway that each drug targets. Blood was centrifuged at $200 \times \mathrm{g}$ for 12 minutes to produce PRP and at $1500 \times$ g for 12 minutes to produce platelet-poor plasma (PPP), and warmed to $37^{\circ} \mathrm{C}$. ADP $(6 \mu \mathrm{M})$ or AA $(500 \mu \mathrm{M})$ was added to PRP. ADP is an agonist for the $\mathrm{P}_{2} \mathrm{Y}_{12}$ receptor, which is inhibited by ticagrelor, and $\mathrm{AA}$ is an agonist for the COX-1 receptor, which is inhibited by aspirin. PBS was added, in an equal ratio, to warmed PPP. The absorbance was read at $620 \mathrm{~nm}$ at 0 minutes and at 8 minutes and all samples were shaken constantly between reads. Maximum platelet aggregation was calculated as follows (adapted from [290]):

Maximum aggregation $=100 \times \frac{\text { abs. PRP at } 0 \text { mins }- \text { abs. PRP at } 8 \text { mins }}{\text { abs. PRP at } 0 \text { mins }- \text { abs. PPP at } 8 \text { mins }}$

\subsubsection{Flow cytometric detection of TLR-mediated platelet activation.}

Before and after each anti-platelet drug regimen, flow cytometry was used to assess TLR-mediated platelet activation from each blood sample drawn. PRP was adjusted to $1 \times 10^{8}$ platelets $/ \mathrm{mL}$ with PBS and incubated for 15 minutes at room temperature with the following agonists (used in Chapters 2 and 3): TRAP6 (Tocris Bioscience), Pam3CSK4 (Tocris Bioscience), LPS (Enzo Life Sciences), and ODN2006 (InvivoGen). TRAP6, a protease-activated receptor agonist, is a universal platelet agonist that was used as a positive control for platelet activation. Platelets were incubated with 6 doses of TRAP6 $(0.2-7.5 \mu \mathrm{g} / \mathrm{mL})$, Pam3CSK4 (3.1-100.0 $\mu \mathrm{g} / \mathrm{mL})$ and ODN2006 (0.6-20.0 $\mu \mathrm{g} / \mathrm{mL})$ and a single high dose of LPS $(100 \mu \mathrm{g} / \mathrm{mL})$. A single dose of LPS was used as we have previously demonstrated that platelets only become activated in response to high-dose LPS (see Chapter 3). As a negative control, PRP was incubated with PBS. All samples of PRP were stained 
with anti-CD42a-PerCP (clone Beb-1), anti-CD62p-BV421 (clone AK4) and PAC1-FITC (clone PAC-1; Becton Dickinson) in staining buffer for 20 minutes in the dark at room temperature. The panel design for this study is show in Table 4.1. In parallel, PRP was incubated with PerCP-mouse IgG1,k, BV421-mouse IgG1, K or FITC-mouse IgM isotype controls. All antibodies and isotype controls were sourced from Becton Dickinson. All samples were fixed with 1\% paraformaldehyde and analysed on a FACSCanto II flow cytometer (Becton Dickinson). Flow cytometry data was analysed using FlowJo software (v10.0.7, Tree star). Platelets were identified by their side scatter properties and CD42a-positivity (Figure 4.2). The percentage of platelets that were positive for CD62p and PAC1 was determined (Figure 4.3). Flow cytometry analysis was performed by a study investigator (KH) who was blinded to the anti-platelet treatment of each subject.

Table 4.1 Panel design for assessing platelet activation in response to TLR agonism.

\begin{tabular}{|l|l|l|l|}
\hline & $\begin{array}{l}\text { Laser/channel } \\
\text { (fluorophore) }\end{array}$ & $\begin{array}{l}\text { Antibody target } \\
\text { (clone) }\end{array}$ & $\begin{array}{l}\text { Concentration in final staining } \\
\text { volume (final dilution) }\end{array}$ \\
\hline TLR-mediated & Blue/Red (PerCP) & CD42a (Beb1) & $0.25 \mu \mathrm{g} / \mathrm{mL}(1 \mathrm{in} \mathrm{50)}$ \\
platelet activation & $\begin{array}{l}\text { Blue/Green (FITC) } \\
\text { Violet/Blue (BV421) }\end{array}$ & PAC1 (PAC1) & $1.00 \mu \mathrm{g} / \mathrm{mL}(1 \mathrm{in} \mathrm{20)}$ \\
& CD62p (AK-4) & $1.00 \mu \mathrm{g} / \mathrm{mL}(1 \mathrm{in} \mathrm{100)}$ \\
\hline
\end{tabular}

A list of all antibody formats, clones, isotypes and manufacturers can be found in Appendix 1. 

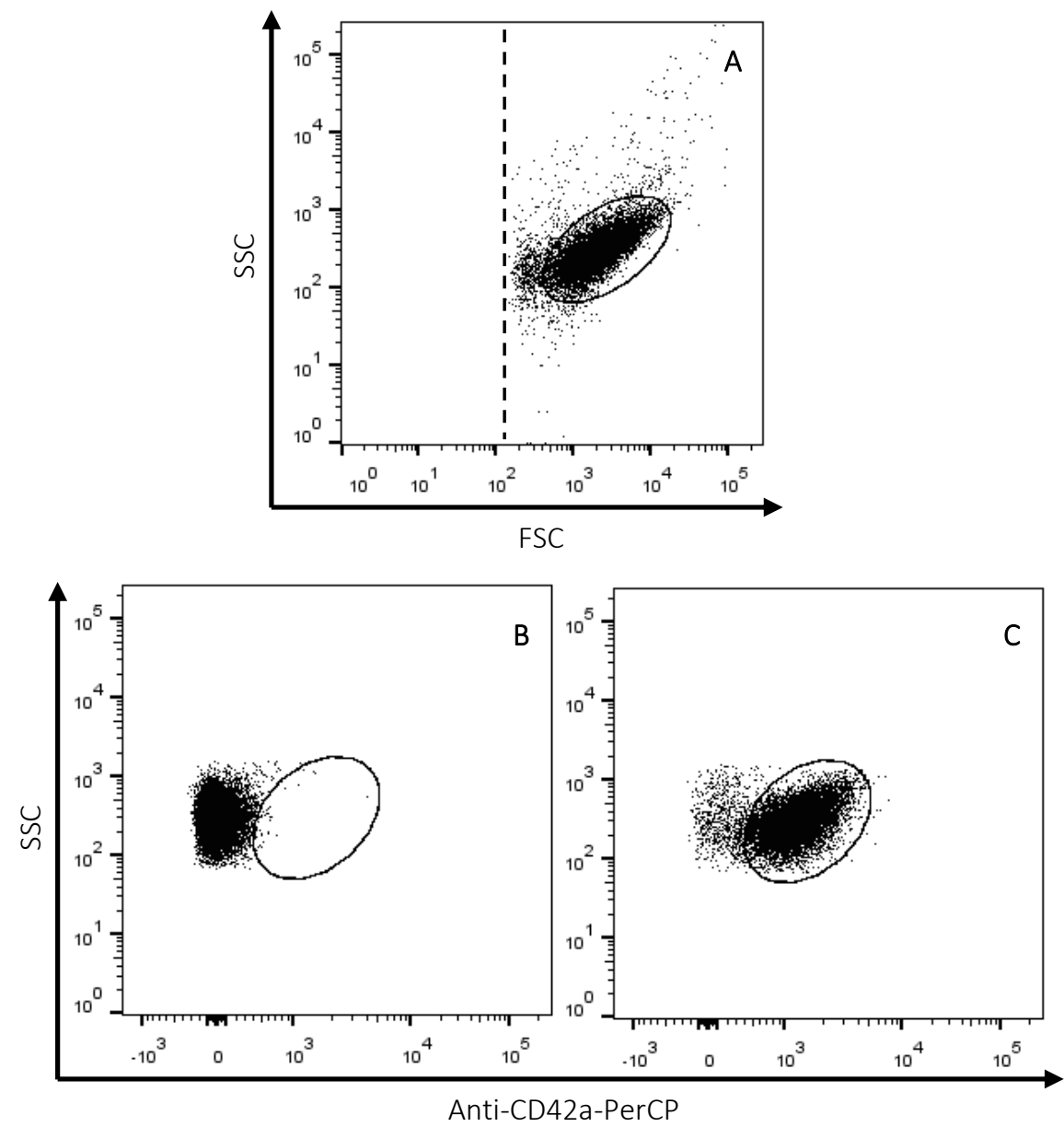

Figure 4.2 Platelets were identified by flow cytometry.

The platelet population was identified from PRP (A) by their forward and side scatter properties. Platelets were further identified by their CD42a-positivity (C) against the isotype control (B). 

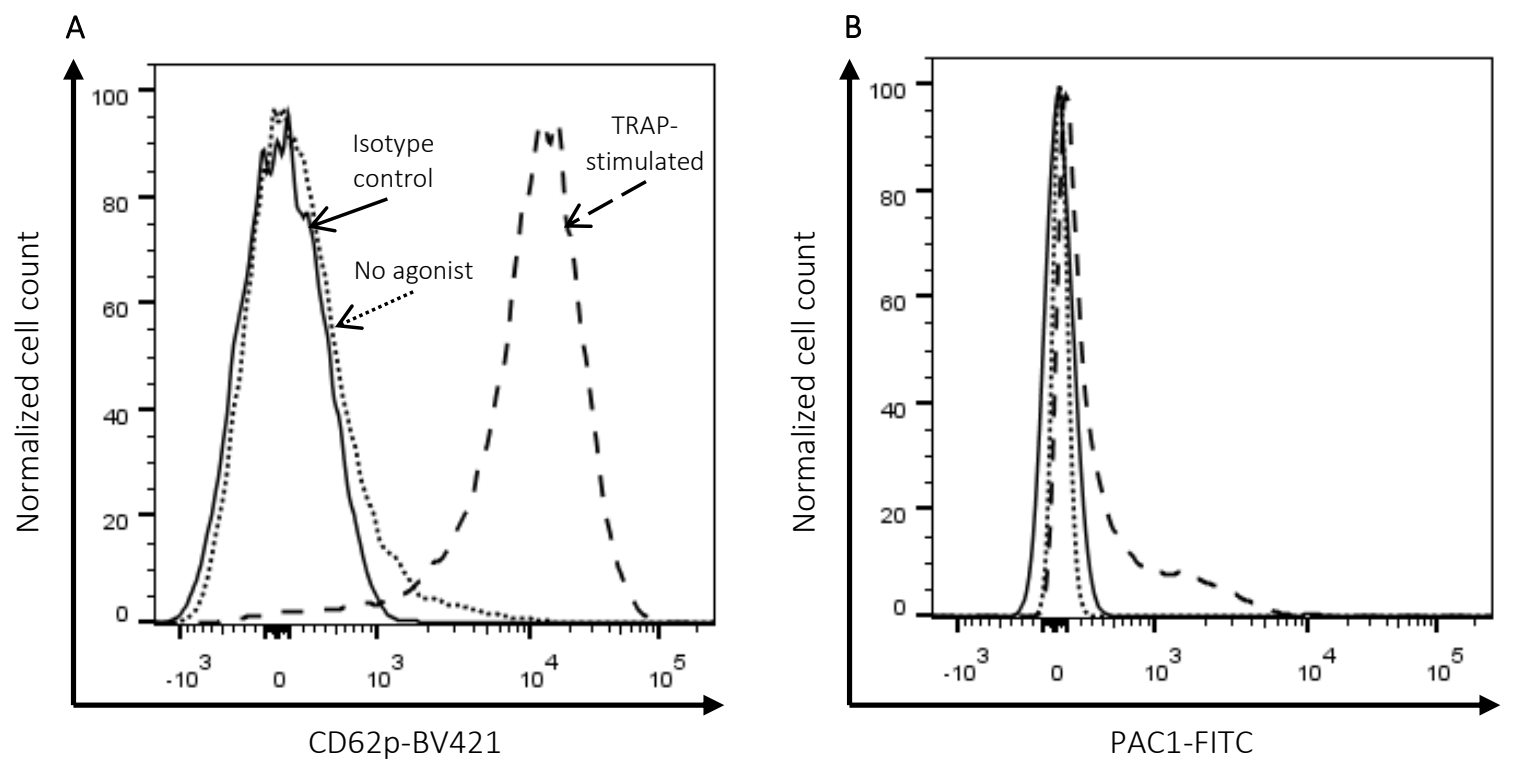

Figure 4.3 Representative plots of activation markers.

A representative plot of CD62p expression ( $A$ ) and PAC1 expression (B) is shown for unstimulated and TRAP-stimulated $(7.5 \mu \mathrm{g} / \mathrm{mL}) \mathrm{CD} 42 \mathrm{a}+$ platelets. Solid line, isotype control; dotted line, no agonist; dashed line, TRAP-stimulated. 


\subsubsection{Statistical analysis.}

With a sample size of 10 , we had the power to detect a $20 \%$ reduction in platelet activation in response to TLR stimulation following anti-platelet therapy with $90 \%$ power and $5 \%$ significance. This was based on the mean and standard deviation of platelet activation seen in response to TLR stimulation in healthy subjects in Chapters 2 and 3. Differences in maximum platelet aggregation measured before and after each anti-platelet drug regimen were examined using paired t-tests, and aggregation values were expressed as mean \pm SD. There was a potential period effect or potential treatment-by-period interaction due to the cross-over design for this study, and analysis for these effects was conducted (as described by Altman [291]) before analysing differences between baseline and drug-treatment measurements. For each parameter, the difference (đ; period 1 - period 2 ) and the average (ā; (period $1+$ period 2$) / 2$ ) for each individual was calculated and averaged in group A (individuals that received aspirin monotherapy (period 1) followed by aspirin and ticagrelor (period 2)) and group B (aspirin and ticagrelor (period 1) followed by aspirin monotherapy (period 2)). The period effect was tested by an unpaired t-test comparing differences (đ) (đ $\mathbb{d}_{1}$ vs. $-\left(đ_{2}\right)$ ) in the periods of group A and B. This confirmed whether or not one group benefited from receiving one drug regimen first, over the other. The treatment-by-period interaction was tested by an unpaired t-test comparing averages ( $\bar{a})\left(\bar{a}_{1} v s . \bar{a}_{2}\right)$ of the periods between group A and group B. This confirmed whether or not the wash-out period was sufficient, such that there was no carry-over of the effect of treatment. Differences in baseline measurements of platelet activation (baseline unstimulated vs. baseline stimulated samples) were examined using repeated measures one-way ANOVA with post-hoc Tukey's multiple comparisons tests. For TRAP, Pam3CSK4 and ODN2006, repeated measures two-way ANOVA was performed. If the interaction effect was found to be significant $(p<0.05)$, post-hoc Tukey's multiple comparisons tests were performed to detect differences. For LPS, repeated measures one-way ANOVA was performed and, if found to be significant $(p<0.05)$, post-hoc Tukey's multiple comparisons tests were performed to detect differences. All statistical analyses were performed using GraphPad Prism 7 (GraphPad Software Inc.). 


\subsection{Results.}

\subsubsection{Platelet aggregation.}

The platelet aggregation results from this study indicated effective aspirin- and DAPT-mediated platelet inhibition following each of these anti-platelet drug regimens. On aspirin monotherapy, maximal platelet aggregation in response to arachidonic acid was attenuated from $38 \pm 12 \%$ to $7 \pm 3 \%(p<0.0001)$ but aggregation was unaltered in response to ADP (Figure 4.4A). On DAPT, aggregation was attenuated from $52 \pm 16 \%$ to $5 \pm 2 \%$ in response to arachidonic acid and attenuated from $82 \pm 8 \%$ to $8 \pm 3 \%$ in response to ADP (both $p<0.001$, Figure $4.4 B$ ).
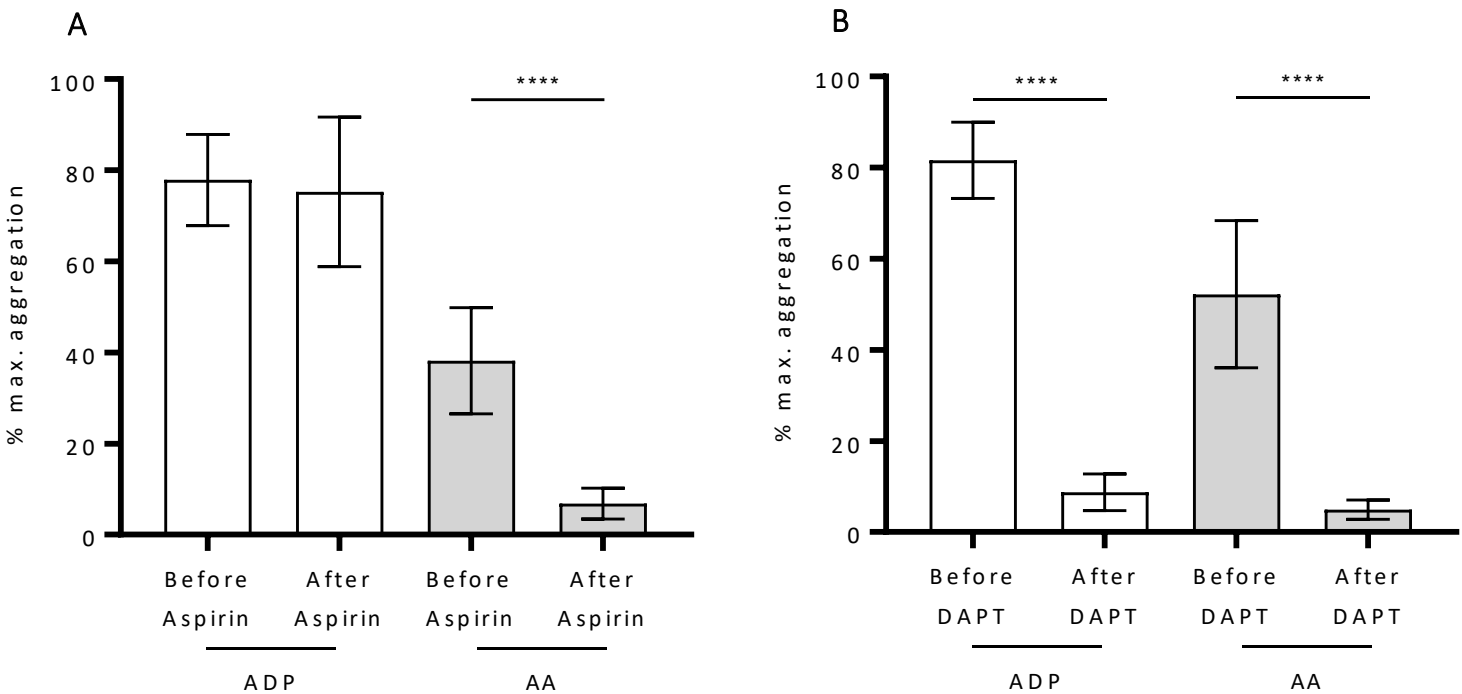

Figure 4.4 Platelet aggregation testing confirmed a significant inhibitory effect of the anti-platelet drug regimens on each of the targeted platelet activation pathways.

Aspirin monotherapy significantly attenuated platelet aggregation in response to arachidonic acid (AA) but not in response to ADP, indicating effective inhibition of only the AA pathway (A). DAPT significantly attenuated platelet activation in response to both $A A$ and ADP, indicating effective inhibition of both the AA and ADP pathways (B). Paired $\mathrm{t}$-tests were performed to detect differences. Mean $\pm \mathrm{SD}$ for 10 subjects is shown. ${ }^{* * * *} \mathrm{p}<0.0001$. 


\subsubsection{Period effect and treatment-by-period interaction analysis.}

For each parameter measured in this cross-over study, there was no statistically significant period effect or treatment-by-period interaction between group A, who received aspirin monotherapy followed by DAPT (aspirin and ticagrelor), and group B, who received DAPT followed by aspirin monotherapy. For example, the change in the percentage of platelets positive for PAC1 (\%PAC1positive platelets) in response to $7.5 \mu \mathrm{g} / \mathrm{mL}$ TRAP was calculated for each individual. This was defined as the percentage change between the baseline (before-drug) measurement and afterdrug measurement and was calculated for both group A (Table 4.2) and group B (Table 4.3). No difference between group $A$ and $B$ was noted for the differences in periods 1 and 2 ( $\mathbb{đ}_{1}$ and $-\left(đ_{2}\right)$ $(p=0.13))$, indicating no period effect. Also, there was no difference between group $A$ and $B$ for the average of periods 1 and $2\left(\bar{a}_{1}\right.$ and $\left.\bar{a}_{2}(p=0.44)\right)$, indicating no treatment-by-period interaction and demonstrating that the washout period was sufficient. This analysis was repeated for each parameter. We conclude that both baseline measurements were similar across groups $A$ and $B$, and each drug regimen was compared to the corresponding baseline without adjustment. 
Table 4.2 Group A received aspirin monotherapy (period 1) followed by DAPT (aspirin and ticagrelor) (period 2), $n=5$.

\begin{tabular}{|l|l|l|l|l|}
\hline \multirow{4}{*}{1} & $\begin{array}{l}\text { Period 1 } \\
\text { Aspirin monotherapy }\end{array}$ & $\begin{array}{l}\text { Period 2 } \\
\text { Aspirin and ticagrelor }\end{array}$ & $\begin{array}{l}\text { Period 1- } \\
\text { Period 2 }\end{array}$ & $\begin{array}{l}\text { (Period 1+ Period } \\
\text { 2)/2 }\end{array}$ \\
\cline { 2 - 5 } & -49.3 & -77.4 & 28.1 & -63.4 \\
\cline { 2 - 5 } & -48.7 & -83.6 & 34.9 & -66.2 \\
\cline { 2 - 5 } & 25.2 & -53.8 & 79 & -14.3 \\
\cline { 2 - 5 } & -15.4 & -73.7 & 58.3 & -44.6 \\
\cline { 2 - 5 } & -3.0 & -89.0 & $56.3\left(đ_{1}\right)$ & -46.0 \\
\hline Mean & -18.2 & -75.5 & 25.7 & 20.7 \\
\hline $\begin{array}{l}\text { Standard } \\
\text { deviation }\end{array}$ & 31.7 & 13.5 & & \\
\hline
\end{tabular}

$1 \%$ PAC1-positive platelets in response to $7.5 \mu \mathrm{g} / \mathrm{mL}$ TRAP expressed as \% change between before-drug and after-drug measurements.

Table 4.3 Group B received aspirin and ticagrelor (period 1) followed by aspirin monotherapy (period 2), $n=5$.

\begin{tabular}{|l|l|l|l|l|}
\hline \multirow{4}{*}{1} & $\begin{array}{l}\text { Period 1 } \\
\text { Ticagrelor and aspirin }\end{array}$ & $\begin{array}{l}\text { Period 2 } \\
\text { Aspirin monotherapy }\end{array}$ & $\begin{array}{l}\text { - (Period 1 - } \\
\text { Period 2) }\end{array}$ & $\begin{array}{l}\text { (Period 1 + Period } \\
\text { 2)/2 }\end{array}$ \\
\cline { 2 - 5 } & -65.5 & -31.3 & 34.2 & -48.4 \\
\cline { 2 - 5 } & -76.3 & -25.5 & 50.8 & -50.9 \\
\cline { 2 - 5 } & -90.17 & 1.8 & 92.0 & -44.19 \\
\cline { 2 - 5 } & -83.8 & 50.9 & 134.7 & -16.5 \\
\cline { 2 - 5 } & -81.3 & 17.1 & 98.4 & -32.1 \\
\hline Mean & -79.4 & 2.6 & 40.0 & $-38.4\left(\bar{a}_{2}\right)$ \\
\hline \multirow{2}{*}{$\begin{array}{l}\text { Standard } \\
\text { deviation }\end{array}$} & 9.2 & 33.5 & & 14.2 \\
\hline
\end{tabular}

$1 \%$ PAC1-positive platelets in response to $7.5 \mu \mathrm{g} / \mathrm{mL}$ TRAP expressed as \% change between before-drug and after-drug measurements. 


\subsubsection{TLR-mediated platelet activation at baseline.}

To investigate platelet activation in response to TLR agonism prior to anti-platelet drug administration, cell-surface expression of both PAC1 and CD62p was assessed by flow cytometry. During this cross-over study, two baseline measurements (Baseline 1 and Baseline 2) were taken (see Figure 4.1). These two baseline measurements were similar across all agonists tested here (Figure 4.5 and Figure 4.6). Dose-dependent increases in expression of both PAC1 and CD62p were observed following incubation with TRAP6, Pam3CSK4 and ODN2006. In comparison to unstimulated platelets, expression of both platelet activation markers significantly increased following incubation with the highest three doses of TRAP6 tested in this study (all $p<0.01$ ). Pam3CSK4-stimulated platelets exhibited increased PAC1-positivity and CD62p-positivity at all doses tested (all $p<0.05$ ). Similar to TRAP6, the three highest doses of ODN2006 tested here induced significant expression of activation markers in comparison to unstimulated platelets (all $\mathrm{p}<0.01)$. A single dose $(100 \mu \mathrm{g} / \mathrm{mL})$ of LPS was used in this study, as we have previously demonstrated that platelets only become activated in response to high-dose LPS (see Chapter 3). Following incubation with high-dose LPS, PAC1 expression and CD62p expression was significantly increased in comparison to unstimulated platelets (both $p<0.01$ ). 

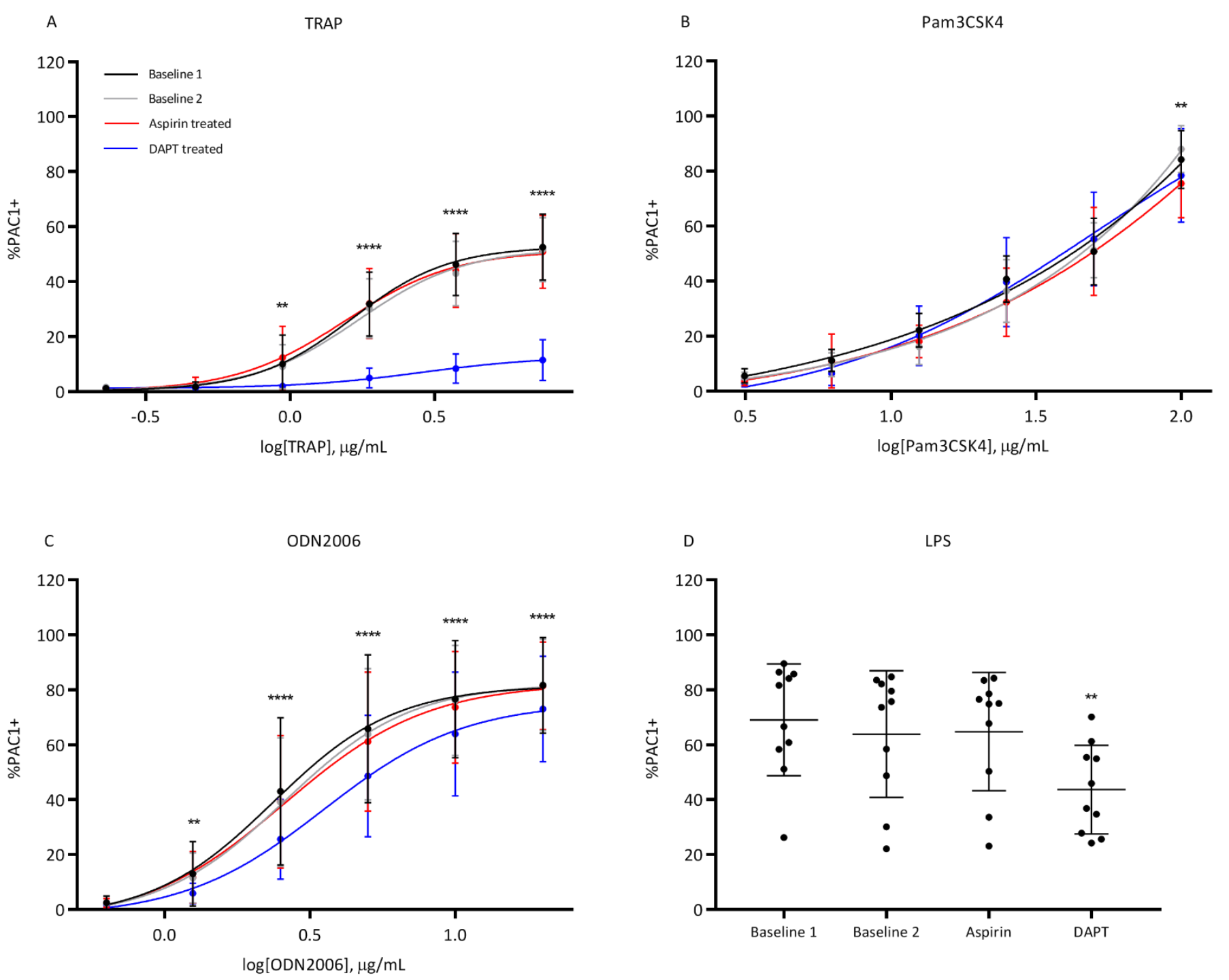

Figure 4.5 Platelet PAC1 expression in response to TLR stimulation is modestly attenuated following DAPT, but not aspirin, administration.

PAC1 positivity was measured in response to TRAP (A), Pam3CSK4 (B), ODN2006 (C) and LPS (D) agonism before (baseline 1 and baseline 2) and after treatment with two anti-platelet drug regimens, aspirin monotherapy and DAPT (a combination of ticagrelor and aspirin). Concentrations of TRAP, Pam3CSK4 and ODN2006 were log transformed. For TRAP, Pam3CSK4 and ODN2006, repeated measures two-way ANOVA was performed and the interaction effect was found to be significant $(p<0.05)$. For LPS, repeated measures one-way ANOVA was found to be significant $(p<0.05)$. Tukey's multiple comparisons tests were performed to detect differences. ${ }^{*}$ indicates a significant difference between the measurements before and after treatment with DAPT. Mean \pm SD for 10 subjects is shown. ${ }^{* *} p<0.01$, $* * * * p<0.0001$. 

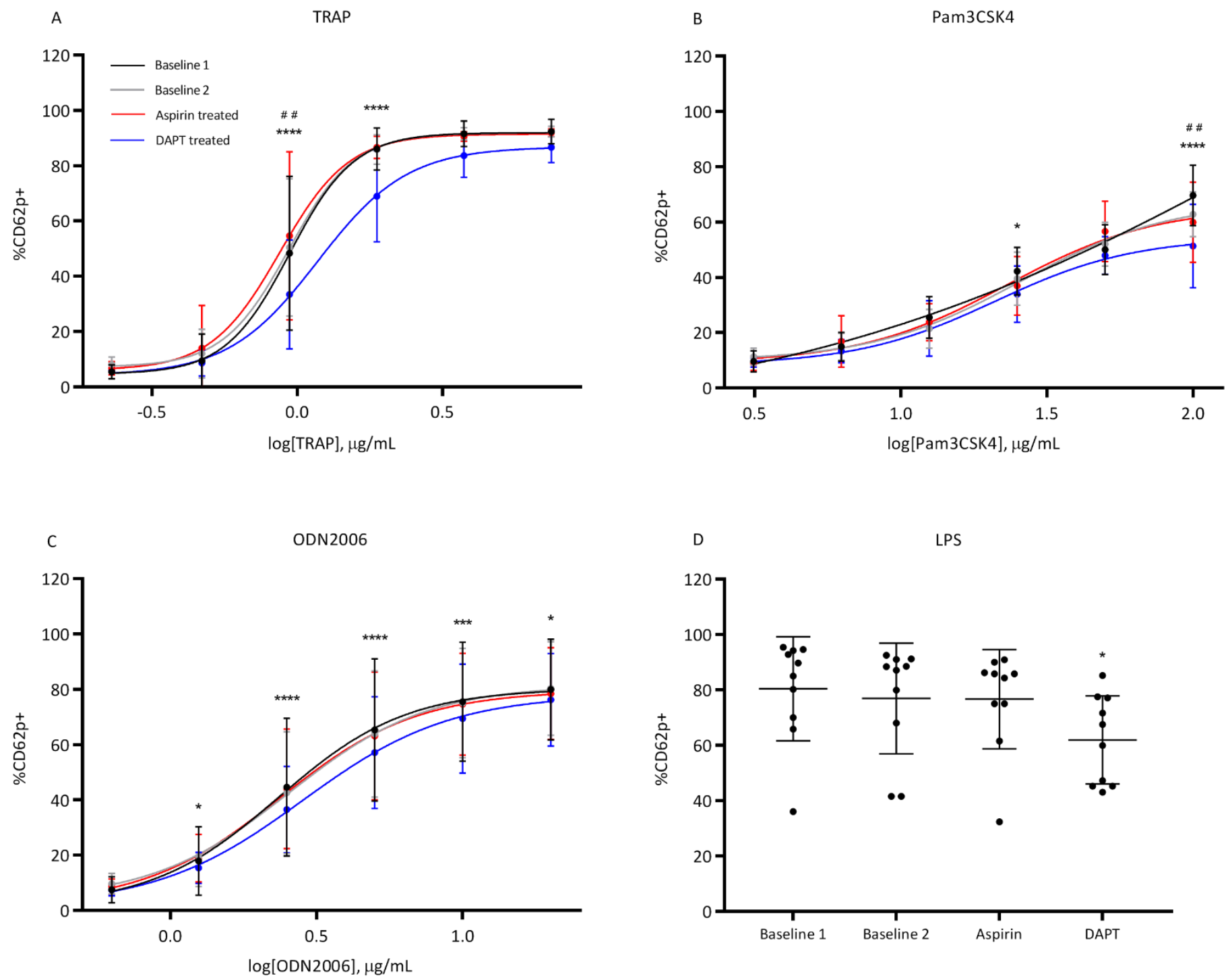

Figure 4.6 CD62p expression is modestly modulated in response to TLR stimulation following aspirin and DAPT administration.

CD62p positivity was measured in response to TRAP (A), Pam3CSK4 (B), ODN2006 (C) and LPS (D) agonism before (baseline 1 and baseline 2) and after treatment with two anti-platelet drug regimens, aspirin monotherapy and DAPT (ticagrelor and aspirin). Concentrations of TRAP, Pam3CSK4 and ODN2006 were log transformed. For TRAP, Pam3CSK4 and ODN2006, repeated measures two-way ANOVA was performed and the interaction effect was found to be significant $(p<0.05)$. For LPS, repeated measures one-way ANOVA was found to be significant $(p<0.05)$. Tukey's multiple comparisons tests were performed to detect differences. * indicates a significant difference between the measurements before and after treatment with DAPT. \# indicates a significant difference between the measurement before and after aspirin monotherapy. Mean \pm SD for 10 subjects is shown. ${ }^{*} p<0.05,{ }^{\#} p<0.01, * * * p<0.001, * * * *$ $p<0.0001$. 


\subsubsection{The effect of anti-platelet therapy on TLR-mediated platelet activation.}

To investigate whether anti-platelet therapy inhibits TLR-mediated platelet activation, PAC1 and CD62p expression was analysed following aspirin monotherapy and following DAPT, a combination of aspirin and ticagrelor, as described in Figure 4.1. All subjects completed the study, reported taking each drug regimen as instructed and did not report any inter-current illness during the study.

\subsubsection{TLR-mediated platelet activation following aspirin therapy.}

Aspirin monotherapy did not have a significant impact on TRAP6-mediated or TLR-mediated platelet activation. The absolute changes of PAC1 and CD62p expression following both drug regimens are given in Table 4.4. Aspirin monotherapy did not change platelet PAC1 expression in response to any concentration of TRAP6 (Figure 4.5A), although CD62p expression was greater in response to a moderate dose of TRAP6 following aspirin monotherapy $(9.1 \%$ absolute increase in CD62p+, p<0.01, Figure 4.6A). Aspirin monotherapy attenuated CD62p-positivity only following incubation with high-dose Pam3CSK4 (absolute reduction of 10.0\%, p<0.01, Figure 4.6B), although aspirin did not affect ODN2006-mediated platelet activation at any dose tested (Figure 4.5C, Figure 4.6C). Expression of both platelet activation markers in response to high-dose LPS was unchanged after aspirin monotherapy (Figure 4.5D, Figure 4.6D). 
Table 4.4 Absolute changes in the percentage of the platelet population that was PAC1+ and CD62p+ following aspirin monotherapy and DAPT.

\begin{tabular}{|c|c|c|c|c|c|}
\hline \multirow[b]{2}{*}{ Agonist } & \multirow[b]{2}{*}{ Conc. $(\mu \mathrm{g} / \mathrm{mL})$} & \multicolumn{2}{|c|}{$\triangle \%$ PAC1+ } & \multicolumn{2}{|c|}{$\Delta \% C D 62 p+$} \\
\hline & & After aspirin & After DAPT & After aspirin & After DAPT \\
\hline \multirow[t]{6}{*}{ TRAP } & 0.2 & -0.2 & 0.3 & -0.2 & -0.8 \\
\hline & 0.5 & 0.3 & -0.3 & 2.9 & -1.8 \\
\hline & 0.9 & 3.2 & $-8.1 * *$ & $9.1 * *$ & $-19.7 * * * *$ \\
\hline & 1.9 & -1.4 & $-23.7 * * * *$ & 0.2 & $-16.4 * * * *$ \\
\hline & 3.8 & -4.0 & $-32.8 * * * *$ & -1.6 & -7.2 \\
\hline & 7.5 & -5.0 & $-37.0 * * * *$ & -1.1 & -5.0 \\
\hline \multirow[t]{6}{*}{ Pam3CSK4 } & 3.1 & -1.2 & -2.5 & -1.2 & -1.2 \\
\hline & 6.3 & 1.4 & -4.2 & 2.4 & -2.3 \\
\hline & 12.5 & 0.3 & -0.7 & 1.4 & -4.2 \\
\hline & 25.0 & -7.9 & 0.0 & -7.0 & $-8.5 *$ \\
\hline & 50.0 & 1.4 & 0.6 & 3.5 & -5.3 \\
\hline & 100.0 & -6.8 & $-11.0 * *$ & $-10.0 * *$ & $-15.3 * * * *$ \\
\hline \multirow[t]{6}{*}{ ODN2006 } & 0.6 & -0.3 & -0.5 & 0.0 & -1.4 \\
\hline & 1.3 & -0.8 & $-6.7 * *$ & 0.9 & $-4.5 *$ \\
\hline & 2.5 & -2.0 & $-15.1 * * * *$ & 1.2 & $-7.6 * * * *$ \\
\hline & 5.0 & -1.4 & $-15.8 * * * *$ & 0.9 & $-7.8 * * * *$ \\
\hline & 10.0 & -0.9 & $-12.1 * * * *$ & 0.9 & $-6.1 * * *$ \\
\hline & 20.0 & -1.0 & $-8.5 * * *$ & 0.8 & $-4.2 *$ \\
\hline LPS & 100.0 & -0.1 & $-22.9 * *$ & -1.1 & $-17.6 *$ \\
\hline
\end{tabular}

Absolute change of each platelet activation marker was calculated as the difference between the before-drug and after-drug measurement. For TRAP, Pam3CSK4 and ODN2006, repeated measures two-way ANOVA was performed and the interaction effect was found to be significant $(p<0.05)$. For LPS, repeated measures one-way ANOVA was found to be significant $(p<0.05)$. Tukey's multiple comparisons tests were performed to detect differences. ${ }^{*} p<0.05,{ }^{* *} p<0.01,{ }^{* * *} p<0.001, * * * * p<0.0001$. 


\subsubsection{TLR-mediated platelet activation following DAPT.}

DAPT inhibited TRAP6-mediated and TLR-mediated platelet activation, to varying degrees. Absolute changes in expression of each platelet activation marker following DAPT are given in Table 4.4. Following DAPT, PAC1 expression was significantly attenuated in response to most doses of TRAP6 (significant absolute reduction of $8.1 \%$ to $37.0 \%$, all $p<0.01$, Figure $4.5 \mathrm{~A}$ ). DAPT inhibited CD62p-positivity only at moderate doses of TRAP6 (reductions of $16.4 \%$ and $19.7 \%$, both $\mathrm{p}<0.0001$, Figure 4.6A). DAPT attenuated both PAC1 (11.0\% reduction, $\mathrm{p}<0.01$, Figure $4.5 \mathrm{~B}$ ) and CD62p expression (15.3\% reduction, $p<0.0001$ ) in response to the highest dose of Pam3CSK4 and also attenuated CD62p expression (8.5\% reduction, $\mathrm{p}<0.05$ ) in response to a moderate dose of Pam3CSK4 (Figure 4.6B). ODN2006-mediated PAC1 and CD62p expression was lowered following DAPT at most doses (PAC1+, reductions of $6.7 \%$ to $15.8 \%$, all $p<0.001$, Figure $4.5 \mathrm{C}$; CD62p+, reductions of $4.2 \%$ to $7.8 \%$, all $p<0.05$, Figure $4.6 \mathrm{C}$ ). After DAPT, expression of both PAC1 and CD62 $p$ was attenuated in response to high-dose LPS. Absolute PAC1 expression was attenuated by 22.9\% ( $p<0.01$, Figure 4.5D) and absolute CD62p expression attenuated by $17.6 \%(p<0.05$, Figure 4.6D) by DAPT. 


\subsection{Discussion.}

Our previous studies have reported dose-dependent and direct platelet activation in response to a TLR2/1 and TLR9 agonist, and direct platelet activation only in response to high-dose TLR4 agonism (see Chapters 2 and 3). These distinct activation patterns are unchanged in AMI patients, despite guideline-recommended treatment with DAPT. Previously, we have not been able to assess TLR-mediated platelet activation in AMI in the absence of DAPT due to early administration of anti-platelet agents upon, or prior to, hospitalization. As such, we were unable to determine how effectively DAPT inhibits this type of platelet activation. This problem was addressed in the current study: we aimed to determine the extent to which aspirin alone or in combination with ticagrelor can inhibit platelet activation in response to TLR2/1, TLR4 and TLR9 stimulation.

We have demonstrated dose-dependent increases in platelet-surface expression of PAC1 and CD62p in response to Pam3CSK4 (a TLR2/1 agonist) and ODN2006 (a TLR9 agonist) as well as in response to high dose LPS (a TLR4 agonist). These results were consistent across both baseline measurements in this study. Aspirin monotherapy had no effect on platelet activation via these TLR pathways or via TRAP. On DAPT, a combination of aspirin and ticagrelor, there was significant reduction in platelet activation in response to TRAP6, seen to a greater extent with PAC1 expression than with CD62p expression. DAPT resulted in a slight inhibition of platelet activation in response to all three TLR agonists, and this inhibition was more modest than the inhibition seen in response to TRAP6. As substantial platelet activation was still observed despite the use of commonly used anti-platelet agents, we suggest that these platelet-TLR pathways represent alternative platelet activation pathways that may contribute to platelet activation post-AMI and despite anti-platelet treatment. From the results of this study, we can conclude that we may expect to see a slight increase in TLR-mediated platelet activation in AMI patients in the absence of anti-platelet therapy. However, anti-platelet therapy was not able to significantly alter these 
platelet activation patterns, and AMI patients are likely to still be at risk of experiencing TLRmediated platelet activation despite optimal and potent anti-platelet therapy.

Aspirin is routinely recommended for both primary and secondary prevention in CAD, and aspirin monotherapy has been shown to reduce recurrent MACE in AMI patients [101]. Aspirin exerts its anti-platelet effect primarily by interfering with the biosynthesis of TXA2 from arachidonic acid via inhibition of COX-1. TXA2 is a potent platelet agonist that can magnify the signal of other platelet agonists [292] and, therefore, aspirin has an ability to prevent amplification of platelet activation initiated via other activation pathways. In this study, aspirin monotherapy did not significantly inhibit TRAP6-mediated or TLR-mediated platelet activation. However, aspirin effectively inhibited platelet aggregation in response to arachidonic acid in each of the healthy subjects, indicating effective inhibition of the target COX-1 receptor. Therefore, we can exclude both non-compliance and aspirin resistance as reasons for observing no effect of aspirin monotherapy on TRAP6- or TLRmediated platelet activation. These finding suggest that aspirin is unable to protect against TRAP6or TLR-mediated platelet activation, and that these activation pathways do not require signalling through the COX-1/TXA2 pathway to elicit potent platelet activation. The platelet-TLR2/1, plateletTLR4 and platelet-TLR9 pathways can provide a pathway for alternative platelet activation despite treatment with aspirin.

DAPT, a combination of aspirin and a P2Y 12 receptor antagonist, inhibits the platelet response to both ADP and COX-1 agonists and is known to reduce the risk of recurrent MACE following AMI when compared to treatment with aspirin alone $[293,294]$. ADP is an important platelet agonist that is released from platelet dense granules and, similar to thromboxane A2, amplifies responses to other agonists [295]. Ticagrelor, a direct-acting and reversible $\mathrm{P}_{2} \mathrm{Y}_{12}$ receptor antagonist, provides more potent and consistent platelet inhibition [296] and superior clinical outcomes [111] when compared to clopidogrel, another commonly used $\mathrm{P}_{2} \mathrm{Y}_{12}$ antagonist. Ticagrelor is now recommended as the first-line anti-platelet agent, along with aspirin, following AMI [94, 104-106]. 
For this reason, we chose to determine the extent to which the combination of aspirin and ticagrelor can inhibit TLR-mediated platelet activation.

Overall, the combination of aspirin and ticagrelor modestly inhibited expression of both platelet activation markers for high-dose LPS, high-dose Pam3CSK4 and several doses of ODN2006. Although statistically significant, the magnitude of this inhibition was relatively small, with a maximum absolute reduction in PAC1 expression of 22.9\% and in CD62p expression of 17.6\%. As aspirin alone was not sufficient to inhibit TLR-mediated platelet activation, we suggest that the driver of the slight inhibition seen with DAPT is due to treatment with ticagrelor. Whilst this type of inhibition may provide some clinical protection, we have shown that incubation with these TLR agonists can still lead to substantial platelet activation despite treatment with DAPT. DAPT effectively inhibited platelet aggregation in response to both arachidonic acid and ADP stimulation, indicating that both of these activation pathways were sufficiently blocked by the antiplatelet agents used in this study. We can conclude that the slight inhibition in TLR-mediated platelet activation seen with DAPT was not due to non-compliance or drug resistance. As a whole, these results suggest that platelets can become activated in response to these TLR agonists despite potent inhibition of the ADP and arachidonic acid pathways. These platelet-TLRs do not require signalling through either the ADP or COX-1/TXA2 pathways to induce potent platelet activation, and DAPT provides incomplete protection against these alternative platelet activation pathways.

DAPT was able to significantly reduce platelet activation in response to TRAP6. More specifically, DAPT substantially attenuated PAC1 expression in response to most doses, and attenuated CD62p expression in response to some doses. This dampening effect on TRAP-mediated platelet activation was greater than for TLR-mediated platelet activation. The differences in this dampening effect seen between PAC1 and CD62p expression indicates differential inhibition of these two distinct platelet activation pathways. These result suggest that DAPT can almost completely block integrin activation (as measured by PAC1 expression). Furthermore, DAPT can 
moderately block platelet degranulation (as pleasured by CD62p expression) at a specific dose range and, above this dose range, inhibition of platelet degranulation is overridden and ontreatment degranulation levels are similar to non-treated levels. Overall, DAPT more effectively inhibits platelet activation in response to TRAP6, a traditional platelet agonist, than platelet activation in response to TLR stimulation.

We have shown that TLR-mediated platelet activation can continue to occur despite treatment with currently used anti-platelet agents. TLRs represent an alternative pathway of platelet activation that is inadequately inhibited by these treatment strategies. Given that all experiments were performed in PRP, in the absence of leukocytes, platelet activation in response to TLR stimulation is likely to represent a direct effect of each TLR agonist on the platelet population. At the patient level, persistent TLR-mediated platelet activation may cause recurrent thrombosis, exacerbate I/R injury and is a potential pathway for recurrent ischaemic events. Both platelets [297-299] and TLRs [178, 180, 300] have been implicated in AMI and in I/R injury, and inhibiting their involvement blunts the resulting post-ischaemic and post-reperfusion inflammatory response. Additionally, increased platelet reactivity has been reported in response to both viral and bacterial threats $[129,130]$, both of which potently stimulate TLRs, and these findings may underlie the association between infection and the incidence of AMI [228, 234]. It is possible that platelet-TLR activation is involved in AMI and recurrent MACE, and current anti-platelet therapies inadequately dampen the platelet-TLR involvement in these pathological processes. 


\subsection{Limitations.}

In this study, we did not assess any other measure of platelet function aside from PAC1 and CD62p expression. However, these two markers provide information on two distinct platelet activation pathways and are commonly used markers to assess platelet activation. We cannot exclude that the TLR agonists used in this study could have off-target effects, although these agonists have been used to assess platelet activation previously [184, 198, 215]. We assessed the platelet response to TLR stimulation in PRP, but these responses were not assessed in whole blood. We, therefore, could not determine the contribution to platelet activation by other cell types that are also likely to be activated by TLR stimulation. However, we aimed to assess the effect of common anti-platelet agents acting directly at the platelet level and the use of PRP for this study satisfied this aim. We cannot determine whether the effect of DAPT with ticagrelor is similar to the effect of DAPT with other $\mathrm{P}_{2} \mathrm{Y}_{12}$ receptor antagonists. However, the use of ticagrelor in this study was appropriate. For example, although clopidogrel is commonly prescribed in New Zealand, this drug has highly variable inter-individual platelet inhibition. Prasugrel is known to have a more consistent inhibitory effect on platelets than clopidogrel, but is not funded for routine use in the treatment of AMI patients in New Zealand. In contrast, ticagrelor is funded and well-prescribed in New Zealand and has a consistent inhibitory effect on platelets and was, therefore, used in this study. 


\subsection{Conclusion.}

In conclusion, we have shown that stimulation with TLR2/1, TLR4 and TLR9 agonists can result in substantial platelet activation. Furthermore, aspirin monotherapy had no effect on platelet activation in response to stimulation with any TLR agonist tested and DAPT (a combination of aspirin and ticagrelor) only modestly inhibited these platelet activation patterns. These results demonstrate that currently available anti-platelet therapies cannot adequately protect against activation of these platelet-TLR pathways. We suggest that platelet-TLRs represent intact ontreatment platelet activation pathways, and that these pathways may contribute to recurrent platelet activation in the setting of AMI. 
5 Chapter 5. Platelets modulate leukocyte function in response to TLR stimulation. 


\subsection{Introduction.}

Aside from their well-established role in thrombosis, platelets are increasingly recognized as participants in vascular inflammation primarily by their interaction with leukocytes [8]. Platelets are likely to mediate inflammation, at least in part, via their TLRs and readily aggregate with a number of leukocyte subsets in response to TLR stimulation $[19,184]$.

In Chapter 3, we demonstrated that platelets have very distinct activation patterns and PMAgg patterns in response to a range of TLR agonists [301]. In particular, we demonstrated that platelets become directly and dose-dependently activated by a TLR2/1 agonist, but only become directly activated in response to high-dose TLR4 agonism and remain quiescent when directly stimulated with a TLR2/6 agonist. Similarly, platelets dose-dependently aggregate with monocytes in response to TLR2/1 stimulation, and variably form these heterotypic aggregates in response to TLR2/6 stimulation, but not in response to TLR4 stimulation. In summary, some TLR agonists can induce both platelet activation and PMAgg although other TLR agonists are only able to induce PMAgg. On the basis of these results, we suggest that both the thrombotic and inflammatory responses of platelets are TLR agonist-specific.

Other than examining platelet aggregation with monocytes, we did not fully characterize the inflammatory responses of platelets in Chapter 3. However, the data from this study suggests that platelets are likely to influence leukocyte function in a TLR agonist-specific manner. The platelet involvement in shaping leukocyte responses has been studied previously, although there are gaps in the current literature. The platelet effect on leukocyte function has mainly been characterized in PBMCs and in response to LPS or in models of sepsis. Thus, there is a lack of research examining how the effect of platelets differs in response to a range of other TLR agonists. Furthermore, we do not know whether platelets exert differential effects on leukocyte subsets in response to TLR agonism. There is also uncertainty as to whether platelets drive a pro-inflammatory or an antiinflammatory leukocyte response to TLR agonism. Interestingly, the role of platelets in mediating 
immune responses to infection is two-sided: platelets have been viewed predominantly as proinflammatory [21], although ever-accumulating evidence suggests that platelets can also limit leukocyte effector functions to prevent rampant inflammation [22].

On the basis of our previous results from Chapter 3, we hypothesize that platelets will modify the responses of leukocyte subsets in a TLR agonist-specific manner. We further hypothesize that platelets will drive a more anti-inflammatory response in leukocytes. To address this hypothesis, we aimed:

1. To determine the extent to which platelets modulate PBMC (monocyte, $T$ cell) and granulocyte (neutrophil) responses in vitro to TLR4, TLR2/1, and TLR2/6 agonism in healthy subjects. 


\subsection{Materials and methods.}

\subsubsection{Subject recruitment and blood sampling.}

Blood was collected via venepuncture from 10 healthy subjects (5 male, $29 \pm 5$ years) with no known cardiovascular disease, diabetes mellitus, or inflammatory or platelet function disorder. These subjects were recruited as described in Section 2.2.1.1. Blood was collected into hirudinanticoagulated tubes (Dynabyte) and EDTA-anticoagulated tubes (Becton Dickinson).

\subsubsection{Isolation of platelets and leukocytes.}

Hirudin-anticoagulated blood was centrifuged at $200 \times$ g for 12 minutes to produce PRP or at 1500 $\mathrm{x} g$ for 12 minutes to produce PPP. PRP was diluted in PBS to $2.5 \times 10^{8}$ platelets $/ \mathrm{mL}$, and PPP was diluted in PBS in the same ratio as PRP. EDTA-anticoagulated blood was layered over Polymorphprep (Axis-Shield, Dundee, United Kingdom), centrifuged at 650 x g for 30 minutes, and the PBMC and granulocyte layers were isolated and washed in PBS. To lyse erythrocytes, PBMCs were resuspended in erythrocyte lysis buffer (Qiagen) and granulocytes were resuspended in icecold water followed by the addition of PBS in excess. Finally, both cell suspensions were washed and resuspended in isolation buffer (0.1\% BSA, 2 mM EDTA in PBS, pH 7.4; see Appendix 2).

\subsubsection{Platelet depletion from leukocytes.}

Each leukocyte fraction were incubated with $10 \mu \mathrm{g}$ anti-human CD42b conjugated to biotin (clone AK2; Thermo Fisher Scientific) for 30 minutes at $4^{\circ} \mathrm{C}$, washed and finally incubated with $100 \mu \mathrm{L}$ Biotin Binder Dynabeads (Thermo Fisher Scientific) for 30 minutes at $4^{\circ} \mathrm{C}$. Platelets were then removed by magnetic separation. Alongside CD42a, CD42b is a surface receptor that uniquely 
identifies the platelet population. Leukocytes were resuspended in cell culture media (10\% FCS, 2 mM L-glutamate, $100 \mathrm{U} / \mathrm{mL}$ penicillin, $100 \mu \mathrm{g} / \mathrm{mL}$ streptomycin, $0.01 \mathrm{M} \mathrm{HEPES}$ buffer, $0.1 \% \beta$ mercaptoethanol, $0.01 \mathrm{nM}$ non-essential amino acids; see Appendix 2) to $1 \times 10^{6}$ cells $/ \mathrm{mL}$, and kept on ice for 60 minutes prior to stimulation.

\subsubsection{In vitro stimulation of PBMCs and granulocytes.}

A portion of PBMCs and granulocytes were co-cultured with PRP in a leukocyte:platelet ratio of 1:250. As a control condition, PPP was added to a separate portion of leukocytes, and these leukocytes were cultured alone. All cells were kept at $37^{\circ} \mathrm{C} / 5 \% \mathrm{CO}_{2}$ for 60 minutes prior to TLR stimulation. Leukocytes \pm platelets were then either left unstimulated or stimulated with 1 and 100 ng/mL of LPS (TLR4 agonist; Enzo Life Sciences), Pam3CSK4 (TLR2/1 agonist; Tocris Bioscience), and FSL-1 (TLR2/6 agonist; Santa Cruz Biotechnology). Granulocytes were stimulated for 4 hours and PBMCs were stimulated for 24 hours at $37^{\circ} \mathrm{C} / 5 \% \mathrm{CO}_{2}$.

\subsubsection{Flow cytometry.}

Following TLR stimulation, all samples were centrifuged at $400 \times \mathrm{g}$ for 12 minutes, and the cell culture supernatant was collected and stored at $-80^{\circ} \mathrm{C}$. Leukocytes \pm platelets were resuspended in Fixable Viability Stain (FVS) 620 (Becton Dickinson) diluted in PBS and incubated for 15 minutes. Samples were washed twice in staining buffer and resuspended in Fc receptor blocking solution (BioLegend) diluted in staining buffer. Granulocytes \pm platelets were incubated with anti-CD16BV421 (clone 3G8), anti-CD45-PE/Cy7 (clone HI30), and anti-CD66b-BB515 (clone G10F5). PBMCs \pm platelets were incubated with anti-CD64-BV421 (clone 10.1), anti-CD3-BV510 (clone UCHT1), anti-CD8-AF647 (clone RPA-T8), anti-CD4-APC/H7 (clone RPA-T4), anti-HLA-DR-PE/Cy7 (clone G466), and anti-CD38-BB515 (clone HIT2). The panel design for this experiment is outlined in Table 5.1. All antibodies were sourced from Becton Dickinson. Antibodies were diluted in staining buffer 
and all samples were stained for 50 minutes at $4^{\circ} \mathrm{C}$. Appropriate isotype controls, all sourced from Becton Dickinson, were run in parallel. Antibody-stained leukocytes \pm platelets were fixed with $1 \%$ paraformaldehyde, centrifuged and finally resuspended in staining buffer. All samples were analysed on a FACSCanto II flow cytometer (Becton Dickinson). Flow cytometric data was analysed using FlowJo software (v10.0.7; Treestar). Live cell sub-populations were identified by their FVS620-negativity, and by their characteristic forward and side scatter properties. The geometric MFI of CD66b, HLA-DR and CD38 was used to determine CD16+ neutrophil activation, CD64+ monocyte activation and CD4+/CD8+ T cell activation, respectively. Each cell type was identified as outlined in Figure 5.1, Figure 5.2 and Figure 5.3. A representative plot of each activation marker is given in Figure 5.4 .

Table 5.1 Panel design for assessing leukocyte activation in response to TLR agonism.

\begin{tabular}{|c|c|c|c|}
\hline & $\begin{array}{l}\text { Laser/channel } \\
\text { (fluorophore) }\end{array}$ & $\begin{array}{l}\text { Antibody target } \\
\text { (clone) }\end{array}$ & $\begin{array}{l}\text { Concentration in final staining } \\
\text { volume (final dilution) }\end{array}$ \\
\hline PBMC panel & $\begin{array}{l}\text { Blue/Red } \\
\text { Blue/NIR (PE/Cy7) } \\
\text { Blue/Green (BB515) } \\
\text { Violet/Blue (BV421) } \\
\text { Violet/Green (BV510) } \\
\text { Red/Red2 (AF647) } \\
\text { Red/NIR2 (APC/H7) }\end{array}$ & $\begin{array}{l}\text { FVS620 } \\
\text { HLA-DR (G46-6) } \\
\text { CD38 (HIT2) } \\
\text { CD64 (10.1) } \\
\text { CD3 (UCHT1) } \\
\text { CD8 (RPA-T8) } \\
\text { CD4 (RPA-T4) }\end{array}$ & $\begin{array}{l}\text { ( } 1 \text { in } 2000) \\
0.50 \mu \mathrm{g} / \mathrm{mL} \text { (1 in } 50) \\
4.00 \mu \mathrm{g} / \mathrm{mL} \text { (1 in } 50) \\
2.00 \mu \mathrm{g} / \mathrm{mL} \text { (1 in } 100) \\
1.00 \mu \mathrm{g} / \mathrm{mL} \text { (1 in } 50) \\
0.06 \mu \mathrm{g} / \mathrm{mL} \text { (1 in } 400) \\
0.50 \mu \mathrm{g} / \mathrm{mL} \text { (1 in } 25)\end{array}$ \\
\hline Granulocyte panel & $\begin{array}{l}\text { Blue/Red } \\
\text { Blue/NIR (PE/Cy7) } \\
\text { Blue/Green (BB515) } \\
\text { Violet/Blue (BV421) }\end{array}$ & $\begin{array}{l}\text { FVS620 } \\
\text { CD45 (HI30) } \\
\text { CD66b (G10F5) } \\
\text { CD16 (3G8) }\end{array}$ & $\begin{array}{l}\text { (1 in } 2000) \\
1.00 \mu \mathrm{g} / \mathrm{mL}(1 \text { in } 50) \\
2.00 \mu \mathrm{g} / \mathrm{mL}(1 \text { in } 50) \\
0.25 \mu \mathrm{g} / \mathrm{mL}(1 \text { in } 400)\end{array}$ \\
\hline
\end{tabular}

A list of all antibody formats, clones, isotypes and manufacturers can be found in Appendix 1. 

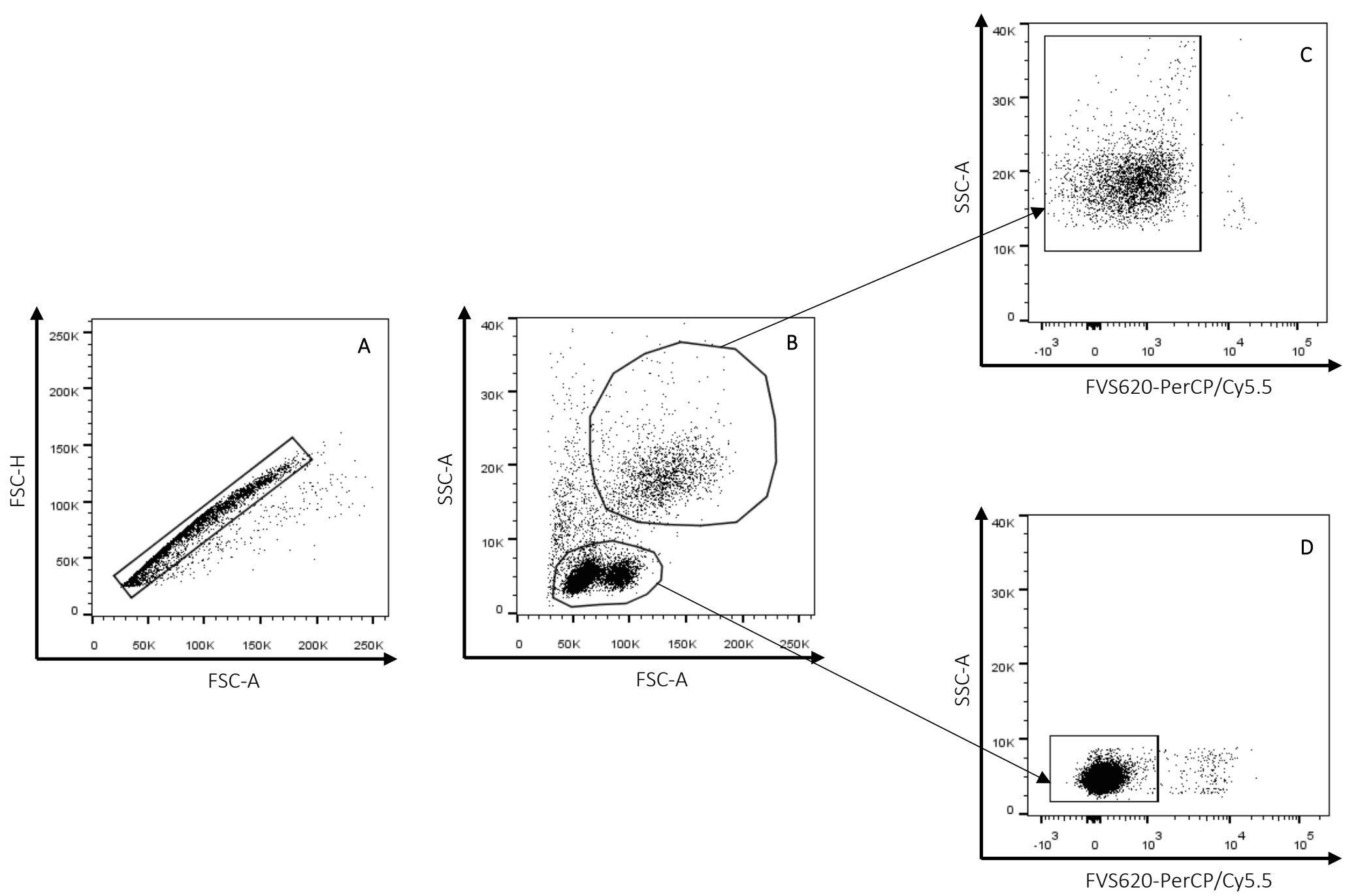

\section{Figure 5.1 Live monocytes and lymphocytes were identified from PBMCs.}

Single cells were gated on (A). Monocytes (top, right gate drawn) and lymphocytes (bottom, left gate) were identified by their forward and side scatter properties (B). For each of these populations, live FVS620-negative cells were gated on (C \& D). 

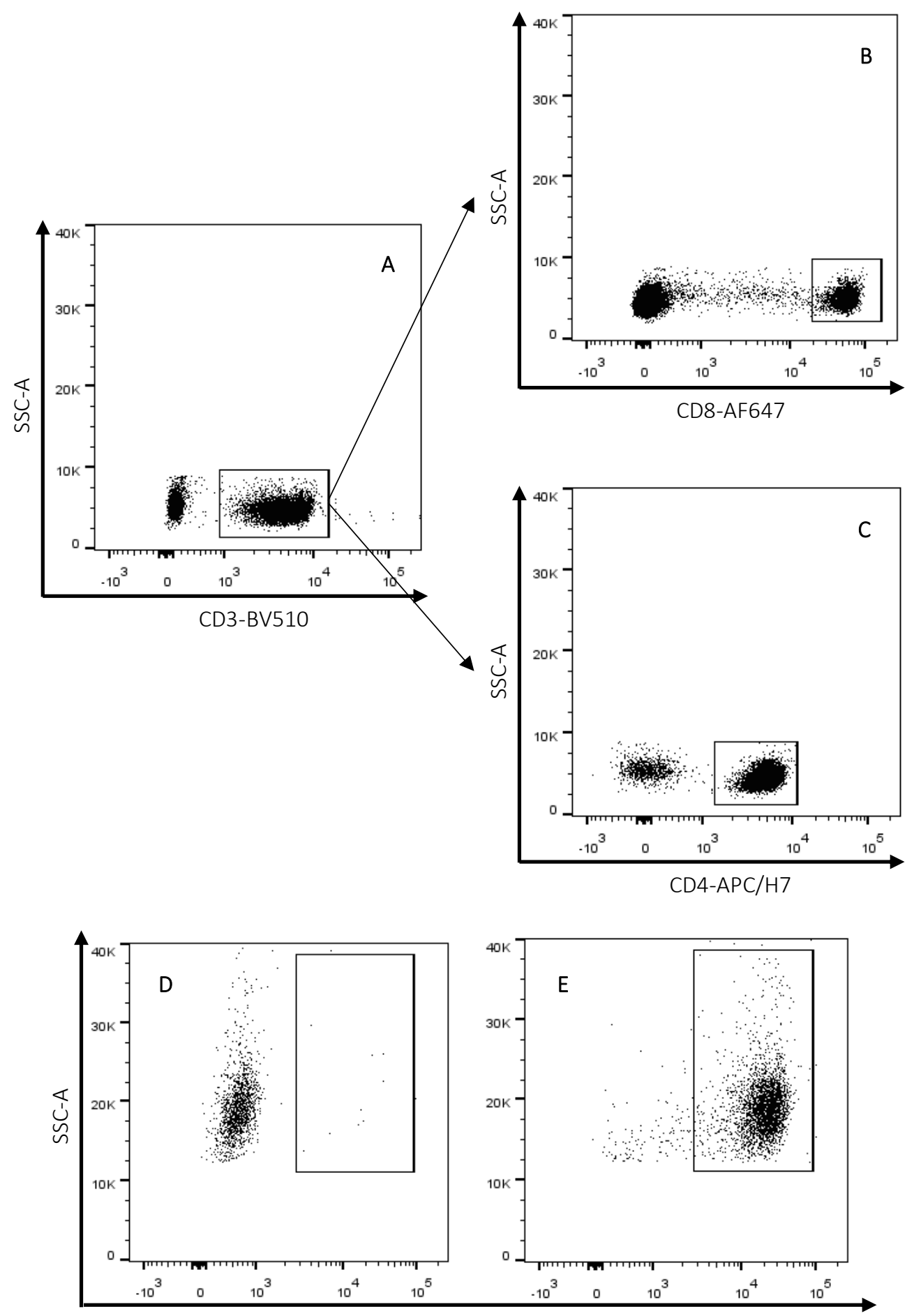

CD64-BV421

Figure 5.2 CD64+ monocytes and CD4+ and CD8+ T cells were identified from PBMCs.

$C D 3+T$ cells were identified (A) and, further, $C D 8+T$ cells (B) and CD4+ T cells (C) were identified. CD64+ monocytes were identified (E) against the isotype control (D). 

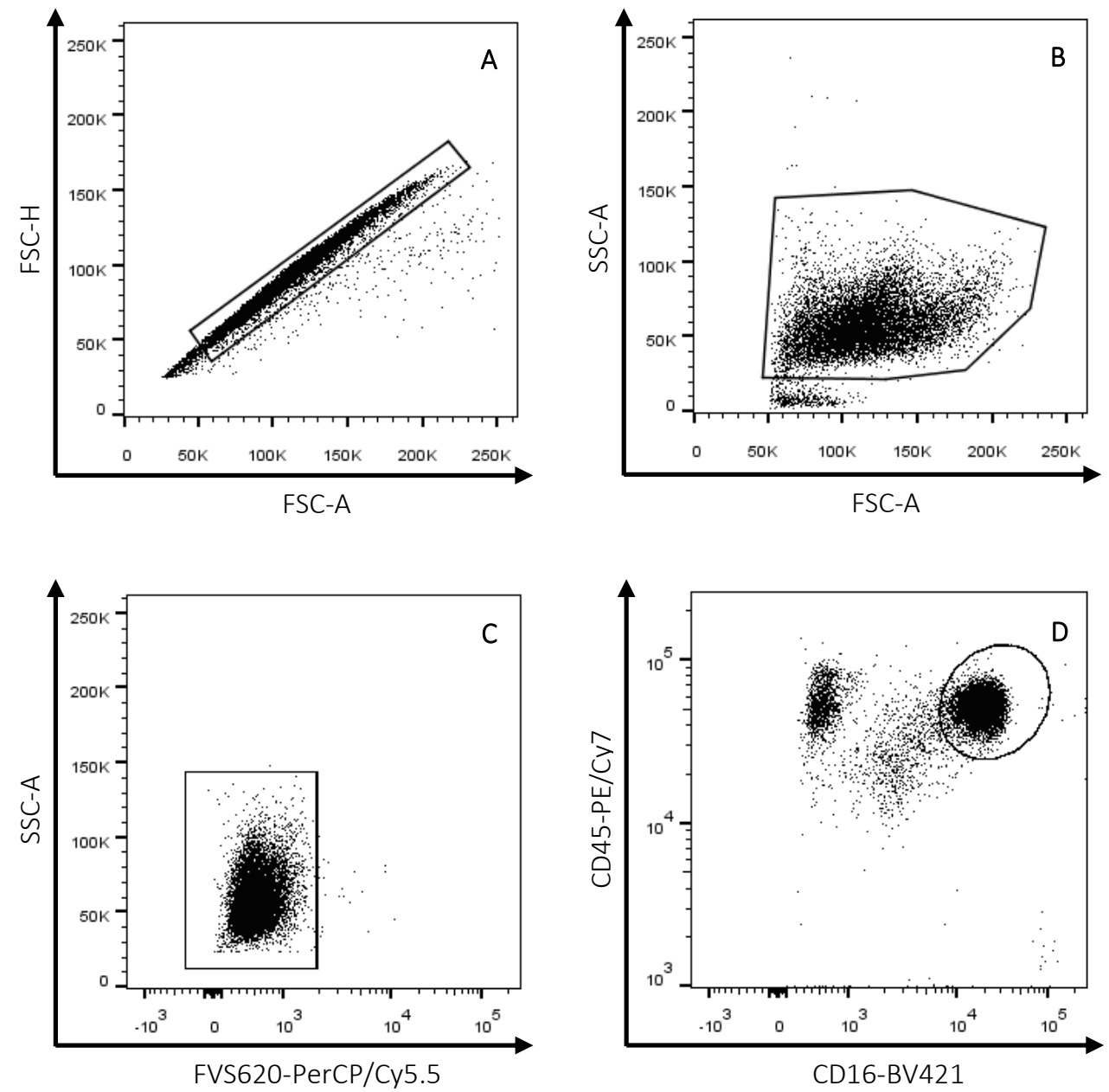

Figure 5.3 Live CD16+ neutrophils were identified from granulocytes.

Single cells were gated on (A). Granulocytes were identified by their characteristic forward and side scatter profile (B), and live granulocytes were gated on (C). Neutrophils were further identified by CD45 expression and CD16-positivity (D). 

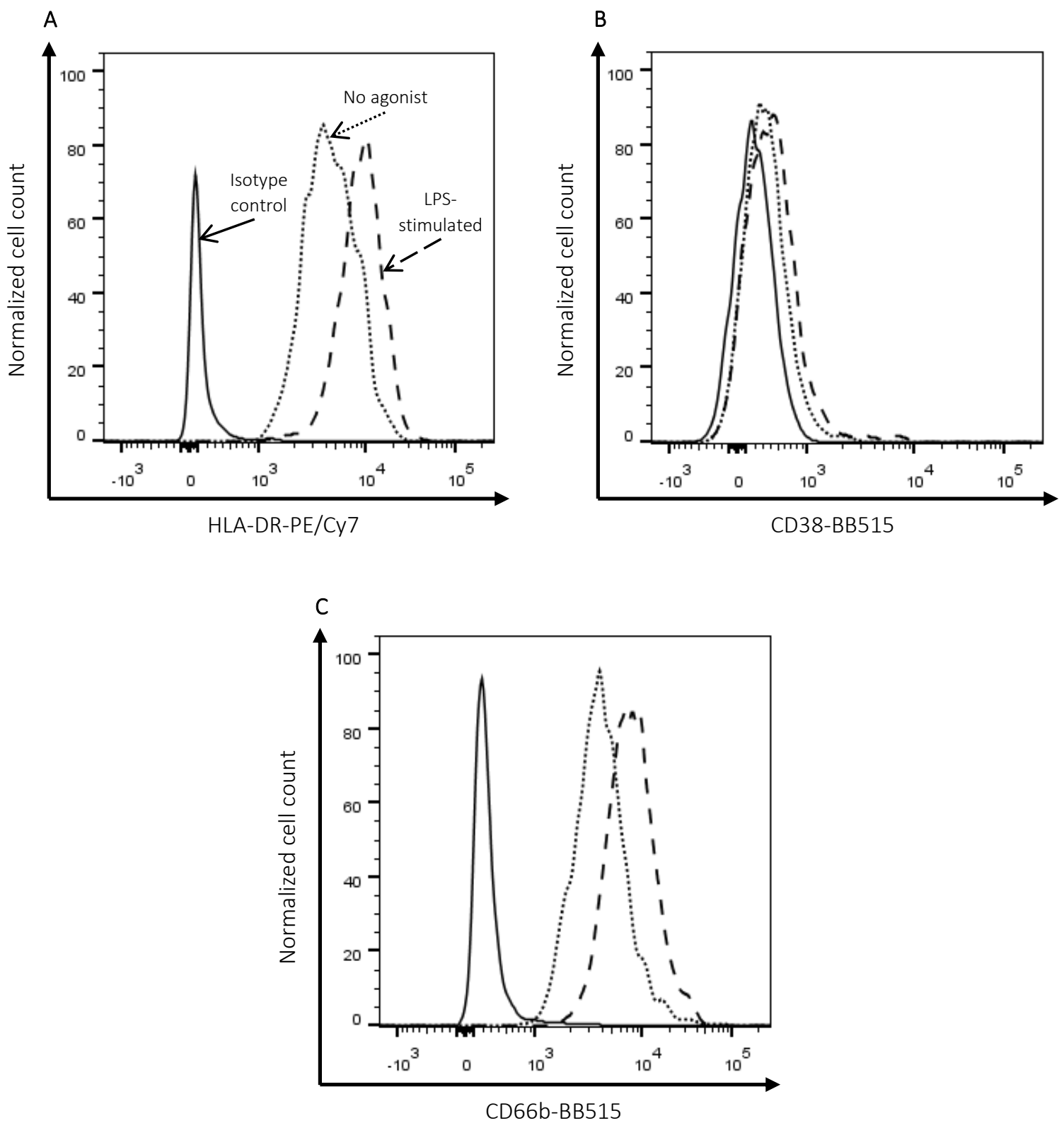

Figure 5.4 Representative plots of leukocyte activation markers.

Representative plots of monocyte HLA-DR expression (A), T cell CD38 expression (B) and neutrophil CD66b expression (C) are shown for unstimulated and LPS (100 ng/mL)-stimulated conditions. 


\subsubsection{Analysis of cell culture supernatant.}

The concentration of elastase in granulocyte cell culture supernatant was measured using a human polymorphonuclear elastase ELISA kit (Abcam, Cambridge, United Kingdom), as per the manufacturer's instructions. The concentration of the following cytokines and chemokines in PBMC cell culture supernatant was measured using a Luminex multiplex assay (R\&D Systems, Minneapolis, MN, USA), as per the manufacturer's instructions: tumour necrosis factor (TNF)- $\alpha$, interleukin (IL)-6, IL-10, macrophage inflammatory protein (MIP)-1 $\beta$.

\subsubsection{Statistical analysis.}

We did not have sufficient data on the variance of the measurements studied here to properly estimate an appropriate sample size. Therefore, we empirically chose to recruit 10 healthy subjects to determine the effect of platelets on leukocyte responses to TLR stimulation. For examining baseline (- platelets) leukocyte activation in response to TLR stimulation, differences in raw measurements were examined using one-way ANOVA with post-hoc Sidak's multiple comparisons tests. For analysing the effect of platelets on the leukocyte response to TLR stimulation, each baseline (- platelets) leukocyte only measurement was normalized to 1 . Each

platelet co-culture (+ platelets) measurement was then reported as a relative change, compared to the paired leukocyte only measurement (+ platelets measurement/- platelets measurement). Differences between these measurements were examined using paired t-tests. All statistical analyses were performed using GraphPad Prism 7 (GraphPad Software Inc.). 


\subsection{Results.}

\subsubsection{Platelets reduce neutrophil CD66b expression in response to TLR stimulation.}

Considering the baseline (- platelets) TLR stimulation results, we demonstrated an increase in neutrophil CD66b expression in response to both a low-dose $(1 \mathrm{ng} / \mathrm{mL})$ and a high-dose (100 $\mathrm{ng} / \mathrm{mL}$ ) of each TLR agonist used in this study. These baseline CD66b expression levels are given in Table 5.2 and, in response to TLR stimulation, CD66b expression is presented as a percentage increase from unstimulated cultures. There was an increase in CD66b expression, which was similar, in response to both low-dose (57\% increase from unstimulated) and high-dose LPS (44\% increase; both $\mathrm{p}<0.01$ ). Neutrophil CD66b expression was also significantly and dose-dependently elevated in response to Pam3CSK4 (41\% and $127 \%$ increases, both $p<0.05)$ and FSL-1 (58\% and $115 \%$ increases, both $\mathrm{p}<0.01)$.

Considering the platelet co-culture results, the addition of platelets did not reduce CD66b expression in unstimulated neutrophil cultures but, interestingly, platelets differentially attenuated neutrophil activation patterns in response to all three TLR agonists. These changes in CD66b expression, as measured by MFI, with platelet co-culture (+ platelets) are given in Figure 5.5A. The relative changes in CD66b expression with co-culture are also given in Figure 5.5B and Table 5.3, and are reported below.

The increase in CD66b expression in response to low-dose LPS was attenuated by $11 \%(p<0.05)$ with platelet co-culture, but was not significantly changed in response to high-dose LPS (relative reduction of $8 \%, p=0.06)$. With platelets, the increase in CD66b expression was attenuated by $9 \%$ at low-dose Pam3CSK4 ( $p=0.09)$ and by $15 \%$ at high-dose Pam3CSK4 $(p<0.05)$ and the increase in response to FSL-1 stimulation was attenuated by $19 \%$ at a low dose and $14 \%$ at a high dose (both $p<0.001)$. 
Table 5.2 Baseline (- platelets) geometric MFI of leukocyte activation markers in response to TLR agonism, compared to unstimulated cultures.

\begin{tabular}{|c|c|c|c|c|c|c|c|c|c|}
\hline \multirow[b]{2}{*}{ Agonist } & \multirow[b]{2}{*}{$\mathrm{ng} / \mathrm{mL}$} & \multicolumn{2}{|c|}{ Neutrophil CD66b } & \multicolumn{2}{|l|}{ Monocyte HLA-DR } & \multicolumn{2}{|c|}{ CD8+ T cell CD38 } & \multicolumn{2}{|c|}{ CD4+ T cell CD38 } \\
\hline & & Absolute MFI & $\begin{array}{l}\% \text { increase } \\
\text { from US }\end{array}$ & Absolute MFI & $\begin{array}{l}\text { \% increase } \\
\text { from US }\end{array}$ & Absolute MFI & $\begin{array}{l}\text { \% increase } \\
\text { from US }\end{array}$ & Absolute MFI & $\begin{array}{l}\% \text { increase } \\
\text { from US }\end{array}$ \\
\hline US & -- & 3142 (988) & -- & 2776 (1689) & -- & $222(95)$ & -- & $335(143)$ & -- \\
\hline \multirow[t]{2}{*}{ LPS } & 1 & $4926(1239)^{* *}$ & $57 \%$ & 5011 (1919) & $81 \%$ & $308(108)^{* * * *}$ & $39 \%$ & $567(158)^{* * * *}$ & $69 \%$ \\
\hline & 100 & $4528(1353)^{* * *}$ & $44 \%$ & $4803(1858)^{*}$ & $73 \%$ & $314(110)^{* * * *}$ & $41 \%$ & $580(167)^{* * * *}$ & $73 \%$ \\
\hline \multirow[t]{2}{*}{ Pam3CSK4 } & 1 & $4440(1375)^{*}$ & $41 \%$ & $4675(1579)^{* * * *}$ & $68 \%$ & $272(105)^{* * * *}$ & $23 \%$ & $530(165)^{* * * *}$ & $58 \%$ \\
\hline & 100 & $7118(2653)^{* *}$ & $127 \%$ & $4763(1693)^{*}$ & $72 \%$ & $294(109)^{* * * *}$ & $32 \%$ & $578(171)^{* * * *}$ & $73 \%$ \\
\hline \multirow[t]{2}{*}{ FSL-1 } & 1 & $4958(1431)^{* *}$ & $58 \%$ & $4299(1452)^{* *}$ & $55 \%$ & $259(99)^{* * *}$ & $17 \%$ & $503(141)^{* * * *}$ & $50 \%$ \\
\hline & 100 & $6752(2675)^{* *}$ & $115 \%$ & $4265(1433)^{* *}$ & $54 \%$ & $266(86)^{* *}$ & $20 \%$ & $513(127)^{* * * *}$ & $53 \%$ \\
\hline
\end{tabular}


Neutrophil activation

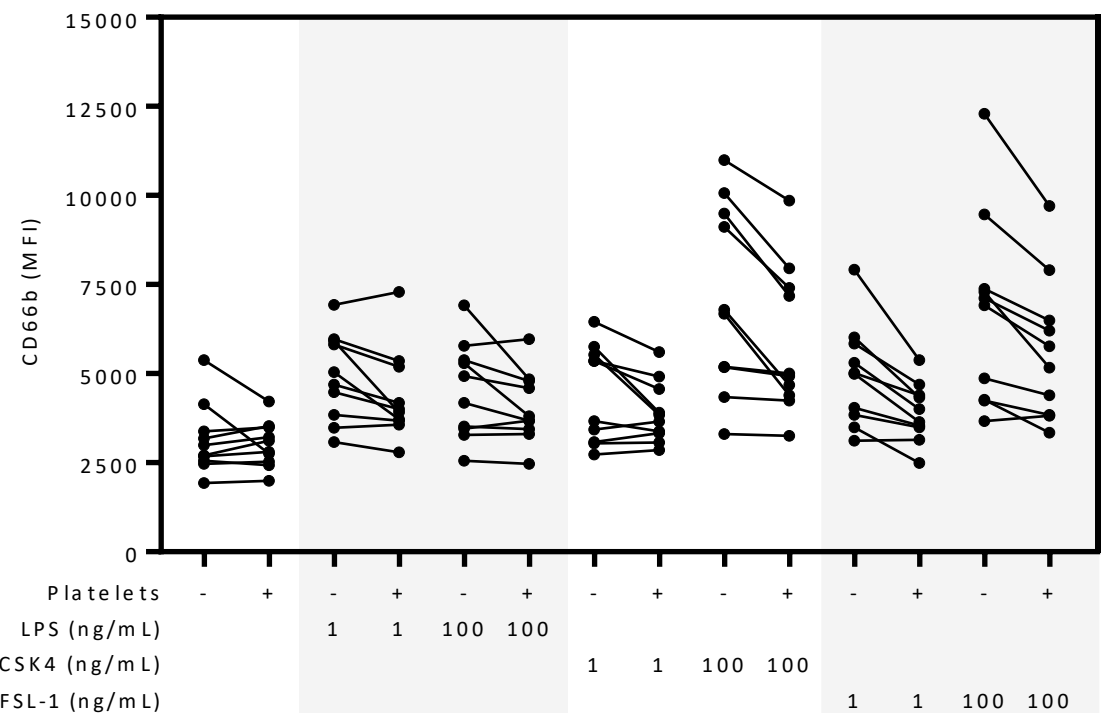

B

Neutrophil activation

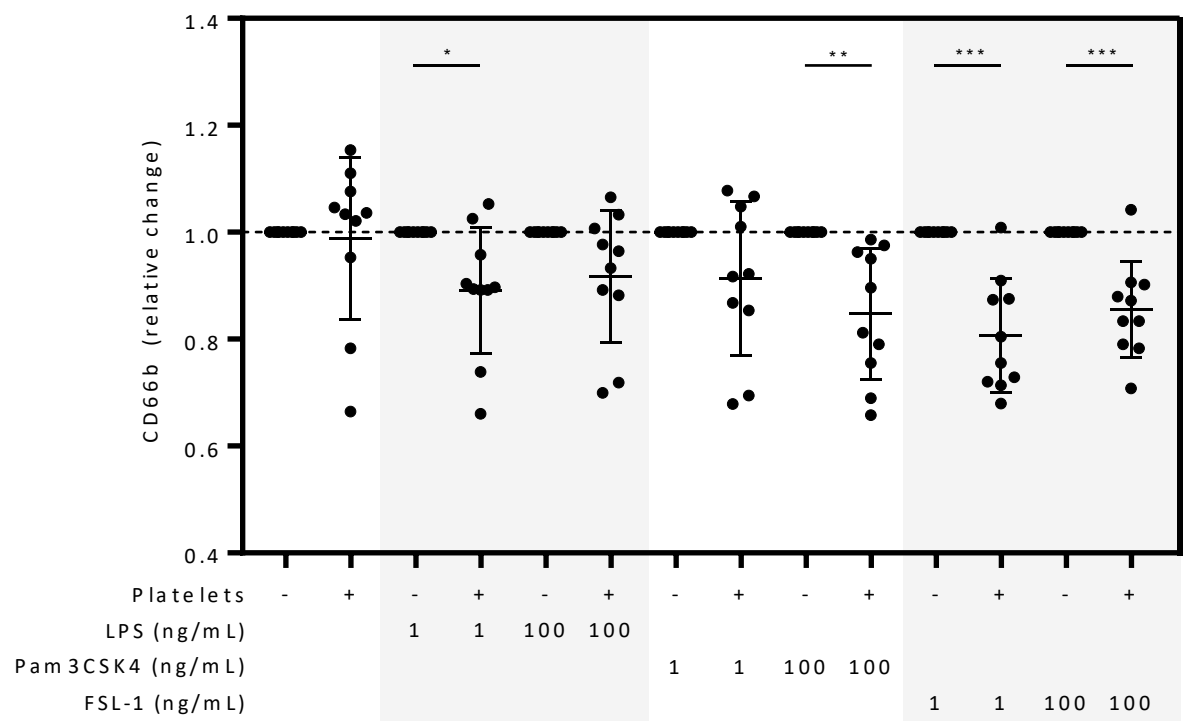

Figure 5.5 In response to TLR stimulation, platelets modulate expression of neutrophil CD66b expression.

Neutrophil CD66b expression was measured by MFI without platelets (- platelets) and in co-culture (+ platelets) with no stimulation and in response to 1 and $100 \mathrm{ng} / \mathrm{mL}$ LPS, Pam3CSK4 and FSL-1 (A). For each co-culture measurement, $\mathrm{CD66b}$ expression was calculated as a relative change (B). Differences between these relative change measurements were examined using paired t-tests. ${ }^{*} p<0.05,{ }^{* *} p<0.01,{ }^{* * *} p<0.001$. 
Table 5.3 Relative change in leukocyte activation markers in leukocyte-platelet co-culture (+ platelets) and following TLR stimulation.

\begin{tabular}{|l|l|l|l|l|l|}
\cline { 3 - 6 } \multicolumn{2}{l|}{} & \multicolumn{4}{|c|}{ platelets $^{\#}$} \\
\hline Agonist & $n g / m L$ & Neutrophil CD66b & Monocyte HLA-DR & CD8+ T cell CD38 & CD4+ T cell CD38 \\
\hline US & -- & $0.99(0.15)$ & $0.97(0.09)$ & $1.06(0.12)$ & $0.98(0.03)$ \\
PPS & 1 & $0.89(0.12)^{*}$ & $0.95(0.06)^{*}$ & $1.04(0.06)$ & $0.99(0.03)$ \\
& 100 & $0.92(0.12)$ & $0.96(0.06)$ & $1.04(0.12)$ & $0.98(0.04)$ \\
& 1 & $0.91(0.14)$ & $0.99(0.10)$ & $1.05(0.07)^{*}$ & $0.99(0.04)$ \\
& 100 & $0.85(0.12)^{* *}$ & $0.97(0.08)$ & $1.04(0.09)$ & $1.00(0.05)$ \\
& 1 & $0.81(0.11)^{* * *}$ & $0.93(0.10)$ & $1.07(0.10)$ & $1.00(0.03)$ \\
& 100 & $0.86(0.09)^{* * *}$ & $0.97(0.07)$ & $1.08(0.12)^{*}$ & $0.99(0.03)$ \\
\hline
\end{tabular}

\#all leukocyte only measurements were normalized to 1, and all co-culture (+ platelets) measurements were compared to this normalized response and reported as relative change. Differences between these measurements were examined by paired t-tests. ${ }^{*} p<0.05, * * p<0.01, * * * p<0.001$. 


\subsubsection{Platelets modestly reduce monocyte HLA-DR expression following low-dose LPS.}

At baseline (in the absence of platelets) and compared to unstimulated monocytes, expression of HLA-DR was elevated in response to high-dose LPS ( $73 \%$ increase from unstimulated, $p<0.05)$ and in response to low-dose and high-dose Pam3CSK4 (68\% and 72\% increases, both $p<0.05$ ) and FSL1 (55\% and 54\% increases, both $p<0.01$ ) (Table 5.2). Monocyte HLA-DR expression was numerically, although not significantly, higher following incubation with low-dose LPS (81\% increase, $p=0.08)$. We saw substantial inter-individual variation in HLA-DR expression in this study, which may explain why this change was not detected as significant. For each TLR agonist, the change in HLA-DR expression was relatively similar across both doses.

The raw and relative changes in monocyte HLA-DR expression following co-culture with platelets are given in Figure 5.6A and Figure 5.6B, respectively. When examining relative change, platelet co-culture significantly, but modestly, decreased HLA-DR expression in response to low-dose LPS by $5 \%(p<0.05$, also given in Table 5.3). Monocyte HLA-DR expression was not changed in the presence of platelets under unstimulated conditions or in response to high-dose LPS, or any dose of Pam3CSK4 or FSL-1. 

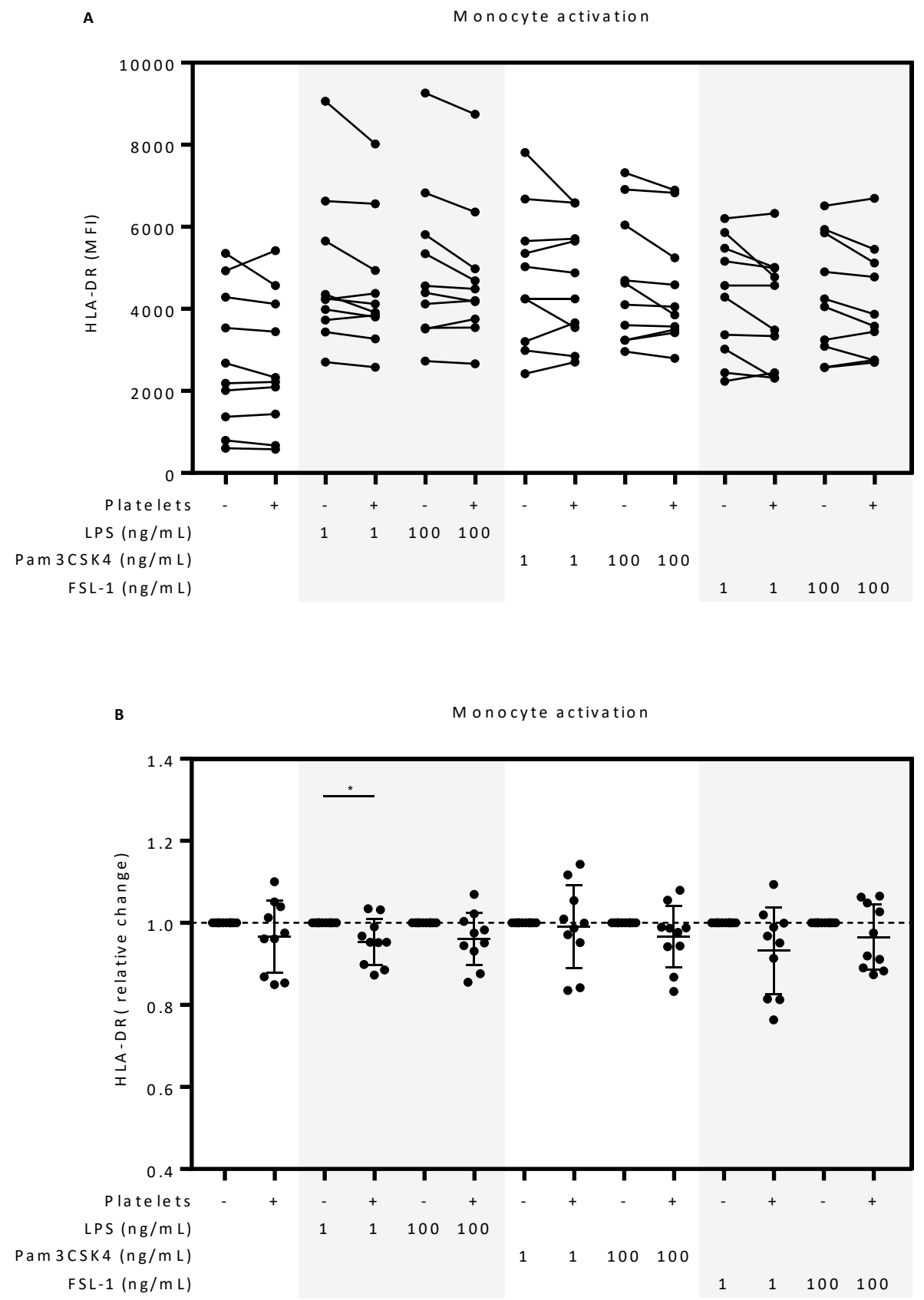

Figure 5.6 Platelets modestly modulate monocyte HLA-DR expression following low-dose LPS stimulation.

Monocyte HLA-DR expression was measured by MFI with (+) and without (-) platelets with no stimulation and in response to 1 and $100 \mathrm{ng} / \mathrm{mL}$ LPS, Pam3CSK4 and FSL-1 (A). For each co-culture measurement, HLA-DR expression was calculated as a relative change (B). Differences between these relative change measurements were examined using paired t-tests. ${ }^{*} p<0.05$. 


\subsubsection{The addition of platelets modulates CD8+ T cell, but not CD4+ T cell, CD38 expression in response to TLR stimulation.}

CD38 expression was used in this study to assess T cell activation, and has been used previously to assess T cell responses to TLR agonism and in the absence of T cell receptor (TCR) engagement $[302,303]$. This activation marker is also recommended for the identification of activated T cells by the Human Immunology Project [304]. For CD8+ T cells at baseline (- platelets), CD38 expression increased in response to all TLR agonists tested (Table 5.2). CD38 expression increased following stimulation with low-dose and high-dose LPS (39\% and $41 \%$ increases, both $p<0.0001)$, Pam3CSK4 (23\% and $32 \%$ increases, both $\mathrm{p}<0.0001)$ and $\mathrm{FSL}-1$ stimulation $(17 \%$ and $20 \%$ increases, both p $<0.01)$. Similarly, CD4+ T cell CD38 expression was elevated following incubation with both a low and high dose of LPS (69\% and 73\% increases, both p<0.0001), Pam3CSK4 (58\% and 73\% increases, both $p<0.0001)$ and FSL-1 (50\% and 53\% increases, both $p<0.0001)$ (Table 5.2).

The raw results (Figure 5.7A and Figure 5.7C) and relative results (Figure 5.7B and Figure 5.7D) for CD38 expression with the addition of platelets are given below. Platelet co-culture modestly increased $\mathrm{CD} 8+\mathrm{T}$ cell $\mathrm{CD} 38$ expression, with a relative increase of $5 \%$ in response to low-dose Pam3CSK4 $(p<0.05)$ and of $8 \%$ in response to high-dose FSL-1 $(p<0.05$, also in Table 5.3). Platelets did not change CD8+ T cells expression of CD38 under any other condition tested. Under all conditions tested, no change in CD4+ T cell expression of CD38 was seen with platelet co-culture. 

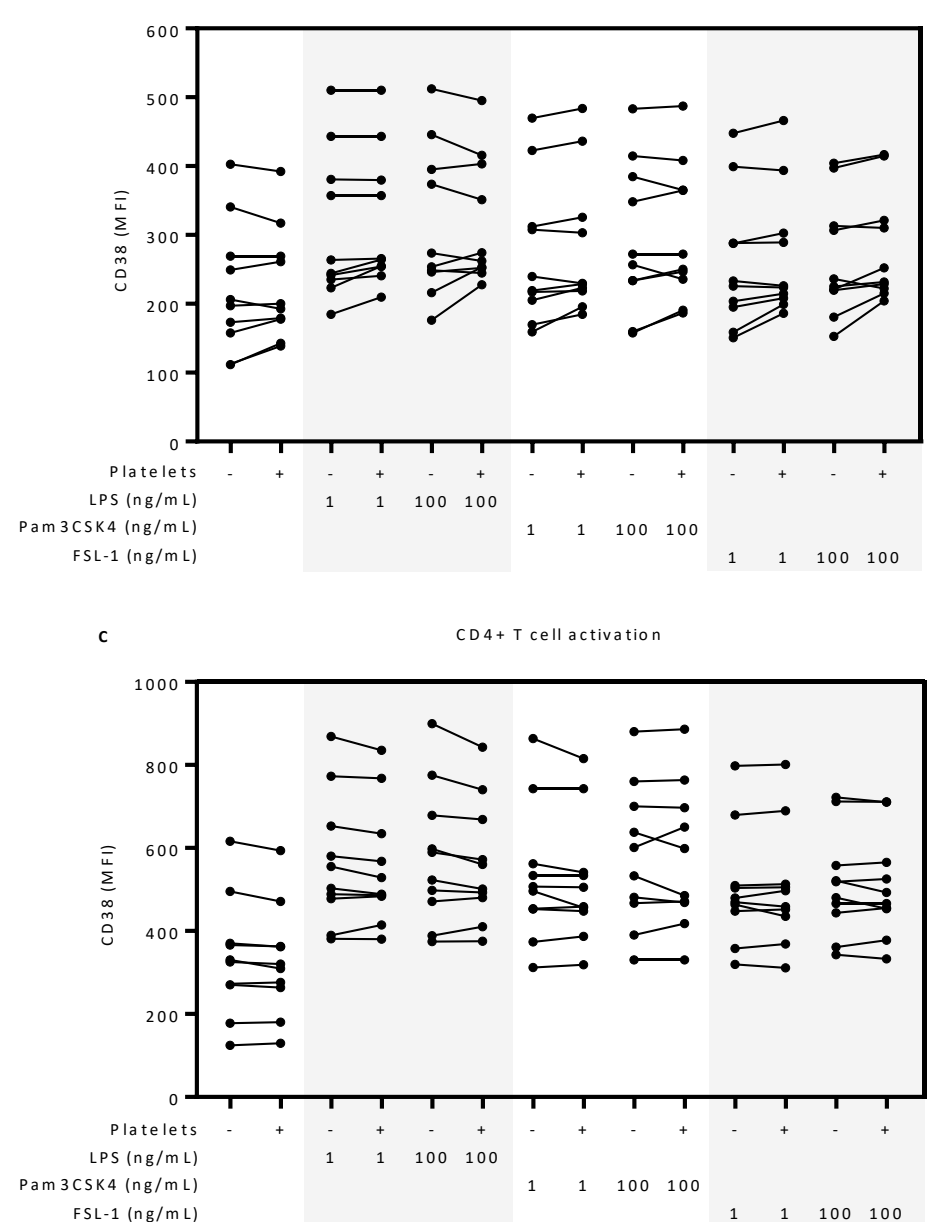
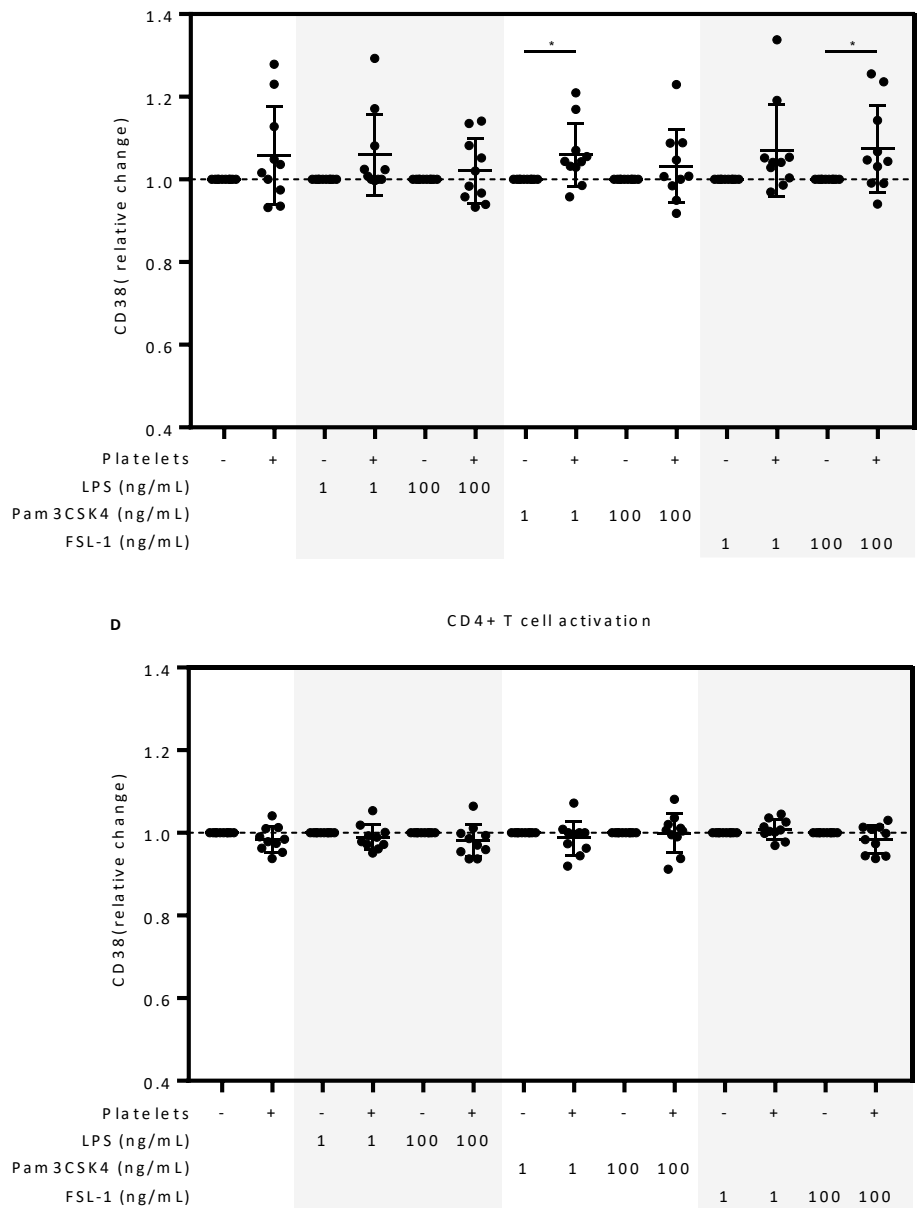

Figure 5.7 Platelets modulate expression of $\mathrm{CD} 8+$, but not $\mathrm{CD} 4+, \mathrm{T}$ cell expression of CD38 following TLR stimulation.

Raw CD8+ T cell (A) and CD4+ T cell (C) CD38 expression was measured (MFI) with (+) and without (-) platelets in response to LPS, Pam3CSK4 and FSL-1. CD38 expression was calculated as a relative change for $C D 8+T$ cells $(B)$ and $C D 4+T$ cells $(D)$. Differences between these relative change measurements were examined using paired t-tests. ${ }^{*} p<0.05$ 


\subsubsection{Platelet co-culture reduces elastase secretion following low-dose TLR agonism.}

Elastase secretion in response to TLR agonism with and without platelets was assessed in the granulocyte culture supernatant. In this study, approximately $89-95 \%$ of the granulocytes in culture were identified as CD16+ neutrophils. We suggest that, in granulocyte cultures, neutrophils are the predominant source of elastase secretion; therefore, elastase secretion can act as a marker of neutrophil activation. In comparison to baseline (- platelets) unstimulated cultures ( $32 \mathrm{ng} / \mathrm{mL}$ ), elastase secretion increased in response to both low-dose and high-dose LPS (58 and $59 \mathrm{ng} / \mathrm{mL}$, respectively, both $\mathrm{p}<0.05$ ), Pam3CSK4 (66 and $63 \mathrm{ng} / \mathrm{mL}$, both $p<0.01$ ) and FSL1 (71 and $65 \mathrm{ng} / \mathrm{mL}$, both $\mathrm{p}<0.01$ ). The increase in elastase secretion was relatively uniform across TLR stimuli. Platelets attenuated elastase secretion in response to low doses of each TLR agonist (presented in Table 5.4 and in both Figure 5.8A and Figure 5.8B). In the presence of platelets, the increase in elastase secretion was attenuated by $17 \%$ in response to low-dose LPS, attenuated by $21 \%$ in response to low-dose Pam3CSK4, and attenuated by $17 \%$ in response to low-dose FSL-1 (all $p<0.01$ ). 
Table 5.4 Relative change in elastase secretion $(\mathrm{ng} / \mathrm{mL}$ ) in granulocyte-platelet co-culture (+ platelets) and following TLR stimulation.

\begin{tabular}{|l|l|l|}
\cline { 3 - 3 } \multicolumn{2}{|c|}{} & \multicolumn{1}{c|}{ + platelets $^{\#}$} \\
\hline Agonist & $\mathrm{ng} / \mathrm{mL}$ & Elastase secretion $(\mathrm{ng} / \mathrm{mL})$ \\
\hline US & -- & $0.85(0.25)$ \\
& 1 & $0.83(0.14)^{* *}$ \\
Pam3CSK4 & 1 & $0.89(0.22)$ \\
& 100 & $0.79(0.11)^{* * *}$ \\
FSL-1 & 100 & $0.92(0.27)$ \\
& 1 & $0.83(0.14)^{* *}$ \\
& 100 & $0.89(0.20)$ \\
\hline
\end{tabular}

\#all leukocyte only measurements were normalized to 1 , and all co-culture (+ platelets) measurements were compared to this normalized response. Differences between these measurements were examined by paired t-tests. ${ }^{* *} p<0.01$, $* * * \mathrm{p}<0.001$. 
Elastase secretion

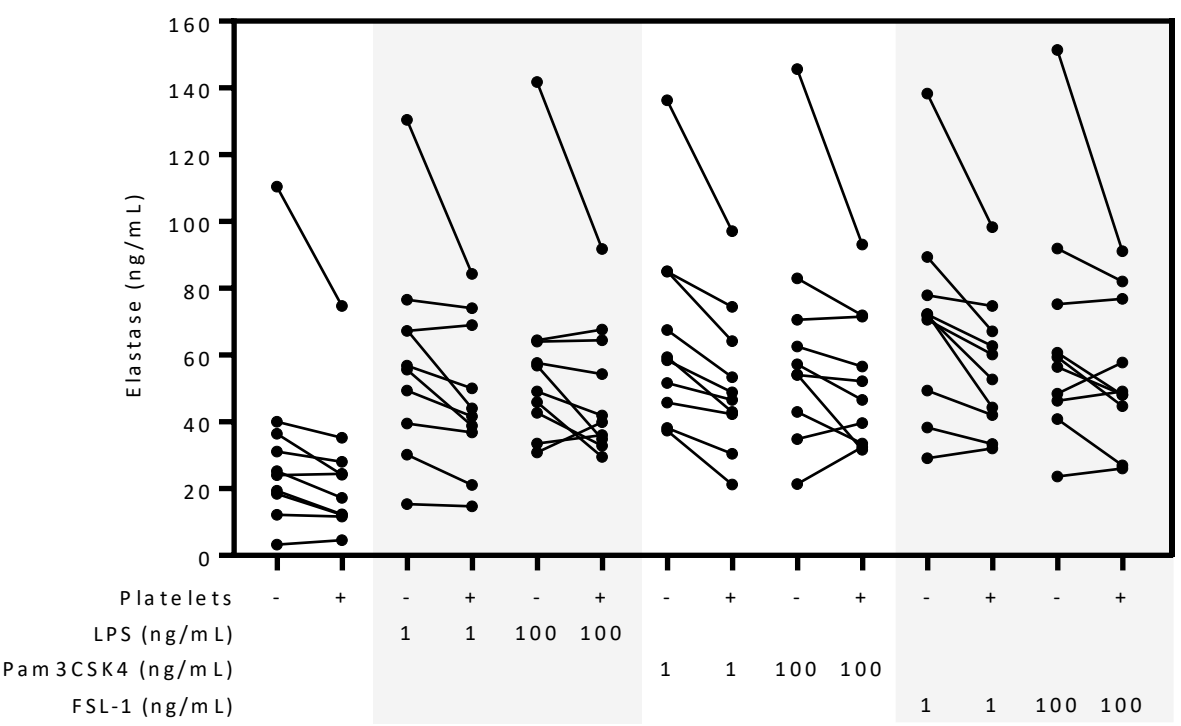

B

Elastase secretion

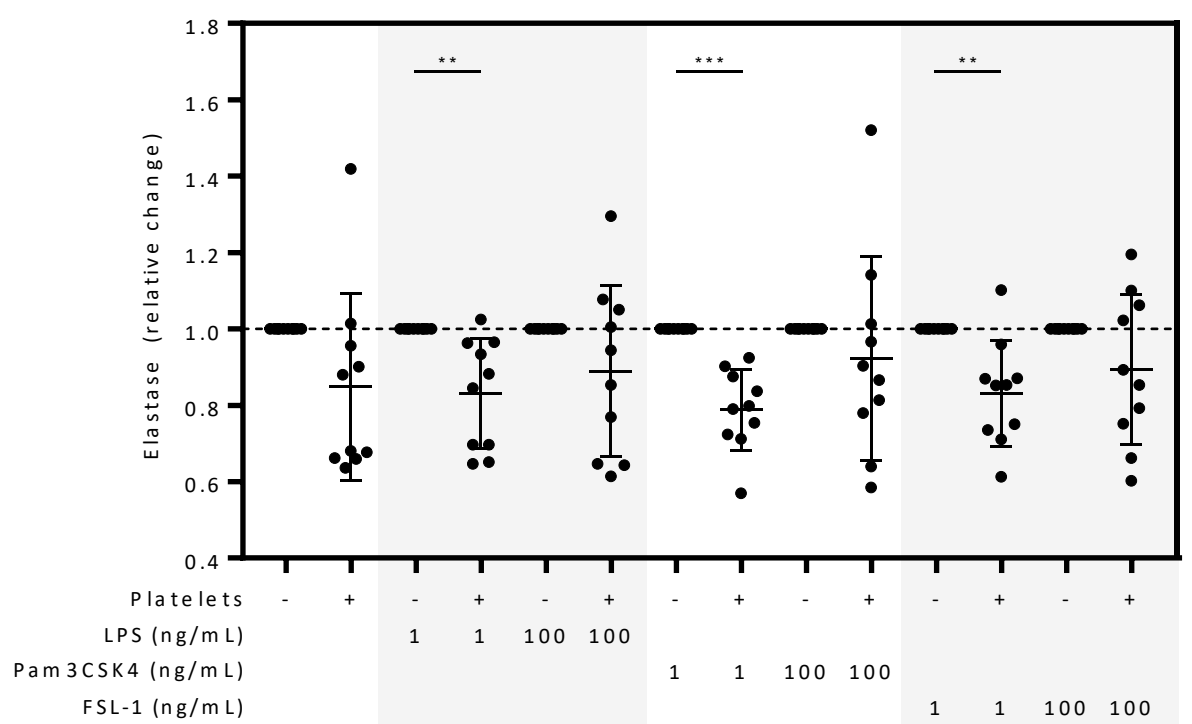

Figure 5.8 Platelets attenuated granulocyte elastase secretion in response to low-dose TLR agonism.

Elastase secretion was measured in granulocyte only cultures (- platelets) and granulocyte + platelet co-cultures (+ platelets) with no stimulation and in response to each TLR agonist (A). Elastase secretion was calculated as a relative change (B). Differences between these relative change measurements were examined using paired t-tests. ${ }^{* *} p<0.01$, $* * * p<0.001$. 


\subsubsection{Platelets differentially modulate IL-6 production in response to LPS and FSL-1.}

The production of a range of cytokines and chemokines was assessed in the PBMC \pm platelet culture supernatant, including IL-6. At baseline (- platelets) and in comparison to unstimulated PBMCs $(20.6 \mathrm{pg} / \mathrm{mL}), \mathrm{IL}-6$ production increased in response to low-dose and high-dose LPS (2851.3 and $3942.6 \mathrm{pg} / \mathrm{mL}$, respectively, both $\mathrm{p}<0.01)$ and high-dose Pam3CSK4 $(1633.0 \mathrm{pg} / \mathrm{mL}, \mathrm{p}<0.05)$. IL-6 production was numerically higher following stimulation with low-dose Pam3CSK4 (310.9 $\mathrm{pg} / \mathrm{mL}$ ) and both doses of FSL-1 (214.8 and $651.0 \mathrm{pg} / \mathrm{mL}$ ). As with monocyte HLA-DR expression, large inter-individual variability was noted for IL-6 measurements, and in all cytokine measurements, which may explain why these measurements did not achieve significance.

The raw IL-6 measurements are shown in Figure 5.9A, and the relative changes with platelet coculture are shown in Figure 5.9B and in Table 5.5. The increase in IL-6 production in response to both doses of LPS was attenuated by $14 \%$ in the presence of platelets (both $p<0.05$ ). The increase in IL-6 production in response to high-dose FSL-1 was further significantly increased by $37 \%$ with platelet co-culture (both $\mathrm{p}<0.05$ ). In response to low-dose FSL-1, IL-6 production was increased by $53 \%$, although this was not detected as significant. No change in IL-6 production was seen in response to Pam3CSK4 when platelets were added. 
Table 5.5 Relative change in PBMC cytokine and chemokine production in PBMC-platelet co-culture (+ platelets) and following TLR stimulation.

\begin{tabular}{|c|c|c|c|c|c|}
\hline \multirow[b]{2}{*}{ Agonist } & \multirow[b]{2}{*}{$\mathrm{ng} / \mathrm{mL}$} & \multicolumn{4}{|c|}{+ platelets ${ }^{\#}$} \\
\hline & & IL-6 & TNF- $\alpha$ & IL-10 & MIP-1 $\beta$ \\
\hline US & -- & $0.94(0.30)$ & $0.95(0.38)$ & $1.04(0.26)$ & $0.88(0.30)$ \\
\hline \multirow[t]{2}{*}{ LPS } & 1 & $0.86(0.14)^{* *}$ & $0.81(0.09)^{* * * *}$ & $1.02(0.19)$ & $0.88(0.15)^{*}$ \\
\hline & 100 & $0.86(0.16)^{*}$ & $0.85(0.13)^{* *}$ & $1.19(0.17)^{* *}$ & $1.01(0.32)$ \\
\hline \multirow[t]{2}{*}{ Pam3CSK4 } & 1 & $1.25(0.66)$ & $0.85(0.26)$ & $1.08(0.11)$ & $1.06(0.27)$ \\
\hline & 100 & $1.24(0.36)$ & $0.90(0.15)$ & $1.19(0.33)$ & $1.09(0.20)$ \\
\hline \multirow[t]{2}{*}{ FSL-1 } & 1 & $1.53(0.81)$ & $0.84(0.09)^{* * *}$ & $1.05(0.07)^{*}$ & $1.23(0.27)^{*}$ \\
\hline & 100 & $1.38(0.51)^{*}$ & $0.81(0.16)^{* *}$ & $1.14(0.15)^{*}$ & $1.15(0.20)^{*}$ \\
\hline
\end{tabular}

\#all leukocyte only measurements were normalized to 1, and all co-culture (+ platelets) measurements were compared to this normalized response. Differences between these measurements were examined by paired t-tests. ${ }^{*} p<0.05,{ }^{* *} p<0.01$, $* * * p<0.001, * * * * p<0.0001$. 
IL-6 production

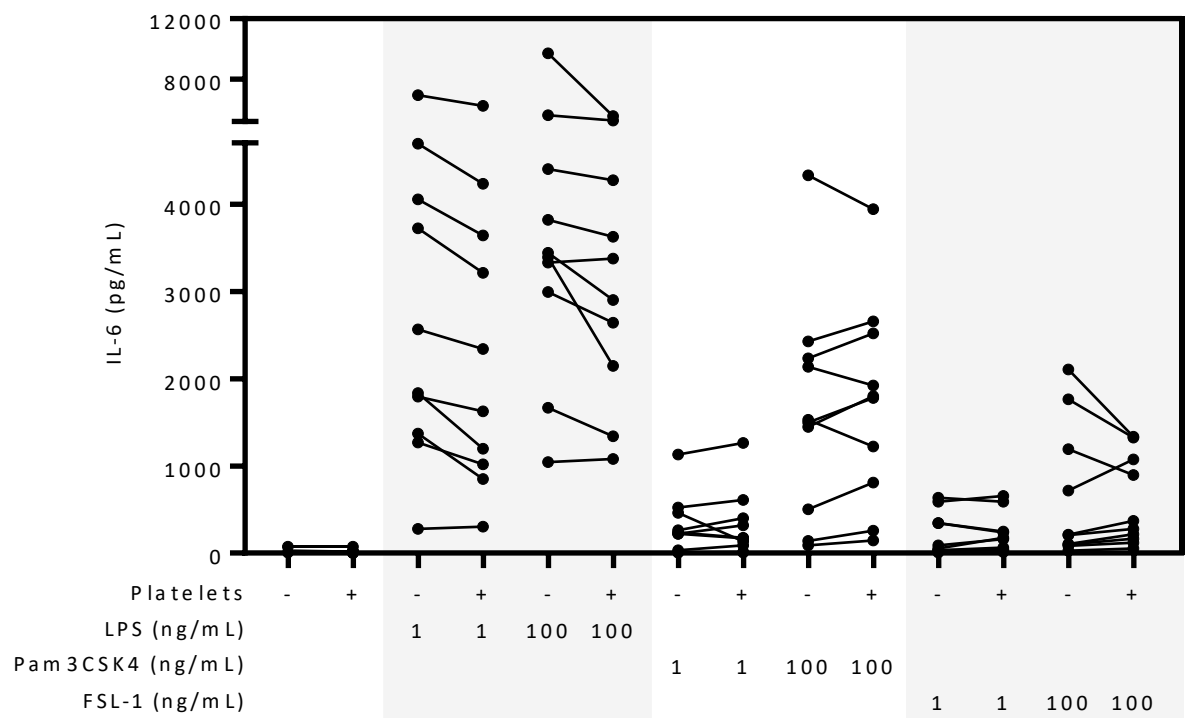

B

IL-6 production

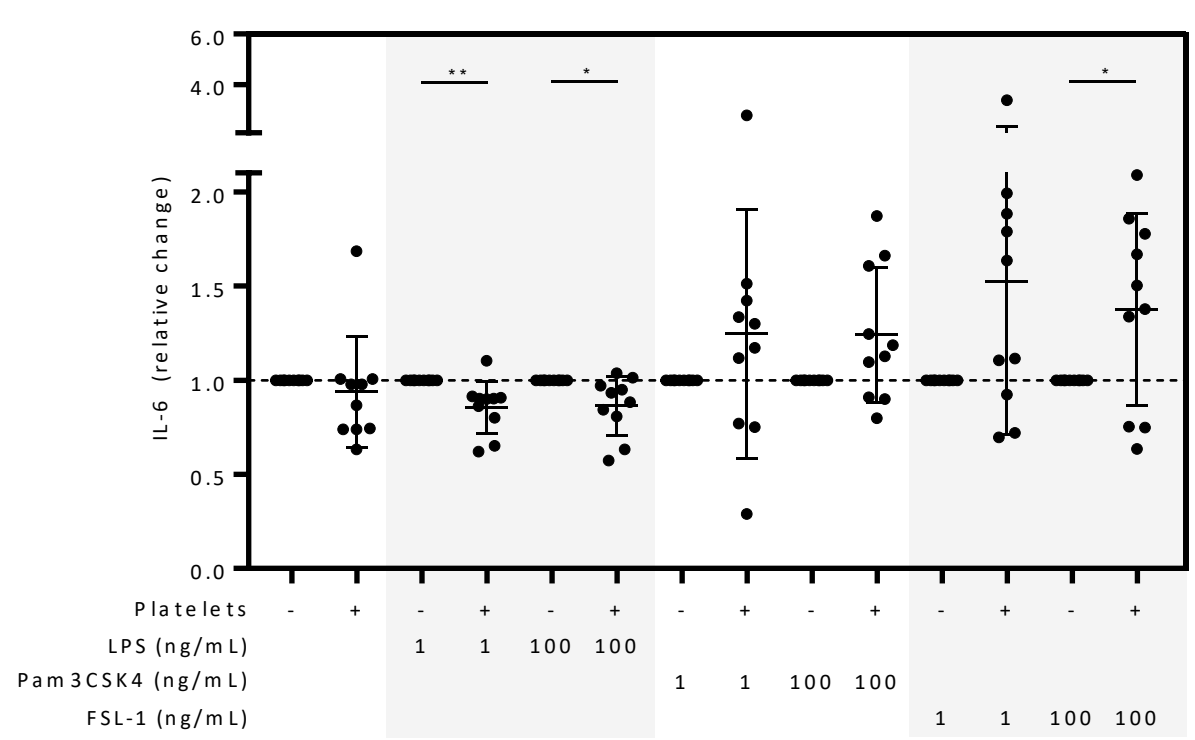

Figure 5.9 Distinct patterns of IL-6 production were seen following TLR stimulation and platelet co-culture.

IL-6 was measured in a multiplex assay in PBMC only cultures (- platelets) and PBMC + platelet co-cultures (+ platelets) with no stimulation and in response to each agonist (A). IL-6 production was calculated as a relative change (+ platelets/- platelets) (B). Differences between these measurements were examined using paired t-tests. ${ }^{*} p<0.05$, $* * p<0.01$. 


\subsubsection{Platelets reduce TNF- $\alpha$ production in response to LPS and FSL-1.}

Relative to baseline (- platelets) unstimulated PBMCs $(214.6 \mathrm{pg} / \mathrm{mL})$, elevated TNF- $\alpha$ levels were seen following stimulation with low-dose and high-dose LPS (3140.2 and 4430.4 pg/mL, both $\mathrm{p}<0.05)$ and high-dose Pam3CSK4 stimulation $(1289.2 \mathrm{pg} / \mathrm{mL}, \mathrm{p}<0.05)$ and levels were numerically higher following stimulation with low-dose Pam3CSK4 (548.3 pg/mL) and FSL-1 (408.0 and 780.4 $\mathrm{pg} / \mathrm{mL})$.

In the presence of platelets, the increase in TNF- $\alpha$ production was attenuated by $19 \%$ following stimulation with low-dose LPS and by $15 \%$ with high-dose LPS (both $p<0.05$ ). These results are presented in Figure 5.10 and Table 5.5. The increase in TNF- $\alpha$ production in response to FSL-1 production was also attenuated by platelets, with a $16 \%$ reduction in response to low-dose FSL-1 and a $19 \%$ reduction in response to high-dose FSL-1 (both $p<0.01$ ). 
A

TNF- $\alpha$ production
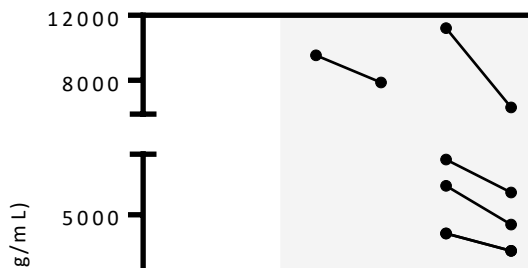

an

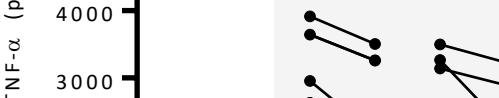

$\stackrel{z}{\longrightarrow}$

TNF- $\alpha$ production

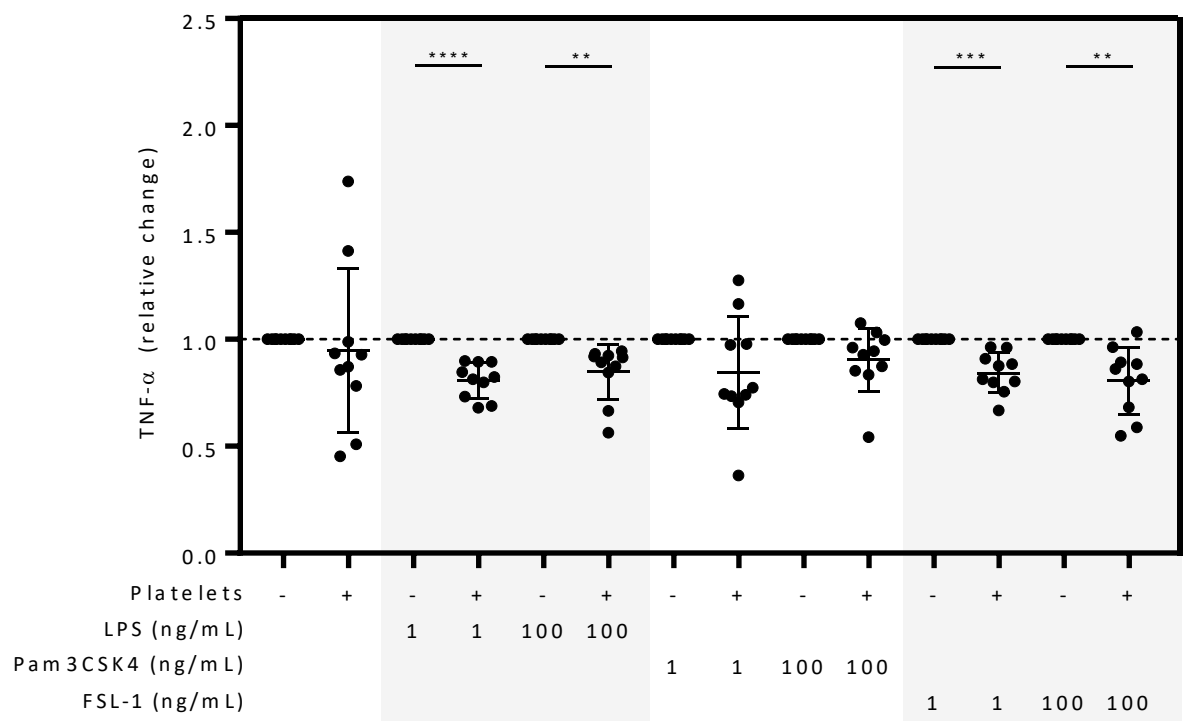

Figure 5.10 TNF- $\alpha$ levels were attenuated with platelet co-culture following stimulation with LPS and FSL-1.

TNF- $\alpha$ was measured in PBMC only cultures (- platelets) and co-cultures (+ platelets) with no stimulation and in response to each agonist (A). TNF- $\alpha$ production was calculated as a relative change (B). Each co-culture measurement was presented as relative change. Differences between these measurements were examined using paired t-tests. ${ }^{* *} p<0.01,{ }^{* * *} p<0.001,{ }^{* * * *} p<0.0001$. 


\subsubsection{Platelets increase IL-10 production by PBMCs in response to LPS and FSL-1.}

Compared to baseline unstimulated cultures $(58.4 \mathrm{pg} / \mathrm{mL}), \mathrm{IL}-10$ levels were raised in response to low and high doses of LPS (442.4 and $442.6 \mathrm{pg} / \mathrm{mL}$, both $\mathrm{p}<0.01$ ), Pam3CSK4 (90.5 and 200.2 $\mathrm{pg} / \mathrm{mL}$, both $\mathrm{p}<0.05)$ and high-dose FSL-1 $(110.8 \mathrm{pg} / \mathrm{mL}, \mathrm{p}<0.05)$. IL-10 production was marginally numerically higher in response to low-dose FSL-1 $(78.2 \mathrm{pg} / \mathrm{mL})$. The increase in IL-10 production in response to high-dose LPS was elevated by $19 \%$. Also, in response to FSL-1 was elevated by $5 \%$ (low-dose) and 14\% (high-dose) (all p<0.05, Table 5.5, Figure 5.11). 


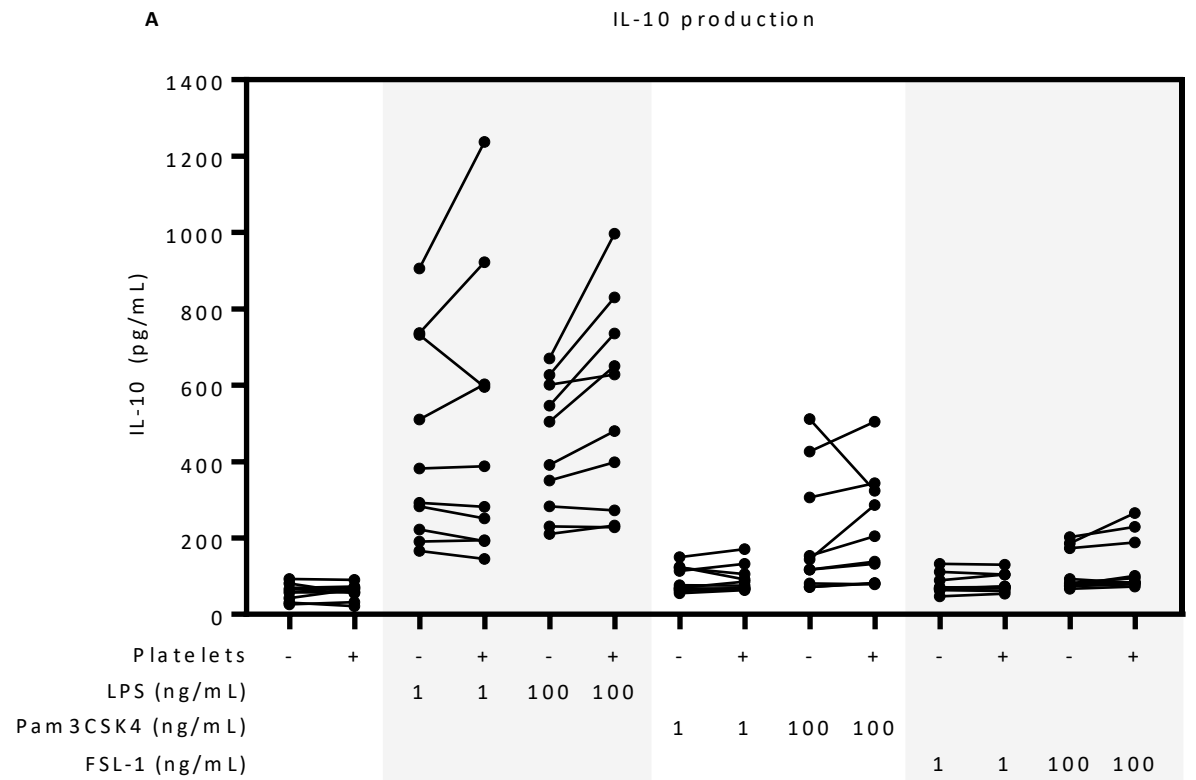

IL-10 production

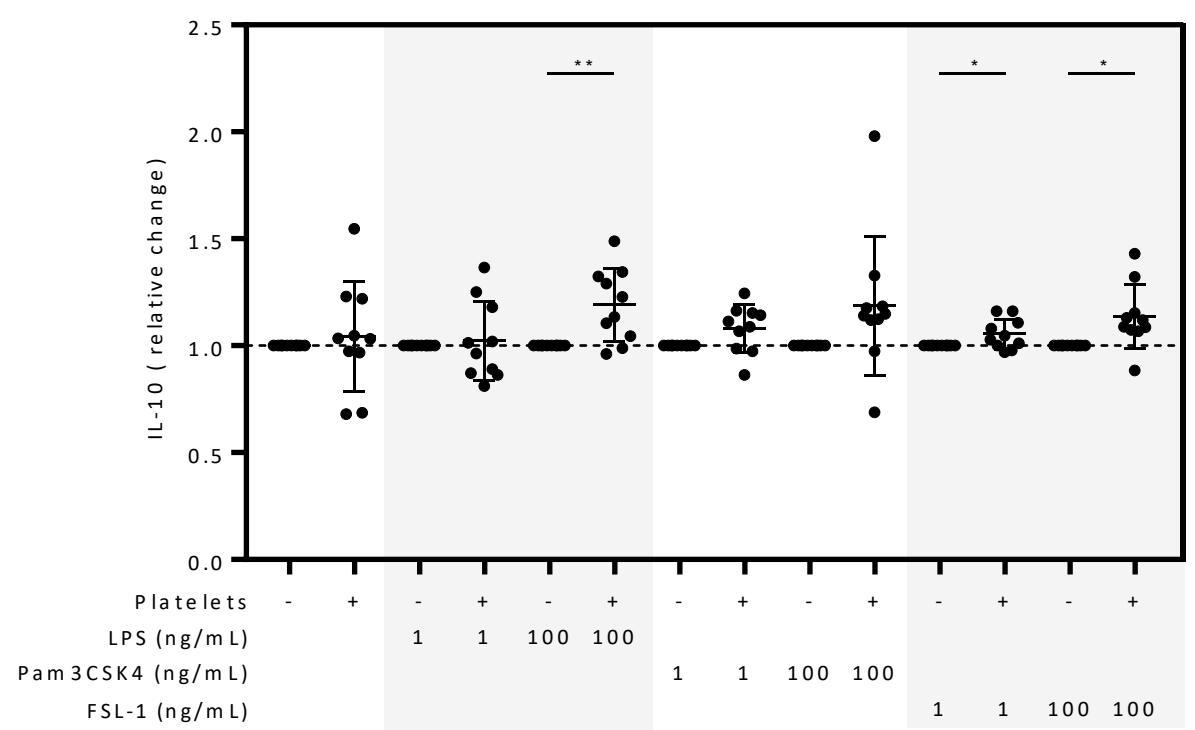

Figure 5.11 IL-10 production was increased following stimulation with high-dose LPS and with FSL-1 and platelet coculture.

IL-10 production was measured in PBMC-only cultures and in co-cultures with no stimulation in response to each agonist (A). IL-10 production was presented as a relative change (B). Differences between these measurements were examined using paired t-tests. ${ }^{*} p<0.05,{ }^{*} p<0.01$. 


\subsubsection{Platelets differentially modulate MIP-16 production in response to some TLR agonists.}

In comparison to unstimulated PBMCs $(3.1 \mathrm{ng} / \mathrm{mL})$, baseline MIP-1 $\beta$ levels were elevated following low-dose and high-dose LPS stimulation (61.5 and $100.8 \mathrm{ng} / \mathrm{mL}$, both $\mathrm{p}<0.05$ ) and Pam3CSK4 stimulation (9.2 and $23.4 \mathrm{ng} / \mathrm{mL}$, both $\mathrm{p}<0.05$ ) and were numerically higher following $\mathrm{FSL}-1$ stimulation (8.4 and $15.2 \mathrm{ng} / \mathrm{mL}$ ). The increase in MIP-1 $\beta$ production following low-dose LPS was attenuated by $12 \%$ in platelet co-cultures $(p<0.05)$ (Table 5.5, Figure 5.12$)$. Conversely, in the presence of platelets, the MIP-1 $\beta$ response was further elevated by $23 \%$ (low-dose) and 15\% (highdose) in response to FSL-1 (both $p<0.05)$. 
A

MIP-1 $\beta$ production

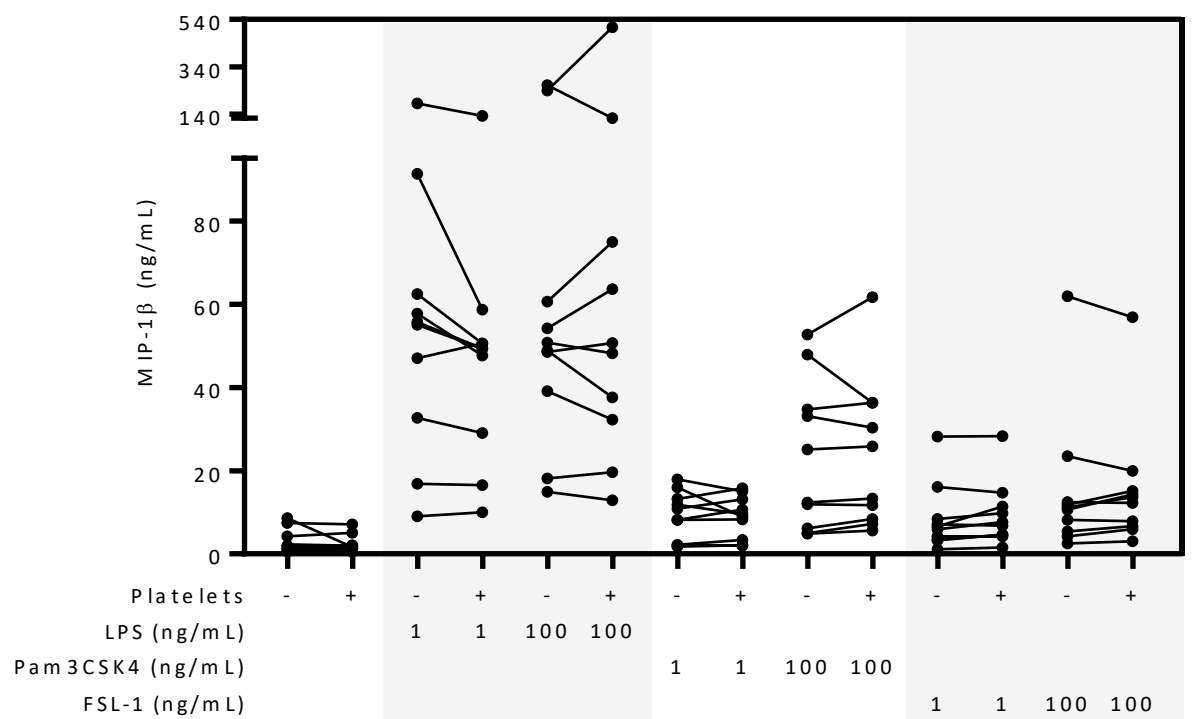

B

MIP-1 $\beta$ production

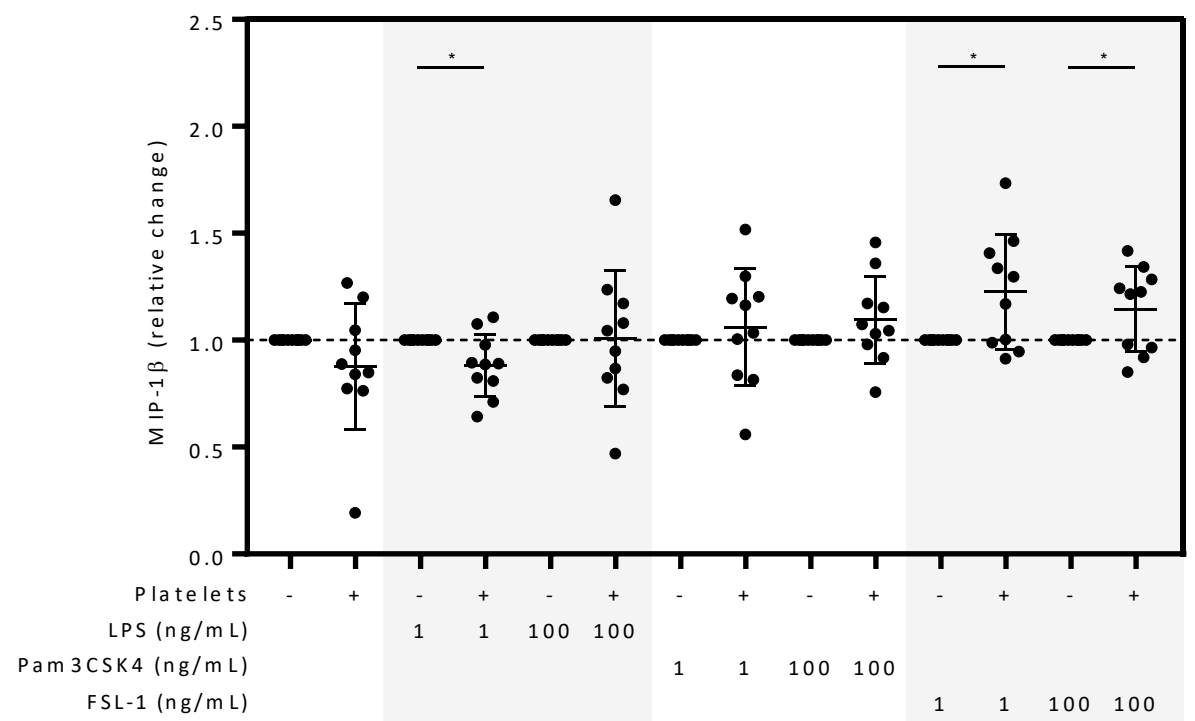

Figure 5.12 Platelets modulate MIP-1 $\beta$ production were seen following stimulation with low-dose LPS and with FSL-1.

MIP-1 $\beta$ production was measured in PBMC-only cultures and co-cultures with no stimulation and in response to each agonist (A). MIP-1 $\beta$ production was presented as a relative change (B). Differences between these measurements were examined using paired t-tests. ${ }^{*} p<0.05,{ }^{* *} p<0.01$. 


\subsection{Discussion.}

In this study, we show that platelets differentially modulated leukocyte responses to TLR stimulation. Platelets attenuated neutrophil expression of CD66b in response to stimulation with various doses of Pam3CSK4 (a TLR2/1 agonist), LPS (TLR4) and FSL-1 (TLR2/6), and modestly attenuated monocyte expression of HLA-DR in response to high-dose LPS. Conversely, platelets modestly increased CD8+ T-cell expression of CD38 in response to low-dose Pam3CSK4 and highdose FSL-1, although platelets did not change CD38 expression on CD4+ T-cells across all TLR agonists tested. Granulocyte elastase production, used here as a marker of neutrophil activation, was attenuated in the presence of platelets following stimulation with a low-dose of each TLR agonist used. PBMC cytokine and chemokine production was also assessed and we demonstrate that, in response to LPS, production of IL-6, TNF $\alpha$ and MIP-1 $\beta$ decreased and IL-10 production increased with platelet co-culture. Conversely, in response to FSL-1, the presence of platelets increased IL-6, IL-10 and MIP-1 $\beta$ production, but attenuated TNF- $\alpha$ production by PBMCs. However, platelets did not alter PBMC cytokine production in response to Pam3CSK4. These results are summarized in Table 5.6 below. 
Table 5.6 Summary of the effect of platelets on the markers of leukocyte function assessed in this study.

\begin{tabular}{|c|c|c|c|c|c|c|c|c|c|c|}
\hline \multirow[b]{2}{*}{ Agonist } & \multirow[b]{2}{*}{$\mathrm{ng} / \mathrm{mL}$} & \multicolumn{9}{|c|}{ +platelets } \\
\hline & & $\begin{array}{l}\text { Neutrophil } \\
\text { CD66b }\end{array}$ & $\begin{array}{l}\text { Monocyte } \\
\text { HLA-DR }\end{array}$ & $\begin{array}{l}\text { CD8+ T cell } \\
\text { CD38 }\end{array}$ & $\begin{array}{l}\text { CD4+ T cell } \\
\text { CD38 }\end{array}$ & $\begin{array}{l}\text { Elastase } \\
\text { secretion }\end{array}$ & $\begin{array}{l}\text { PBMC IL-6 } \\
\text { production }\end{array}$ & $\begin{array}{l}\text { PBMC TNF- } \alpha \\
\text { production }\end{array}$ & $\begin{array}{l}\text { PBMC IL-10 } \\
\text { production }\end{array}$ & $\begin{array}{l}\text { PBMC MIP-1 } \\
\text { production }\end{array}$ \\
\hline US & -- & & & & & & & & & \\
\hline \multirow[t]{2}{*}{ LPS } & 1 & $\downarrow$ & $\downarrow$ & & & $\downarrow$ & $\downarrow$ & $\downarrow$ & & $\downarrow$ \\
\hline & 100 & & & & & & $\downarrow$ & $\downarrow$ & $\uparrow$ & \\
\hline \multirow[t]{2}{*}{ Pam3CSK4 } & 1 & & & $\uparrow$ & & $\downarrow$ & & & & \\
\hline & 100 & $\downarrow$ & & & & & & & & \\
\hline \multirow[t]{2}{*}{ FSL-1 } & 1 & $\downarrow$ & & & & $\downarrow$ & & $\downarrow$ & $\uparrow$ & $\uparrow$ \\
\hline & 100 & $\downarrow$ & & $\uparrow$ & & & $\uparrow$ & $\downarrow$ & $\uparrow$ & $\uparrow$ \\
\hline
\end{tabular}

Orange shaded/ $\uparrow$ Significant increase, blue shaded/ $\downarrow$ significant decrease in relative change. 
Overall, these results indicate that platelets can modestly regulate leukocyte function in both a cell-specific and TLR agonist-specific manner. The results from this study also challenge the notion that platelets act solely in a pro-inflammatory manner [21,305]. Rather, the involvement of platelets in inflammation is likely to be complex, context-dependent and may provide both proand anti-inflammatory cues. This study adds to the growing body of evidence which suggests that platelets can actively participate in limiting leukocyte functions, a mechanism which is postulated to prevent uncontrolled inflammation that would otherwise cause host damage (for a recent review, see[22]). In agreement with this thinking, others have shown that platelets can reduce a number of leukocyte-mediated pro-inflammatory processes [23, 24, 27, 306], and these effects are likely to redefine the role of platelets in a range of clinical settings including sepsis, systemic inflammation and wound healing, to name a few processes. We add to this by suggesting that some, but not other, leukocyte subsets are more sensitive to the presence of platelets and these particular platelet-leukocyte interactions dampened the pro-inflammatory response to TLR stimulation.

We observed a significant increase in neutrophil expression of CD66b at baseline (in the absence of platelets) in response to all three TLR agonists used in this study. The addition of platelets modestly attenuated this increase in CD66b expression at particular doses of each TLR agonist, and this platelet effect was more evident and consistent in response to stimulation with FSL-1. In addition, a reduction in elastase production in granulocyte cultures was seen in the presence of platelets in response to low-dose TLR stimulation. Previous studies have also shown that platelets can suppress neutrophil function. Corken et al. have shown that the loss of platelet GP Ib-IX enhances neutrophil expression of MAC-1 (CD11b) in a mouse model of sepsis [18]. This highlights a platelet-dependent pathway that modulates neutrophil activation in the context of sepsis, which is a potent source of TLR-triggering PAMPs and DAMPs. Hurley et al. demonstrated that, in response to Streptococcus pyogenes M1 protein (another TLR-triggering molecule), plateletneutrophil complexes formed in cell culture and, interestingly, these complexes exhibited attenuated chemotaxis and bacterial killing [307]. It is likely that, in these models of sepsis, platelets and neutrophils are exposed to a multitude of stimulating molecules, including a mixture 
of TLR agonists. We are the first to demonstrate that platelets can also dampen neutrophil activation in response to very specific, singular TLR stimulation. Others have shown that platelets can limit neutrophil function in response to non-TLR agonists. For example, platelets were shown to reduce total elastase levels [308], also demonstrated by this study, and reduce production of ROS $[28,309]$ by neutrophils stimulated with the calcium ionophore A23187 and the chemotactic protein $\mathrm{fMLP}$.

It is interesting to note that, for each TLR agonist used in this study, elastase production was relatively similar between the two doses used, but platelets significantly attenuated elastase production only in response to low-dose TLR stimulation. This would suggest that low-dose TLR stimulation is sufficient to induce a strong release of elastase by neutrophils, but it may be that the microenvironment induced by high-dose TLR stimulation limits the ability of platelets to have the regulatory effect that is seen at low-dose TLR stimulation.

We are the first to demonstrate that platelets can dampen neutrophil CD66b expression and elastase secretion in response to TLR agonism although, from the measurement of only two markers in this study, we do not know whether platelets dampen other aspects of neutrophil function in response to TLR agonism. Expanding the number of markers examined such as phagocytosis, which is crucial for effective neutrophil-mediated host defence, may give greater insight into whether platelets provide a consistent dampening cue or whether platelets differentially regulate neutrophil function.

We also observed that platelets modestly attenuated monocyte activation, as evidenced by HLADR expression, in response to low-dose LPS. This modest attenuation was not seen following stimulation with either high-dose LPS, Pam3CSK4 or FSL-1. Stimulation with these agonists resulted in marked increases in HLA-DR expression compared to unstimulated cultures at baseline, but this was not significantly altered in the presence of platelets. It has previously been shown 
that, in platelet-PBMC co-culture, activated platelets slightly but non-significantly attenuated monocyte HLA-DR expression in response to LPS [23]. Similarly, platelet microparticles can reduce HLA-DP DQ DR expression on monocyte-derived dendritic cells, and the authors suggest that monocytes that come into contact with platelet microparticles may be less likely to develop into fully pro-inflammatory dendritic cells [27]. Overall, we suggest that monocyte HLA-DR expression is only moderately influenced by platelets, and this modulation may be secondary to modulation of other monocyte functions by platelets, such as cytokine production.

In this study, platelets modulated cytokine and chemokine production by PBMCs in a complex manner, and this modulation differed between the three TLR agonists tested here. In the context of stimulating PBMCs with TLR agonists, it is likely that the cytokine and chemokine response is driven primarily by monocytes [310] as this leukocyte subset is most responsive and sensitive to direct stimulation with a range of TLR agonists. Platelets did not modulate the PBMC response to Pam3CSK4, the TLR1/2 agonist, for any cytokine or chemokine measured in this study. However, the cytokine/chemokine response to LPS was modulated by platelets. Platelet co-culture lowered IL-6 and TNF- $\alpha$ levels (both traditional pro-inflammatory cytokines), increased IL-10 levels (an antiinflammatory cytokine), and decreased MIP-1ß levels (a pro-inflammatory chemokine) in response to various doses of LPS. These patterns indicate that, in response to LPS, platelets can amplify the production of anti-inflammatory cytokines and attenuate the production of pro-inflammatory cytokines and chemokines by PBMCs. Previous work has described this type of cytokine/chemokine modulation to LPS by platelets. Activated platelets, or activated platelet supernatant, can reduce TNF- $\alpha$ and IL-6 production and induce IL-10 release by both resting [24, 25] and LPS-stimulated [23] human PBMCs as well as LPS-stimulated mouse bone-marrow derived macrophages $[13,311]$. Similarly, platelets that have been exposed to high shear stress can induce IL-10 production in monocyte-derived immature dendritic cells in response to LPS [312]. These collective results suggest that platelets can push PBMCs away from pro-inflammatory, and towards anti-inflammatory, cytokine production in response to LPS. 
The response of PMBCs to FSL-1, a TLR2/6 agonist, in the presence of platelets was more complex. FSL-1-mediated production of IL-6, IL-10 and MIP-1 $\beta$ production was further elevated with platelets, but production of TNF- $\alpha$ was attenuated. This picture, with the up- and down-regulation of traditional pro-inflammatory (IL-6, MIP-1 $\beta$, TNF- $\alpha$ ) and anti-inflammatory (IL-10) cytokines/chemokines, does not clearly demarcate a pro- or anti-inflammatory influence of platelets on the PBMC phenotype in response to FSL-1. It is important to note that FSL-1 did not stimulate PBMC cytokine/chemokine production as effectively as LPS and, when examining relative changes when platelets are added, small absolute changes in production in response to FSL-1 may be represented as large relative changes. However, a small absolute change in cytokine/chemokine production in a low-cytokine/chemokine environment, such as following FSL1 stimulation, may still be physiologically important. These results suggest that platelets have a multifaceted role in balancing pro- and anti-inflammatory responses, which is agonist-specific and which may concurrently facilitate both types of inflammatory responses. To the best of our knowledge, modulation of PBMC cytokine production by platelets in response to FSL-1 has not been previously described.

We observed relatively similar baseline expression levels of monocyte HLA-DR in response to all TLR agonists used in this study, although PBMC cytokine and chemokine production (predominantly driven by monocytes) was differentially and dose-dependently upregulated by TLR stimulation. It may be that lower concentrations $(<1 \mathrm{ng} / \mathrm{mL})$ of these agonists may induce a dosedependent increase in HLA-DR expression that we were unable to see with the doses ( $\geq 1 \mathrm{ng} / \mathrm{mL}$ ) used in this study. We suggest that monocytes are very sensitive to TLR agonism and HLA-DR expression is readily upregulated, perhaps to a maximum level, in response to $\geq 1 \mathrm{ng} / \mathrm{mL}$ of these agonists. The differences between HLA-DR expression and cytokine/chemokine production indicate that these processes are regulated by TLR stimulation in different ways (for example, immediate upregulation in expression versus delayed cytokine/chemokine production) and are, therefore, likely to be affected by the presence of platelets in distinct ways. Indeed, this was observed in our study: platelets had a greater effect on PBMC cytokine/chemokine production than on monocyte HLA-DR expression. 
When examining the baseline (-platelets) results, we demonstrate a robust PBMC cytokine response to LPS and, to a lesser extent, to Pam3CSK4 and FSL-1. Within these cytokine measurements, we also observed substantial inter-individual variation. This is particularly evident when examining cytokine responses to Pam3CSK4 and FSL-1 as, despite stimulation, some of the baseline cytokine measurements were similar to those recorded in unstimulated cultures. This may suggest that there is a population of healthy subjects that exhibit low- or non-responsiveness, in terms of cytokine production, when their PBMCs are exposed to particular TLR agonists. It is difficult to determine whether this is the case with the sample size examined in this study, but it is of interest to determine whether sub-populations of low/non-responders can be identified in a larger cohort of healthy subjects. At an individual level, low/non-responding subjects may have a compromised ability to launch a full and effective cytokine response to particular infective or injurious stimuli.

At a group level, the effect of platelets on various leukocyte measurements was moderate and TLR agonist-specific although we note that, across many of the measurements, the platelet effect was greater in some individuals than in others. It is of interest to determine whether responsiveness to TLR stimulation, as discussed above, is also associated with responsiveness to the addition of platelets. It may be that there are individuals whose leukocytes are more sensitive to adjustments in both agonist as well as cellular stimuli. This question was beyond the scope of the current study, however, but could be achieved in a larger cohort of healthy subjects using cluster analysis.

In PBMC-platelet co-cultures, platelets modestly modulated CD8+ T cell activation, as evidenced by CD38 expression, in response to particular TLR agonists. CD4+ T cell activation was unaltered in the presence of platelets. Modulation of CD8+ T cell expression of CD38 by platelets was TLR agonist- and dose-specific. CD38 is a commonly used T cell activation marker [302] and it has previously been demonstrated that CD38 expression increases on both T cell subsets in response to TLR stimulation, without TCR engagement, in PBMC cultures $[302,303]$. These results are supported by our findings. We demonstrate that, in the absence of TCR stimulation, both T cell 
subsets exhibit increased CD38 expression in response to all three TLR agonists used. The effect of TLR stimulation on T cell CD38 expression may be as a result of bystander activation, in which a cytokine-rich environment can provide cues for $\mathrm{T}$ cell activation in an antigen-independent manner. Bystander activation has been more well-characterized in CD8+ T cells [313], although has also been reported in CD4+ T cells [314], and is postulated to be a mechanism to control early infection in particular T cell subsets [315]. Recent studies have demonstrated that a number of cytokine-producing leukocytes within the PBMC fraction can promote bystander activation including monocytes, natural killer cells and dendritic cells $[316,317]$, and this can occur in response to TLR stimulation [317]. In similar fashion, it may be that platelets moderately affect CD8+ T cell activation indirectly, via interactions with other leukocyte subsets known to induce bystander activation. We show that platelets can modestly enhance TLR-mediated CD8+ T cell activation, and others have also reported that platelets can enhance CD8+ T cell effector functions. For example, platelet depletion in antigen-challenged mice has been shown to reduce the lytic activity [318, 319] and expansion [320] of antigen-activated CD8+ T cells. Collectively, these results suggest that platelets can participate in anti-microbial host defence by modulating CD8+ $T$ cell responses to both classical (TCR engagement) and non-classical (TLR) activation.

In this study, we observed a moderate platelet effect on leukocyte function in a cell-specific and TLR agonist-specific manner. Although moderate, these platelet effects are likely to play an important role in the immune response to infection and sterile injury. We propose that platelets modulate leukocyte function in order to dampen, but not substantially inhibit, inflammation and this notion is supported by others [22]. A rapid and effective pro-inflammatory response that is mounted by both platelets and leukocytes is necessary for pathogenic clearance but this response must also be limited to prevent host damage. This is particularly evident in sepsis, where uncontrolled inflammation is a cause of septic mortality [321], and rigid control of inflammation is required. Aside from this acute inflammatory response, an upset in the balance of pro- and antiinflammatory cytokines can establish persistent, chronic inflammation [322]. This moderate platelet effect does not inhibit important and necessary pro-inflammatory responses but, rather, 
is likely to put a brake on this system to avoid host damage and may mediate, in part, a switch towards anti-inflammatory responses in order to achieve this.

Many of the studies discussed above have examined the effect of platelets on leukocyte function in the context of sepsis, where the inflammatory response is pronounced and known to trigger host damage. These results are likely to be applicable in the context of $\mathrm{MI}$ as well, which is characterized by a large sterile inflammatory response. Cellular damage and necrosis during AMI triggers the release of a number of DAMPs that can activate TLRs, among other inflammatory pathways. It is interesting to postulate how the results of this study may relate to sterile inflammation in AMI. It is possible that platelets may limit certain leukocyte functions during AMI in patients where the resulting sterile inflammatory response is exaggerated or prolonged. Platelets may also act in a site-specific, rather than in a systemic, manner during AMI. For example, the plaque site or the ischaemic myocardium is likely to have an intense inflammatory environment that is not present systemically. The extent to which platelets can modulate leukocyte functions in AMI is an area of highly interesting, but unexplored, research. 


\subsection{Limitations.}

We have deliberately designed this study as a broad "survey" to determine the platelets effect on a range of different responses across a number of different leukocyte subsets. The disadvantage of this design was the inability to wholly characterize the platelet effect on particular leukocyte subset. We believe that it was necessary to broadly characterize these platelet effects in order to determine those effects that warrant further investigation. We did not anticipate the large variability in the raw data for some of the measurements in this study. Combined with the rather moderate relative change in these measurements in the absence or presence of platelets, this limited our ability to detect some differences in the data as statistically significant. However, the cohort was of sufficient size to broadly characterize the platelet effects on leukocyte responses, which was the aim of this study. It was necessary to isolate out different cell fractions from whole blood in order to conduct these experiments, and this may have altered the activation state of these fractions. However, we adapted the experimental protocol to keep the cells in a quiescent state and minimized the number of steps for cell isolation. We did not assess the platelet effect on leukocyte responses to a range of doses, rather only to a low and high dose, of each TLR agonist. However, in order to effectively manage the volume of experimental work, we chose to assess these two doses. We did not assess whether these leukocyte responses were affected by direct platelet interaction or by the indirect release of platelet immunomodulators. However, the literature suggests that the combination of both direct and indirect interactions facilitates the platelet effect, and we focussed on the holistic effect of platelets on leukocyte responses. 


\subsection{Conclusion.}

We show, in this study, that platelets differentially regulate leukocyte responses to TLR stimulation in a TLR agonist-specific and cell-specific manner. Furthermore, platelets were shown to reduce some leukocyte responses in response to TLR stimulation. We suggest that platelets can limit some leukocyte pro-inflammatory processes in response to TLR stimulation, and this platelet effect may play an important role in dampening host damage in response to excessive infectious or injurious inflammation. 
6 Chapter 6. Platelets modulate multiple markers of neutrophil function in response to TLR agonism. 


\subsection{Introduction.}

The role of platelets in inflammation is evolving, with recent evidence suggesting that, aside from mediating important pro-inflammatory processes, platelets can also provide anti-inflammatory cues to dampen leukocyte responses during inflammation (for a recent review, see [22]).

The aim of the study described in Chapter 5 was to broadly assess how platelets modulated certain functions of a number of leukocyte subsets in response to TLR stimulation. We are the first to show that platelets are able to attenuate neutrophil activation in a TLR agonist-specific manner. More specifically, both neutrophil CD66b expression and elastase secretion were attenuated in response to various doses of LPS (a TLR4 agonist), Pam3CSK4 (TLR2/1 agonist), and FSL-1 (TLR2/6 agonist). Due to the broad nature of the previous study, we did not examine the platelet effect on neutrophil function beyond the measurement of CD66b expression and elastase secretion. However, it was of interest to us to further characterize the platelet effect on neutrophil function. In particular, we want to determine whether platelets provide consistent dampening cues (as was seen with CD66b expression and elastase secretion) across a multitude of markers of neutrophil function in response to TLR stimulation.

In this current study, we examined the platelet effect on an expanded number of markers of neutrophil function. Firstly, we wanted to confirm the reproducibility of the platelet effect on CD66b expression and elastase secretion, so these two markers were reassessed in this study. Secondly, we wanted to examine the platelet effect on a wider range of neutrophil activation markers alongside CD66b including cell-surface expression of CD11b and CD62L was assessed here. Expression of CD66b, CD11b and CD62L are well-characterized activation markers [323, 324]. Thirdly, neutrophil secretion of IL-8, an important inflammatory chemoattractant, is a known response to TLR stimulation [254] and was measured in this study. Lastly, phagocytosis is a crucial anti-microbial defence mechanism of neutrophils [325] and was also measured in this study. In 
summary, the platelet effect was assessed on the following markers of neutrophil function: cellsurface CD66b, CD11b, and CD62L expression; elastase and IL-8 secretion; and phagocytic activity. On the basis of our previous results from Chapter 5, we hypothesize that platelets will attenuate various neutrophil functions in a TLR agonist-specific manner. To address this, we aimed:

1. To determine the extent to which platelets can modulate multiple markers of neutrophil function in response to in vitro stimulation with TLR4, TLR2/1, and TLR2/6 agonists in healthy subjects. 


\subsection{Materials and methods.}

\subsubsection{Subject recruitment and blood sampling.}

Blood was collected from 10 consenting healthy subjects ( 5 male, 26 years) as outlined in Chapter 5 (Section 5.2.1).

\subsubsection{Neutrophil isolation.}

Neutrophils were isolated by negative selection from EDTA-anticoagulated whole blood by immunomagnetic depletion of unwanted cells and erythrocyte sedimentation, as per manufacturer's instructions (Miltenyi Biotec, Bergish Gladbach, Germany). Isolated neutrophils were washed and resuspended in cell culture media (see Appendix 2 for recipe) to $1 \times 10^{6}$ neutrophils $/ \mathrm{mL}$, and neutrophils were kept on ice for 60 minutes prior to TLR stimulation.

\subsubsection{Platelet isolation.}

PRP and PPP were isolated from hirudin-anticoagulated whole blood as described in Chapter 5 (Section 5.2.2). A portion of PRP was diluted in PBS to $2.5 \times 10^{8}$ platelets $/ \mathrm{mL}$ and PPP was diluted in PBS in the same ratio as PRP. PRP and PPP were used to assess the platelet effect on neutrophil activation markers. A separate portion of PRP was diluted in PBS with $1 \mu \mathrm{M}$ prostaglandin E1, centrifuged at $100 \times$ g for 12 minutes, and platelets were resuspended in cell culture media to $2.5 \times 10^{8}$ platelets $/ \mathrm{mL}$. Platelets resuspended in cell culture media were used to assess the platelet effect on neutrophil phagocytosis. 


\subsubsection{In vitro TLR stimulation and assessment of neutrophil activation markers.}

Neutrophils were cultured \pm PRP in a ratio of 1:250 neutrophils:platelets. An equal amount of PPP was added to neutrophil-only cultures. Cells were kept at $37^{\circ} \mathrm{C} / 5 \% \mathrm{CO}_{2}$ for 60 minutes prior to TLR stimulation. Neutrophils \pm platelets were either left unstimulated or stimulated with 1 and 100 $\mathrm{ng} / \mathrm{mL}$ of LPS (Enzo Life Sciences), Pam3CSK4 (Tocris Bioscience) and FSL-1 (Santa Cruz Biotechnology). Neutrophils \pm platelets were cultured under these conditions for 4 hours at $37^{\circ} \mathrm{C} / 5 \% \mathrm{CO}_{2}$. After incubation, cells were centrifuged at $400 \times \mathrm{g}$ for 12 minutes, and the cell culture supernatant was collected and stored at $-80^{\circ} \mathrm{C}$. Neutrophils \pm platelets were resuspended in staining buffer and incubated with anti-CD16-BV421 (clone 3G8), anti-CD66b-BB515 (clone G10F5), anti-CD11b/MAC-1-BV510 (clone ICRF44), all sourced from Becton Dickinson, and antiCD62L-APC (DREG-56; BioLegend, San Diego, CA, USA). The panel design for this study is given in Table 6.1. Antibodies were diluted in staining buffer and all samples were stained for 50 minutes at $4^{\circ} \mathrm{C}$. Appropriate isotype controls were run in parallel. Antibody stained neutrophils \pm platelets were fixed with $1 \%$ paraformaldehyde, centrifuged and finally resuspended in staining buffer. All samples were analysed on a FACSCanto II flow cytometer (Becton Dickinson). Flow cytometric data was analysed using FlowJo software (v10.0.7, Tree star). CD16+ neutrophils were identified as outlined in Figure 6.1. The geometric MFI of CD66b, CD11b and CD62L was used to determine CD16+ neutrophil activation and representative plots of each activation marker is given in Figure 6.2. $\mathrm{CD} 66 \mathrm{~b}$ and $\mathrm{CD} 11 \mathrm{~b}$ expression is increased upon neutrophil activation, while CD62L is shed from the surface (expression is attenuated) upon activation. 
Table 6.1 Panel design for assessing leukocyte activation in response to TLR agonism.

\begin{tabular}{|c|l|l|l|}
\hline & $\begin{array}{l}\text { Laser/channel } \\
\text { (fluorophore) }\end{array}$ & $\begin{array}{l}\text { Antibody target } \\
\text { (clone) }\end{array}$ & $\begin{array}{l}\text { Concentration in final staining } \\
\text { volume (final dilution) }\end{array}$ \\
\hline Neutrophil & Blue/Green (BB515) & CD66b (G10F5) & $2.00 \mu \mathrm{g} / \mathrm{mL}(1$ in 50) \\
activation panel & Violet/Blue (BV421) & CD16 (3G8) & $0.25 \mu \mathrm{g} / \mathrm{mL}(1$ in 400) \\
& Violet/Green (BV510) & CD11b (ICRF44) & $4.00 \mu \mathrm{g} / \mathrm{mL} \mathrm{(1} \mathrm{in} \mathrm{50)}$ \\
& Red/Red2 (APC) & CD62L (DREG-56) & $0.25 \mu \mathrm{g} / \mathrm{mL}(1$ in 50) \\
\hline
\end{tabular}

A list of all antibody formats, clones, isotypes and manufacturers can be found in Appendix 1. 

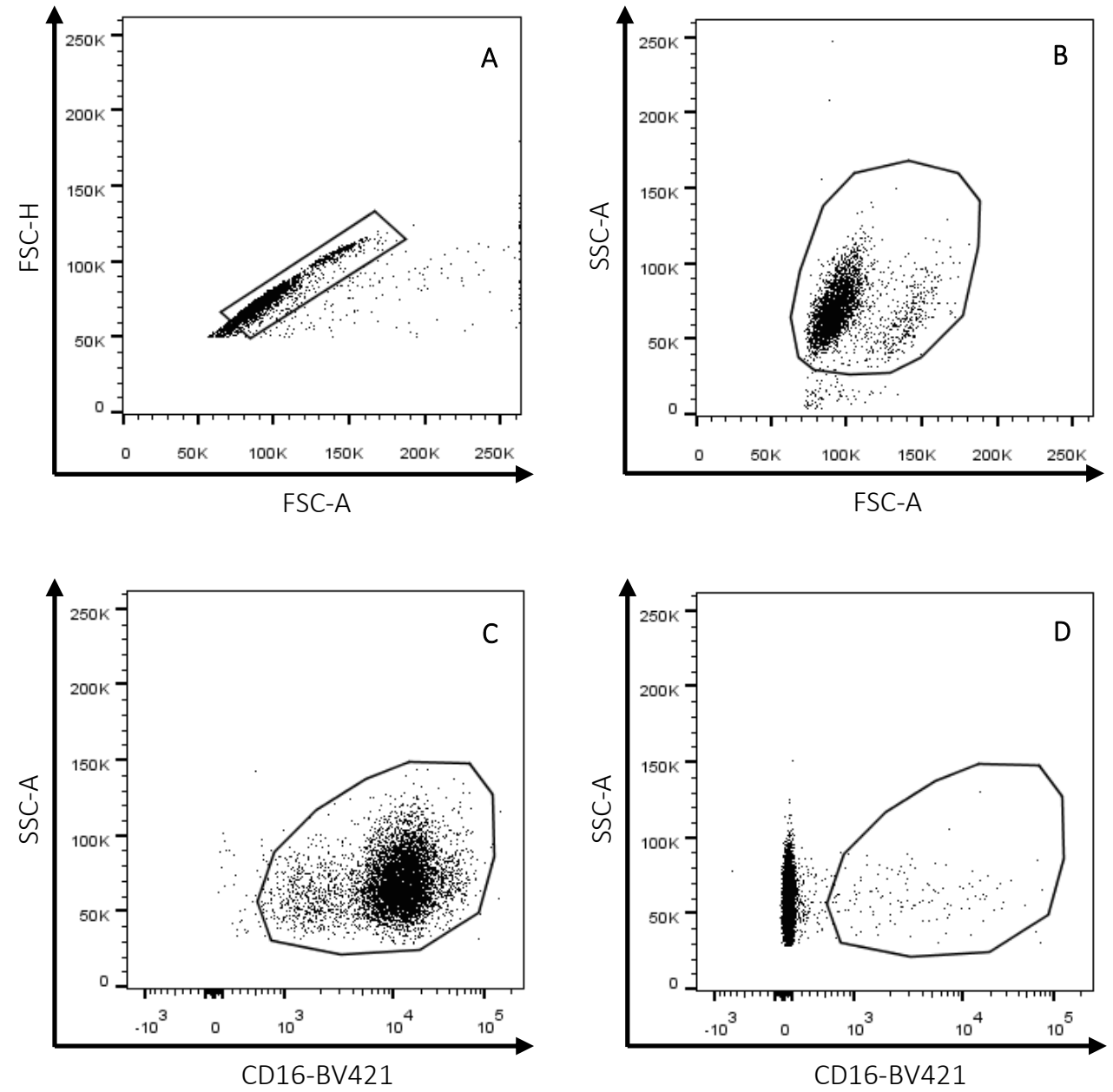

Figure 6.1 CD16+ neutrophils were identified.

Single cells were gated on (A). Neutrophils were identified by their characteristic forward and side scatter profile (B), and by CD16-positivity (C) against the isotype control (D). 

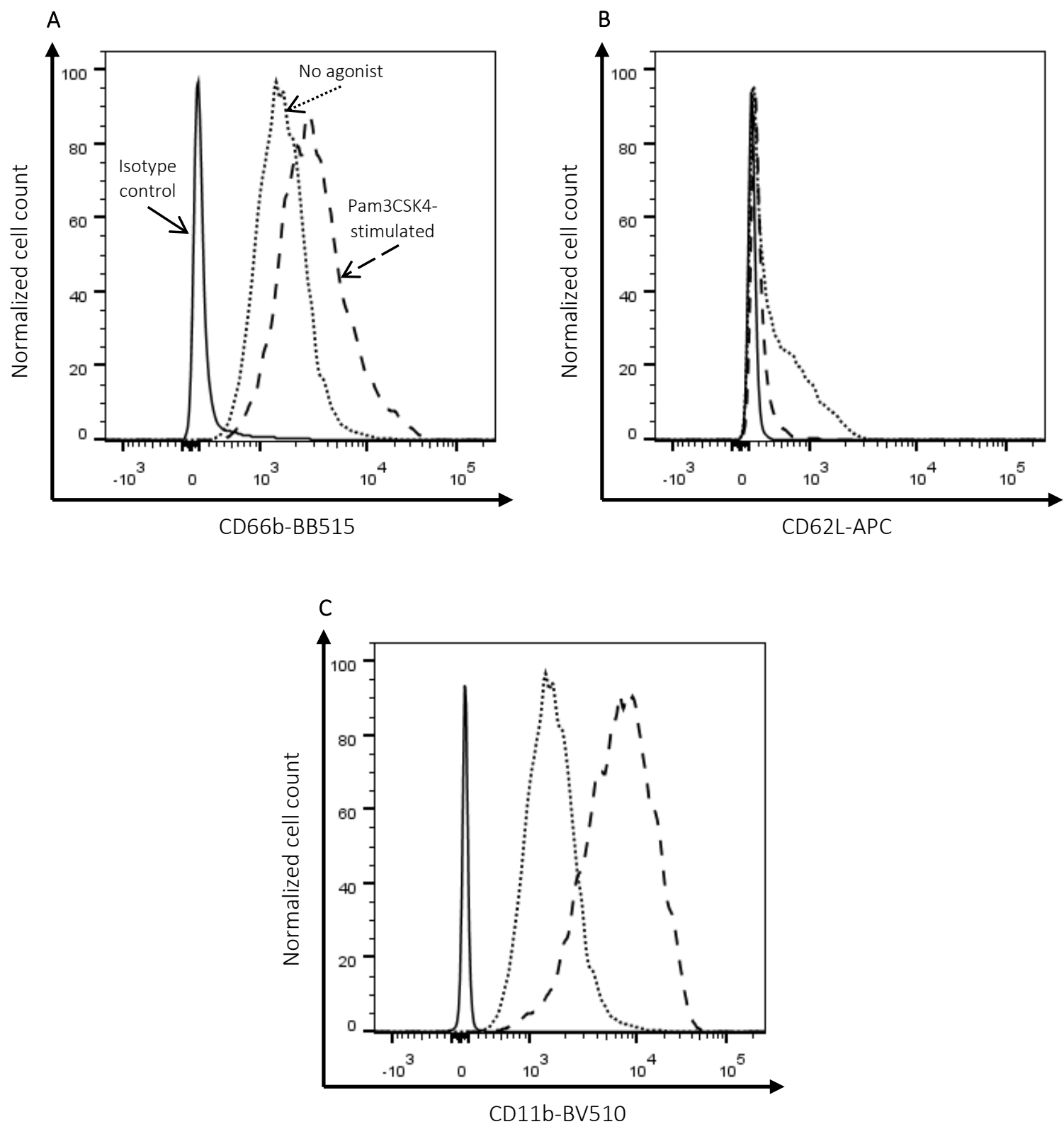

Figure 6.2 Representative plots of neutrophil activation markers.

Representative plots of neutrophil CD66b expression (A), CD62L expression (B) and CD11b expression (C) are shown for unstimulated and Pam3CSK4 (100 ng/mL)-stimulated conditions. CD66b and CD11b expression levels increase following neutrophil stimulation, while CD62L levels decrease (CD62L is shed from the surface). 


\subsubsection{In vitro TLR stimulation and assessment of neutrophil phagocytosis.}

For measurement of neutrophil phagocytic activity, platelets which were resuspended in cell culture media (as described above) were added to neutrophils in a neutrophil:platelet ratio of 1:250. An equal amount of cell culture media was added to neutrophil-only cultures. Neutrophils \pm platelets were incubated with FITC-labelled rabbit IgG-coated latex beads (Cayman Chemicals, Ann Arbor, MI, USA) in a ratio of $1 \mu \mathrm{L}$ of latex beads to every $200 \mu \mathrm{L}$ cell culture media and incubated for 60 minutes at $37^{\circ} \mathrm{C} / 5 \% \mathrm{CO}_{2}$ prior to TLR stimulation. As above, neutrophils \pm platelets were stimulated with 1 and $100 \mathrm{ng} / \mathrm{mL}$ Pam3CSK4, LPS and FSL-1 for 4 hours at $37^{\circ} \mathrm{C} / 5 \% \mathrm{CO}_{2}$. Following stimulation, neutrophils \pm platelets were washed and incubated with Trypan blue diluted in staining buffer to quench fluorescence from cell-surface bound latex beads. All samples were washed, resuspended in 1\% paraformaldehyde, centrifuged and finally resuspended in staining buffer. All samples were then analysed on a FACSCanto II flow cytometer (Becton Dickinson) and FlowJo software (v10.0.7, Tree star). Neutrophils were identified by their characteristic forward and side scatter properties (as shown in Figure 6.1). Phagocytosis was reported as the percentage of the neutrophil population that was FITC-positive (\%FITC+), as outlined in Figure 6.3. 


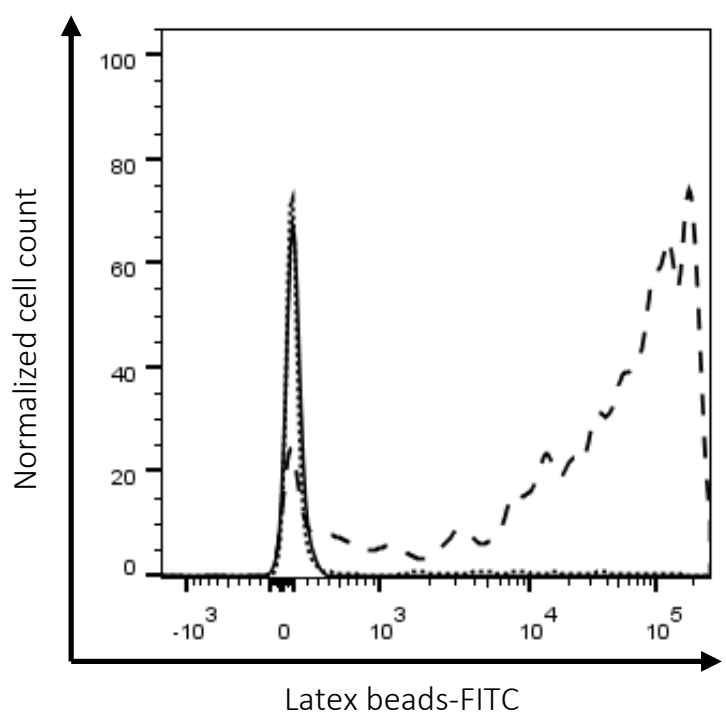

Figure 6.3 Representative plot of neutrophil phagocytosis.

Phagocytosis was measured by the \%FITC-positive neutrophils. Solid line, without latex beads; dotted line, unstimulated; dashed line, Pam3CSK4 stimulated $(100 \mathrm{ng} / \mathrm{mL})$. 


\subsubsection{Analysis of cell culture supernatant.}

Elastase secretion (Abcam) and IL-8 secretion (Thermo Fisher Scientific) in cell culture supernatant was measured by ELISA, as per manufacturer's instructions.

\subsubsection{Statistical analysis.}

In the previous study, we were able to detect a significant effect of platelets on markers of neutrophil function (CD66b expression, elastase secretion) with a sample size of 10, and the variances seen with these markers were narrower than the variances of other markers examined in this previous study. Therefore, a sample size of 10 was considered appropriate for this study. To examine changes in baseline (- platelets) neutrophil activation markers in response to TLR stimulation, differences in raw measurements were examined using one-way ANOVA with posthoc Sidak's multiple comparisons tests. To examine the effect of platelets on the neutrophil response to TLR stimulation, each baseline (- platelets) neutrophil only measurement was normalized to 1. Each platelet co-culture (+ platelets) measurement was then reported as a relative change, compared to the paired neutrophil only measurement (+ platelets measurement/platelets measurement). When examining the relative change data for unstimulated cultures (platelets vs. + platelets) for some of the activation markers (for example, elastase secretion and phagocytosis), we saw a shift away from a relative change of 1 in the presence of platelets. This indicated that platelets were having an effect on these markers in unstimulated cultures, and any relative change with platelets following TLR stimulation may be due to this baseline shift rather than an effect of stimulation. To account for this, relative change differences between neutrophil only (- platelets) and platelet co-culture (+ platelets) were examined using a repeated measures two-way ANOVA with post-hoc Sidak's multiple comparisons tests for all neutrophil markers. All statistical analyses were performed using GraphPad Prism 7 (GraphPad Software Inc.). 


\subsection{Results.}

\subsubsection{Platelets reduce CD66b expression in response to Pam3CSK4 and FSL-1.}

Analysis of baseline (- platelets) results for TLR stimulation showed an increase in CD66b expression in response to all three TLR agonists used in this study (Table 6.2). In response to lowdose $(1 \mathrm{ng} / \mathrm{mL})$ and high-dose $(100 \mathrm{ng} / \mathrm{mL})$ LPS, CD66b expression was $161 \%$ and $163 \%$ of the MFI recorded in unstimulated (US) cultures, respectively (both $p<0.05$ ). Similarly, CD66b expression was dose-dependently increased in response to Pam3CSK4 (low-dose, 162\% of US MFI, p=0.07; high-dose, 312\%, p<0.05) and FSL-1 (low-dose, 169\%; high-dose, 232\%; both $p<0.05$ ).

The raw CD66b expression levels (as measured by MFI) are shown in Figure 6.4A, and relative changes in CD66b expression in neutrophil-platelet co-cultures (+ platelets) are shown in Table 6.3 and Figure 6.4B. In examining these relative changes, we show that the increase in CD66b expression seen in response to high-dose Pam3CSK4 and FSL-1, but not LPS, was attenuated in the presence of platelets. In response to high-dose Pam3CSK4, the presence of platelets attenuated CD66b expression by $16 \%$ ( $p<0.0001)$. CD66b expression was also attenuated by $14 \%$ and $10 \%$ in the presence of platelets following stimulation with low-dose and high-dose FSL-1, respectively (both $\mathrm{p}<0.05)$. 
Table 6.2 Baseline (- platelets) geometric MFI of neutrophil activation markers in response to TLR agonism, compared to unstimulated cultures.

\begin{tabular}{|c|c|c|c|c|c|c|c|}
\hline \multirow[b]{2}{*}{ Agonist } & \multirow[b]{2}{*}{$\mathrm{ng} / \mathrm{mL}$} & \multicolumn{2}{|c|}{ CD66b expression } & \multicolumn{2}{|l|}{ CD11b expression } & \multicolumn{2}{|c|}{ CD62L expression } \\
\hline & & Absolute MFI & $\%$ of US & Absolute MFI & $\%$ of US & Absolute MFI & $\%$ of US \\
\hline US & -- & $2195(831)$ & -- & $3843(1085)$ & -- & $509(205)$ & -- \\
\hline \multirow[t]{2}{*}{ LPS } & 1 & $3539(1740)^{* *}$ & $161 \%$ & $6353(1465)^{* * *}$ & $165 \%$ & $318(99)^{* *}$ & $62 \%$ \\
\hline & 100 & $3574(2201)^{*}$ & $163 \%$ & $6248(1365)^{* * * *}$ & $163 \%$ & $279(65)^{* *}$ & $55 \%$ \\
\hline \multirow[t]{2}{*}{ Pam3CSK4 } & 1 & 3559 (1901) & $162 \%$ & $7428(3790)^{*}$ & $193 \%$ & $370(155)^{*}$ & $73 \%$ \\
\hline & 100 & $6856(4512)^{*}$ & $312 \%$ & $6873(2031)^{* *}$ & $179 \%$ & $284(113)^{* *}$ & $56 \%$ \\
\hline \multirow[t]{2}{*}{ FSL-1 } & 1 & $3720(1915)^{*}$ & $169 \%$ & $7079(3470)^{*}$ & $184 \%$ & $332(103)^{* *}$ & $65 \%$ \\
\hline & 100 & $5087(2554)^{* *}$ & $232 \%$ & $7158(1915)^{* * * *}$ & $186 \%$ & $255(45)^{* *}$ & $50 \%$ \\
\hline
\end{tabular}

Differences between US and each of the TLR stimulation conditions were examined by one-way ANOVA with post-hoc Sidak's multiple comparisons tests. ${ }^{*} p<0.05,{ }^{* *} p<0.01, * * * p<0.001, * * * * p<0.0001$ 
Table 6.3 Relative change in neutrophil activation markers in neutrophil-platelet co-culture (+ platelets) and following TLR stimulation.

\begin{tabular}{|l|l|l|l|l|}
\cline { 2 - 5 } \multicolumn{2}{|c|}{} & \multicolumn{3}{c|}{ +platelets $^{2}$} \\
Agonist & $\mathrm{ng} / \mathrm{mL}$ & CD66b expression & CD11b expression & CD62L expression \\
\hline US & -- & $0.97(0.03)$ & $0.95(0.14)$ & $1.07(0.10)^{*}$ \\
PPS & 1 & $0.92(0.14)$ & $0.99(0.21)$ & $0.97(0.15)$ \\
& 100 & $0.97(0.12)$ & $0.95(0.12)$ & $0.98(0.10)$ \\
FSL-1 & 1 & $0.95(0.13)$ & $0.88(0.11)^{* *}$ & $0.97(0.09)$ \\
& 1 & $0.84(0.13)^{* * * *}$ & $0.90(0.15)^{*}$ & $1.06(0.14)^{*}$ \\
& 1 & $0.86(0.10)^{* * *}$ & $0.87(0.16)^{* *}$ & $1.09(0.10)^{* * *}$ \\
& 100 & $0.90(0.07)^{*}$ & $0.88(0.13)^{* *}$ & $1.02(0.12)$ \\
\hline
\end{tabular}

\#all neutrophil only measurements were normalized to 1, and all co-culture (+ platelets) measurements were compared to this normalized response. Differences between these measurements were examined by twoway ANOVA and post-hoc Sidak's multiple comparisons tests. ${ }^{*} p<0.05,{ }^{*} p<0.01,{ }^{* * *} p<0.001$, $* * * * p<0.0001$. 
A

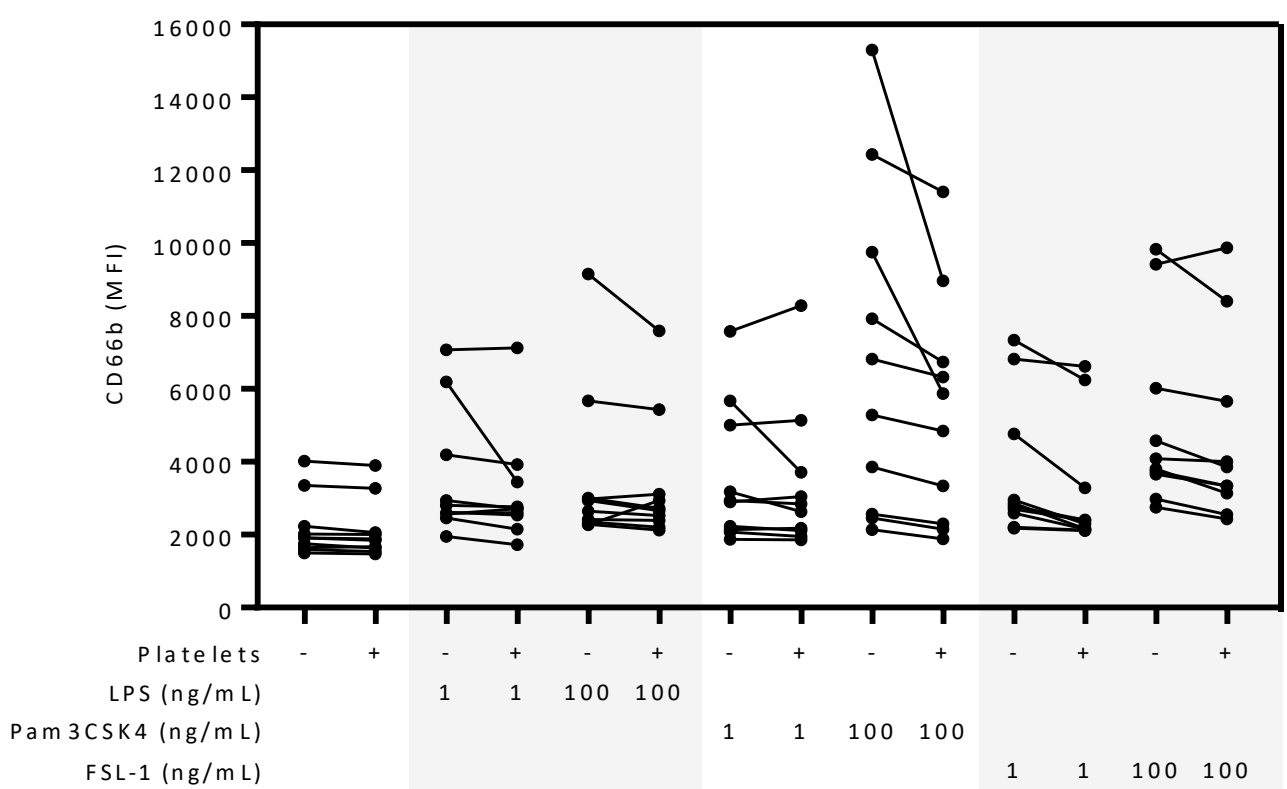

B

CD66b expression

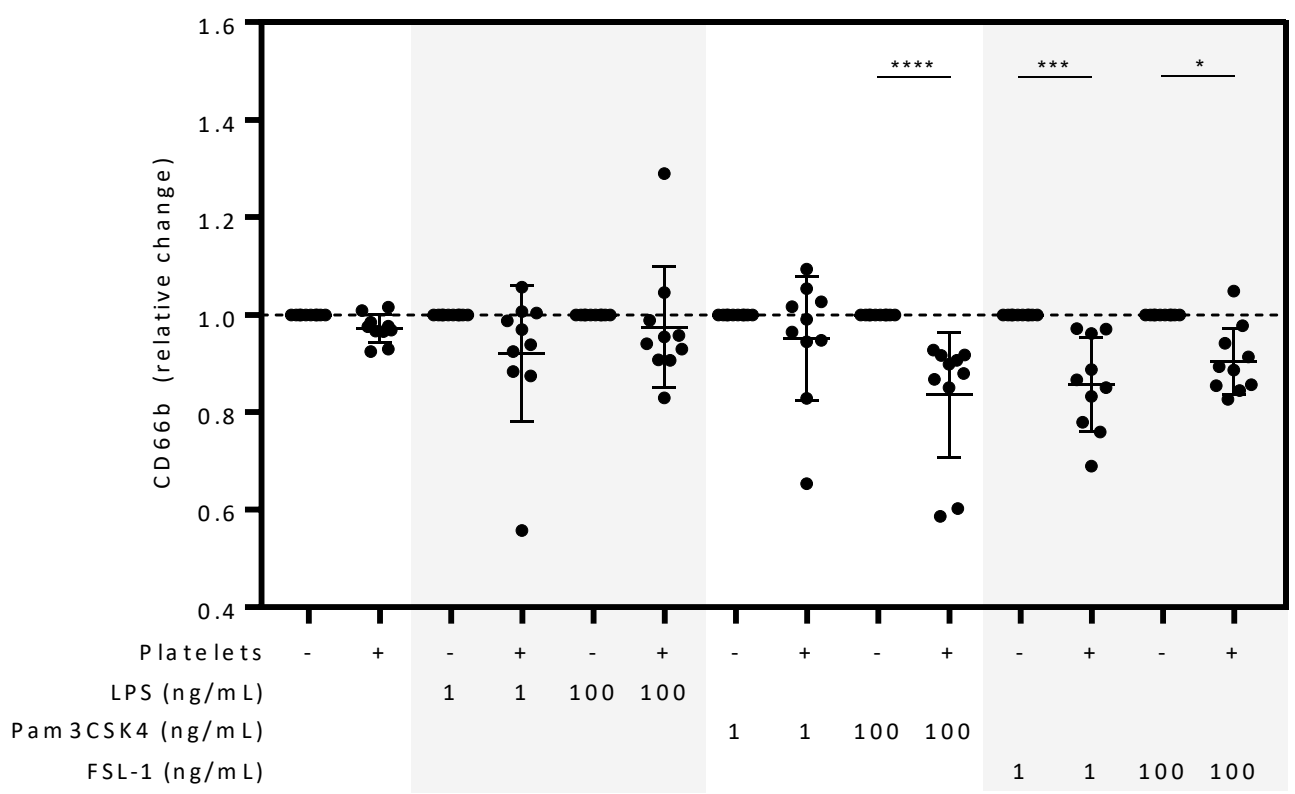

Figure 6.4 Platelets modulate expression of neutrophil CD66b expression in response to Pam3CSK4 and FSL-1.

Neutrophil CD66b expression was measured by MFI without platelets (- platelets) and in co-culture (+ platelets) with no stimulation and in response to 1 and 100 ng/mL LPS, Pam3CSK4 and FSL-1 (A). For each co-culture measurement, CD66b expression was calculated as a relative change (B). Differences between these relative change measurements were examined were examined by two-way ANOVA and post-hoc Sidak's multiple comparisons tests. ${ }^{*} p<0.05$, $* * * p<0.001, * * * * p<0.0001$. 


\subsubsection{Platelets modulate neutrophil CD11b expression following stimulation with Pam3CSK4 and FSL-1.}

At baseline (- platelets), we show a significant and relatively consistent increase in CD11b expression in response to TLR stimulation (Table 6.2). With low-dose and high-dose LPS stimulation, CD11b expression was $165 \%$ and $163 \%$ of the expression levels seen in unstimulated cultures, respectively (both $\mathrm{p}<0.001$ ). Similarly, neutrophil CD11b expression increased in response to lowdose (193\% of US MFI) and high-dose (179\%) Pam3CSK4 and in response to low-dose (184\%) and high-dose (186\%) FSL-1 (all both $p<0.05)$.

CD11b expression, as measured by MFI (Figure 6.5A) and expressed as a relative change (Figure 6.5B, Table 6.3), is shown below. When analysing the relative change in neutrophil-platelet cocultures, we show that the increase in CD11b expression following stimulation with Pam3CSK4 and FSL-1 is attenuated in the presence of platelets. With platelets, CD11b expression was attenuated by $12 \%$ and $10 \%$ in response to low-dose and high-dose Pam3CSK4, respectively (both $p<0.05$ ). Similarly, in platelet co-cultures, CD11b expression was attenuated following FSL-1 stimulation (low-dose, 13\% reduction; high-dose, $12 \%$ reduction; both $\mathrm{p}<0.01$ ). Platelets did not alter CD11b expression in response to either dose of LPS. 


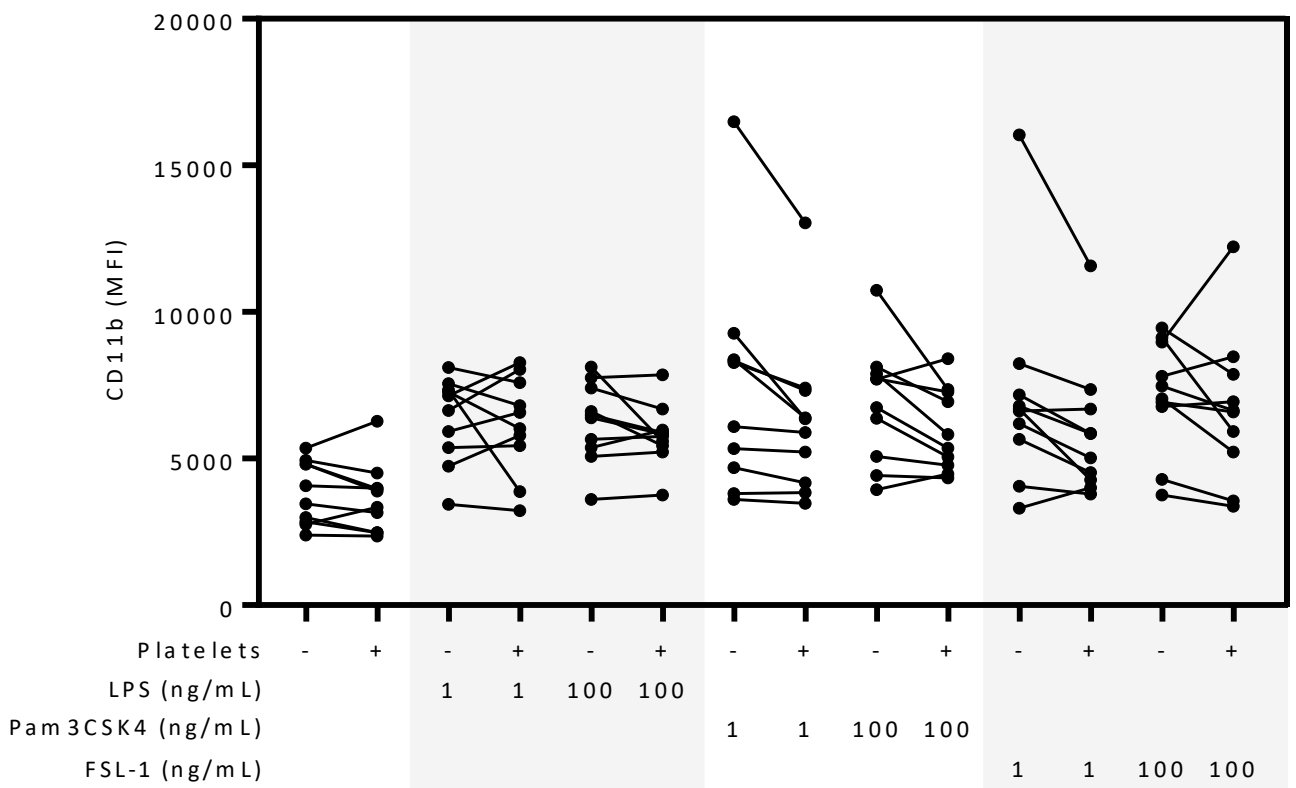

B

CD 11 b expression

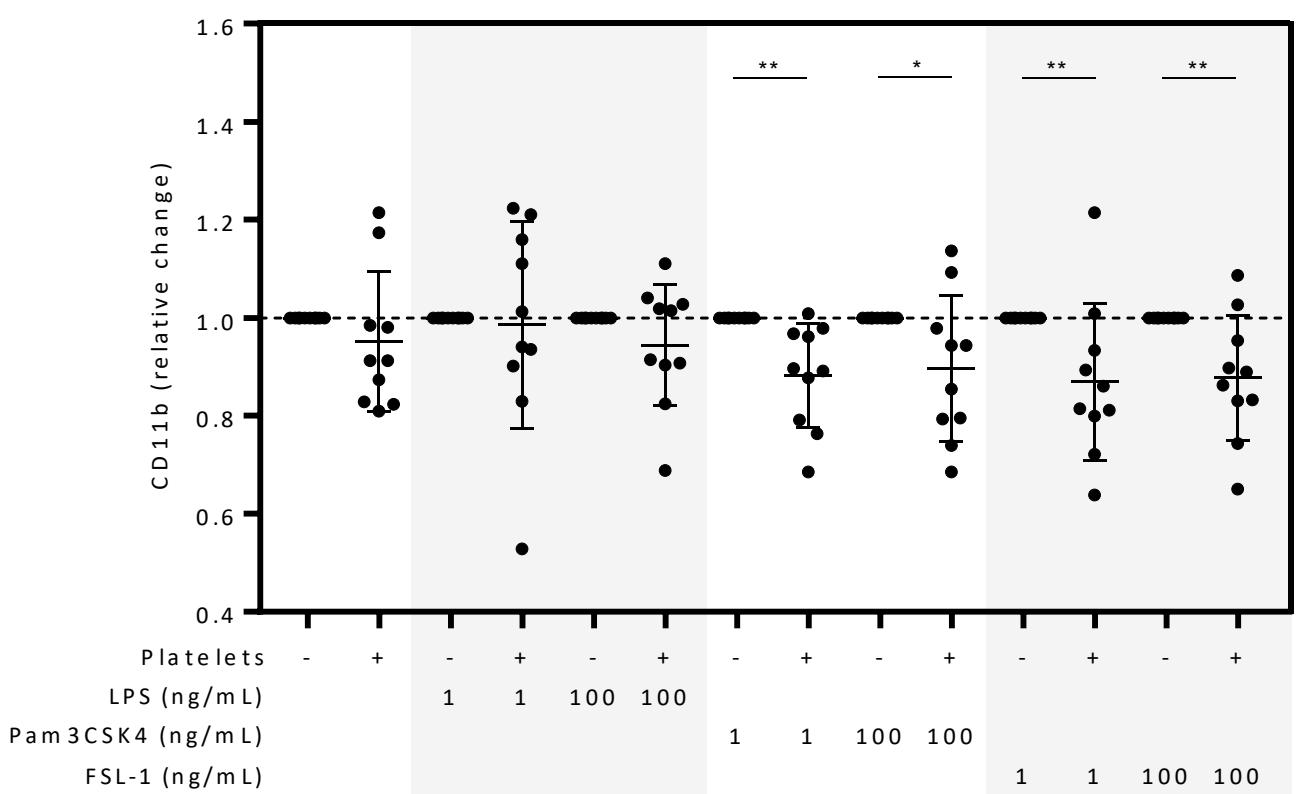

Figure 6.5 CD11b expression is altered by platelets in response to Pam3CSK4 and FSL-1.

Neutrophil CD11b expression was measured by MFI with and without platelets in unstimulated cultures and in response to LPS, Pam3CSK4 and FSL-1 (A). CD11b expression for each co-culture measurement was calculated as a relative change (B). Differences were examined were examined by two-way ANOVA and post-hoc Sidak's multiple comparisons tests. ${ }^{*} p<0.05,{ }^{* *} p<0.01$. 


\subsubsection{Platelets modulate neutrophil CD62L expression in unstimulated cultures and in response to some TLR stimulation conditions.}

CD62L shedding is a well-characterized neutrophil activation marker, and attenuated expression indicates increased neutrophil activation. Considering baseline (- platelets) CD62L expression, we see a significant dose-dependent reduction in CD62L expression following stimulation with LPS (low-dose, 62\% of US MFI; high-dose, 55\%), Pam3CSK4 (low-dose, 73\%; high-dose, 56\%) and FSL1 (low-dose, 65\%; high-dose, 50\%) (all p<0.05; Table 6.2).

When analysing the effects of platelet co-culture, which are shown in Figure 6.6, we see that CD62L expression is significantly increased by $7 \%$ in unstimulated cultures $(p<0.05)$. Similarly, CD62L levels increased by 6\% in response to high-dose Pam3CSK4 and increased by $9 \%$ in response to low-dose FSL-1 (both $\mathrm{p}<0.05$ ). The presence of platelets did not affect CD62L expression under any other stimulation condition. 
A

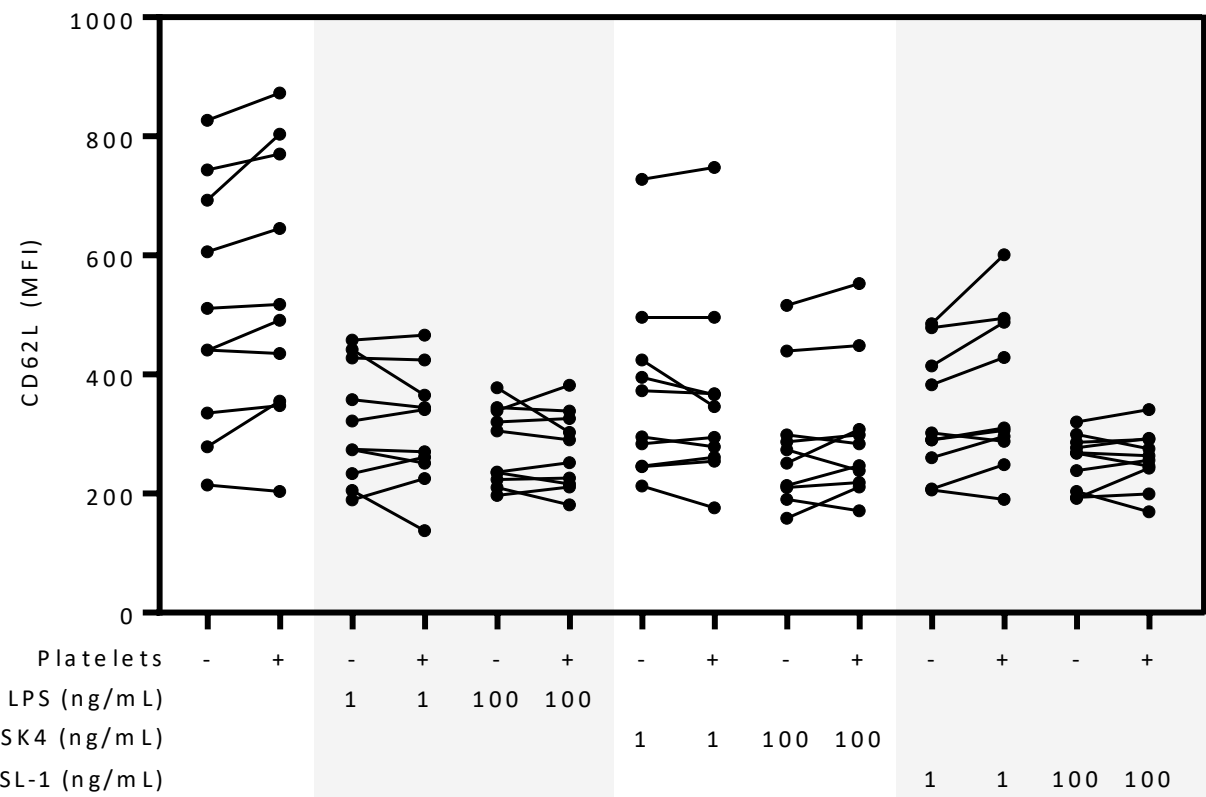

B

CD 62 L expression

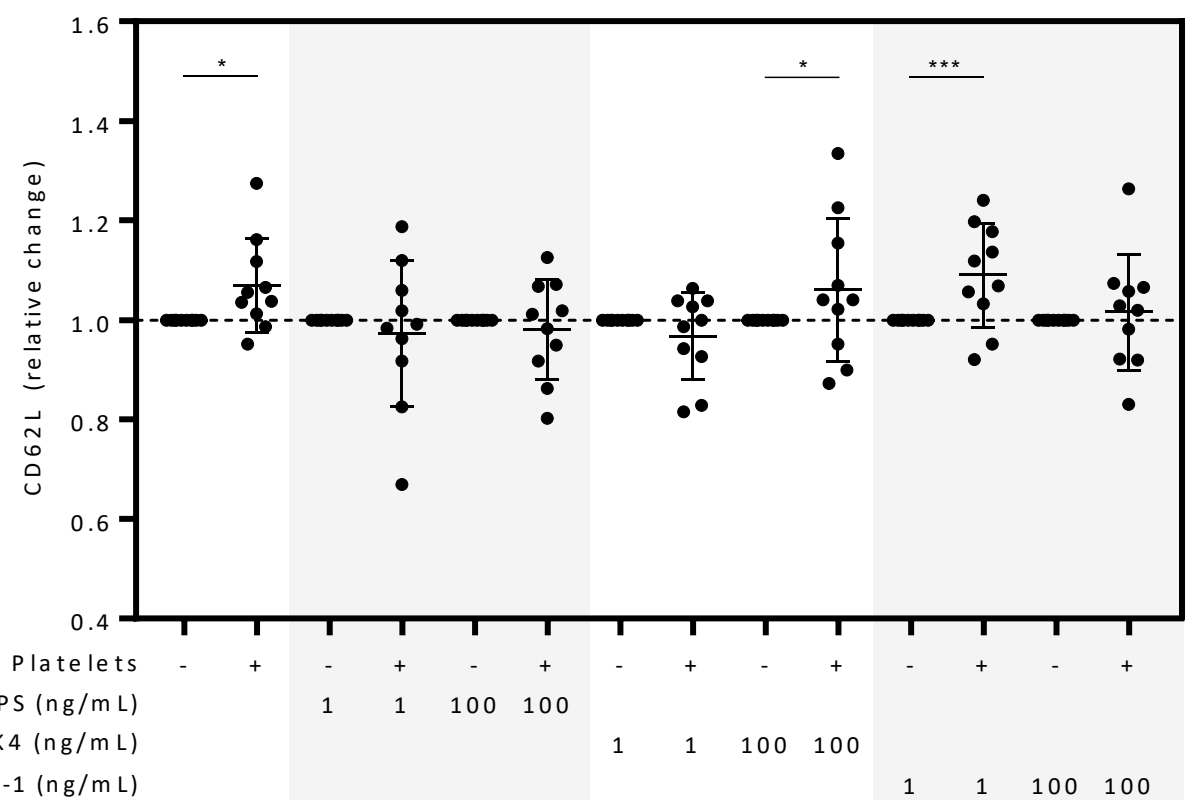

Figure 6.6 CD62L expression is altered by platelets in unstimulated cultures and in response to some TLR stimuli.

Neutrophil CD62L expression was measured by MFI with and without platelets in unstimulated cultures and in response to LPS, Pam3CSK4 and FSL-1 (A). CD62L expression for each co-culture measurement was calculated as a relative change (B). Differences were examined were examined by two-way ANOVA and post-hoc Sidak's multiple comparisons tests. ${ }^{*} p<0.05,{ }^{* * *} p<0.001$. 


\subsubsection{With platelets, neutrophil elastase production was attenuated in unstimulated cultures and in response to low-dose TLR stimulation.}

Elastase secretion was assessed from the supernatant of neutrophil-only and neutrophil-platelet co-cultures. At baseline (- platelets), elastase secretion significantly increased in response to low doses of LPS (146\% of US level), Pam3CSK4 (134\%) and FSL-1 (162\%; all p<0.05; Table 6.4). Elastase secretion in response to high-dose TLR stimulation was numerically higher, but not statistically significantly higher, than in unstimulated cultures.

The raw elastase secretion levels are shown in Figure 6.7A, and relative changes with platelet coculture are shown in Table 6.5 and Figure 6.7B. With the addition of platelets, elastase secretion was significantly lowered by $18 \%$ in unstimulated cultures $(p<0.01)$, and the increase in elastase secretion following low-dose TLR stimulation was also attenuated by platelets (LPS, 17\% reduction; Pam3CSK4, 20\%; FSL-1, 19\%; all p<0.05). 
Table 6.4 Baseline (- platelets) levels of neutrophil secretory products in response to TLR agonism, compared to unstimulated cultures.

\begin{tabular}{|l|l|ll|ll|}
\cline { 3 - 6 } \multicolumn{2}{|c|}{} & \multicolumn{3}{l|}{ Elastase secretion } & IL-8 secretion \\
\hline Agonist & $\mathrm{ng} / \mathrm{mL}$ & Absolute $\mathrm{ng} / \mathrm{mL}$ & $\%$ of US & Absolute $\mathrm{pg} / \mathrm{mL}$ & $\%$ of US \\
\hline US & -- & $32.5(13.6)$ & -- & $41.6(38.9)$ & -- \\
LPS & 1 & $47.3(23.4)^{*}$ & $146 \%$ & $114.4(74.3)^{* *}$ & $275 \%$ \\
& 100 & $37.5(15.3)$ & $115 \%$ & $176.6(85.1)^{* * *}$ & $425 \%$ \\
Pam3CSK4 & 1 & $43.6(19.3)^{*}$ & $134 \%$ & $103.3(91.9)^{*}$ & $248 \%$ \\
& 100 & $36.9(15.6)$ & $114 \%$ & $175.0(152.8)^{*}$ & $421 \%$ \\
FSL-1 & 1 & $52.7(19.9)^{* * *}$ & $162 \%$ & $87.2(69.4)^{*}$ & $210 \%$ \\
& 100 & $48.0(23.8)$ & $148 \%$ & $130.0(87.7)^{* *}$ & $313 \%$ \\
\hline
\end{tabular}

Differences between US and each of the TLR stimulation conditions were examined by one-way ANOVA with posthoc Sidak's multiple comparisons tests. ${ }^{*} p<0.05,{ }^{* *} p<0.01,{ }^{* * *} p<0.001$.

Table 6.5 Relative change in neutrophil secretory products in neutrophil-platelet co-culture (+ platelets) and following TLR stimulation.

\begin{tabular}{|l|l|l|l|}
\cline { 3 - 4 } \multicolumn{2}{|c|}{} & \multicolumn{2}{c|}{ platelets $^{\#}$} \\
\hline Agonist & $\mathrm{ng} / \mathrm{mL}$ & Elastase secretion & IL-8 secretion \\
\hline US & -- & $0.82(0.13)^{* *}$ & $0.94(0.14)$ \\
LPS & 1 & $0.83(0.14)^{*}$ & $1.28(0.28)^{* * *}$ \\
& 100 & $0.93(0.31)$ & $1.00(0.18)$ \\
& 1 & $0.80(0.25)^{* * *}$ & $1.17(0.43)$ \\
FSL-1 & 100 & $0.89(0.15)$ & $0.94(0.30)$ \\
& 1 & $0.81(0.13)^{* *}$ & $1.05(0.51)$ \\
& 100 & $0.89(0.13)$ & $0.99(0.14)$ \\
\hline
\end{tabular}

\#all neutrophil only measurements were normalized to 1 , and all co-culture (+ platelets) measurements were compared to this normalized response. Differences between these measurements were examined by two-way ANOVA and post-hoc Sidak's multiple comparisons tests. ${ }^{*} p<0.05,{ }^{* *} p<0.01^{* * *} p<0.001$. 
A

Elastase secretion

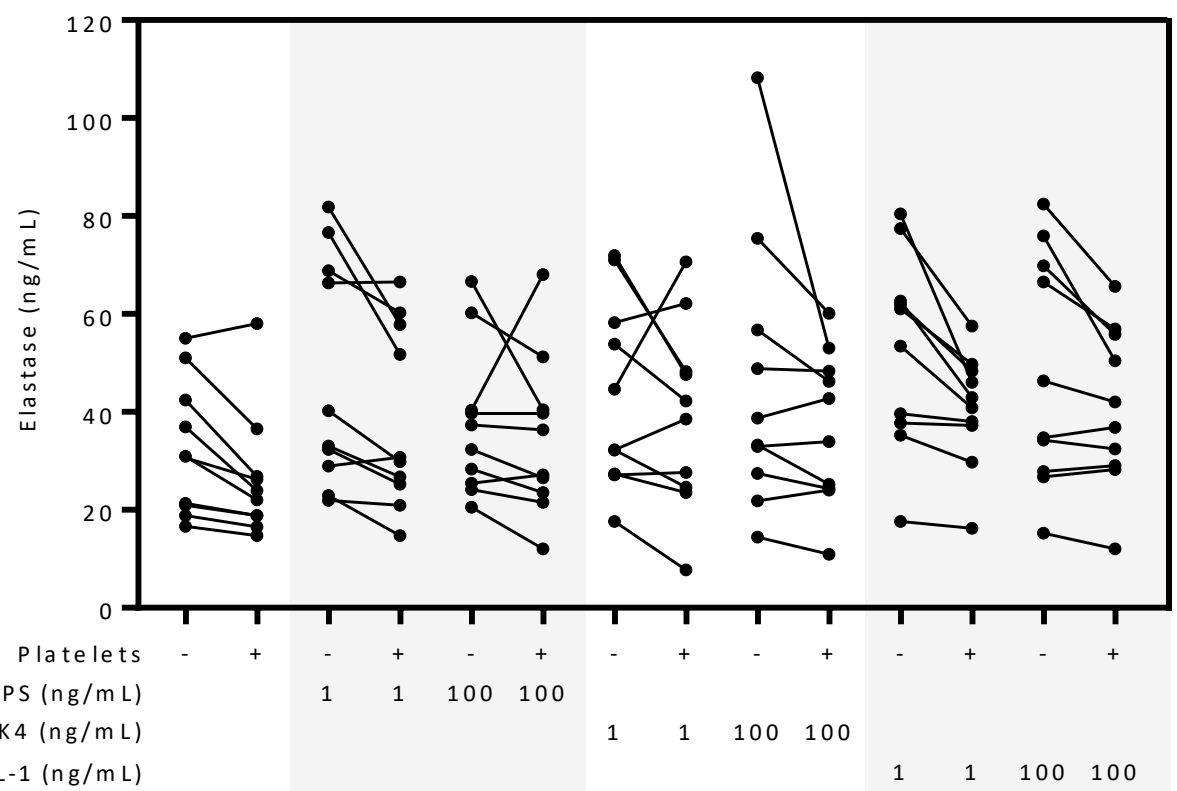

B

Elastase secretion

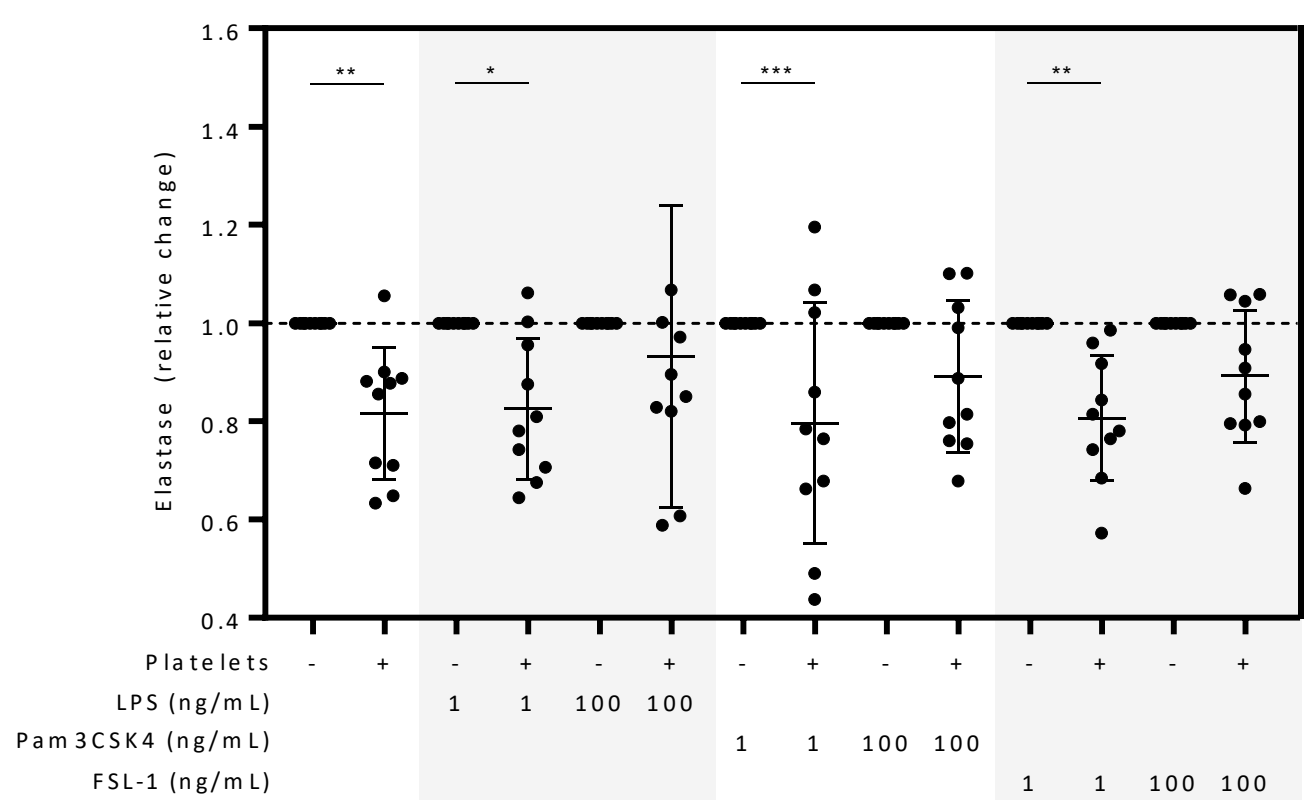

Figure 6.7 Platelets attenuate elastase secretion in unstimulated cultures and in response to low-dose TLR stimulation.

Elastase secretion $(\mathrm{ng} / \mathrm{mL})$ was measured with and without platelets in unstimulated cultures and in response to LPS, Pam3CSK4 and FSL-1 (A). Elastase secretion for each co-culture measurement was calculated as a relative change (B). Differences were examined were examined by two-way ANOVA and post-hoc Sidak's multiple comparisons tests. ${ }^{*} p<0.05,{ }^{* *} p<0.01,{ }^{* * *} p<0.001$. 


\subsubsection{Neutrophil IL-8 production is decreased in the presence of platelets following low-dose LPS stimulation.}

There was a significant increase in baseline IL-8 secretion in response to TLR agonism, compared to unstimulated cultures (Table 6.4). In response to LPS, IL-8 secretion was $275 \%$ (low-dose) and $425 \%$ (high-dose) of the level of secretion seen in unstimulated cultures (both $p<0.01$ ), and this pattern of secretion was similar for Pam3CSK4 (low-dose, 248\% of US levels; high-dose, 421\%; both $p<0.05$ ) and FSL-1 (low-dose, 210\%; high-dose, 313\%; both $p<0.05$ ).

The presence of platelets only significantly altered IL-8 secretion in response to low-dose LPS: the increase in IL-8 secretion with this dose was further increased by $28 \%(p<0.001$; Table 6.5 and Figure 6.8). Platelets did not affect IL-8 secretion in unstimulated cultures or in response to either Pam3CSK4 or FSL-1. 
A

IL-8 secretion

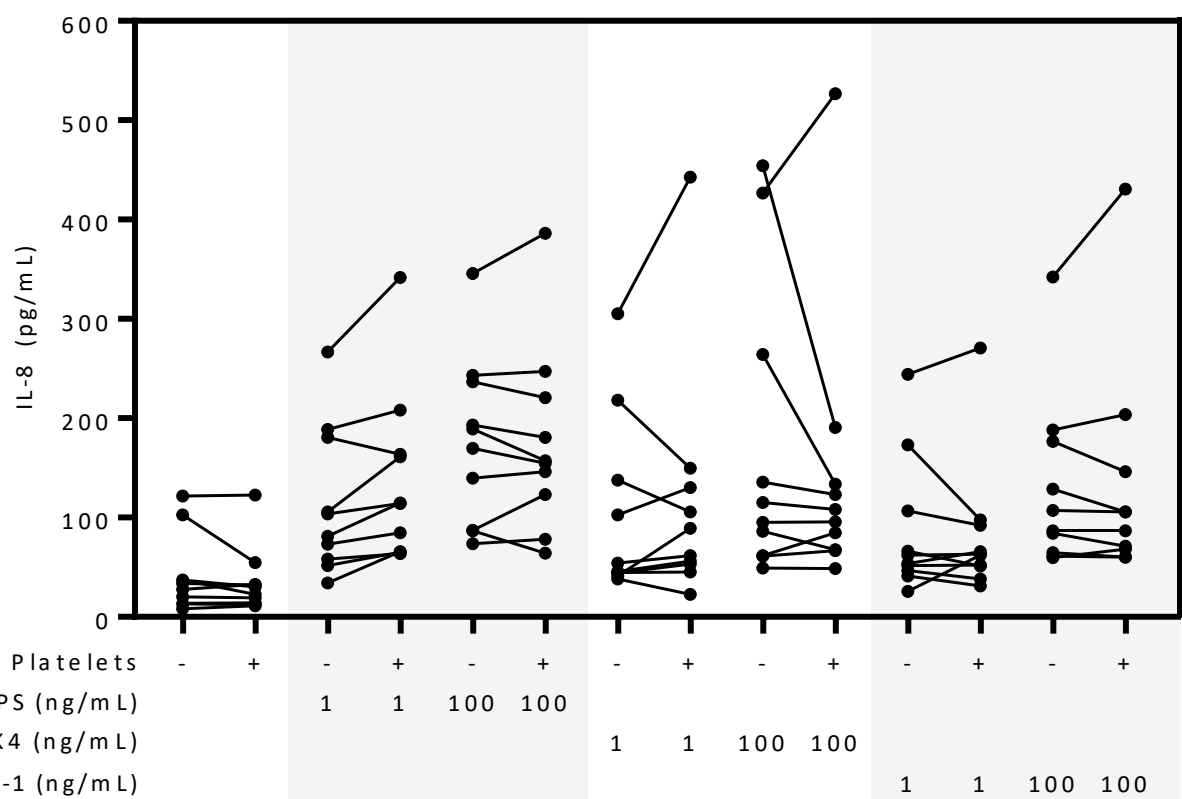

B

IL-8 secretion

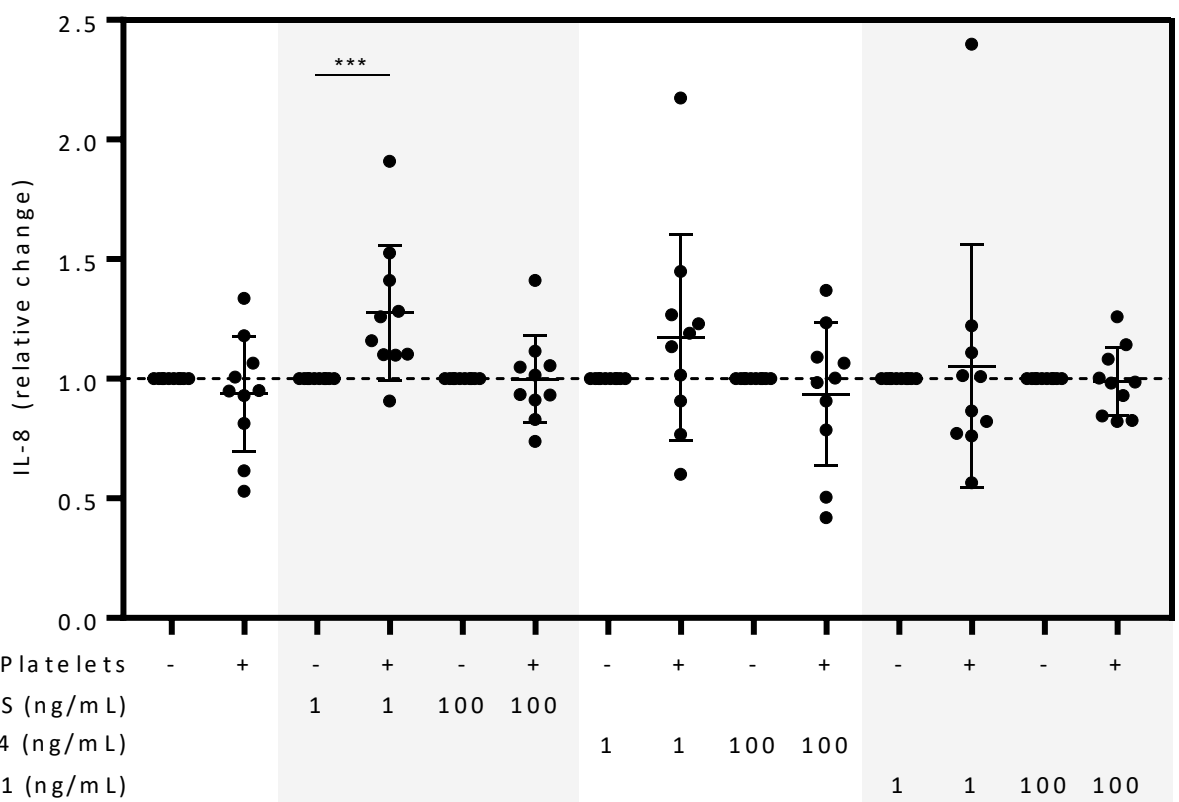

Figure 6.8 Platelets modulate IL-8 secretion only in response to low-dose LPS.

IL-8 secretion $(\mathrm{pg} / \mathrm{mL})$ was measured with and without platelets in unstimulated cultures and in response to LPS, Pam3CSK4 and FSL-1 (A). IL-8 secretion for each co-culture measurement was calculated as a relative change (B). Differences were examined were examined by two-way ANOVA and post-hoc Sidak's multiple comparisons tests. $* * * p<0.001$. 


\subsubsection{The addition of platelets modulates neutrophil phagocytosis in unstimulated cultures and in response to some TLR stimulation conditions.}

At baseline (- platelets), phagocytosis significantly and uniformly increased in response to highdose TLR stimulation (LPS, 69.9\% of the neutrophil population was FITC+; Pam3CSK4, 69.4\%; FSL1, 69.7\%; all $p<0.0001$ ) compared to unstimulated cultures (22.9\%; Table 6.6). Phagocytosis was similarly increased following low-dose LPS stimulation (70.6\%, p<0.0001). Stimulation with a low dose of either Pam3CSK4 or FSL-1 also produced a significant increase in phagocytic activity that was consistent across both doses (Pam3CSK4, 40.3\%; FSL-1, 41.8\%; both $p<0.01$ ).

With the addition of platelets, the phagocytic activity of neutrophils was increased by $121 \%$ in unstimulated cultures $(p<0.0001$, Table 6.7, Figure 6.9). Also, the increase in phagocytosis seen in response to low-dose Pam3CSK4 was further increased by $35 \%(p<0.05)$ and in response to lowdose FSL-1 was further increased by $31 \%(p=0.08)$. Platelets did not further increase phagocytic activity in response to any dose of LPS, nor in response to high doses of Pam3CSK4 and FSL-1. 
Table 6.6 Baseline (- platelets) neutrophil phagocytosis, as measured by the percentage of FITC-positive neutrophils (\%FITC+), in response to TLR agonism compared to unstimulated cultures.

\begin{tabular}{|c|c|c|}
\hline Agonist & $\mathrm{ng} / \mathrm{mL}$ & \%FITC+ neutrophils \\
\hline US & -- & $22.9(10.0)$ \\
\hline \multirow[t]{2}{*}{ LPS } & 1 & $70.6(14.4)^{* * * *}$ \\
\hline & 100 & $69.9(14.1)^{* * * *}$ \\
\hline \multirow[t]{2}{*}{ Pam3CSK4 } & 1 & $40.3(15.3)^{* *}$ \\
\hline & 100 & $69.4(24.9)^{* * *}$ \\
\hline \multirow[t]{2}{*}{ FSL-1 } & 1 & $41.8(10.7)^{* * * *}$ \\
\hline & 100 & $69.7(14.8) * * * *$ \\
\hline \multicolumn{3}{|c|}{$\begin{array}{l}\text { Differences between US and each of the TLR } \\
\text { stimulation conditions were examined by one-way } \\
\text { ANOVA with post-hoc Sidak's multiple comparisons } \\
\text { tests. }{ }^{* *} p<0.01,{ }^{* *} p<0.001,{ }^{* * *} p<0.0001 \text {. }\end{array}$} \\
\hline
\end{tabular}

Table 6.7 Relative change in phagocytosis (\%FITC+ neutrophils) in neutrophil-platelet co-culture (+ platelets) and following TLR stimulation.

\begin{tabular}{|l|l|l|}
\hline Agonist & $\mathrm{ng} / \mathrm{mL}$ & + platelets $^{\#}$ \\
\hline US & -- & $2.21(0.95)^{* * * *}$ \\
& 1 & $0.95(0.14)$ \\
PPS & 100 & $0.98(0.18)$ \\
& 1 & $1.35(0.32)^{*}$ \\
FSL-1 & 100 & $0.97(0.15)$ \\
& 1 & $1.31(0.43)$ \\
& 100 & $0.97(0.28)$ \\
\hline
\end{tabular}

\#all neutrophil only measurements were normalized to 1 , and all co-culture (+ platelets) measurements were compared to this normalized response. Differences between these measurements were examined by twoway ANOVA and post-hoc Sidak's multiple comparisons tests. ${ }^{*} p<0.05, * * * * p<0.0001$. 
A

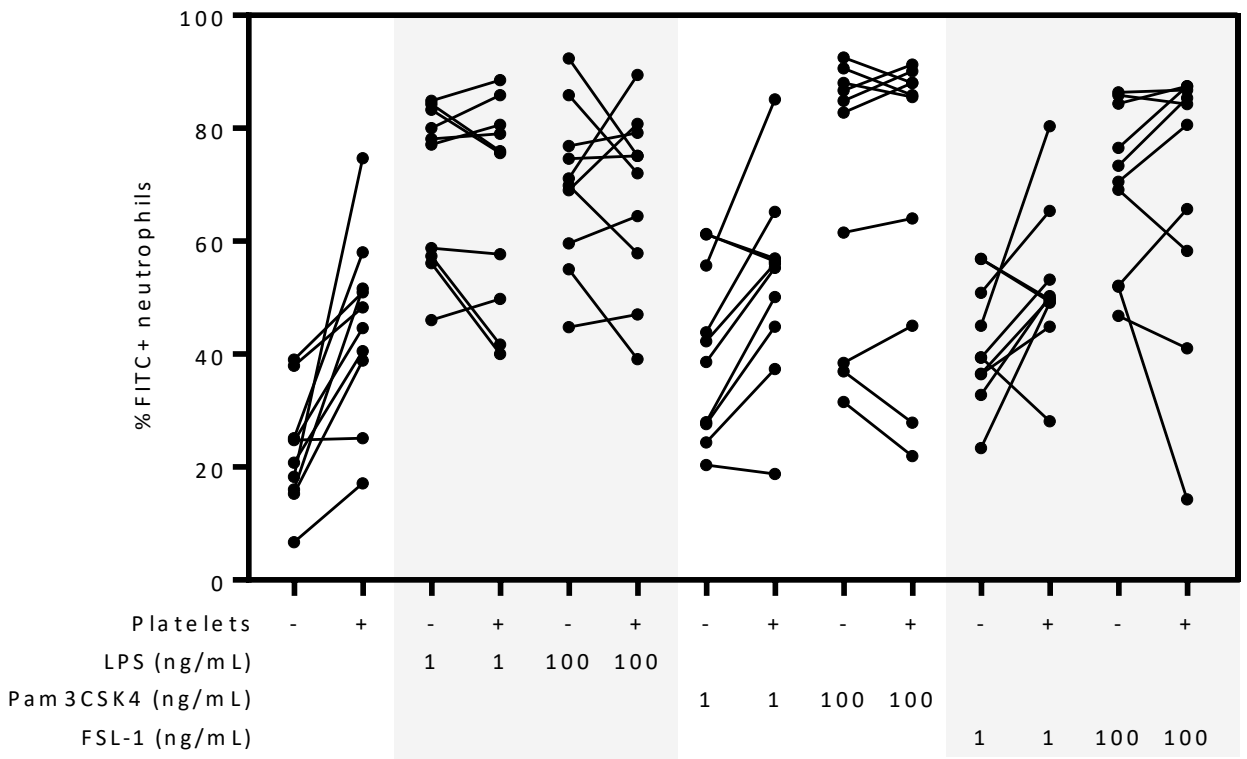

B Phagocytosis

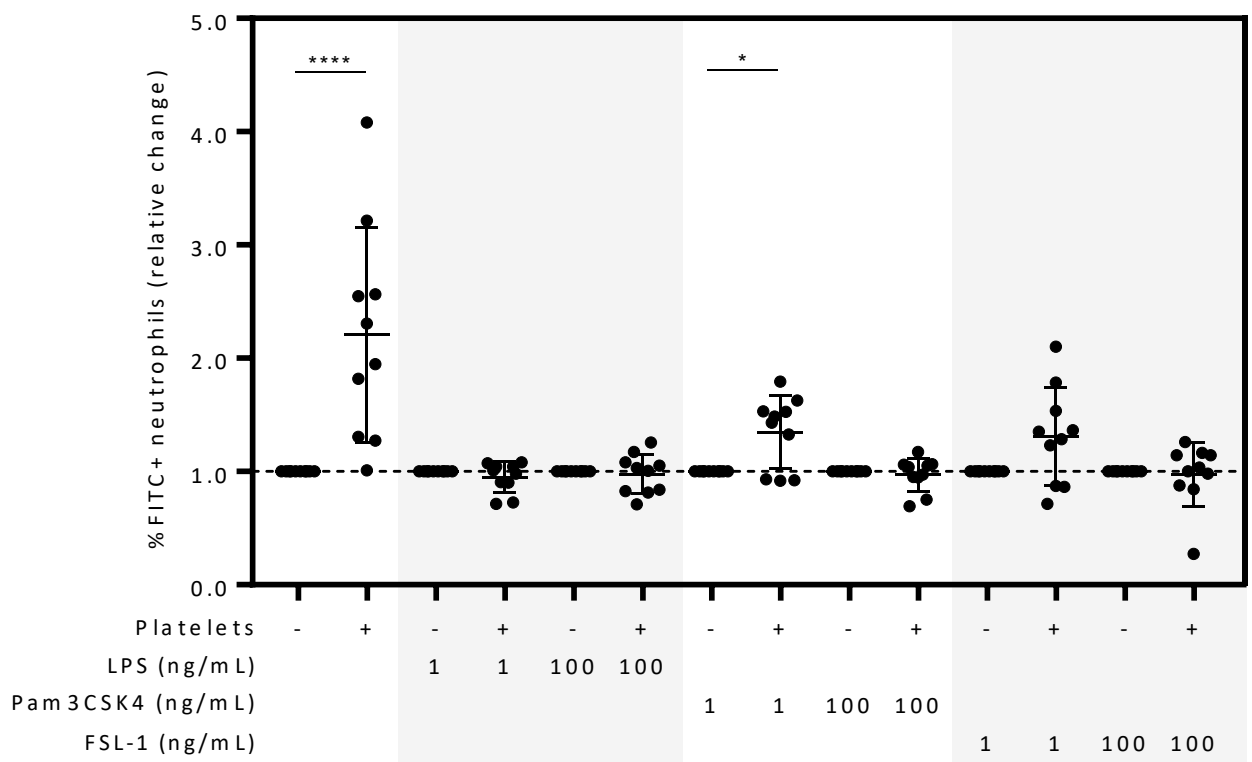

Figure 6.9 Neutrophil phagocytosis is altered by platelets in unstimulated cultures and following low-dose Pam3CSK4.

The phagocytic activity of neutrophils, as measured by the \%FITC+ neutrophils, was assessed in culture with and without platelets with no stimulation and in response to LPS, Pam3CSK4 and FSL-1 (A). Phagocytosis for each coculture measurement was calculated as a relative change (B). Differences were examined were examined by two-way ANOVA and post-hoc Sidak's multiple comparisons tests. ${ }^{*} p<0.05,{ }^{* * * *} p<0.0001$. 


\subsection{Discussion.}

We demonstrate, here, that platelets differentially modulate several neutrophil functions in a TLR agonist-specific and dose-specific manner. The influence of platelets on the expression of three well-characterized flow cytometric markers of neutrophil activation were assessed in this study. We showed that CD66b and CD11b expression increased, and CD62L expression decreased (CD62L is shed from the neutrophil surface) following TLR stimulation, indicating effective neutrophil activation. Firstly, we showed that the increase in neutrophil CD66b expression seen in response to high-dose (100 ng/mL) Pam3CSK4 and in response to both doses (1 and $100 \mathrm{ng} / \mathrm{mL}$ ) of FSL-1 was significantly attenuated in the presence of platelets. Secondly, CD11b expression was attenuated by platelets, regardless of dosage, in response to both Pam3CSK4 and FSL-1. Thirdly, in unstimulated cultures and following stimulation with high-dose Pam3CSK4 and low-dose FSL-1, CD62L expression was significantly increased in the presence of platelets. Platelets did not alter the expression of any activation marker in response to LPS. We also assessed the effect of platelets on two neutrophil secretory products: elastase secretion increased only in response to low-dose TLR agonism, and IL-8 secretion increased dose-dependently in response to TLR agonism. Firstly, we showed that elastase secretion was attenuated in the presence of platelets in unstimulated cultures as well as in response to low-dose stimulation with all three TLR agonists. Secondly, we showed that the increase in IL-8 secretion in response to low-dose LPS was further increased in the presence of platelets. Lastly, we showed that neutrophil phagocytosis significantly increased in response to TLR stimulation. In the presence of platelets, the phagocytic activity of neutrophils was increased in unstimulated cultures and in response to low-dose Pam3CSK4 and FSL-1. The results of this study are summarized in Table 6.8 below. In conclusion, platelets modify a number of neutrophil functions in both the absence of stimulation and in response to TLR stimulation. Furthermore, platelets can attenuate some, and simultaneously augment other, neutrophil functions. 
Table 6.8 Summary of the effect of platelets on the markers of neutrophil function assessed in this study.

\begin{tabular}{|c|c|c|c|c|c|c|c|}
\hline \multirow[b]{2}{*}{ Agonist } & \multirow[b]{2}{*}{$\mathrm{ng} / \mathrm{mL}$} & \multicolumn{6}{|c|}{ +platelets } \\
\hline & & CD66b & $\mathrm{CD} 11 \mathrm{~b}$ & CD62L & Elastase & IL-8 & Phagocytosis \\
\hline US & -- & & & $\uparrow$ & $\downarrow$ & & $\uparrow$ \\
\hline \multirow[t]{2}{*}{ LPS } & 1 & & & & $\downarrow$ & $\uparrow$ & \\
\hline & 100 & & & & & & \\
\hline \multirow[t]{2}{*}{ Pam3CSK4 } & 1 & & $\downarrow$ & & $\downarrow$ & & $\uparrow$ \\
\hline & 100 & $\downarrow$ & $\downarrow$ & 个 & & & \\
\hline \multirow[t]{2}{*}{ FSL-1 } & 1 & $\downarrow$ & $\downarrow$ & $\uparrow$ & $\downarrow$ & & $\uparrow \S$ \\
\hline & 100 & $\downarrow$ & $\downarrow$ & & & & \\
\hline
\end{tabular}

Orange shaded/ 个 Significant increase, blue shaded/ $\downarrow$ significant decrease in relative change. ${ }^{\S}$ Not statistically significant.

This study was conducted to further investigate some of the findings from Chapter 5: we have previously demonstrated that platelets reduce CD66b expression in response to Pam3CSK4 and FSL-1, but not LPS, and also reduce elastase secretion in response to a low dose of all three of these TLR agonists. We are the first to demonstrate that platelets can modulate neutrophil function in response to TLR agonism, and others have also demonstrated a dampening effect of platelets in neutrophil-platelet co-cultures stimulated with non-TLR agonists $[29,309]$ and in a model of infection [18]. In this study, we have expanded on our results from Chapter 5 by examining a larger number of markers of neutrophil function. Our aim was to determine whether platelets consistently dampen, or whether platelets differentially regulate, neutrophil responses to TLR stimulation. We conclude that platelets attenuate some markers of neutrophil function and augment other markers, and the platelet effect is dependent on the type and dosage of the TLR stimulus. Furthermore, platelets were able to modulate some markers in the absence of stimulation. It is interesting to note that, in a majority of marker of neutrophil function (CD66b, CD11b and CD62L expression, elastase secretion), platelets had a dampening effect. 
These results contribute to a growing body of evidence which suggests that platelets play a dual role in inflammation: platelets have traditionally been considered pro-inflammatory [21, 305], although we now understand these cells to be important immunoregulators that provide important anti-inflammatory cues to limit host damage (For a review, see [22]). Our results from this study and from Chapter 5, alongside a number of other studies [23, 24, 27, 306], indicate that platelets can limit inflammation by dampening leukocyte-mediated pro-inflammatory processes. Platelets are postulated to provide these cues to limit potential inflammatory host damage that can occur during excessive inflammation, such as during sepsis. Alongside attenuating some neutrophil responses, we also show that platelets can simultaneously augment other neutrophil responses. These results suggest that platelets are able to finely tune, rather than broadly suppress, neutrophil function. We also note that the platelet effect on these markers of neutrophil function was moderate, rather than completely inhibitory. Neutrophils are highly reactive and short-lived cells that are crucial to anti-microbial host defence, but have also been implicated in causing inflammatory host damage $[326,327]$. Therefore, the inflammatory response must be rapid and robust, but also must be tightly controlled to prevent rampant inflammation. We suggest that platelets are regulators of neutrophil function and, within this role, platelets can act as a brake to neutrophil-mediated inflammation. In this regard, the platelet effect can be considered a mechanism for inflammation control.

We are the first to demonstrate that platelets are able to modulate neutrophil responses to TLR stimulation, and previous studies have also demonstrated a similar platelet effect on neutrophil function in response to non-TLR agonists. For example, the production of ROS by neutrophils in response to fMLP has been shown to be attenuated by pre-treatment with resting platelets, and this effect was reversed by administration with aspirin or clopidogrel [309]. Others have observed a similar effect of platelets on ROS production with neutrophil-platelet co-culture [328, 329]. Furthermore, stimulated platelet supernatant, but not resting platelet supernatant, attenuated neutrophil chemotaxis and aggregation in response to concanavalin A and phorbol myristate acetate, and this effect was dependent on the amount of platelet supernatant added [29]. One study has also shown a dampening effect of platelets on neutrophil function in response to 
infectious stimuli: loss of a single platelet glycoprotein (GP Ib-IX) was sufficient to increase neutrophil CD11b expression in a mouse mode of sepsis, demonstrating a mechanism by which platelets can suppress neutrophil CD11b under highly pro-inflammatory conditions [18].

Neutrophil CD66b expression was one of the flow cytometric markers used in this study to assess the effect of platelets on neutrophil activation in response to TLR stimulation. Surface expression of CD66b is, more specifically, a marker of neutrophil degranulation [330, 331]. At baseline (in the absence of platelets), neutrophil CD66b expression substantially increased following stimulation with all three TLR agonists. The increase in CD66b expression in response to LPS was relatively similar across both doses ( $161 \%$ and $163 \%$ of unstimulated MFI), but a dose-dependent increase in CD66b expression was seen in response to both Pam3CSK4 and FSL-1. It may be that doses of 1 $\mathrm{ng} / \mathrm{mL}$ and $100 \mathrm{ng} / \mathrm{mL}$ of LPS saturate TLR4 signalling in neutrophils, such that CD66b expression is upregulated to a maximum level in response to these doses. Dose-dependent increases in CD66b expression may be seen at doses below $1 \mathrm{ng} / \mathrm{mL}$. In this study, the increase in CD66b expression in response to high-dose Pam3CSK4 and both doses of FSL-1 was significantly attenuated with the addition of platelets. These results were consistent with our findings in Chapter 5 . The platelet effect was relatively consistent across Pam3CSK4 (16\% reduction) and FSL-1 (14\%, 10\% reductions) and no effect was evident in unstimulated cultures. We are the first to report that platelets can consistently attenuate neutrophil CD66b expression, and this effect is dependent on the type and dosage of the TLR stimulus.

Alongside CD66b, expression of CD11b was also used as a marker of neutrophil activation in this study. Increased CD11b expression indicates an increased ability of neutrophils to transmigrate from the circulation into inflamed tissue, via binding to endothelial-bound ICAMs [332] and also indicates an increased ability to participate in phagocytosis [333]. In this study, CD11b expression increased significantly but not dose-dependently in response to all three TLR agonists. Under all TLR stimulation conditions, CD11b expression increased by 63-93\% from unstimulated cultures. These results suggest that $C D 11 \mathrm{~b}$ is upregulated on the neutrophil surface, perhaps to a maximum 
level, with $\geq 1 \mathrm{ng} / \mathrm{mL}$ TLR stimulation. It may be that CD11b expression is sensitive to stimulation, and may rapidly upregulate or reach saturation in response to broad TLR stimulation. Despite a uniform increase in CD11b expression with TLR stimulation, this expression was only sensitive to the effect of platelets in response to Pam3CSK4 and FSL-1. It may be that the microenvironment following LPS stimulation restricts the ability of platelets to have the same dampening effect seen in response to the other TLR agonists.

Similarly to the results seen with CD66b expression, platelets decreased CD11b expression by 10$13 \%$ in response to Pam3CSK4 and FSL-1, regardless of dosage. Furthermore, platelets did not alter neutrophil CD11b expression in unstimulated cultures or in response to LPS. CD11b expression was examined in this study to determine whether, in our model of human neutrophilplatelet co-cultures, platelets had the same dampening effect on CD11b expression as was observed by Corken and colleagues in a mouse model of polymicrobial sepsis [18]. As discussed above, Corken et al. demonstrated that the loss of a single platelet receptor, GP Ib-IX, was sufficient to increase the level of neutrophil CD11b expression that occurs 24 hours post the onset of sepsis in mice [18]. The authors conclude that, through the action of GP Ib-IX, platelets were able to dampen neutrophil CD11b expression in response to systemic inflammation, which is characterized by high exposure to TLR-triggering stimuli. We also confirm that platelets can reduce CD11b expression in response to particular TLR stimuli and that this platelet effect is not seen in unstimulated cultures. Considering the results from Corken, et al. it becomes of interest to determine whether platelets can reduce neutrophil CD11b expression when stimulated with a combination of TLR agonists in our human neutrophil-platelet culture model, as a combination of these agonists may more accurately mimic systemic inflammation.

CD62L shedding was also examined here as another marker of neutrophil activation. CD62L shedding is known to occur following TLR stimulation [254] and we also demonstrate a significant dose-dependent reduction in CD62L expression in response to all three TLR agonists tested in this study. Interestingly, platelets attenuated the level of CD62L shedding (increased CD62L 
expression) in unstimulated cultures, demonstrating that platelets can exert an effect on some markers of neutrophil activation in the absence of TLR stimulation. Conversely, both CD66b and CD11b expression levels were unaltered in the presence of platelets in unstimulated cultures. CD62L shedding was also attenuated in response to high-dose Pam3CSK4 and low-dose FSL-1, but was not attenuated in response to LPS. We suggest that platelets can regulate the expression of CD62L without stimulation, and the platelet effect seen in unstimulated cultures can be overcome with LPS stimulation but can be maintained with particular doses of Pam3CSK4 and FSL-1. All three flow cytometric markers of neutrophil activation measured in this study were more sensitive to modulation by platelets in response to Pam3CSK4 and FSL-1, and the levels of these markers were not modulated at all by platelets following LPS stimulation.

Within this study, we also assessed the effect of platelets on elastase and IL-8 secretion by neutrophils in response to TLR agonism. In this study, we shown that the addition of platelets significantly attenuated elastase secretion in unstimulated cultures and in response to low-dose TLR agonism. Elastase secretion was also measured in Chapter 5 and, consistent with this study's findings, was shown to be significantly attenuated by low-dose TLR stimulation and nonsignificantly attenuated in unstimulated cultures. A previous study has also shown that the presence of platelets can reduce elastase secretion [308]. Neutrophil elastase is a potent serine protease which can degrade a multitude of extracellular matrix and plasma proteins. As such, extracellular elastase is a powerful bacterial killing mechanism [334] but has also been implicated in acute and chronic inflammatory host damage [335, 336]. Platelets may dampen elastase secretion as a mechanism to prevent inflammatory host damage. From this study's results, we can conclude that platelets have a significant dampening effect on neutrophil elastase secretion in the absence of stimulation and the effect is consistently maintained with low-dose TLR stimulation. Interestingly, IL-8 secretion was only affected by platelets in response to low-dose LPS stimulation: IL-8 secretion was further increased by $28 \%$ under this stimulation condition. IL- 8 is known to be produced by neutrophils in response to TLR stimulation [254], and is an important proinflammatory neutrophil chemoattractant protein [337]. It may be that, in particular stimulation conditions, platelets can increase neutrophil IL-8 secretion and indirectly increase neutrophil 
chemoattraction to the site of infectious or injurious stimuli. However, it is unclear why an effect of platelets was not observed under any other condition tested. Examining a larger panel of cytokines and chemokines secreted by neutrophils is required to contextualize the significance of the platelet effect on IL-8 secretion noted in this study.

Phagocytosis is a crucial neutrophil function for effective host defence, and the effect of platelets on the phagocytic activity of neutrophils was assessed in this study. At baseline, we show that phagocytosis significantly increased in response to all three TLR agonists. In particular, there was a uniform increase in phagocytic activity (approximately $70 \%$ of the neutrophil population was FITC-positive) in response to high doses of all TLR agonists as well as in response to low-dose LPS. In response to low-dose Pam3CSK4 and FSL-1, phagocytic rates were also very similar (41\% of FITC+ neutrophils). It is possible that phagocytosis reaches a maximum at high-dose TLR stimulation. While lower rates of phagocytosis can be seen following low-dose Pam3CSK4 and FSL1 stimulation, this maximum rate can still be achieved following low-dose LPS stimulation. The addition of platelets substantially increased phagocytosis in unstimulated cultures (121\% increase) and in response to low-dose stimulation with Pam3CSK4 and FSL-1 (35\% and 31\% increase). For each of the other condition tested, the high (perhaps maximum) phagocytic activity that was recorded is likely to limit the ability of platelets to further enhance this activity. It may be that platelets have an effect on phagocytic activity under these stimulation conditions within a shorter incubation time, which we were unable to capture here. Our results are corroborated by others: one study demonstrated that phagocytosis of periodontopathogens by neutrophils is enhanced by platelets [192], and another demonstrated that neutrophils within platelet-neutrophil complexes had increased phagocytic activity when compared to neutrophils that did not bind to platelets [308].

Interestingly, the effect of platelets on neutrophil phagocytic activity and IL-8 secretion was in opposition to the effect of platelets on elastase secretion and on the expression of activation marker seen in this study. The combination of the platelet effects described in this study suggest 
that platelets can both augment and attenuate neutrophil functions, and modulation of these functions can occur in both the absence and presence of TLR stimulation. For example, we showed that platelets can simultaneously reduce elastase secretion and CD62L shedding while increasing phagocytic activity in unstimulated cultures. Similarly, in response to low-dose FSL-1 stimulation, platelets attenuated neutrophil activation (as evidenced by all three flow cytometric activation markers) and attenuated elastase secretion but increased phagocytosis. The opposing effect of platelets noted here may be a mechanism for enhancing particular anti-microbial functions (for example, phagocytosis) while dampening neutrophil functions that are more likely to lead to host damage, if unchecked (for example, elastase secretion). Overall, we suggest that platelets finely regulate neutrophil functions, rather than providing broad anti-inflammatory cues to these cells, and we postulate that the platelet effect can modulate some aspects of the inflammatory environment to reduce host damage.

As with the previous study (Chapter 5 ), this study examined the platelet effect on neutrophil responses to TLR stimulation in healthy subjects. As the platelet effect has not been well-defined in this context previously, it was important to establish the extent to which platelets can modulate neutrophil function in healthy subjects prior to conducting this study in diseased states such as AMI. However, it is interesting to hypothesise how the results from this study can be interpreted in the context of an AMI, which is known to induce intense sterile inflammation. Emerging research supports the notion that platelets can dampen leukocyte-mediated pro-inflammatory responses during excessive inflammation [22], and it can be postulated that platelets perform this role during an AMI. It is also interesting to postulate the effect of anti-platelet therapy on these platelet effects. Although necessary to inhibit the pro-thrombotic effects of platelets during AMI, this treatment strategy may inhibit other, more subtle and perhaps beneficial, platelet immune effects. The effect of platelets on leukocyte function during AMI is interesting research that remains unexplored. 
We hypothesize that the complex and intense cross-talk between platelets and neutrophils drives the platelet effect that we have seen in this Chapter. Neutrophils become strongly activated in response to the dose range of TLR agonists used here, and we suggest that this activation is likely to drive a number of platelet responses. This is particularly in light of the knowledge that platelets do not become directly activated to all of these TLR agonists, as demonstrated in previously chapters. Thus, a lack of direct platelet activation by these agonists is compensated by an indirect effect of TLR agonist-stimulated neutrophils. If this is the case, the complexity of the cross-talk becomes evident here: neutrophils are able to become activated in response to TLR stimulation and are further able to mediate platelet responses, and platelets, in turn, act to dampen neutrophil activation. It is important to recognize that the dampening platelet effect is a result of very complex, nuanced and subtle cross-talk between these cell types. Although not elucidated here, the exact interactions between these cells that elicits this dampening response is important to understand and ought to be the subject of future investigations.

It is of interest to determine the extent to which the platelet effects noted in this study are reproducible in vivo. For example, it may be that markers of neutrophil function inversely correlate with platelet count. This would allow us to determine the physiological relevance of the platelet effect seen here, and provide an in vivo model for examining the effect of infection, AMI or antiplatelet medication on these correlations. This is the subject of ongoing investigation by our laboratory group. 


\subsection{Limitations.}

In vitro assessment of neutrophil function required isolation of neutrophils from whole blood in order to standardize cell counts. This may have altered the activation state of neutrophils in unstimulated cultures and may have acted as a neutrophil priming step. However, we ensured that neutrophils were isolated with a minimum number of steps and under conditions to minimize this type of activation. In isolating the neutrophil and platelet fractions from blood, we excluded the effect of any other factor present within the blood. These cells do not act in isolation and it is likely that, within whole blood, the modulation of neutrophil function by platelets differs. However, the experimental methodology was appropriate to assess the aim of this study, which was to determine the effect of platelets on neutrophil responses to TLR stimulation. Assessing how the platelet effect on neutrophils changes in whole blood is currently being undertaken by our laboratory group. As with the previous study, we did not assess whether the platelet effect was due to direct interaction of platelets with neutrophils or due to the effect of platelet-mediated secretory products. The literature suggests that both interactions contribute to this platelet effect, and we were interested in capturing both the direct and indirect effects of platelets in this study. It is possible that platelets may exert some effect on neutrophils following stimulation with $<1$ $\mathrm{ng} / \mathrm{mL}$ of the TLR agonists used in this study, which we were unable to capture. However, due to the high volume of experimental work and in order to ensure reproducibility and consistency, we chose to examine only two doses of each TLR agonist.

Page / 229 


\subsection{Conclusion.}

In conclusion, platelets can modify a number of neutrophil functions in both the absence of stimulation and in response to TLR stimulation. Furthermore, platelets were shown to attenuate some, and simultaneously augment other, neutrophil functions. We suggest that platelets can finely regulate neutrophil function, rather than provide consistent dampening cues, and that platelets are able to modulate some aspects of neutrophil-mediated inflammation to reduce host damage. 
7 Chapter 7. Summary and future directions. 


\subsection{Summary of studies.}

The aims of the study outlined in Chapter 2 were to examine baseline TLR9 expression in healthy subjects and in AMI subjects treated with DAPT, to determine how TLR9 expression changed in response to platelet activation in these cohorts and to examine platelet activation in response to ODN2006, a TLR9 agonist, in these cohorts. We show that baseline TLR9 expression was significantly greater in AMI subjects compared to healthy subjects and, following activation with TRAP (a traditional platelet agonist), TLR9 expression increased dose-dependently in healthy subjects. However, no difference in TLR9 expression was seen in AMI platelets following TRAP activation. ODN2006 treatment resulted in significant increases in cell-surface expression of CD62p and CD63 (well-characterized platelet activation markers) in both WB and PRP in comparison to unstimulated platelets in healthy subjects. Despite DAPT, ODN2006 treatment produced significant increases in both activation markers in the AMI cohort across both WB and PRP. Elevated baseline expression of TLR9 in AMI platelets may indicate increased sensitivity to TLR9 agonists and contribute to increased platelet activation in these patients. Furthermore, ODN2006 stimulation can activate platelets in AMI subjects despite treatment with DAPT. This study demonstrates TLR9 expression and activation to be of potential therapeutic importance in AMI patients.

For Chapter 3, we aimed to examine whether total protein expression of platelet-TLR1, 2, 4 and 6 changed between healthy subjects and AMI subjects treated with DAPT, and to compare the level of platelet activation in response to TLR4, TLR2/1 and TLR2/6 agonism in both of these cohorts. Here, we report that platelets from AMI patients exhibit upregulation of some, but not other, TLRs. Platelet-TLR1 and TLR4 were significantly upregulated in AMI subjects compared to healthy subjects. Platelet-TLR2 and platelet-TLR6 expression did not significantly change across cohorts. Platelets from both healthy and AMI subjects exhibited distinct activation patterns in response to various TLR agonists $(0.1-100 \mu \mathrm{g} / \mathrm{mL})$. Healthy and AMI platelets became dose-dependently and directly activated in response to Pam3CSK4, a TLR2/1 agonist, but were directly potently activated 
only in response to the highest dose $(100 \mu \mathrm{g} / \mathrm{mL})$ of LPS, a TLR4 agonist. This was evidenced by increases in expression of both CD62p and PAC1 (well-known activation markers). Platelet activation in response to these TLR agonists was similar across cohorts, despite treatment with anti-platelet therapy in the AMI cohort. At all doses used in this study, platelets were unable to become directly activated by FSL-1, a TLR2/6 agonist. We also show that PMAgg occurs in a dosedependent manner in response to TLR2/1 stimulation, but PMAgg was not significantly elevated following LPS stimulation and only elevated following high-dose FSL-1 stimulation in healthy subjects. We conclude that the platelet-TLR2/1 activation pathway is functional post-AMI, despite treatment with anti-platelet therapy. The platelet-TLR4 pathway appears to be less likely, and the platelet-TLR2/6 pathways unlikely, to contribute to post-AMI platelet activation.

In Chapters 2 and 3, we were unable to assess TLR-mediated platelet activation in the absence of anti-platelet therapy in patients with $\mathrm{AMI}$ as these drugs are administered prior to or immediately following presentation to hospital. Consequently, we could not establish the extent to which DAPT provides protection against TLR-mediated platelet activation. This was addressed in Chapter 4: we aimed to determine the extent to which aspirin alone or DAPT (aspirin and ticagrelor) can inhibit platelet activation in response to TLR2/1, TLR4 and TLR9 stimulation. We conducted a singleblinded randomized cross-over trial, with subjects receiving aspirin monotherapy and DAPT (aspirin in combination with ticagrelor) over the course of the study. We showed that, at baseline, expression of CD62p and PAC1 increased significantly in response to high-dose LPS and in a dosedependent manner in response to Pam3CSK4 and ODN2006. Aspirin monotherapy did not inhibit platelet activation in response to any TLR agonist tested. DAPT with aspirin and ticagrelor only modestly inhibited expression of both activation markers in response to high doses of Pam3CSK4 and LPS and in response to multiple doses of ODN2006. Incubation with these TLR agonists led to substantial platelet activation despite treatment with anti-platelet agents. We suggest that these platelet-TLRs represent intact on-treatment platelet activation pathways, which may contribute to ongoing platelet activation post-AMI and despite anti-platelet therapy. 
In Chapter 3, we did not fully characterize the inflammatory responses of platelets beyond assessing PMAgg in response to TLR agonism. Therefore, in Chapter 5, we aimed to determine the extent to which platelets modulate PBMC (monocyte, T cell) and granulocyte (neutrophil) responses in vitro to TLR4, TLR2/1, and TLR2/6 agonism in healthy subjects. Platelet co-culture significantly decreased neutrophil CD66b expression (a marker of neutrophil activation) in response to LPS, Pam3CSK4 and FSL-1. With platelets, monocyte HLA-DR expression (monocyte activation marker) in response to high-dose LPS was modestly attenuated while CD8+ T-cell CD38 expression (T cell activation marker) in response to low-dose Pam3CSK4 and high-dose FSL-1 was modestly increased. Platelets markedly attenuated granulocyte elastase production in response to low-doses of all TLR agonists. In platelet co-cultures in response to LPS agonism, there was a significant reduction in IL-6 and TNF- $\alpha$ production, and increased IL-10 production by PBMCs. In response to FSL-1 stimulation, platelet co-culture increased IL-6 and IL-10 production, but attenuated TNF- $\alpha$ production. Platelet co-culture did not alter PBMC cytokine production in response to Pam3CSK4. In combination, these results show that platelets are complex immunomodulators, and the platelet effect on leukocyte function differs depending on the leukocyte subset and the TLR agonist used.

Due to the broad nature of the study outlined in Chapter 5, we did not characterize the platelet effect on neutrophil function beyond the measurement of CD66b expression and elastase secretion. Therefore, in Chapter 6, we aimed to determine the extent to which platelets can modulate multiple markers of neutrophil function in response to in vitro stimulation with TLR4, TLR2/1, and TLR2/6 agonists in healthy subjects. We showed that neutrophil CD66b and CD11b expression was increased in response to various doses of Pam3CSK4 and FSL-1, and expression of both markers was significantly attenuated in the presence of platelets. Platelets did not alter CD66b or CD11b expression in response to LPS. Also, in unstimulated cultures and following stimulation with high-dose Pam3CSK4 and low-dose FSL-1, CD62L expression was significantly increased in the presence of platelets. Elastase production was attenuated in the presence of platelets in unstimulated cultures as well as in response to low-dose stimulation with all three TLR 
agonists. We also showed that the increase in IL-8 production in response to low-dose LPS was further increased in the presence of platelets. Furthermore, in the presence of platelets, the phagocytic activity of neutrophils was increased in unstimulated cultures and in response to lowdose Pam3CSK4 and FSL-1. In conclusion, platelets modify a number of neutrophil functions in both the absence of stimulation and in response to TLR stimulation. Platelets were able to attenuate some, but not other, neutrophil functions, and we suggest that platelets act as regulators of the neutrophil response to infection and inflammation. 


\subsection{Limitations.}

There have been some overarching limitations throughout this thesis that may have influenced our findings, and which must be acknowledged.

One particular limitation is that, throughout these studies, we have examined TLR-mediated platelet activation or the platelet effect on leukocytes in an in vitro setting. The experimental design for each of these studies was appropriate, as we aimed to examine platelet-TLR effects in human cells and, further, to examine the direct effects (rather than the effects within whole blood, for example) of either the TLR agonists acting on platelets or of platelets acting on leukocytes. We understand that these in vitro results may not fully or accurately predict these effects within whole blood and that extrapolation of these effects into in vivo human studies is required. However, the results reported in this thesis allow us to determine the direction of future in vivo studies.

We designed many of the studies within this thesis around the availability of commercially available and quality tested TLR agonists and antibodies, as it was our aim to assess platelet and leukocyte responses to TLR stimulation using appropriate pre-existing tools. As such, we did not validate the specificity of these agonists or antibodies. We, therefore, cannot rule out that these agonists may be acting on platelets and leukocytes via other non-TLR pathways or that these antibodies may recognize off-target epitopes. However, each was thoroughly validated by the providing companies and was highly reproducible between our studies. Also, each antibody used here had an expected pattern of expression, based on expression data in the literature.

For each of the TLR agonists used in this thesis, it is unknown what would constitute a physiologically or clinically relevant dose that would be present during AMI or sterile inflammation. It is possible that high localized concentrations of TLR agonists exist in the myocardium or at the ruptured plaque site with lower concentrations of these agonists circulating systemically. Due to this unknown, the doses of the commercially available agonists used here were based on previous 
studies examining platelet activation and leukocyte culturing and we conducted dose-response curves for each of these agonists to determine appropriate doses to use.

In the setting of AMI, it is likely that DAMPs are a source of platelet activation via their TLRs. However, we do not know the extent to which TLR-triggering DAMPs are present during AMI. We also do not know what sort of affinity that these DAMPs have for each of these TLRs. For example, activation may require high concentrations of a DAMP with a low-affinity for a TLR and, therefore, the presence of this DAMP may not contribute to in vivo platelet activation post-AMI. The use of quality-tested, commercially available TLR agonists has allowed us to establish the functionality of platelet-TLRs, although further investigation is warranted into how these platelet responses change when exposed to DAMPs during AMI.

For Chapters 2 and 3, we recruited relatively small, real-world population of AMI patients to analyse platelet-TLR expression and TLR-mediated platelet activation. Recruitment of larger cohorts would have allowed for greater statistical power to perform sub-group analysis. For example, NSTEMI and STEMI present with different extents of myocardial injury and, as a result, may have different platelet-TLR expression levels and platelet responses to TLR stimulation. Similarly, anti-platelet agents (clopidogrel vs. ticagrelor) differ in their effects on platelet inhibition and may have different effects on TLR-mediated platelet activation. However, the aims of these studies were to describe platelet-TLR expression and TLR-mediated platelet activation at a cohort level, and were not designed to answer these questions. 


\subsection{Future directions.}

The findings from this thesis indicate the potential clinical importance of certain platelet-TLR pathways in the setting of $\mathrm{AMI}$ and raise avenues for future research.

TLR signalling in nucleated cells involves the nuclear translocation of transcription factors and, fundamentally, this signalling pathway must be different in anucleate platelets. It is possible that the TLR signalling pathway within platelets feeds into traditional platelet activation pathways, although the exact pathway by which TLRs trigger platelet activation in platelets is currently unknown. Determining the point at which the TLR signalling pathways and platelet activation pathways interact may identify potential therapeutic targets for reducing TLR-mediated platelet activation.

In Chapter 4, we showed that platelets continue to become activated by TLR stimulation despite DAPT. It is important, therefore, to identify whether any clinically available drug can be added to DAPT to more effectively inhibit TLR-mediated platelet activation. Candesartan is an angiotensin II receptor antagonist prescribed for high blood pressure and can be prescribed to AMI patients, alongside DAPT. Interestingly, other groups have shown that candesartan can inhibit TLR signalling in other blood cell types [338-340]. It is of interest to determine whether candesartan can also inhibit TLR signalling in platelets and, thereby, reduce platelet activation in response to TLR agonism. Using a cross-over study design similar to that described in Chapter 4, we could determine the extent to which treatment with candesartan, with and without DAPT, can inhibit TLR-mediated platelet activation. If candesartan can provide a greater level of protection against TLR-mediated platelet activation than DAPT alone, the clinical utility of this combined drug regimen could be tested. 
In Chapters 5 and 6, we investigated the effects of platelets on leukocyte response to TLR stimulation in an in vitro setting. However, it is important to determine how the platelet effect on leukocytes differs within a whole blood system. For example, it is of interest to determine the correlation between platelet count/activation and neutrophil activation/function in whole blood, and how well this correlation reflects the results we observed in Chapter 6. This would give us a better understanding of the effects of platelets in a physiologically relevant setting and would establish a methodology to determine whether this correlation is altered over the course of an AMI or with administration of anti-platelet agents.

The results from Chapters 5 and 6 indicated that platelets can differentially regulate leukocyte responses to TLR agonism, and the effect of platelets can both attenuate and augment these leukocyte responses. There are a number of different experimental designs that can investigate these platelet effects further. Firstly, we did not assess whether leukocyte responses were affected by direct platelet interaction or by the indirect release of platelet modulators, although the literature suggests that platelets act on leukocytes via both direct and indirect mechanisms. The first step for examining these results further, therefore, is to determine how these platelet effects are altered if cell-cell contact is inhibited. Secondly, the design of this study meant that platelets could become activated via direct TLR stimulation and via indirect activation by leukocytes within cultures. It would be interesting to determine how much of the platelet effect that we saw in Chapters 5 and 6 was mediated directly by platelet-TLR stimulation and this could be achieved with pre-incubation of platelets with neutralizing antibodies to these TLRs prior to culturing. Thirdly, one study has demonstrated that a number of these platelet effects can be mediated by a single platelet glycoprotein receptor [18]. It would also be interesting, therefore, to determine which pathways are crucial, and which ones are redundant, to these platelet effects.

In considering the results from Chapter 6, it would be interesting to determine how these platelet effects are altered following anti-platelet therapy. This is of particular importance considering the sterile inflammatory nature of AMI and the use of anti-platelet therapy in these patients. For 
example, we suggest that platelets act as a brake to ensure that inflammation is regulated, but we do not yet know whether anti-platelet therapy abolishes these potentially protective effects. Although necessary for reducing platelet activation, anti-platelet therapy may prevent these moderate immunomodulatory effects. Using a similar study design as described in Chapter 4, we could determine the extent to which treatment with DAPT can alter these platelet-neutrophil interactions.

For all of these studies, platelet activation or the platelet effect on leukocytes has been characterised following activation with a single TLR agonist. It is also of interest to determine how these patterns change when exposed to a multitude of TLR agonists, which may better reflect the inflammatory milieu during AMI or sterile inflammation. Aside from the use of specific TLR agonists, it is interesting to determine how platelets become activated in response to DAMPs such as HMGB1 or S100 proteins, both of which are released during AMI [341] and which can signal via both TLR-dependent and TLR-independent pathways. Investigating the platelet effect in response to DAMPs is highly clinically relevant, as a larger area of myocardial ischaemia and infarction is likely to trigger a larger release of DAMPs and a correspondingly larger inflammatory response post-AMI. Elucidating the platelet effect within this system is crucial to determining whether the development of agents to suppress these TLR pathways is worthwhile. 


\subsection{Conclusion.}

In this thesis, we aimed to characterize the importance of the platelet-TLR pathways in healthy and AMI subjects. We demonstrated that expression of a number of TLRs (TLRs, 1, 4, 9) was increased in AMI subjects, when compared to healthy subjects. We also showed that platelets can become directly activated by some (TLRs $2 / 1,4$, and 9), but not other (TLR2/6), TLR agonists. These patterns of platelet activation were similar between healthy and AMI subjects, and this activation occurred despite treatment with DAPT in the AMI cohort. In healthy subjects, we further demonstrated that DAPT (aspirin and ticagrelor) only moderately, and aspirin monotherapy did not, inhibit platelet activation in response to TLR2/1, TLR4 and TLR9 agonism. We conclude that these platelet-TLR pathways represent alternative platelet activation pathways that remain intact despite treatment with current anti-platelet agents and may contribute to persistent platelet activation post-AMI. We also showed that platelets are able to modulate leukocyte responses to TLR stimulation in a TLR agonist-specific and leukocyte subset-specific manner. When investigated further, we determined that platelets can modulate multiple markers of neutrophil function in unstimulated cultures and in response to TLR stimulation, and that platelets were able to augment some markers and simultaneously attenuate other markers. We conclude that platelets regulate leukocyte responses to inflammatory stimuli. This may be a mechanism to prevent host damage during excessive inflammation, such as in the context of an AMI. Overall, we have demonstrated that some, but not all, platelet-TLRs are important in eliciting a number of thrombotic and inflammatory responses in healthy subjects and in subjects with AMI.

Page / 241 


\section{References.}

1. Jennings, L.K., Mechanisms of platelet activation: need for new strategies to protect against platelet-mediated atherothrombosis. Thromb Haemost, 2009. 102(2): p. 248-57.

2. Bennett, J.S., Structure and function of the platelet integrin alphallbbeta3. Journal of Clinical Investigation, 2005. 115(12): p. 3363-9.

3. Wagner, C., et al., Analysis of GPIIb/IIla receptor number by quantification of $7 E 3$ binding to human platelets. Blood, 1996. 88(3): p. 907-914.

4. Bennett, J.S. and G. Vilaire, Exposure of platelet fibrinogen receptors by ADP and epinephrine. Journal of Clinical Investigation, 1979. 64(5): p. 1393-401.

5. Fullard, J.F., The role of the platelet glycoprotein IIb/IIla in thrombosis and haemostasis. Curr Pharm Des, 2004. 10(14): p. 1567-76.

6. Hartwig, J.H., Mechanisms of actin rearrangements mediating platelet activation. Journal of Cell Biology, 1992. 118(6): p. 1421-42.

7. Martorell, L., et al., Thrombin and protease-activated receptors (PARs) in atherothrombosis. Thromb Haemost, 2008. 99(2): p. 305-15.

8. Yeaman, M.R., Platelets: at the nexus of antimicrobial defence. Nature Reviews Microbiology, 2014. 12(6): p. 426-437.

9. Tang, Y.Q., M.R. Yeaman, and M.E. Selsted, Antimicrobial peptides from human platelets. Infection and Immunity, 2002. 70(12): p. 6524-33.

10. Youssefian, T., et al., Host defense role of platelets: engulfment of HIV and Staphylococcus aureus occurs in a specific subcellular compartment and is enhanced by platelet activation. Blood, 2002. 99(11): p. 4021-9.

11. Riaz, A.H., et al., Human platelets efficiently kill IgG-opsonized E. coli. Fems Immunology and Medical Microbiology, 2012. 65(1): p. 78-83.

12. Lee, K.H., K.P. Hui, and W.C. Tan, Thrombocytopenia in sepsis: a predictor of mortality in the intensive care unit. Singapore Med J, 1993. 34(3): p. 245-6.

13. Xiang, B., et al., Platelets protect from septic shock by inhibiting macrophage-dependent inflammation via the cyclooxygenase 1 signalling pathway. Nature Communications, 2013. 4: p. 2657.

14. Wuescher, L.M., A. Takashima, and R.G. Worth, A novel conditional platelet depletion mouse model reveals the importance of platelets in protection against Staphylococcus aureus bacteremia. Journal of Thrombosis and Haemostasis, 2015. 13(2): p. 303-13.

15. de Stoppelaar, S.F., et al., Thrombocytopenia impairs host defense in gram-negative pneumonia-derived sepsis in mice. Blood, 2014. 124(25): p. 3781-90.

16. Hechler, B., et al., A Potential Protective Role of Platelets during Septic Shock Does Not Depend on Their Purinergic Receptors. Blood, 2016. 128(22): p. 2537-2537.

17. Bacci, M.R., et al., IL-6 and TNF- $\alpha$ serum levels are associated with early death in community-acquired pneumonia patients. Brazilian Journal of Medical and Biological Research, 2015. 48(5): p. 427-432.

18. Corken, A., et al., Platelet glycoprotein $I b$-IX as a regulator of systemic inflammation. Arterioscler Thromb Vasc Biol, 2014. 34(5): p. 996-1001. 
References.

19. Clark, S.R., et al., Platelet TLR4 activates neutrophil extracellular traps to ensnare bacteria in septic blood. Nature Medicine, 2007. 13(4): p. 463-469.

20. Verschoor, A., et al., A platelet-mediated system for shuttling blood-borne bacteria to CD8[alpha]+ dendritic cells depends on glycoprotein GPIb and complement C3. Nature Immunology, 2011. 12(12): p. 1194-1201.

21. Stokes, K.Y. and D.N. Granger, Platelets: a critical link between inflammation and microvascular dysfunction. The Journal of Physiology, 2012. 590(Pt 5): p. 1023-1034.

22. Stocker, T.J., et al., Small but mighty: Platelets as central effectors of host defense. Thromb Haemost, 2017. 117(4): p. 651-661.

23. Gudbrandsdottir, S., H.C. Hasselbalch, and C.H. Nielsen, Activated platelets enhance IL-10 secretion and reduce TNF-alpha secretion by monocytes. Journal of Immunology, 2013. 191(8): p. 4059-67.

24. Nami, N., et al., Crosstalk between platelets and PBMC: New evidence in wound healing. Platelets, 2016. 27(2): p. 143-8.

25. Linke, B., et al., Activated Platelets Induce an Anti-Inflammatory Response of Monocytes/Macrophages through Cross-Regulation of PGE2 and Cytokines. Mediators Inflamm, 2017. 2017: p. 1463216.

26. Sadallah, S., et al., Ectosomes released by platelets induce differentiation of CD4+T cells into T regulatory cells. Thromb Haemost, 2014. 112(6): p. 1219-29.

27. Sadallah, S., et al., Microparticles (ectosomes) shed by stored human platelets downregulate macrophages and modify the development of dendritic cells. Journal of Immunology, 2011. 186(11): p. 6543-52.

28. Jancinova, V., et al., Blood platelets decrease concentration of reactive oxygen species produced by polymorphonuclear leukocytes. Bratisl Lek Listy, 2004. 105(7-8): p. 250-5.

29. Del Principe, D., et al., Stimulated platelets release factor(s) affecting the in vitro response of human polymorphonuclear cells. Journal of Leukocyte Biology, 1990. 48(1): p. 7-14.

30. Milioli, M., et al., Quantitative proteomics analysis of platelet-derived microparticles reveals distinct protein signatures when stimulated by different physiological agonists. Journal of Proteomics, 2015. 121: p. 56-66.

31. Vélez, P., et al., A 2D-DIGE-based proteomic analysis reveals differences in the platelet releasate composition when comparing thrombin and collagen stimulations. Scientific Reports, 2015. 5: p. 8198.

32. Chatterjee, M., et al., Distinct platelet packaging, release, and surface expression of proangiogenic and antiangiogenic factors on different platelet stimuli. Blood, 2011. 117(14): p. 3907-11.

33. Pokrovskaya, I.D., et al., STEM tomography reveals that the canalicular system and alphagranules remain separate compartments during early secretion stages in blood platelets. Journal of Thrombosis and Haemostasis, 2016. 14(3): p. 572-84.

34. Health, M.o., Mortality and Demographic data. 2015.

35. Ellis, C., et al., Patients admitted with an acute coronary syndrome (ACS) in New Zealand in 2007: results of a second comprehensive nation wide audit and a comparison with the first audit from 2002. New Zealand Medical Journal, 2010. 123(1319): p. 25-43.

36. Stary, H.C., et al., A Definition of Advanced Types of Atherosclerotic Lesions and a Histological Classification of Atherosclerosis. A Report From the Committee on Vascular 
Lesions of the Council on Arteriosclerosis, American Heart Association, 1995. 92(5): p. 1355-1374.

37. Berenson, G.S., et al., Association between multiple cardiovascular risk factors and atherosclerosis in children and young adults. The Bogalusa Heart Study. New England Journal of Medicine, 1998. 338(23): p. 1650-6.

38. Nakashima, Y., et al., Upregulation of VCAM-1 and ICAM-1 at atherosclerosis-prone sites on the endothelium in the ApoE-deficient mouse. Arterioscler Thromb Vasc Biol, 1998. 18(5): p. 842-51.

39. Vion, A.C., et al., Shear stress regulates endothelial microparticle release. Circulation Research, 2013. 112(10): p. 1323-33.

40. Leitinger, N., Oxidized phospholipids as modulators of inflammation in atherosclerosis. Current Opinion in Lipidology, 2003. 14(5): p. 421-30.

41. Massberg, S., et al., A critical role of platelet adhesion in the initiation of atherosclerotic lesion formation. Journal of Experimental Medicine, 2002. 196(7): p. 887-96.

42. Jonasson, L., et al., Regional accumulations of T cells, macrophages, and smooth muscle cells in the human atherosclerotic plaque. Arteriosclerosis, 1986. 6(2): p. 131-8.

43. Kovanen, P.T., M. Kaartinen, and T. Paavonen, Infiltrates of activated mast cells at the site of coronary atheromatous erosion or rupture in myocardial infarction. Circulation, 1995. 92(5): p. 1084-8.

44. Fok, P.W., Growth of necrotic cores in atherosclerotic plaque. Math Med Biol, 2012. 29(4): p. 301-27.

45. Ross, R., Atherosclerosis--an inflammatory disease. New England Journal of Medicine, 1999. 340(2): p. 115-26.

46. Glagov, S., et al., Compensatory enlargement of human atherosclerotic coronary arteries. New England Journal of Medicine, 1987. 316(22): p. 1371-5.

47. Skeoch, S. and I.N. Bruce, Atherosclerosis in rheumatoid arthritis: is it all about inflammation? Nature Reviews Rheumatology, 2015. 11(7): p. 390-400.

48. Fuster, V., et al., The Pathogenesis of Coronary Artery Disease and the Acute Coronary Syndromes. New England Journal of Medicine, 1992. 326(4): p. 242-250.

49. Chew, D.P., et al., National Heart Foundation of Australia \& Cardiac Society of Australia and New Zealand: Australian Clinical Guidelines for the Management of Acute Coronary Syndromes 2016. Heart Lung Circ, 2016. 25(9): p. 895-951.

50. Boateng, S. and T. Sanborn, Acute myocardial infarction. Disease-a-Month, 2013. 59(3): p. 83-96.

51. Davies, M.J., Stability and Instability: Two Faces of Coronary Atherosclerosis. The Paul Dudley White Lecture 1995, 1996. 94(8): p. 2013-2020.

52. Hackett, D., G. Davies, and A. Maseri, Pre-existing coronary stenoses in patients with first myocardial infarction are not necessarily severe. European Heart Journal, 1988. 9(12): p. 1317-1323.

53. Yonetsu, T., et al., In vivo critical fibrous cap thickness for rupture-prone coronary plaques assessed by optical coherence tomography. European Heart Journal, 2011. 32(10): p. 1251-1259. 
References.

54. Deguchi, J.O., et al., Matrix metalloproteinase-13/collagenase-3 deletion promotes collagen accumulation and organization in mouse atherosclerotic plaques. Circulation, 2005. 112(17): p. 2708-15.

55. Newby, A.C., Metalloproteinase Expression in Monocytes and Macrophages and its Relationship to Atherosclerotic Plaque Instability. Arteriosclerosis, Thrombosis, and Vascular Biology, 2008. 28(12): p. 2108-2114.

56. Finn, A.V., et al., Concept of vulnerable/unstable plaque. Arterioscler Thromb Vasc Biol, 2010. 30(7): p. 1282-92.

57. Davies, M.J., The contribution of thrombosis to the clinical expression of coronary atherosclerosis. Thromb Res, 1996. 82(1): p. 1-32.

58. Bentzon, J.F., et al., Mechanisms of Plaque Formation and Rupture. Circulation Research, 2014. 114(12): p. 1852-1866.

59. Nagel, T., et al., Shear stress selectively upregulates intercellular adhesion molecule-1 expression in cultured human vascular endothelial cells. Journal of Clinical Investigation, 1994. 94(2): p. 885-91.

60. Andre, P., et al., Platelets adhere to and translocate on von Willebrand factor presented by endothelium in stimulated veins. Blood, 2000. 96(10): p. 3322-8.

61. Platelets. 2002: Academic Press.

62. Freedman, J.E., et al., Decreased platelet inhibition by nitric oxide in two brothers with a history of arterial thrombosis. Journal of Clinical Investigation, 1996. 97(4): p. 979-987.

63. Ware, J.A. and D.D. Heistad Platelet-Endothelium Interactions. New England Journal of Medicine, 1993. 328(9): p. 628-635.

64. Silvain, J., et al., Composition of coronary thrombus in acute myocardial infarction. Journal of the American College of Cardiology, 2011. 57(12): p. 1359-67.

65. Hansson, G.K. and P. Libby, The immune response in atherosclerosis: a double-edged sword. Nat Rev Immunol, 2006. 6(7): p. 508-519.

66. Bigalke, B., et al., Expression of platelet collagen receptor glycoprotein $\mathrm{VI}$ is associated with acute coronary syndrome. European Heart Journal, 2006. 27(18): p. 2165-9.

67. Wang, Y., et al., Leukocyte engagement of platelet glycoprotein Ibalpha via the integrin Mac-1 is critical for the biological response to vascular injury. Circulation, 2005. 112(19): p. 2993-3000.

68. Bultmann, A., et al., Impact of glycoprotein VI and platelet adhesion on atherosclerosis--a possible role of fibronectin. Journal of Molecular and Cellular Cardiology, 2010. 49(3): p. 532-42.

69. Kirchhofer, D., M.A. Riederer, and H.R. Baumgartner, Specific Accumulation of Circulating Monocytes and Polymorphonuclear Leukocytes on Platelet Thrombi in a Vascular Injury Model. Blood, 1997. 89(4): p. 1270-1278.

70. Massberg, S., et al., Platelet adhesion via glycoprotein IIb integrin is critical for atheroprogression and focal cerebral ischemia: an in vivo study in mice lacking glycoprotein I/b. Circulation, 2005. 112(8): p. 1180-8.

71. Dong, Z.M., A.A. Brown, and D.D. Wagner, Prominent role of P-selectin in the development of advanced atherosclerosis in ApoE-deficient mice. Circulation, 2000. 101(19): p. 2290-5. 
References.

72. Tardif, J.C., et al., Effects of the P-selectin antagonist inclacumab on myocardial damage after percutaneous coronary intervention for non-ST-segment elevation myocardial infarction: results of the SELECT-ACS trial. J Am Coll Cardiol, 2013. 61(20): p. 2048-55.

73. Stahli, B.E., et al., Effects of the P-Selectin Antagonist Inclacumab on Myocardial Damage After Percutaneous Coronary Intervention According to Timing of Infusion: Insights From the SELECT-ACS Trial. Journal of the American Heart Association, 2016. 5(11).

74. Furman, M.I., et al., Circulating monocyte-platelet aggregates are an early marker of acute myocardial infarction. Journal of the American College of Cardiology, 2001. 38(4): p. 1002-1006.

75. Sarma, J., et al., Increased platelet binding to circulating monocytes in acute coronary syndromes. Circulation, 2002. 105(18): p. 2166-71.

76. Liu, Y., et al., Novel Role of Platelets in Mediating Inflammatory Responses and Ventricular Rupture or Remodeling Following Myocardial Infarction. Arteriosclerosis, Thrombosis, and Vascular Biology, 2011. 31(4): p. 834-841.

77. Furman, M.I., et al., Circulating monocyte-platelet aggregates are an early marker of acute myocardial infarction. Journal of the American College of Cardiology, 2001. 38(4): p. 1002-1006.

78. Dole, V.S., et al., Activated platelets induce Weibel-Palade-body secretion and leukocyte rolling in vivo: role of P-selectin. Blood, 2005. 106(7): p. 2334-9.

79. van Gils, J.M., J.J. Zwaginga, and P.L. Hordijk, Molecular and functional interactions among monocytes, platelets, and endothelial cells and their relevance for cardiovascular diseases. Journal of Leukocyte Biology, 2009. 85(2): p. 195-204.

80. Lievens, D., et al., The multi-functionality of CD4OL and its receptor CD40 in atherosclerosis. Thromb Haemost, 2009. 102(2): p. 206-14.

81. Henn, V., et al., CD40 ligand on activated platelets triggers an inflammatory reaction of endothelial cells. Nature, 1998. 391(6667): p. 591-594.

82. Gawaz, M., et al., Activated platelets induce monocyte chemotactic protein-1 secretion and surface expression of intercellular adhesion molecule-1 on endothelial cells. Circulation, 1998. 98(12): p. 1164-71.

83. Napoleao, P., et al., Stratification of ST-elevation myocardial infarction patients based on soluble CD4OL longitudinal changes. Transl Res, 2016. 176: p. 95-104.

84. Celi, A., et al., P-selectin induces the expression of tissue factor on monocytes. Proceedings of the National Academy of Sciences, 1994. 91(19): p. 8767-71.

85. Palabrica, T., et al., Leukocyte accumulation promoting fibrin deposition is mediated in vivo by P-selectin on adherent platelets. Nature, 1992. 359(6398): p. 848-51.

86. Neumann, F.J., et al., Induction of cytokine expression in leukocytes by binding of thrombin-stimulated platelets. Circulation, 1997. 95(10): p. 2387-94.

87. Weyrich, A.S., et al., Activated platelets signal chemokine synthesis by human monocytes. Journal of Clinical Investigations, 1996. 97(6): p. 1525-34.

88. Schall, T.J., et al., Selective attraction of monocytes and Tlymphocytes of the memory phenotype by cytokine RANTES. Nature, 1990. 347(6294): p. 669-71.

89. Koenen, R.R., et al., Disrupting functional interactions between platelet chemokines inhibits atherosclerosis in hyperlipidemic mice. Nature Medicine, 2009. 15(1): p. 97-103. 
References.

90. Braunersreuther, V., et al., Chemokine CCL5/RANTES inhibition reduces myocardial reperfusion injury in atherosclerotic mice. J Mol Cell Cardiol, 2010. 48(4): p. 789-98.

91. Merhi-Soussi, F., et al., Interleukin-1 plays a major role in vascular inflammation and atherosclerosis in male apolipoprotein E-knockout mice. Cardiovasc Res, 2005. 66(3): p. 583-93.

92. Lievens, D. and P. von Hundelshausen, Platelets in atherosclerosis. Thromb Haemost, 2011. 106(5): p. 827-38.

93. Karras, C., et al., Acute coronary syndromes. Disease-a-month, 2013. 59(5): p. $202-9$.

94. O'Gara, P.T., et al., 2013 ACCF/AHA guideline for the management of ST-elevation myocardial infarction: executive summary: a report of the American College of Cardiology Foundation/American Heart Association Task Force on Practice Guidelines. Circulation, 2013. 127(4): p. 529-55.

95. Keeley, E.C., J.A. Boura, and C.L. Grines, Primary angioplasty versus intravenous thrombolytic therapy for acute myocardial infarction: a quantitative review of 23 randomised trials. Lancet, 2003. 361(9351): p. 13-20.

96. Braunwald, E., et al., ACC/AHA Guidelines for the Management of Patients With Unstable Angina and Non-ST-Segment Elevation Myocardial Infarction: Executive Summary and Recommendations. A Report of the American College of Cardiology/American Heart Association Task Force on Practice Guidelines (Committee on the Management of Patients With Unstable Angina), 2000. 102(10): p. 1193-1209.

97. Patrono, C., et al., Antiplatelet agents for the treatment and prevention of atherothrombosis. European Heart Journal, 2011. 32(23): p. 2922-2932.

98. Juul-Moller, S., et al., Double-blind trial of aspirin in primary prevention of myocardial infarction in patients with stable chronic angina pectoris. The Swedish Angina Pectoris Aspirin Trial (SAPAT) Group. Lancet, 1992. 340(8833): p. 1421-5.

99. Lewis, H.D., Jr., et al., Protective effects of aspirin against acute myocardial infarction and death in men with unstable angina. Results of a Veterans Administration Cooperative Study. New England Journal of Medicine, 1983. 309(7): p. 396-403.

100. Risk of myocardial infarction and death during treatment with low dose aspirin and intravenous heparin in men with unstable coronary artery disease. The RISC Group. Lancet, 1990. 336(8719): p. 827-30.

101. Roux, S., S. Christeller, and E. Lüdin, Effects of aspirin on coronary reocclusion and recurrent ischemia after thrombolysis: A meta-analysis. Journal of the American College of Cardiology, 1992. 19(3): p. 671-677.

102. New Zealand 2012 guidelines for the management of non ST-elevation acute coronary syndromes. The New Zealand Medical Journal, 2012. 125(1357): p. 122-47.

103. ST-elevation myocardial infarction: New Zealand management guidelines, 2013. New Zealand Medical Journal, 2013. 126(1387).

104. Amsterdam, E.A., et al., 2014 AHA/ACC guideline for the management of patients with non-ST-elevation acute coronary syndromes: a report of the American College of Cardiology/American Heart Association Task Force on Practice Guidelines. Circulation, 2014. 130(25): p. e344-426.

105. Roffi, M., et al., 2015 ESC Guidelines for the management of acute coronary syndromes in patients presenting without persistent ST-segment elevation: Task Force for the 
References.

Management of Acute Coronary Syndromes in Patients Presenting without Persistent STSegment Elevation of the European Society of Cardiology (ESC). European Heart Journal, 2015.

106. Steg, P.G., et al., ESC Guidelines for the management of acute myocardial infarction in patients presenting with ST-segment elevation. European Heart Journal, 2012. 33(20): p. 2569-619.

107. Mehta, S.R. and S. Yusuf, The Clopidogrel in Unstable angina to prevent Recurrent Events (CURE) trial programme; rationale, design and baseline characteristics including a metaanalysis of the effects of thienopyridines in vascular disease. European Heart Journal, 2000. 21(24): p. 2033-41.

108. Steinhubl, S.R., et al., Early and sustained dual oral antiplatelet therapy following percutaneous coronary intervention: a randomized controlled trial. Jama, 2002. 288(19): p. 2411-20.

109. Sabatine, M.S., et al., Addition of Clopidogrel to Aspirin and Fibrinolytic Therapy for Myocardial Infarction with ST-Segment Elevation. New England Journal of Medicine, 2005. 352(12): p. 1179-1189.

110. Toma, N., Randomized double-blind assessment of the ONSET and OFFSET of the antiplatelet effects of Ticagrelor versus Clopidogrel in patients with stable coronary artery disease. The ONSET/OFFSET study. Maedica (Buchar), 2010. 5(1): p. 75-6.

111. Wallentin, L., et al., Ticagrelor versus Clopidogrel in Patients with Acute Coronary Syndromes. New England Journal of Medicine, 2009. 361(11): p. 1045-1057.

112. Wiviott, S.D., et al., Prasugrel versus Clopidogrel in Patients with Acute Coronary Syndromes. New England Journal of Medicine, 2007. 357(20): p. 2001-2015.

113. Valenti, R., et al., Residual platelet reactivity to predict long-term clinical outcomes after clopidogrel loading in patients with acute coronary syndromes: comparison of different cutoff values by light transmission aggregometry from the responsiveness to clopidogrel and stent thrombosis 2-acute coronary syndrome (RECLOSE 2-ACS) study. Journal of Thrombosis and Thrombolysis, 2015. 40(1): p. 76-82.

114. Tantry, U.S., et al., Consensus and update on the definition of on-treatment platelet reactivity to adenosine diphosphate associated with ischemia and bleeding. Journal of the American College of Cardiology

2013. 62(24): p. 2261-73.

115. Jaremo, P., et al., Individual variations of platelet inhibition after loading doses of clopidogrel. Journal of Internal Medicine, 2002. 252(3): p. 233-8.

116. Gurbel, P.A., et al., Clopidogrel for coronary stenting: response variability, drug resistance, and the effect of pretreatment platelet reactivity. Circulation, 2003. 107(23): p. 2908-13.

117. Matetzky, S., et al., Clopidogrel resistance is associated with increased risk of recurrent atherothrombotic events in patients with acute myocardial infarction. Circulation, 2004. 109(25): p. 3171-5.

118. Price, M.J., et al., Prognostic significance of post-clopidogrel platelet reactivity assessed by a point-of-care assay on thrombotic events after drug-eluting stent implantation. European Heart Journal, 2008. 29(8): p. 992-1000. 
References.

119. Harding, S., et al., Contemporary antiplatelet therapy in acute coronary syndromes: are there differences in outcomes and discontinuation between clopidogrel and ticagrelor? Internal Medical Journal, 2017. 47(11): p. 1298-1305.

120. Verdoia, M., et al., Prevalence and predictors of high-on treatment platelet reactivity with ticagrelor in ACS patients undergoing stent implantation. Vascular Pharmacology, 2015(0).

121. Lemesle, G., et al., High on-treatment platelet reactivity with ticagrelor versus prasugrel: a systematic review and meta-analysis. Journal of Thrombosis and Haemostasis, 2015. 13(6): p. 931-42.

122. Tentzeris, I., et al., Platelet function variability and non-genetic causes. Thromb Haemost, 2011. 105 Suppl 1: p. S60-6.

123. Badr Eslam, R., et al., Residual platelet activation through protease-activated receptors (PAR)-1 and -4 in patients on P2Y12 inhibitors. International Journal of Cardiology, 2013. 168(1): p. 403-6.

124. Wadowski, P.P., et al., Disaggregation Following Agonist-Induced Platelet Activation in Patients on Dual Antiplatelet Therapy. Journal of Cardiovascular Translational Research, 2017. 10(4): p. 359-367.

125. Kerrigan, S.W., et al., A role for glycoprotein Ib in Streptococcus sanguis-induced platelet aggregation. Blood, 2002. 100(2): p. 509-516.

126. O'Brien, L., et al., Multiple mechanisms for the activation of human platelet aggregation by Staphylococcus aureus: roles for the clumping factors CIfA and CIfB, the serineaspartate repeat protein SdrE and protein A. Mol Microbiol, 2002. 44(4): p. 1033-44.

127. Bhakdi, S., et al., Staphylococcal $\alpha$ toxin promotes blood coagulation via attack on human platelets. The Journal of Experimental Medicine, 1988. 168(2): p. 527-542.

128. Shannon, O., M. Morgelin, and M. Rasmussen, Platelet activation and biofilm formation by Aerococcus urinae, an endocarditis-causing pathogen. Infect Immun, 2010. 78(10): p. 4268-75.

129. Kreutz, R.P., et al., Viral respiratory tract infections increase platelet reactivity and activation: an explanation for the higher rates of myocardial infarction and stroke during viral illness. Journal of Thrombosis and Haemostasis, 2005. 3(9): p. 2108-9.

130. Kreutz, R.P., et al., Inflammatory changes during the 'common cold' are associated with platelet activation and increased reactivity of platelets to agonists. Blood Coagul Fibrinolysis, 2007. 18(8): p. 713-8.

131. Elizalde, J.I., et al., Platelet activation In mice and human Helicobacter pylori infection. Journal of Clinical Investigation, 1997. 100(5): p. 996-1005.

132. Ahn, E.R., et al., Platelet activation in Helicobacter pylori-associated idiopathic thrombocytopenic purpura: eradication reduces platelet activation but seldom improves platelet counts. Acta Haematol, 2006. 116(1): p. 19-24.

133. Yeh, J.J., et al., P-selectin-dependent platelet aggregation and apoptosis may explain the decrease in platelet count during Helicobacter pylori infection. Blood, 2010. 115(21): p. 4247-53.

134. Mulvihill, N.T. and J.B. Foley, Inflammation in acute coronary syndromes. Heart, 2002. 87(3): p. 201-204. 
References.

135. Zakynthinos, E. and N. Pappa, Inflammatory biomarkers in coronary artery disease. Journal of Cardiology, 2009. 53(3): p. 317-333.

136. Lemaitre, B., et al., The dorsoventral regulatory gene cassette spatzle/Toll/cactus controls the potent antifungal response in Drosophila adults. Cell, 1996. 86(6): p. 973-83.

137. Medzhitov, R., P. Preston-Hurlburt, and C.A. Janeway, A human homologue of the Drosophila Toll protein signals activation of adaptive immunity. Nature, 1997. 388(6640): p. 394-397.

138. Hirsiger, S., et al., Danger signals activating the immune response after trauma. Mediators Inflamm, 2012. 2012: p. 315941.

139. Schiopu, A. and O.S. Cotoi, S100A8 and S100A9: DAMPs at the crossroads between innate immunity, traditional risk factors, and cardiovascular disease. Mediators Inflamm, 2013. 2013: p. 828354.

140. Alvarez, K. and G. Vasquez, Damage-associated molecular patterns and their role as initiators of inflammatory and auto-immune signals in systemic lupus erythematosus. International Reviews of Immunology, 2017. 36(5): p. 259-270.

141. Goh, F.G. and K.S. Midwood, Intrinsic danger: activation of Toll-like receptors in rheumatoid arthritis. Rheumatology, 2012. 51(1): p. 7-23.

142. Takeda, K., O. Takeuchi, and S. Akira, Recognition of lipopeptides by Toll-like receptors. Journal of Endotoxin Research

2002. 8(6): p. 459-63.

143. Janot, L., et al., CD14 Works with Toll-Like Receptor 2 to Contribute to Recognition and Control of Listeria monocytogenes Infection. Journal of Infectious Diseases, 2008. 198(1): p. 115-124.

144. Triantafilou, M., et al., Membrane sorting of toll-like receptor (TLR)-2/6 and TLR2/1 heterodimers at the cell surface determines heterotypic associations with CD36 and intracellular targeting. J Biol Chem, 2006. 281(41): p. 31002-11.

145. Gantner, B.N., et al., Collaborative Induction of Inflammatory Responses by Dectin-1 and Toll-like Receptor 2. The Journal of Experimental Medicine, 2003. 197(9): p. 1107-1117.

146. Yu, M., et al., HMGB1 signals through toll-like receptor (TLR) 4 and TLR2. Shock, 2006. 26(2): p. 174-9.

147. Asea, A., et al., Novel signal transduction pathway utilized by extracellular HSP70: role of toll-like receptor (TLR) 2 and TLR4. J Biol Chem, 2002. 277(17): p. 15028-34.

148. Rietschel, E.T. and H. Brade, Bacterial endotoxins. Scientific American, 1992. 267(2): p. 54-61.

149. Fenton, M.J. and D.T. Golenbock, LPS-binding proteins and receptors. Journal of Leukocyte Biology, 1998. 64(1): p. 25-32.

150. Shimazu, R., et al., MD-2, a Molecule that Confers Lipopolysaccharide Responsiveness on Toll-like Receptor 4. The Journal of Experimental Medicine, 1999. 189(11): p. 1777-1782.

151. Wittebole, X., et al., Expression of tumour necrosis factor receptor and Toll-like receptor 2 and 4 on peripheral blood leucocytes of human volunteers after endotoxin challenge: a comparison of flow cytometric light scatter and immunofluorescence gating. Clin Exp Immunol, 2005. 141(1): p. 99-106. 
References.

152. Brandl, K., et al., TLR-4 surface display on human monocytes is increased in septic patients. European Journal of Medical Research, 2005. 10(8): p. 319-324.

153. Termeer, C., et al., Oligosaccharides of Hyaluronan activate dendritic cells via toll-like receptor 4. Journal of Experimental Medicine, 2002. 195(1): p. 99-111.

154. Smiley, S.T., J.A. King, and W.W. Hancock, Fibrinogen stimulates macrophage chemokine secretion through toll-like receptor 4. Journal of Immunology, 2001. 167(5): p. 2887-94.

155. Krieg, A.M., Lymphocyte activation by CpG dinucleotide motifs in prokaryotic DNA. Trends Microbiol, 1996. 4(2): p. 73-6.

156. Hemmi, H., et al., A Toll-like receptor recognizes bacterial DNA. Nature, 2000. 408(6813): p. 740-5.

157. Leifer, C.A., et al., TLR9 is Localized in the Endoplasmic Reticulum Prior to Stimulation. Journal of immunology (Baltimore, Md. : 1950), 2004. 173(2): p. 1179-1183.

158. Bamboat, Z.M., et al., Toll-like receptor 9 inhibition confers protection from liver ischemiareperfusion injury. Hepatology, 2010. 51(2): p. 621-32.

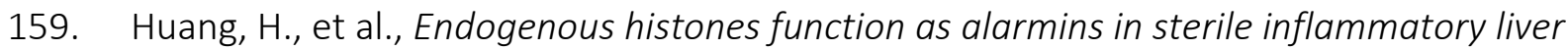
injury through Toll-like receptor 9 in mice. Hepatology, 2011. 54(3): p. 999-1008.

160. Xu, J., et al., Extracellular histones are major mediators of death in sepsis. Nature Medicine, 2009. 15(11): p. 1318-21.

161. Bjorkbacka, H., et al., Reduced atherosclerosis in MyD88-null mice links elevated serum cholesterol levels to activation of innate immunity signaling pathways. Nature Medicine, 2004. 10(4): p. 416-21.

162. Michelsen, K.S., et al., Lack of Toll-like receptor 4 or myeloid differentiation factor 88 reduces atherosclerosis and alters plaque phenotype in mice deficient in apolipoprotein $E$. Proc Natl Acad Sci U S A, 2004. 101(29): p. 10679-84.

163. Liu, X., et al., Toll-like receptor 2 plays a critical role in the progression of atherosclerosis that is independent of dietary lipids. Atherosclerosis, 2008. 196(1): p. 146-54.

164. Madan, M. and S. Amar, Toll-like receptor-2 mediates diet and/or pathogen associated atherosclerosis: proteomic findings. PLoS One, 2008. 3(9): p. e3204.

165. Mullick, A.E., P.S. Tobias, and L.K. Curtiss, Modulation of atherosclerosis in mice by Tolllike receptor 2. J Clin Invest, 2005. 115(11): p. 3149-56.

166. Mullick, A.E., et al., Increased endothelial expression of Toll-like receptor 2 at sites of disturbed blood flow exacerbates early atherogenic events. Journal of Experimental Medicine, 2008. 205(2): p. 373-83.

167. Warboys, C.M., et al., The role of blood flow in determining the sites of atherosclerotic plaques. F1000 Medicine Reports, 2011. 3: p. 5.

168. $\mathrm{Xu}, \mathrm{X} . \mathrm{H}$., et al., Toll-like receptor-4 is expressed by macrophages in murine and human lipid-rich atherosclerotic plaques and upregulated by oxidized LDL. Circulation, 2001. 104(25): p. 3103-8.

169. Vink, A., et al., In vivo evidence for a role of toll-like receptor 4 in the development of intimal lesions. Circulation, 2002. 106(15): p. 1985-90.

170. Curtiss, L.K., et al., Atherosclerosis induced by endogenous and exogenous toll-like receptor (TLR)1 or TLR6 agonists. Journal of Lipid Research, 2012. 53(10): p. 2126-32.

171. Edfeldt, K., et al., Expression of toll-like receptors in human atherosclerotic lesions: a possible pathway for plaque activation. Circulation, 2002. 105(10): p. 1158-61. 
References.

172. Mullick, A.E., P.S. Tobias, and L.K. Curtiss, Modulation of atherosclerosis in mice by Tolllike receptor 2. Journal of Clinical Investigation, 2005. 115(11): p. 3149-56.

173. Dunzendorfer, S., H.K. Lee, and P.S. Tobias, Flow-dependent regulation of endothelial Tolllike receptor 2 expression through inhibition of SP1 activity. Circulation Research, 2004. 95(7): p. 684-91.

174. Monaco, C., et al., Toll-like receptor-2 mediates inflammation and matrix degradation in human atherosclerosis. Circulation, 2009. 120(24): p. 2462-9.

175. Kiechl, S., et al., Toll-like Receptor 4 Polymorphisms and Atherogenesis. New England Journal of Medicine, 2002. 347(3): p. 185-192.

176. Levonen, A.-L., et al., Antioxidant Gene Therapy for Cardiovascular Disease. Current Status and Future Perspectives, 2008. 117(16): p. 2142-2150.

177. Sharma, H.S. and D.K. Das, Role of cytokines in myocardial ischemia and reperfusion. Mediators of Inflammation, 1997. 6(3): p. 175-183.

178. Shishido, T., et al., Toll-like receptor-2 modulates ventricular remodeling after myocardial infarction. Circulation, 2003. 108(23): p. 2905-10.

179. Sakata, Y., et al., Toll-like receptor 2 modulates left ventricular function following ischemia-reperfusion injury. Am J Physiol Heart Circ Physiol, 2007. 292(1): p. H503-9.

180. Favre, J., et al., Toll-like receptors 2-deficient mice are protected against postischemic coronary endothelial dysfunction. Arterioscler Thromb Vasc Biol, 2007. 27(5): p. 1064-71.

181. Oyama, J., et al., Reduced myocardial ischemia-reperfusion injury in toll-like receptor 4deficient mice. Circulation, 2004. 109(6): p. 784-9.

182. Chong, A.J., et al., Toll-like receptor 4 mediates ischemia/reperfusion injury of the heart. J Thorac Cardiovasc Surg, 2004. 128(2): p. 170-9.

183. Shimamoto, A., et al., Inhibition of Toll-like receptor 4 with eritoran attenuates myocardial ischemia-reperfusion injury. Circulation, 2006. 114(1 Suppl): p. 1270-4.

184. Blair, P., et al., Stimulation of Toll-like receptor 2 in human platelets induces a thromboinflammatory response through activation of phosphoinositide 3-kinase. Circulation Research, 2009. 104(3): p. 346-54.

185. Shiraki, R., et al., Expression of Toll-like receptors on human platelets. Thrombosis Research, 2004. 113(6): p. 379-85.

186. Koupenova, M., et al., Sex differences in platelet toll-like receptors and their association with cardiovascular risk factors. Arterioscler Thromb Vasc Biol, 2015. 35(4): p. 1030-7.

187. Cognasse, F., et al., Evidence of Toll-like receptor molecules on human platelets. Immunology and Cell Biology, 2005. 83(2): p. 196-8.

188. Aslam, R., et al., Platelet Toll-like receptor expression modulates lipopolysaccharideinduced thrombocytopenia and tumor necrosis factor-alpha production in vivo. Blood, 2006. 107(2): p. 637-41.

189. Rivadeneyra, L., et al., Regulation of platelet responses triggered by Toll-like receptor 2 and 4 ligands is another non-genomic role of nuclear factor-kappaB. Thromb Res, 2014. 133(2): p. 235-43.

190. Rex, S., et al., Immune versus thrombotic stimulation of platelets differentially regulates signalling pathways, intracellular protein-protein interactions, and alpha-granule release. Thromb Haemost, 2009. 102(1): p. 97-110. 
References.

191. Klarstrom Engstrom, K., et al., The role of Porphyromonas gingivalis gingipains in platelet activation and innate immune modulation. Mol Oral Microbiol, 2015. 30(1): p. 62-73.

192. Assinger, A., et al., Efficient phagocytosis of periodontopathogens by neutrophils requires plasma factors, platelets and TLR2. Journal of Thrombosis and Haemostasis, 2011. 9(4): p. 799-809.

193. Falker, K., et al., The toll-like receptor 2/1 (TLR2/1) complex initiates human platelet activation via the src/Syk/LAT/PLCgamma2 signalling cascade. Cell Signal, 2014. 26(2): p. 279-86.

194. Klarstrom Engstrom, K., et al., Toll like receptor 2/1 mediated platelet adhesion and activation on bacterial mimetic surfaces is dependent on src/Syk-signaling and purinergic receptor P2X1 and P2Y12 activation. Biointerphases, 2014. 9(4): p. 041003.

195. Yanaga, F., et al., Syk interacts with tyrosine-phosphorylated proteins in human platelets activated by collagen and cross-linking of the Fc gamma-IIA receptor. Biochemical Journal, 1995. 311(Pt 2): p. 471-478.

196. Assinger, A., et al., Periodontopathogens induce expression of CD4OL on human platelets via TLR2 and TLR4. Thrombosis Research, 2012. 130(3): p. e73-e78.

197. Ståhl, A.-I., et al., Lipopolysaccharide from enterohemorrhagic Escherichia coli binds to platelets through TLR4 and CD62 and is detected on circulating platelets in patients with hemolytic uremic syndrome. Blood, 2006. 108(1): p. 167-176.

198. Zhang, G., et al., Lipopolysaccharide stimulates platelet secretion and potentiates platelet aggregation via TLR4/MyD88 and the CGMP-dependent protein kinase pathway. Journal of Immunology, 2009. 182(12): p. 7997-8004.

199. Andonegui, G., et al., Platelets express functional Toll-like receptor-4. Blood, 2005. 106(7): p. 2417-23.

200. Ward, J.R., et al., Agonists of toll-like receptor (TLR)2 and TLR4 are unable to modulate platelet activation by adenosine diphosphate and platelet activating factor. Thromb Haemost, 2005. 94(4): p. 831-8.

201. Shashkin, P.N., et al., Lipopolysaccharide is a direct agonist for platelet RNA splicing. Journal of Immunology, 2008. 181(5): p. 3495-502.

202. Sheu, J.R., et al., Mechanisms involved in the antiplatelet activity of Escherichia coli lipopolysaccharide in human platelets. British Journal of Haemotology, 1998. 103(1): p. 29-38.

203. Semeraro, F., et al., Extracellular histones promote thrombin generation through plateletdependent mechanisms: involvement of platelet TLR2 and TLR4. Blood, 2011. 118(7): p. 1952-61.

204. Cognasse, F., et al., Lipopolysaccharide induces SCD4OL release through human platelets TLR4, but not TLR2 and TLR9. Intensive Care Med, 2007. 33(2): p. 382-4.

205. Inwald, D.P., et al., Platelet and soluble CD4OL in meningococcal sepsis. Intensive Care Med, 2006. 32(9): p. 1432-7.

206. Brown, G.T. and T.M. McIntyre, Lipopolysaccharide signaling without a nucleus: kinase cascades stimulate platelet shedding of proinflammatory IL-1beta-rich microparticles. Journal of Immunology, 2011. 186(9): p. 5489-96. 
References.

207. Xiong, J., et al., Leukocyte- and platelet-derived microvesicle interactions following in vitro and in vivo activation of toll-like receptor 4 by lipopolysaccharide. PLoS One, 2011. 6(9): p. e25504.

208. Calvano, J.E., et al., Modulation of the lipopolysaccharide receptor complex (CD14, TLR4, $M D$-2) and toll-like receptor 2 in systemic inflammatory response syndrome-positive patients with and without infection: relationship to tolerance. Shock, 2003. 20(5): p. 4159.

209. Damien, P., et al., LPS stimulation of purified human platelets is partly dependent on plasma soluble CD14 to secrete their main secreted product, soluble-CD40-Ligand. BMC Immunology, 2015. 16(1): p. 3.

210. Damien, P., et al., Highly active antiretroviral therapy alters inflammation linked to platelet cytokines in HIV-1-infected patients. J Infect Dis, 2013. 208(5): p. 868-70.

211. Cognasse, F., et al., Toll-like receptor 4 ligand can differentially modulate the release of cytokines by human platelets. Br J Haematol, 2008. 141(1): p. 84-91.

212. Pugin, J., et al., Lipopolysaccharide activation of human endothelial and epithelial cells is mediated by lipopolysaccharide-binding protein and soluble CD14. Proc Natl Acad Sci U S A, 1993. 90(7): p. 2744-8.

213. Spinelli, S.L., et al., Platelets and megakaryocytes contain functional nuclear factorkappaB. Arteriosclerosis, Thrombosis and Vascular Biology, 2010. 30(3): p. 591-8.

214. Berthet, J., et al., Toll-like receptor 4 signal transduction in platelets: novel pathways. British Journal of Haematology, 2010. 151(1): p. 89-92.

215. Thon, J.N., et al., T granules in human platelets function in TLR9 organization and signaling. Journal of Cell Biology, 2012. 198(4): p. 561-74.

216. Panigrahi, S., et al., Engagement of platelet toll-like receptor 9 by novel endogenous ligands promotes platelet hyperreactivity and thrombosis. Circulation Research, 2013. 112(1): p. 103-12.

217. Sims, J.B., et al., Urinary tract infection in patients with acute coronary syndrome: A potential systemic inflammatory connection. American Heart Journal, 2005. 149(6): p. 1062-1065.

218. Naghavi, M., et al., Influenza infection exerts prominent inflammatory and thrombotic effects on the atherosclerotic plaques of apolipoprotein E-deficient mice. Circulation, 2003. 107(5): p. 762-8.

219. Madjid, M., et al., Systemic Infections Cause Exaggerated Local Inflammation in Atherosclerotic Coronary Arteries: Clues to the Triggering Effect of Acute Infections on Acute Coronary Syndromes. Texas Heart Institute Journal, 2007. 34(1): p. 11-18.

220. Ezzahiri, R., et al., Chlamydia pneumoniae infection induces an unstable atherosclerotic plaque phenotype in LDL-receptor, ApoE double knockout mice. European Journal of Vascular and Endovascular Surgery, 2003. 26(1): p. 88-95.

221. Vliegen, I., et al., Cytomegalovirus infection aggravates atherogenesis in apoE knockout mice by both local and systemic immune activation. Microbes and Infection, 2004. 6(1): p. 17-24.

222. Hayashi, C., et al., Porphyromonas gingivalis accelerates inflammatory atherosclerosis in the innominate artery of ApoE deficient mice. Atherosclerosis, 2011. 215(1): p. 52-9. 
References.

223. Visser, M.R., et al., Enhanced thrombin generation and platelet binding on herpes simplex virus-infected endothelium. Proceedings of the National Academy of Sciences of the United States of America, 1988. 85(21): p. 8227-30.

224. Key, N.S., et al., Infection of vascular endothelial cells with herpes simplex virus enhances tissue factor activity and reduces thrombomodulin expression. Proceedings of the National Academy of Sciences of the United States of America, 1990. 87(18): p. 7095-9.

225. van Dam-Mieras, M.C., et al., The procoagulant response of cytomegalovirus infected endothelial cells. Thromb Haemost, 1992. 68(3): p. 364-70.

226. Mattila, K.J., Viral and bacterial infections in patients with acute myocardial infarction. Journal of Internal Medicine, 1989. 225(5): p. 293-6.

227. Clayton, T.C., M. Thompson, and T.W. Meade, Recent respiratory infection and risk of cardiovascular disease: case-control study through a general practice database. European Heart Journal, 2008. 29(1): p. 96-103.

228. Spodick, D.H., A.P. Flessas, and M.M. Johnson, Association of acute respiratory symptoms with onset of acute myocardial infarction: Prospective investigation of 150 consecutive patients and matched control patients. The American Journal of Cardiology, 1984. 53(4): p. 481-482.

229. Penttinen, J. and P. Valonen, The risk of myocardial infarction among Finnish farmers seeking medical care for an infection. American Journal of Public Health, 1996. 86(10): p. 1440-1442.

230. Meier, C.R., et al., Acute respiratory-tract infections and risk of first-time acute myocardial infarction. Lancet, 1998. 351(9114): p. 1467-71.

231. Meyers, D.G., et al., Influenza and Pneumococcal Vaccinations Fail to Prevent Myocardial Infarction. Heart Drug, 2004. 4(2): p. 96-100.

232. Madjid, M. and S.W. Casscells, Of birds and men: cardiologists' role in influenza pandemics. The Lancet, 2004. 364(9442): p. 1309.

233. Collins, S.D., Excess Mortality from Causes Other than Influenza and Pneumonia during Influenza Epidemics. Public Health Reports (1896-1970), 1932. 47(46): p. 2159-2179.

234. Madjid, M., et al., Influenza epidemics and acute respiratory disease activity are associated with a surge in autopsy-confirmed coronary heart disease death: results from 8 years of autopsies in 34,892 subjects. European Heart Journal, 2007. 28(10): p. 1205-10.

235. Musher, D.M., et al., The association between pneumococcal pneumonia and acute cardiac events. Clin Infect Dis, 2007. 45(2): p. 158-65.

236. Corrales-Medina, V.F., et al., Acute bacterial pneumonia is associated with the occurrence of acute coronary syndromes. Medicine (Baltimore), 2009. 88(3): p. 154-9.

237. Ramirez, J., et al., Acute myocardial infarction in hospitalized patients with communityacquired pneumonia. Clin Infect Dis, 2008. 47(2): p. 182-7.

238. Swampillai, J.W., N; Sebastian, C; Devlin, G P, Seasonal Variations in Hospital Admissions for ST-Elevation Myocardial Infarction in New Zealand. Cardiology Research, 2012. 5(5): p. 205-208.

239. Hopstock, L.A., et al., Seasonal variation in incidence of acute myocardial infarction in a sub-Arctic population: the Tromso Study 1974-2004. Eur J Cardiovasc Prev Rehabil, 2011. 18(2): p. 320-5. 
References.

240. Sheth, T., et al., Increased winter mortality from acute myocardial infarction and stroke: The effect of age. Journal of the American College of Cardiology, 1999. 33(7): p. 1916 1919.

241. Sharovsky, R. and L.A.M. César, Increase in mortality due to myocardial infarction in the Brazilian city of São Paulo during winter. Arquivos Brasileiros de Cardiologia, 2002. 78: p. 106-109.

242. Angiolillo, D.J., et al., Identification of low responders to a 300-mg clopidogrel loading dose in patients undergoing coronary stenting. Thrombosis Research, 2005. 115(1-2): p. 101-8.

243. Sharma, R.K., et al., Aspirin and clopidogrel hyporesponsiveness and nonresponsiveness in patients with coronary artery stenting. Journal of Vascular Health and Risk Management, 2009. 5: p. 965-72.

244. Clark, S.R., et al., Platelet TLR4 activates neutrophil extracellular traps to ensnare bacteria in septic blood. Nat Med, 2007. 13(4): p. 463-9.

245. Koupenova, M., et al., Platelet-TLR7 mediates host survival and platelet count during viral infection in the absence of platelet-dependent thrombosis. Blood, 2014. 124(5): p. 791802.

246. Takeshita, F., et al., Cutting edge: Role of Toll-like receptor 9 in CpG DNA-induced activation of human cells. Journal of Immunology, 2001. 167(7): p. 3555-8.

247. Thygesen, K., et al., Third Universal Definition of Myocardial Infarction. Circulation, 2012. 126(16): p. 2020-2035.

248. Aradi, D., et al., Prognostic significance of high on-clopidogrel platelet reactivity after percutaneous coronary intervention: systematic review and meta-analysis. American Heart Journal, 2010. 160(3): p. 543-51.

249. Massberg, S., C. Schulz, and M. Gawaz, Role of platelets in the pathophysiology of acute coronary syndrome. Semin Vasc Med, 2003. 3(2): p. 147-62.

250. Meng, W., et al., Nuclease-resistant immunostimulatory phosphodiester CpG oligodeoxynucleotides as human Toll-like receptor 9 agonists. BMC Biotechnol, 2011. 11: p. 88.

251. Jiang, W., et al., TLR9 stimulation drives naive B cells to proliferate and to attain enhanced antigen presenting function. European Journal of Immunoloby, 2007. 37(8): p. 2205-13.

252. Hornung, V., et al., Quantitative expression of toll-like receptor 1-10 mRNA in cellular subsets of human peripheral blood mononuclear cells and sensitivity to CpG oligodeoxynucleotides. Journal of Immunology, 2002. 168(9): p. 4531-7.

253. Zarember, K.A. and P.J. Godowski, Tissue expression of human Toll-like receptors and differential regulation of Toll-like receptor mRNAs in leukocytes in response to microbes, their products, and cytokines. Journal of Immunology, 2002. 168(2): p. 554-61.

254. Hayashi, F., T.K. Means, and A.D. Luster, Toll-like receptors stimulate human neutrophil function. Blood, 2003. 102(7): p. 2660-9.

255. Verthelyi, D., et al., Human peripheral blood cells differentially recognize and respond to two distinct CPG motifs. Journal of Immunology, 2001. 166(4): p. 2372-7.

256. Krieg, A.M., et al., CPG motifs in bacterial DNA trigger direct B-cell activation. Nature, 1995. 374(6522): p. 546-9. 
References.

257. Iho, S., et al., Oligodeoxynucleotides containing palindrome sequences with internal 5'CpG-3' act directly on human NK and activated T cells to induce IFN-gamma production in vitro. Journal of Immunology, 1999. 163(7): p. 3642-52.

258. Hartmann, G. and A.M. Krieg, CpG DNA and LPS induce distinct patterns of activation in human monocytes. Gene Therapy, 1999. 6(5): p. 893-903.

259. Todoroki, N., et al., Enhancement by IL-16 and IFN- $\gamma$ of platelet activation: Adhesion to leukocytes via GMP-140 / padgem protein (CD62). Biochemical and Biophysical Research Communications, 1991. 179(2): p. 756-761.

260. Danese, S., et al., Cutting edge: T cells trigger CD40-dependent platelet activation and granular RANTES release: a novel pathway for immune response amplification. Journal of Immunology, 2004. 172(4): p. 2011-5.

261. González Hernández, E., et al., Seasonal variations in admissions for acute myocardial infarction. The PRIMVAC Study. Revista Española de Cardiología (English Version), 2004. 57(01): p. 12-19.

262. Hopstock, L.A., et al., Seasonal variation in incidence of acute myocardial infarction in a sub-Arctic population: the Tromso Study 1974-2004. European Journal of Cardiovascular Prevention and Rehabilitation, 2011. 18(2): p. 320-5.

263. Sheth, T., et al., Increased winter mortality from acute myocardial infarction and stroke: the effect of age. Journal of the American College of Cardiology, 1999. 33(7): p. 1916-9.

264. Corrales-Medina, V.F., M. Madjid, and D.M. Musher, Role of acute infection in triggering acute coronary syndromes. The Lancet Infectious Diseases, 2010. 10(2): p. 83-92.

265. Warren-Gash, C., et al., Influenza infection and risk of acute myocardial infarction in England and Wales: A CALIBER self-controlled case series study. Journal of Infectious Diseases, 2012.

266. Hansson, G.K., A.K. Robertson, and C. Soderberg-Naucler, Inflammation and atherosclerosis. Annual Review of Pathology, 2006. 1: p. 297-329.

267. Zhou, J., et al., Heat shock up-regulates expression of Toll-like receptor-2 and Toll-like receptor-4 in human monocytes via p38 kinase signal pathway. Immunology, 2005. 114(4): p. 522-30.

268. Wu, H., et al., TLR4 activation mediates kidney ischemia/reperfusion injury. Journal of Clinical Investigation, 2007. 117(10): p. 2847-59.

269. Yu, L., L. Wang, and S. Chen, Endogenous toll-like receptor ligands and their biological significance. Journal of Cellular and Molecular Medicine, 2010. 14(11): p. 2592-603.

270. Bode, C., et al., CpG DNA as a vaccine adjuvant. Expert review of vaccines, 2011. 10(4): p. 499-511.

271. McCluskie, M.J. and H.L. Davis, CpG DNA is a potent enhancer of systemic and mucosal immune responses against hepatitis $B$ surface antigen with intranasal administration to mice. Journal of Immunology, 1998. 161(9): p. 4463-6.

272. Eastcott, J.W., et al., Oligonucleotide containing CpG motifs enhances immune response to mucosally or systemically administered tetanus toxoid. Vaccine, 2001. 19(13-14): p. 1636-42.

273. Cooper, C.L., et al., CPG 7909, an immunostimulatory TLR9 agonist oligodeoxynucleotide, as adjuvant to Engerix-B HBV vaccine in healthy adults: a double-blind phase I/II study. Journal of Clin Immunology, 2004. 24(6): p. 693-701. 
References.

274. Berthet, J., et al., Toll-like receptor 4 signal transduction in platelets: novel pathways. $\mathrm{Br} \mathrm{J}$ Haematol, 2010. 151(1): p. 89-92.

275. Gurses, K.M., et al., Enhanced Platelet Toll-like Receptor 2 and 4 Expression in Acute Coronary Syndrome and Stable Angina Pectoris. American Journal of Cardiology, 2015. 116(11): p. 1666-1671.

276. Kuwahata, S., et al., High expression level of Toll-like receptor 2 on monocytes is an important risk factor for arteriosclerotic disease. Atherosclerosis, 2010. 209(1): p. 248254.

277. Shao, L., et al., TLR3 and TLR4 as potential clinically biomarkers of cardiovascular risk in coronary artery disease (CAD) patients. Heart Vessels, 2014. 29(5): p. 690-8.

278. Clark, S.R., et al., Platelet TLR4 activates neutrophil extracellular traps to ensnare bacteria in septic blood. Nature Medicine, 2007. 13(4): p. 463-469.

279. Koupenova, M., et al., Platelet-TLR7 mediates host survival and platelet count during viral infection in the absence of platelet-dependent thrombosis. Blood, 2014. 124(5): p. 791802.

280. Rivadeneyra, L., et al., Regulation of platelet responses triggered by Toll-like receptor 2 and 4 ligands is another non-genomic role of nuclear factor-kappaB. Thrombosis Research, 2014. 133(2): p. 235-243.

281. Blair, P., et al., Stimulation of Toll-Like Receptor 2 in Human Platelets Induces a Thromboinflammatory Response Through Activation of Phosphoinositide 3-Kinase. Circulation Research, 2009. 104(3): p. 346-U132.

282. Freedman, J.E. and J. Loscalzo, Platelet-Monocyte Aggregates. Bridging Thrombosis and Inflammation, 2002. 105(18): p. 2130-2132.

283. Cerletti, C., G. de Gaetano, and R. Lorenzet, Platelet - leukocyte interactions: multiple links between inflammation, blood coagulation and vascular risk. Mediterranean Journal of Hematology and Infectious Disseases, 2010. 2(3): p. e2010023.

284. Michelson, A.D., et al., Circulating Monocyte-Platelet Aggregates Are a More Sensitive Marker of In Vivo Platelet Activation Than Platelet Surface P-Selectin. Studies in Baboons, Human Coronary Intervention, and Human Acute Myocardial Infarction, 2001. 104(13): p. 1533-1537.

285. Arumugam, T.V., et al., Toll-like receptors in ischemia-reperfusion injury. Shock, 2009. 32(1): p. 4-16.

286. Mersmann, J., et al., Attenuation of myocardial injury by HMGB1 blockade during ischemia/reperfusion is toll-like receptor 2-dependent. Mediators Inflamm, 2013. 2013: p. 174168.

287. Taylor, K.R., et al., Hyaluronan fragments stimulate endothelial recognition of injury through TLR4. Journal of Biological Chemistry, 2004. 279(17): p. 17079-84.

288. Thomas, M.R. and R.F. Storey, The role of platelets in inflammation. Thromb Haemost, 2015. 114(3): p. 449-58.

289. Koenen, R.R., The prowess of platelets in immunity and inflammation. Thromb Haemost, 2016. 116(4): p. 605-12.

290. Weber, C.H., K.; Zacharowski, K.; Pape, A.; Lindhoff-Last, E.; Moritz, A.; Hanke, A.; Hofstetter, C, Evaluation of multiple electrode aggregometry for the perioperative 
assessment of aspirin therapy in cardiac surgery. Applied Cardiopulmonary Pathophysiology, 2010. 14: p. 163-173.

291. Altman, D.G., Practical Statistics For Medical Research. 1991: Chapman and Hall.

292. FitzGerald, G.A., Mechanisms of platelet activation: thromboxane A2 as an amplifying signal for other agonists. American ofJournal Cardiology, 1991. 68(7): p. 11b-15b.

293. Leon, M.B., et al., A Clinical trial comparing three antithrombotic-drug regimens after coronary-artery stenting. New England Journal of Medicine, 1998. 339(23): p. 1665-1671.

294. Yusuf, S., et al., Effects of clopidogrel in addition to aspirin in patients with acute coronary syndromes without ST-segment elevation. New England Journal of Medicine, 2001. 345(7): p. 494-502.

295. Cattaneo, M., et al., Deficiency of (33P)2MeS-ADP binding sites on platelets with secretion defect, normal granule stores and normal thromboxane A2 production. Evidence that ADP potentiates platelet secretion independently of the formation of large platelet aggregates and thromboxane A2 production. Thromb Haemost, 1997. 77(5): p. 986-90.

296. Storey, R.F., et al., Inhibitory effects of ticagrelor compared with clopidogrel on platelet function in patients with acute coronary syndromes: the PLATO (PLATelet inhibition and patient Outcomes) PLATELET substudy. Journal of the American College of Cardiology, 2010. 56(18): p. 1456-62.

297. Massberg, S., et al., Platelet-Endothelial Cell Interactions During Ischemia/Reperfusion: The Role of P-Selectin. Blood, 1998. 92(2): p. 507-515.

298. Gawaz, M., Role of platelets in coronary thrombosis and reperfusion of ischemic myocardium. Cardiovascular Research, 2004. 61(3): p. 498-511.

299. Massberg, S., et al., Fibrinogen Deposition at the Postischemic Vessel Wall Promotes Platelet Adhesion During Ischemia-Reperfusion In Vivo. Blood, 1999. 94(11): p. 38293838.

300. Timmers, L., et al., Toll-like receptor 4 mediates maladaptive left ventricular remodeling and impairs cardiac function after myocardial infarction. Circ Res, 2008. 102(2): p. 25764.

301. Hally, K.E., et al., Platelet Toll-like receptor (TLR) expression and TLR-mediated platelet activation in acute myocardial infarction. Thrombosis Research, 2017. 158: p. 8-15.

302. Funderburg, N., et al., Toll-Like Receptor Ligands Induce Human T Cell Activation and Death, a Model for HIV Pathogenesis. PLoS ONE, 2008. 3(4): p. e1915.

303. Tincati, C., et al., Role of in vitro stimulation with lipopolysaccharide on T-cell activation in HIV-infected antiretroviral-treated patients. Clin Dev Immunol, 2012. 2012: p. 935425.

304. Maecker, H.T., J.P. McCoy, and R. Nussenblatt, Standardizing immunophenotyping for the Human Immunology Project. Nature reviews. Immunology, 2012. 12(3): p. 191-200.

305. Gros, A., V. Ollivier, and B. Ho-Tin-Noe, Platelets in inflammation: regulation of leukocyte activities and vascular repair. Front Immunol, 2014. 5: p. 678.

306. Tunjungputri, R.N., et al., Differential effects of platelets and platelet inhibition by ticagrelor on TLR2â€" and TLR4-mediated inflammatory responses. Thrombosis and Haemostasis, 2015. 113(5): p. 1035-1045.

307. Hurley, S.M., et al., Platelet-Dependent Neutrophil Function Is Dysregulated by M Protein from Streptococcus pyogenes. Infect Immun, 2015. 83(9): p. 3515-25. 
References.

308. Losche, W., et al., Contact-induced modulation of neutrophil elastase secretion and phagocytic activity by platelets. Blood Coagul Fibrinolysis, 1996. 7(2): p. 210-3.

309. Reinisch, C.M., et al., The inhibition of oxygen radical release from human neutrophils by resting platelets is reversed by administration of acety/salicylic acid or clopidogrel. Free Radic Res, 2001. 34(5): p. 461-6.

310. Le Meur, Y., et al., Whole blood production of monocytic cytokines (IL-16, IL-6, TNF- $\alpha$, s/L$6 R, I L-1 R a)$ in haemodialysed patients. Nephrology Dialysis Transplantation, 1999. 14(10): p. $2420-2426$.

311. Ando, Y., T. Oku, and T. Tsuji, Platelets attenuate production of cytokines and nitric oxide by macrophages in response to bacterial endotoxin. Platelets, 2016. 27(4): p. 344-50.

312. Hagihara, M., et al., Platelets, after exposure to a high shear stress, induce IL-10producing, mature dendritic cells in vitro. Journal of Immunology, 2004. 172(9): p. 5297303.

313. Freeman, B.E., et al., Regulation of innate CD8(+) T-cell activation mediated by cytokines. Proceedings of the National Academy of Sciences of the United States of America, 2012. 109(25): p. 9971-9976.

314. Di Genova, G., et al., Bystander stimulation of activated CD4+ T cells of unrelated specificity following a booster vaccination with tetanus toxoid. European Journal of Immunology, 2010. 40(4): p. 976-85.

315. Chu, T., et al., Bystander-activated memory CD8 T cells control early pathogen load in an innate-like, NKG2D-dependent manner. Cell Reports, 2013. 3(3): p. 701-8.

316. Avice, M.N., et al., IL-15 promotes IL-12 production by human monocytes via T celldependent contact and may contribute to IL-12-mediated IFN-gamma secretion by CD4+ T cells in the absence of TCR ligation. Journal of Immunology, 1998. 161(7): p. 3408-15.

317. Kamath, A.T., C.E. Sheasby, and D.F. Tough, Dendritic cells and NK cells stimulate bystander T cell activation in response to TLR agonists through secretion of IFN-alpha beta and IFN-gamma. Journal of Immunology, 2005. 174(2): p. 767-76.

318. Elzey, B.D., et al., Platelet-derived CD154 enables T-cell priming and protection against $<$ em>Listeria monocytogenes</em> challenge. Blood, 2008. 111(7): p. 3684-3691.

319. Elzey, B.D., et al., Platelet-mediated modulation of adaptive immunity: A communication link between innate and adaptive immune compartments. Immunity, 2003. 19(1): p. 9-19.

320. lannacone, M., et al., Platelets prevent IFN-alpha/beta-induced lethal hemorrhage promoting CTL-dependent clearance of lymphocytic choriomeningitis virus. Proceedings of the National Academy of Sciences of the United States of America, 2008. 105(2): p. 62934.

321. Turnbull, I.R., et al., Antibiotics improve survival in sepsis independent of injury severity but do not change mortality in mice with markedly elevated interleukin 6 levels. Shock, 2004. 21(2): p. 121-5.

322. Mclnnes, I.B. and G. Schett, Cytokines in the pathogenesis of rheumatoid arthritis. Nat Rev Immunol, 2007. 7(6): p. 429-442.

323. Fortunati, E., et al., Human neutrophils switch to an activated phenotype after homing to the lung irrespective of inflammatory disease. Clinical and Experimental Immunology, 2009. 155(3): p. 559-566. 
References.

324. van Oostrom, A.J., et al., Increased expression of activation markers on monocytes and neutrophils in type 2 diabetes. The Netherlands Journal of Medicine, 2004. 62(9): p. 3205.

325. Lee, W.L., R.E. Harrison, and S. Grinstein, Phagocytosis by neutrophils. Microbes Infect, 2003. 5(14): p. 1299-306.

326. Sahoo, M., et al., Neutrophil Elastase Causes Tissue Damage That Decreases Host Tolerance to Lung Infection with Burkholderia Species. PLoS Pathogens, 2014. 10(8): p. e1004327.

327. Bardoel, Bart W., et al., The Balancing Act of Neutrophils. Cell Host \& Microbe, 2014. 15(5): p. 526-536.

328. Jancinova, V., et al., Inhibition of FMLP-stimulated neutrophil chemiluminescence by blood platelets increased in the presence of the serotonin-liberating drug chloroquine. Thromb Res, 2003. 109(5-6): p. 293-8.

329. Losche, W., et al., Inhibition of leukocyte chemiluminescence by platelets: role of plateletbound fibrinogen. Platelets, 2001. 12(1): p. 15-9.

330. Deree, J., et al., Neutrophil degranulation and the effects of phosphodiesterase inhibition. Journal of Surgical Research, 2006. 133(1): p. 22-8.

331. Lacy, P., Mechanisms of Degranulation in Neutrophils. Allergy, Asthma, and Clinical Immunology: Official Journal of the Canadian Society of Allergy and Clinical Immunology, 2006. 2(3): p. 98-108.

332. Diacovo, T.G., et al., Neutrophil rolling, arrest, and transmigration across activated, surface-adherent platelets via sequential action of P-selectin and the beta 2-integrin CD11b/CD18. Blood, 1996. 88(1): p. 146-57.

333. Le Cabec, V., et al., Complement receptor 3 (CD11b/CD18) mediates type I and type II phagocytosis during nonopsonic and opsonic phagocytosis, respectively. Journal of Immunology, 2002. 169(4): p. 2003-9.

334. Belaaouaj, A., K.S. Kim, and S.D. Shapiro, Degradation of outer membrane protein A in Escherichia coli killing by neutrophil elastase. Science, 2000. 289(5482): p. 1185-8.

335. Kawabata, K., T. Hagio, and S. Matsuoka, The role of neutrophil elastase in acute lung injury. European Journal of Pharmacology, 2002. 451(1): p. 1-10.

336. Doring, G., The role of neutrophil elastase in chronic inflammation. American Journal of Respiratory and Critical Care Medicine, 1994. 150(6 Pt 2): p. S114-7.

337. Hammond, M.E., et al., IL-8 induces neutrophil chemotaxis predominantly via type I IL-8 receptors. Journal of Immunology, 1995. 155(3): p. 1428-33.

338. Dasu, M.R., A.C. Riosvelasco, and I. Jialal, Candesartan inhibits Toll-like receptor expression and activity both in vitro and in vivo. Atherosclerosis, 2009. 202(1): p. 76-83.

339. Larrayoz, I.M., et al., Candesartan reduces the innate immune response to lipopolysaccharide in human monocytes. Journal of Hypertension, 2009. 27(12): p. 236576.

340. Barakat, W., et al., Candesartan and glycyrrhizin ameliorate ischemic brain damage through downregulation of the TLR signaling cascade. European Journal of Pharmacology, 2014. 724: p. 43-50. 
References.

341. Land, W.G., The Role of Damage-Associated Molecular Patterns (DAMPs) in Human Diseases: Part II: DAMPs as diagnostics, prognostics and therapeutics in clinical medicine. Sultan Qaboos University Medical Journal, 2015. 15(2): p. e157-e170. 
Appendices.

Appendices.

Page / 263 
Appendices.

Appendix 1. Antibody information (in order of use).

\begin{tabular}{|c|c|c|c|c|}
\hline Antibody target & Fluorophore & Clone & Isotype (all mouse) & Manufacturer \\
\hline CD42a & PerCP & Beb1 & $\operatorname{lgG1,K}$ & BD Bioscience \\
\hline TLR9 & FITC & $26 C 593.2$ & $\lg G 1, \mathrm{~K}$ & Life Technologies \\
\hline CD64 & APC/Cy7 & 10.1 & $\lg G 1, \mathrm{~K}$ & BioLegend \\
\hline CD62p & $P E$ & AK-4 & $\lg G 1, \mathrm{~K}$ & BioLegend \\
\hline CD63 & AF647 & $\mathrm{H} 5 \mathrm{C} 6$ & $\lg G 1, \mathrm{~K}$ & BioLegend \\
\hline PAC1 & FITC & PAC1 & $\lg M, \mathrm{k}$ & BD Bioscience \\
\hline CD62p & BV421 & AK-4 & $\lg G 1, \mathrm{~K}$ & BD Bioscience \\
\hline CD42b & Biotin & AK2 & $\operatorname{lgG} 1, \mathrm{~K}$ & Life Technologies \\
\hline CD64 & BV421 & 10.1 & $\operatorname{lgG} 1, \mathrm{k}$ & BD Bioscience \\
\hline CD3 & BV510 & UCHT1 & $\operatorname{lgG} 1, \mathrm{k}$ & BD Bioscience \\
\hline CD4 & $\mathrm{APC} / \mathrm{H7}$ & RPA-T4 & $\operatorname{lgG} 1, \mathrm{k}$ & BD Bioscience \\
\hline CD8 & AF647 & RPA-T8 & $\lg G 1, \mathrm{k}$ & BD Bioscience \\
\hline CD38 & BB515 & HIT2 & $\lg G 1, \mathrm{~K}$ & BD Bioscience \\
\hline HLA-DR & PE/Cy7 & G46-6 & $\lg G 2 \mathrm{a}, \mathrm{k}$ & BD Bioscience \\
\hline CD16 & BV421 & $3 G 8$ & $\lg G 1, \mathrm{~K}$ & BD Bioscience \\
\hline CD66b & BB515 & G10F5 & $\lg M, \mathrm{~K}$ & BD Bioscience \\
\hline CD45 & PE/Cy7 & $\mathrm{HI} 30$ & $\lg G 1, \mathrm{~K}$ & BD Bioscience \\
\hline CD11b & BV510 & ICRF44 & $\lg G 1, \mathrm{~K}$ & BD Bioscience \\
\hline CD62L & APC & DREG-56 & $\lg G 1, \mathrm{~K}$ & BioLegend \\
\hline \multirow[t]{7}{*}{ Isotype controls } & PerCP & MOPC-21 & $\operatorname{lgG1,K}$ & BD Bioscience \\
\hline & FITC & MOPC-21 & $\lg G 1, \mathrm{k}$ & BioLegend \\
\hline & APC/Cy7 & MOPC-21 & $\operatorname{lgG} 1, \mathrm{k}$ & BioLegend \\
\hline & PE & MOPC-21 & $\operatorname{lgG} 1, \mathrm{k}$ & BioLegend \\
\hline & AF647 & MOPC-21 & $\operatorname{lgG} 1, \mathrm{k}$ & BioLegend \\
\hline & FITC & G155-228 & $\lg M, k$ & BD Bioscience \\
\hline & BV421 & $x 40$ & $\lg G 1, \mathrm{~K}$ & BD Bioscience \\
\hline
\end{tabular}




\begin{tabular}{|c|c|c|c|c|}
\hline Antibody target & Fluorophore & Clone & Isotype (all mouse) & Manufacturer \\
\hline \multirow[t]{6}{*}{ Isotype controls } & BV510 & $x 40$ & $\lg G 1, \mathrm{~K}$ & BD Bioscience \\
\hline & BB515 & $x 40$ & $\lg G 1, \mathrm{~K}$ & BD Bioscience \\
\hline & $\mathrm{PE} / \mathrm{Cy} 7$ & G155-178 & $\lg G 2 a, k$ & BD Bioscience \\
\hline & APC & MOPC-21 & $\lg G 1, \mathrm{k}$ & BD Bioscience \\
\hline & BB515 & G20-127 & $\lg M, k$ & BD Bioscience \\
\hline & PE/Cy7 & MOPC-21 & $\lg G 1, \mathrm{~K}$ & BD Bioscience \\
\hline
\end{tabular}


Appendices.

Appendix 2. Solutions.

\section{General solutions.}

10X Phosphate-buffered saline (PBS), (w/v), pH 7.4.

\begin{tabular}{|l|l|r|}
\hline & Concentration (mM) & $\mathrm{g} / \mathrm{L}$ \\
\hline $\mathrm{NaCl}$ & 1370.00 & 80.0 \\
\hline $\mathrm{KCl}$ & 26.83 & 2.0 \\
\hline $\mathrm{KH}_{2} \mathrm{PO}_{4}$ & 17.64 & 2.4 \\
\hline $\mathrm{Na}_{2} \mathrm{HPO}_{4}$ & 101.44 & 14.4 \\
\hline
\end{tabular}

Flow cytometry solutions.

$1 x$ staining buffer $(v / v), p H 7.4$

\begin{tabular}{|l|l|r|}
\hline & Concentration (\%) & $\mathrm{mL} / \mathrm{L}$ \\
\hline Fetal calf serum (FCS) & $2.0 \%$ & 20 \\
\hline Sodium azide & $0.1 \%$ & 1 \\
\hline PBS & $97.9 \%$ & 979 \\
\hline
\end{tabular}




\section{Western blot solutions.}

1x radio-immunoprecipitation buffer, $p H 7.4$

\begin{tabular}{|l|l|r|}
\hline & Concentration & $\mathrm{g} / \mathrm{L}, \mathrm{mL} / \mathrm{L}$ \\
\hline Tris- $\mathrm{HCl}$ & $10 \mathrm{mM}$ & $1.20 \mathrm{~g}$ \\
\hline $\mathrm{NaCl}$ & $150 \mathrm{mM}$ & $8.78 \mathrm{~g}$ \\
\hline EDTA & $1 \mathrm{mM}$ & $0.37 \mathrm{~g}$ \\
\hline SDS & $0.1 \%$ & $1.00 \mathrm{~g}$ \\
\hline Sodium deoxycholate & $1.0 \%$ & $10.00 \mathrm{~g}$ \\
\hline Triton-X-100 & $1.0 \%$ & $10.00 \mathrm{~mL}$ \\
\hline
\end{tabular}

Laemmli buffer.

\begin{tabular}{|l|l|r|}
\hline & Concentration & $\mathrm{g} / \mathrm{L}, \mathrm{mL} / \mathrm{L}$ \\
\hline $1.5 \mathrm{M}$ Tris- $\mathrm{HCl}, \mathrm{pH} 6.8$ & $30 \mathrm{mM}$ & $20 \mathrm{~mL}$ \\
\hline SDS & $2 \%$ & $20 \mathrm{~g}$ \\
\hline $80 \%$ glycerol & $20 \%$ & $250 . \mathrm{mL}$ \\
\hline Bromophenol blue & $1 \%$ & $10 \mathrm{~g}$ \\
\hline
\end{tabular}

SDS-PAGE running buffer.

\begin{tabular}{|l|l|r|}
\hline & Concentration & g/L \\
\hline Tris & $25 \mathrm{mM}$ & 3.03 \\
\hline Glycine & $192 \mathrm{mM}$ & 14.41 \\
\hline SDS & $0.1 \%$ & 1.00 \\
\hline
\end{tabular}


Western blot transfer buffer.

\begin{tabular}{|l|l|r|}
\hline & Concentration & $\mathrm{g} / \mathrm{L}, \mathrm{mL} / \mathrm{L}$ \\
\hline Tris & $25 \mathrm{mM}$ & $3.03 \mathrm{~g}$ \\
\hline Glycine & $192 \mathrm{mM}$ & $14.41 \mathrm{~g}$ \\
\hline Methanol & $10 \%$ & $100 \mathrm{~mL}$ \\
\hline
\end{tabular}

1x Tris-buffered saline (TBS), pH 7.4.

\begin{tabular}{|l|l|r|}
\hline & & $\mathrm{g} / \mathrm{L}$ \\
\hline Tris & $50 \mathrm{mM}$ & 6.06 \\
\hline $\mathrm{NaCl}$ & $150 \mathrm{~mL}$ & 8.78 \\
\hline
\end{tabular}

1x TBS-Tween 20, pH7.4.

1x TBS containing $0.1 \%(1 \mathrm{~mL} / \mathrm{L})$ Tween-20.

\section{SDS-PAGE gels.}

8\% separating gel (2 gels, $1.5 \mathrm{~mm}$ thickness).

\begin{tabular}{|l|r|}
\hline & $\mathrm{mL}$ \\
\hline $\mathrm{dd} \mathrm{H}_{2} \mathrm{O}$ & 7.400 \\
\hline $1.5 \mathrm{M}$ Tris-HCl, pH6.8 & 4.000 \\
\hline $10 \%$ SDS & 0.160 \\
\hline $10 \%$ APS & 0.160 \\
\hline TEMED & 0.016 \\
\hline Acrylamide & 4.270 \\
\hline
\end{tabular}


4\% stacking gel (4 gels, $1.5 \mathrm{~mm}$ thickness)

\begin{tabular}{|l|r|}
\hline & $\mathrm{mL}$ \\
\hline $\mathrm{dd} \mathrm{H}_{2} \mathrm{O}$ & 6.10 \\
\hline $1.5 \mathrm{M}$ Tris-HCl, $\mathrm{pH} 6.8$ & 2.50 \\
\hline $10 \%$ SDS & 0.10 \\
\hline $10 \%$ APS & 0.05 \\
\hline TEMED & 0.01 \\
\hline Acrylamide & 1.33 \\
\hline
\end{tabular}

\section{Cell culture solutions.}

Cell culture media $(v / v)$, filter-sterilized using $0.22 \mu \mathrm{M}$ syringe filter.

\begin{tabular}{|l|l|r|}
\hline & Concentration $(\%)$ & $\mathrm{mL} / \mathrm{L}$ \\
\hline Dulbecco's Modified Eagle Medium & 85.9 & 859 \\
\hline FCS & 10.0 & 100 \\
\hline L-glutamate $(200 \mathrm{mM})$ & 1.0 & 10 \\
\hline Penicillin $(100 \mathrm{U} / \mathrm{mL}) /$ Streptomycin $(10 \mathrm{mg} / \mathrm{mL})$ & 1.0 & 10 \\
\hline HEPESs buffer $(1 \mathrm{M})$ & 1.0 & 1 \\
\hline B-mercaptoethanol & 0.1 & 10 \\
\hline Non-essential amino acids $(10 \mathrm{nM})$ & 1.0 & 10 \\
\hline
\end{tabular}

Isolation buffer ( $/ \mathrm{v})$.

\begin{tabular}{|l|l|r|}
\hline & Concentration & $\mathrm{mL} / \mathrm{L}$ \\
\hline PBS & $97.8 \%$ & 986 \\
\hline BSA $(10 \%)$ & $0.1 \%$ & 10 \\
\hline EDTA $(0.5 \mathrm{M})$ & $2 \mathrm{mM}$ & 4 \\
\hline
\end{tabular}

